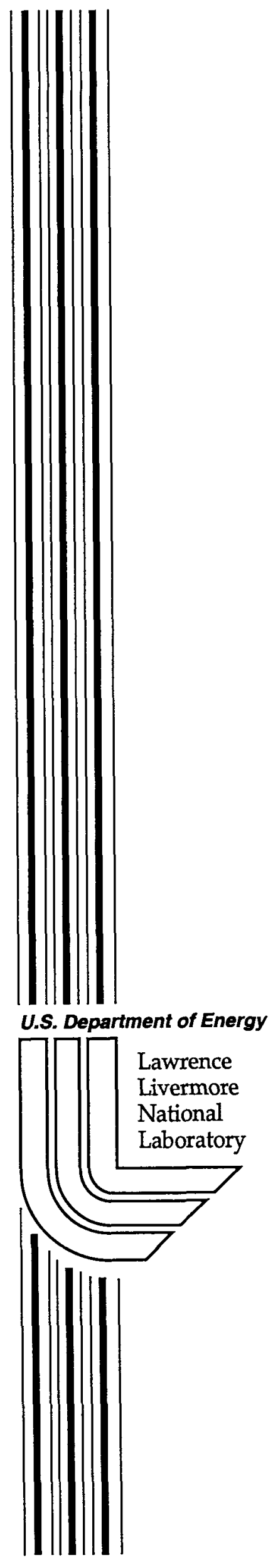

UCRL-LR-146433

Thesis

\title{
Optical Parametric Amplification for High Peak and Average Power
}

\author{
I. Jovanovic \\ Ph.D
}

November 26, 2001 


\section{DISCLAIMER}

This document was prepared as an account of work sponsored by an agency of the United States Government. Neither the United States Government nor the University of California nor any of their employees, makes any warranty, express or implied, or assumes any legal liability or responsibility for the accuracy, completeness, or usefulness of any information, apparatus, product, or process disclosed, or represents that its use would not infringe privately owned rights. Reference herein to any specific commercial product, process, or service by trade name, trademark, manufacturer, or otherwise, does not necessarily constitute or imply its endorsement, recommendation, or favoring by the United States Government or the University of California. The views and opinions of authors expressed herein do not necessarily state or reflect those of the United States Government or the University of California, and shall not be used for advertising or product endorsement purposes.

This work was performed under the auspices of the U.S. Department of Energy by the University of California, Lawrence Livermore National Laboratory under Contract No. W-7405-Eng-48.

This report has been reproduced directly from the best available copy.

Available to DOE and DOE contractors from the Office of Scientific and Technical Information P.O. Box 62, Oak Ridge, TN 37831

Prices available from (423) 576-8401 http://apollo.osti.gov/bridge/

Available to the public from the National Technical Information Service

U.S. Department of Commerce 5285 Port Royal Rd., Springfield, VA 22161 http://www.ntis.gov/

$\mathrm{OR}$

Lawrence Livermore National Laboratory Technical Information Department's Digital Library http://www.llnl.gov/tid/Library.html 
Igor Jovanovic: “Optical Parametric Amplification for

High Peak and Average Power"

Dissertation, UC Berkeley

Filing date: December 10, 2001

The following records of invention have been filed related to the material contained in this dissertation:

1. I. Jovanovic and R. A. Bonner, "High Gain Preamplifier Based on Optical Parametric Amplification," IL-10835 (Feb 26, 2001)

2. I. Jovanovic and B. J. Comaskey, "High Average Power Scaling of Optical Parametric Amplification Through Cascaded Difference Frequency Generators," IL-10885 (Jun 7, 2001)

3. I. Jovanovic and C. P. J. Barty, "A Hybrid Optical Parametric - Ti:sapphire Chirped Pulse Amplification System," IL-10909 (Aug 1, 2001)

4. I. Jovanovic and C. P. J. Barty, "A Method for Coherent White Light Amplification," IL-10933 (Sep 13, 2001)

5. I. Jovanovic and C. A. Ebbers, "Nondegenerate optical parametric chirped pulse amplifier," (Nov 6, 2001)

The following LDRD has been used to fund this research:

'New Front End Technology", 00-ERD-070. 
Optical Parametric Amplification for

High Peak and Average Power

by

Igor Jovanovic

B.S. (University of Zagreb) 1997

A dissertation submitted in partial satisfaction of the requirements for the degree of

Doctor of Philosophy in

Engineering - Nuclear Engineering

in the

GRADUATE DIVISION

of the

UNIVERSITY OF CALIFORNIA, BERKELEY

Committee in charge:

Professor Edward C. Morse, Chair

Professor Jasmina L. Vujic

Professor Dmitry Budker

Fall 2001 
The dissertation of Igor Jovanovic is approved:

\begin{tabular}{ll}
\hline Chair & Date \\
& \\
\hline & Date \\
\hline & Date
\end{tabular}

University of California, Berkeley

Fall 2001 
Optical Parametric Amplification for High Peak and Average Power

Copyright 2001

by

Igor Jovanovic 


\author{
Abstract \\ Optical Parametric Amplification for High Peak and Average Power \\ by \\ Igor Jovanovic \\ Doctor of Philosophy in Engineering - Nuclear Engineering \\ University of California, Berkeley \\ Professor Edward C. Morse, Chair
}

Optical parametric amplification is an established broadband amplification technology based on a second-order nonlinear process of difference-frequency generation (DFG). When used in chirped pulse amplification (CPA), the technology has been termed optical parametric chirped pulse amplification (OPCPA). OPCPA holds a potential for producing unprecedented levels of peak and average power in optical pulses through its scalable ultrashort pulse amplification capability and the absence of quantum defect, respectively.

The theory of three-wave parametric interactions is presented, followed by a description of the numerical model developed for nanosecond pulses. Spectral, temperature and angular characteristics of OPCPA are calculated, with an estimate of pulse contrast. An OPCPA system centered at $1054 \mathrm{~nm}$, based on a commercial tabletop Q-switched pump laser, was developed as the front end for a large Nd:glass petawattclass short-pulse laser. The system does not utilize electro-optic modulators or multi-pass amplification. The obtained overall $6 \%$ efficiency is the highest to date in OPCPA that uses a tabletop commercial pump laser. The first compression of pulses amplified in 
highly nondegenerate OPCPA is reported, with the obtained pulse width of $60 \mathrm{fs}$. This represents the shortest pulse to date produced in OPCPA. Optical parametric amplification in $\beta$-barium borate was combined with laser amplification in Ti:sapphire to produce the first hybrid CPA system, with an overall conversion efficiency of $15 \%$. Hybrid CPA combines the benefits of high gain in OPCPA with high conversion efficiency in Ti:sapphire to allow significant simplification of future tabletop multiterawatt sources. Preliminary modeling of average power limits in OPCPA and pump laser design are presented, and an approach based on cascaded DFG is proposed to increase the average power beyond the single-crystal limit. Angular and beam quality effects in optical parametric amplification are modeled using plane-wave decomposition. Finally, some possible future applications are outlined.

It is expected that OPCPA will be an important technology for future high power short-pulse lasers and will allow novel applications in high-field science. Its scalability beyond 10 petawatts using the existing diffractive grating compression technology, and the possibility of producing average powers beyond $1 \mathrm{~kW}$ in ultrashort pulses provide motivation for future work. 


\section{Table of Contents}

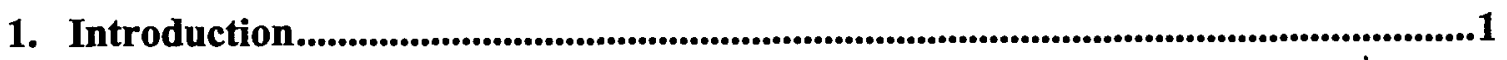

2. Nanosecond three-wave optical parametric interactions .........................................5

2.1. Derivation of the wave equation .....................................................................6

2.2. Coupled wave equations for difference-frequency generation .............................10

2.3. Solutions of wave equations for optical parametric amplification with nanosecond pulses...................................................................................13

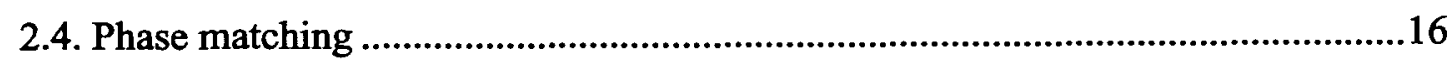

2.5. Light waves on the boundary of vacuum and nonlinear medium ........................22

3. Modeling of optical parametric chirped pulse amplification...................................26

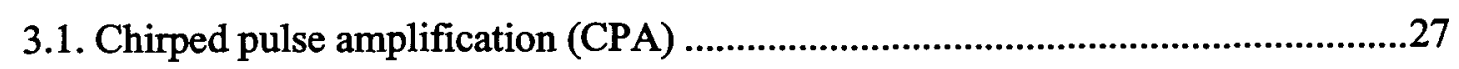

3.2. Optical parametric chirped pulse amplification (OPCPA) .................................29

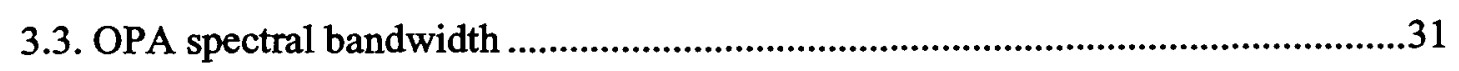

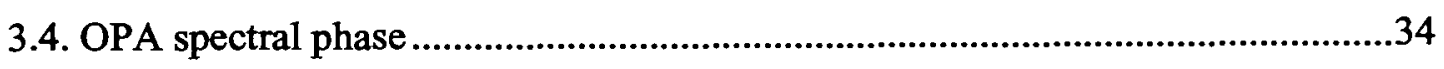

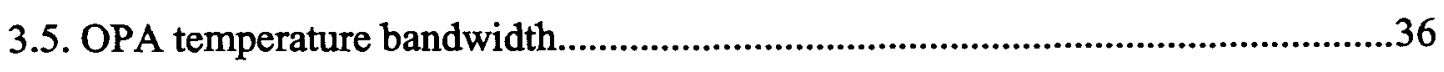

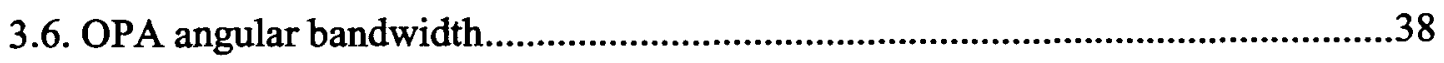

3.7. Pulse contrast in optical parametric amplification..............................................4

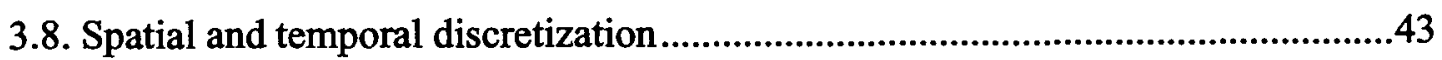

4. Optical parametric amplifier as a preamplifier for high-energy

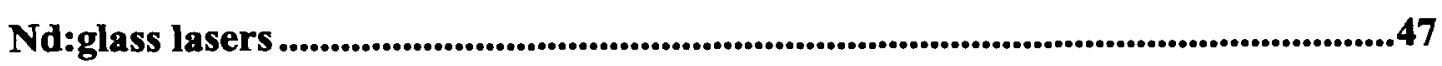

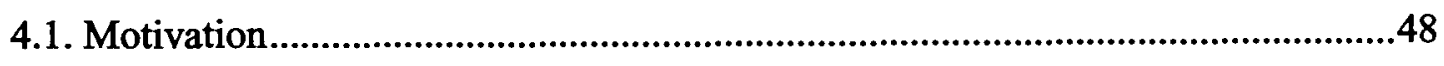

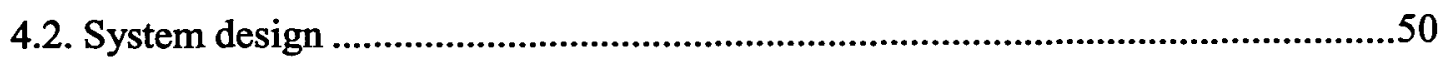

4.3. Experimental results - preamplifier............................................................57

4.4. Experimental results - power amplifier and compression .................................60

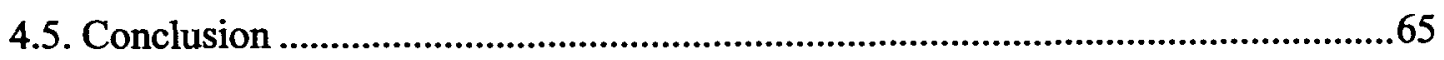

5. Nondegenerate optical parametric chirped pulse amplification ............................68

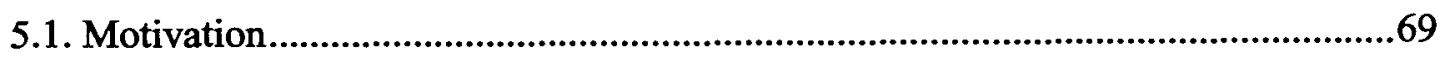

5.2. Numerical modeling of simple ultrashort pulse OPCPA ....................................71

5.3. Angular dispersion and temporal modulation for ultrabroad

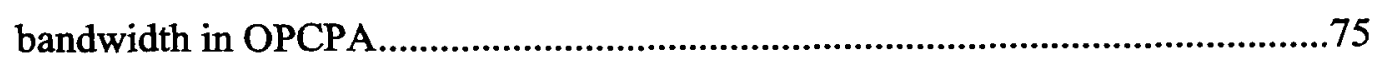




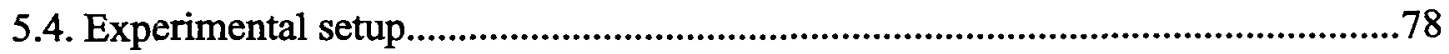

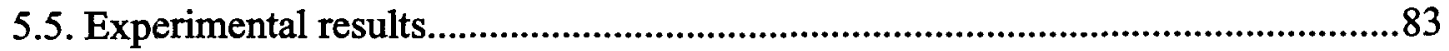

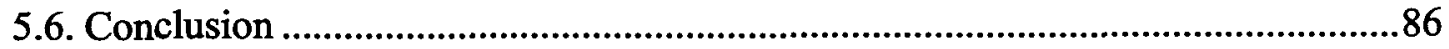

6. Hybrid chirped pulse amplification for multi-TW tabletop sources.......................88

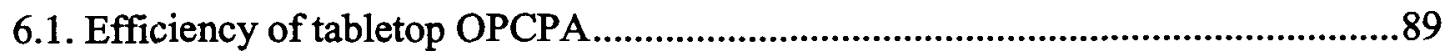

6.2. The hybrid optical parametric-laser CPA system .............................................91

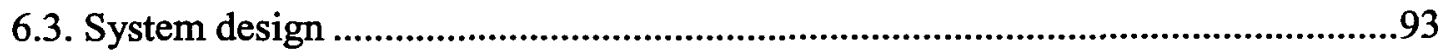

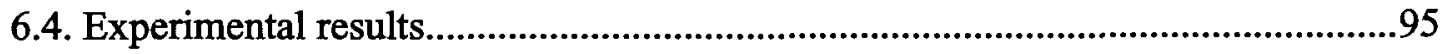

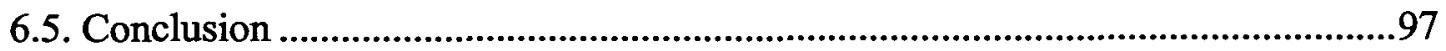

7. High average power optical parametric chirped pulse amplification.....................99

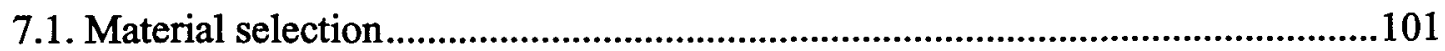

7.2. Modeling of average power limits ..................................................................103

7.3. Cascaded difference frequency generation for high average power ...................108

7.4. High average power pump laser design ...........................................................110

7.5. High repetition rate seeder laser ................................................................115

8. Angular effects and beam quality in optical parametric amplification ................117

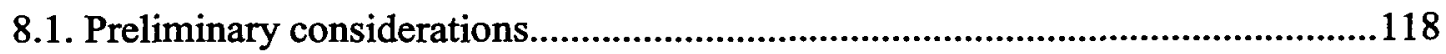

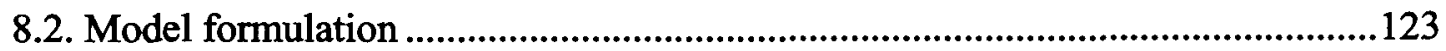

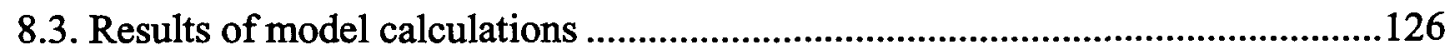

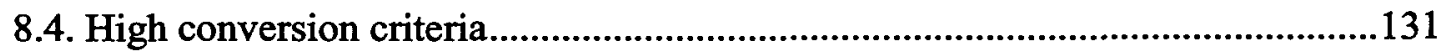

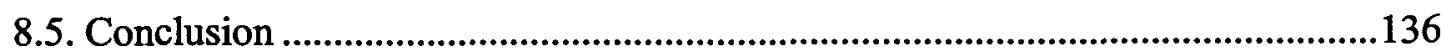

9. Applications of optical parametric chirped pulse amplification ...........................138

9.1. Fast ignitor for inertial confinement fusion .........................................................138

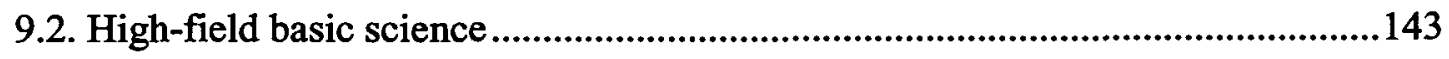

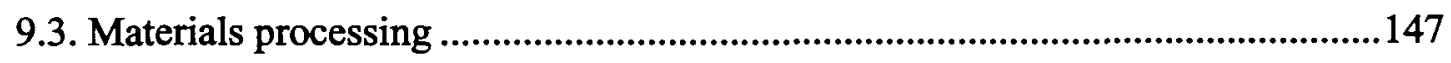

9.4. Broadband amplification for telecommunications............................................148

10. Conclusion ..................................................................................................................................150

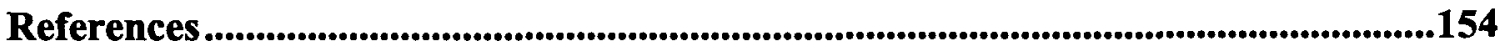




\section{List of Figures}

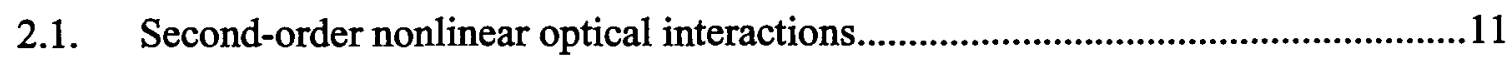

2.2. Qualitative behavior of the solutions for signal and idler in OPA operating in the small signal regime .14

2.3. Qualitative behavior of the solutions for signal and idler in OPA operating in the regime of depleted pump.......................................................16

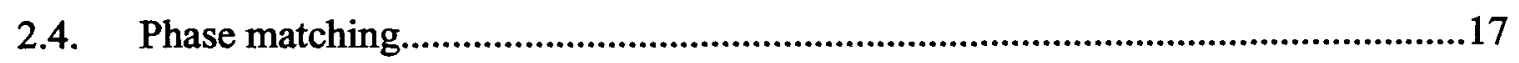

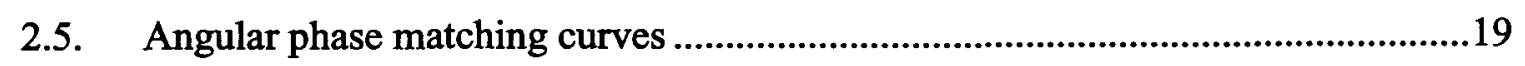

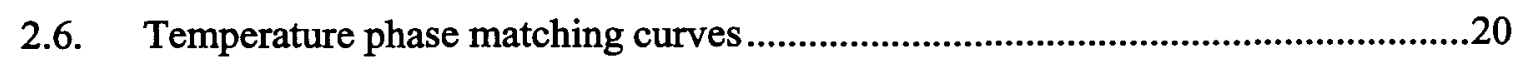

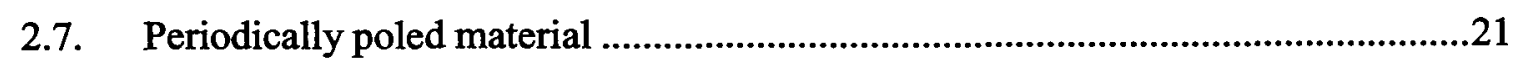

2.8. Poling period in lithium niobate as a function of signal wavelength .....................21

2.9. Waves on the boundary of vacuum (air) and nonlinear medium ..........................23

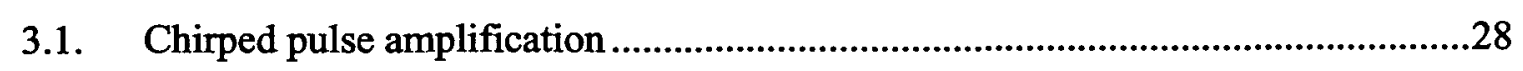

3.2. Optical parametric chirped pulse amplification ......................................................30

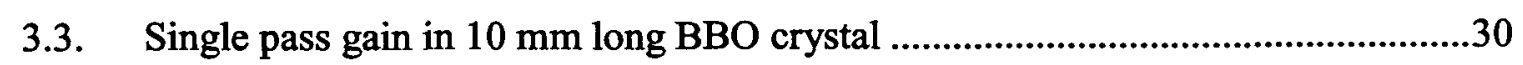

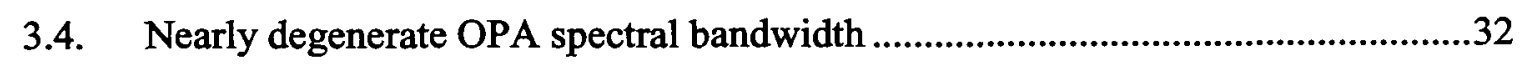

3.5. Nondegenerate OPA spectral bandwidth for..........................................................33

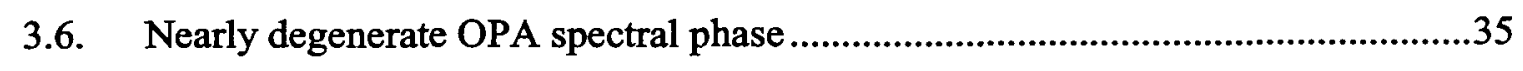

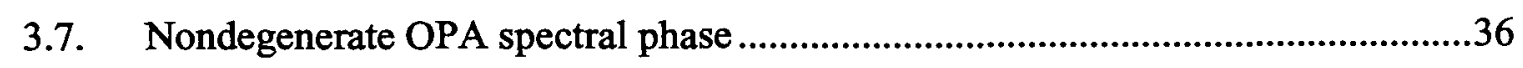

3.8. Nearly degenerate OPA temperature bandwidth.....................................................37

3.9. Nondegenerate OPA temperature bandwidth...........................................................38

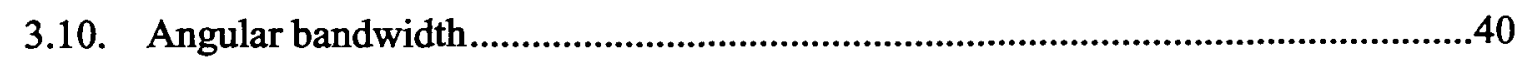

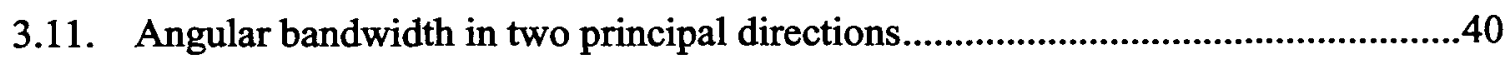

3.12. Transverse interaction of signal and pump in the numerical model ......................46

4.1. Design of the Petawatt laser front end ..................................................................48

4.2. Experimental OPCPA setup for replacing Ti:sapphire regenerative amplifiers ....51

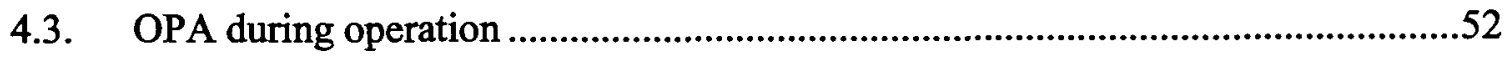

4.4. Pump beam near intensity profile ...................................................................52

4.5. Spatio-temporal evolution of the pump pulse originating in an unstable resonator (Spectra-Physics GCR 270-10). 


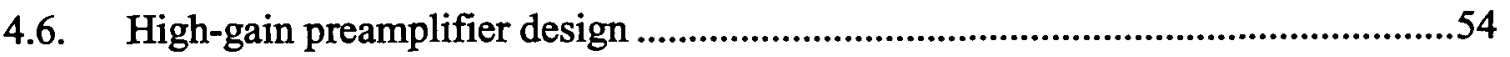

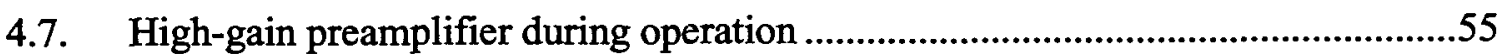

4.8. Experimental and theoretical results for single pass gain from

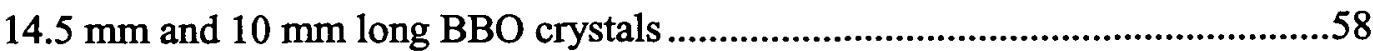

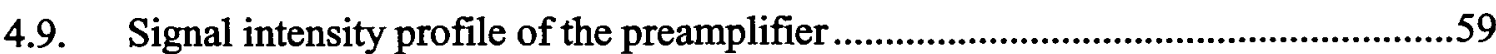

4.10. Experimental extraction data from the final $\mathrm{BBO}$ amplifier................................61

4.11. Seed and amplified signal spectra from preamplifier and power amplifier...........62

4.12. Instantaneous recorded pump power after OPCPA …..........................................63

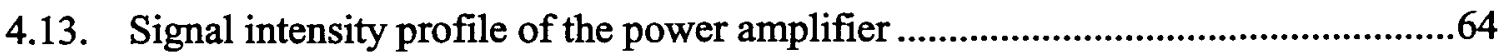

4.14. Recompressed pulse intensity autocorrelation in nearly degenerate OPCPA........65

5.1. Ultrashort pulse amplification in two BBO crystals in the small signal regime....73

5.2. Ultrashort pulse amplification in one BBO crystal in the regime of depleted pump

5.3. Intensity spectrum and noncollinear angle required to achieve perfect wave vector matching for a $10 \mathrm{fs}$ pulse

5.4. A small spectral shift occurs in amplified signal due to wavelength-dependent gain in OPA .78

5.5. Experimental nondegenerate setup for ultrashort pulse amplification....................79

5.6. Interferometric autocorrelation of the oscillator pulse..........................................79

5.7. Near-field transverse intensity profile of the pump pulse ...................................80

5.8. Spatio-temporal evolution of the pump pulse in Spectra-Physics GCR Pro 350-10 laser....................................................................81

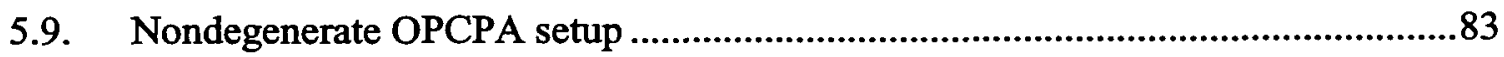

5.10. Spectral profiles of the seed and amplified signal at two energy levels ................ 84

5.11. Transverse intensity profiles for signal in nondegenerate OPCPA........................84

5.12. Recompressed pulse intensity autocorrelation in nondegenerate OPCPA.............86

5.12. Calculated recompressed pulse shape in nondegenerate OPCPA..........................86

6.1. The concept of hybrid chirped pulse amplification...............................................92

6.2. Experimental setup for demonstration of hybrid chirped pulse amplification.......94

6.3. Beam profile of the amplified pulse in hybrid CPA ...........................................96

6.4. Oscillator and amplified signal spectra in hybrid CPA......................................96 
6.5. Experimental setup of the hybrid CPA experiment ........................................97

7.1. Optical parametric amplification as a perfectly elastic process ............................100

7.2. Coupled iterative nonlinear conversion - thermal diffusion method.....................104

7.3. Signal pulse energy as a function of repetition rate in

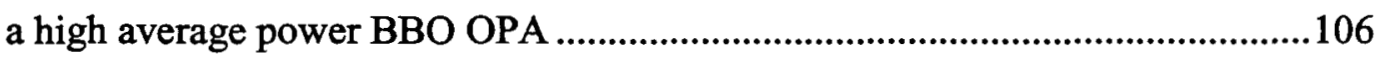

7.4. Calculation results for high average power OPCPA operating at $5 \mathrm{kHz}$.............107

7.5. Final OPA crystal in a high average power system ..........................................109

7.6. Distribution of heat load in OPA through cascaded difference frequency generation .....................................................110

7.7. Design of an $80 \mathrm{~W}, 532 \mathrm{~nm}$ pump laser for high average power OPCPA...........112

7.8. Design of the 2-rod double-pass amplifier......................................................113

7.9. Single amplifier depolarization and thermal lens measurements..........................114

7.10. Seeder laser design for high average power OPCPA pump laser .......................115

7.11. High repetition rate seeder laser......................................................................116

8.1. Normalized intensity distributions in divergence angles for a gaussian and a top-hat beam .............................................................121

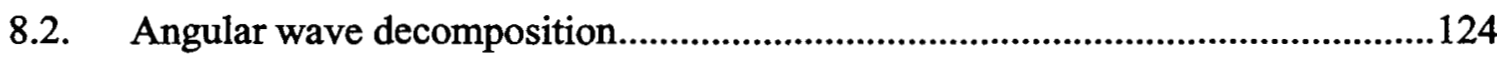

8.3. Small signal gain in BBO for different divergence parameters ...........................126

8.4. Small signal gain in KDP for different divergence parameters ...........................127

8.5. Small signal gain in NCPM LBO for different divergence parameters ...............127

8.6. Large signal gain in $\mathrm{BBO}$ for different divergence parameters ...........................129

8.7. Large signal gain in KDP for different divergence parameters ...........................129

8.8. Large signal gain in NCPM LBO for different divergence parameters ................130

8.9. Maximum conversion in $\mathrm{BBO}$ as a function of the ratio of seed and pump input

9.1. A conceptual design for the NIF fast ignitor laser based on OPCPA front end

9.2. Temporal (spectral) shape of the signal in the

OPCPA front end of a fast ignitor laser

9.3. Conceptual design of a degenerate and nondegenerate

15 PW/100 PW all-OPCPA-based source 


\section{List of Tables}

4.1. Beam quality from the preamplifier in nearly degenerate OPCPA......................60

4.2. Beam quality from the power amplfier in nearly degenerate OPCPA...................64

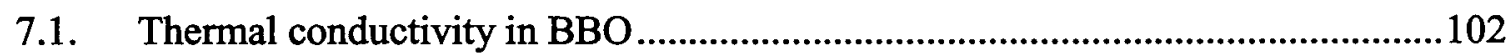

9.1. OPA design parameters for the OPCPA-based

front end of the fast ignitor laser..... 


\section{Chapter 1}

\section{Introduction}

Investigation of nonlinear parametric processes has been at the forefront of optics research as the light intensities reached the level necessary for nonlinear effects to be readily observed. ${ }^{1}$ Optical parametric amplification ${ }^{2}$ and oscillation ${ }^{3}$ through differencefrequency generation (DFG) are some of the earliest observed effects in nonlinear optics, and the application of optical parametric amplifiers (OPAs) for light amplification is practiced extensively. Today, optical parametric oscillators (OPOs) offer a way to produce widely tunable coherent light from solid-state gain media for frequency-sensitive applications such as spectroscopy. ${ }^{4}$

The development of nonlinear optics benefited greatly from powerful laser pulse generation through Q-switching ${ }^{5}$ and mode locking. ${ }^{6}$ More recently, chirped pulse amplification (CPA) technology ${ }^{7}$ allowed the scaling of peak power beyond 1 petawatt $^{8}$ and greater than $10^{21} \mathrm{~W} / \mathrm{cm}^{2}$ peak focused intensity. ${ }^{9}$ The development of CPA allows explorations of extreme nonlinear optics such as high harmonic generation (HHG). ${ }^{10}$ In addition, progress in high average power laser amplification allows the development of high average power nonlinear devices. This benefits applications requiring high repetition rates, such as short-pulse materials processing. ${ }^{11}$ 
Historically, the development of nonlinear optical devices has been primarily driven by the evolving laser technology. As the CPA technology approaches its limits in pulse energy and pulse width, an opportunity exists for the first time to use parametric devices to allow scaling of peak and average power of optical pulses beyond the limits imposed by the characteristics of laser materials. Extremely broad spectral bandwidth, minimal heat deposition and aperture scalability represent a combination of key characteristics of optical parametric amplification not present in any solid-state laser gain material simultaneously. Used for amplification in CPA, optical parametric amplification has been termed parametric chirped pulse amplification (PCPA) ${ }^{12}$ and, more recently, optical parametric chirped pulse amplification (OPCPA). ${ }^{13}$

High peak and average power scaling is not the only motivation behind the use of OPCPA. Additional advantages in performance of small-scale OPCPA exist when compared to conventional laser CPA. These include pulse contrast, simplicity and cost. Finally, an opportunity exists to use OPCPA in combination with conventional laser CPA, producing more efficient tabletop short-pulse sources than the ones accessible with either approach alone.

The purpose of this dissertation is to offer a numerical analysis and small-scale experimental demonstration of certain aspects of OPCPA relevant for producing high peak power and high average power pulses. This includes the development of actual devices, which can replace entire high-power lasers, or some of their components.

Following this introduction, a formal mathematical description of nanosecond three-wave parametric interactions is presented, with a special emphasis on DFG and OPA. Several key concepts in OPA are discussed in more detail, such as the phase 
matching techniques and vacuum-nonlinear material boundaries. In Chapter 3, a more thorough background on CPA and OPCPA is given, which is accompanied by a description of a numerical model for nanosecond OPCPA devices. The described numerical model can assess the performance of OPCPA pumped by a variety of pump lasers. A detailed calculation of spectral, temperature and angular bandwidths is given to establish some important limitations of light amplification in OPCPA.

Chapter 4 describes a practical demonstration of a replacement for a $1 \mu \mathrm{m}$ broadband regenerative amplifier CPA with an OPCPA system. Intended as the front end for a high-power glass laser, OPCPA produced a 50-fold increase in pulse energy and a 10-fold improvement in conversion efficiency compared to previous experiments that used commercial Q-switched pump lasers. Successful pulse recompression and good amplified beam quality are encouraging the use of OPCPA in the front end of future short-pulse glass lasers.

A critical element of the scalability of OPCPA to high peak power is achieving ultrashort recompressed pulse width. This is demonstrated for the first time in a nondegenerate noncollinear OPCPA described in Chapter 5. Recompressed pulse width of $60 \mathrm{fs}$ and good energy extraction in the signal pulse temporal window agree well with the theoretical predictions for ultrashort pulse amplification in OPCPA. Overall extraction efficiency is dramatically improved by construction of the novel hybrid optical parametric-Ti:sapphire CPA system (Chapter 6). Highly efficient production of energetic $820-\mathrm{nm}$ pulses was achieved for the first time using a single commercial pump laser and no electro-optic modulators. It is expected that this approach will find use in both scientific and commercial applications. 
The development of high average power OPCPA depends greatly on construction of suitable nanosecond high average power pump lasers. Chapter 7 is dedicated to two important issues that have to be examined: average power limits and pump laser technology. First, realistic average power limits are determined for OPCPA based on the available absorption data. A proposal is formulated for higher power scaling through advanced cascaded architecture. Second, a possible pump laser technology is discussed along with preliminary experimental results.

High peak power and average power OPCPA will require high-power pump lasers. Since the laser beam quality typically degrades with the laser power, the influence of beam quality on conversion efficiency in OPCPA is of great concern. A numerical model presented in Chapter 8 tackles this problem by plane-wave decomposition of real laser beams. It establishes real requirements of the beam divergence that permit high conversion efficiency in OPA devices. High conversion criteria are also formulated for quick estimates of OPCPA performance. In the final two chapters, some key envisioned applications of OPCPA are addressed in more detail and a conclusion is given. 


\section{Chapter 2}

\section{Nanosecond three-wave}

\section{optical parametric interactions}

In the following text, an introduction to the phenomenology of parametric threewave interactions is given, with a focus on difference-frequency generation (DFG) in the nanosecond pulse regime. The use of nanosecond pulses allows the treatment to be significantly simplified when compared to the formalism that describes parametric interactions of ultrashort (femtosecond and picosecond) pulses.

First, the wave equation for an electromagnetic wave is derived from Maxwell's equations, and its convenient form is given in the frequency domain. Nonlinear polarization is introduced, characteristic for a nonlinear medium in strong laser field. Next, coupled wave equations are derived for DFG, and the simplification to plane waves is introduced. This is followed by analytical solutions of coupled wave equations in the regime of small signal gain and in the regime of depleted pump.

The importance of phase matching in parametric processes is described in more detail in section 4 . The most popular phase matching techniques are described in more detail, together with representative examples for some common nonlinear crystals. Finally, a more thorough description is presented for the nature of light wave propagation 
on a boundary between vacuum and nonlinear medium, followed by a derivation of angular wave vector mismatch.

While a rigorous approach to three-wave optical parametric interactions includes quantum mechanics, ${ }^{14}$ it is often sufficient and more illustrative to use the classical or the semiclassical picture. In the following text, the classical approach is taken following the combined work of Shen ${ }^{14}$ and Boyd. ${ }^{15}$ In the derivations and results presented in this Chapter and in the remainder of this dissertation, SI units are consistently used to avoid ambiguity.

\subsection{Derivation of the wave equation}

The propagation of an electromagnetic wave at a point in space and time $(\mathbf{r}, t)$ is described by Maxwell's equations:

$$
\begin{gathered}
\nabla \cdot \mathbf{D}(\mathbf{r}, t)=\rho(\mathbf{r}, t), \\
\nabla \cdot \mathbf{B}(\mathbf{r}, t)=0, \\
\nabla \times \mathbf{E}(\mathbf{r}, t)=-\frac{\partial \mathbf{B}(\mathbf{r}, t)}{\partial t}, \\
\nabla \times \mathbf{H}(\mathbf{r}, t)=\mathbf{J}(\mathbf{r}, t)+\frac{\partial \mathbf{D}(\mathbf{r}, t)}{\partial t},
\end{gathered}
$$

where $\mathbf{D}$ is the electric displacement, $\mathbf{E}$ is the electric field, $\mathbf{B}$ is the magnetic induction, $\mathbf{H}$ is the magnetic field, $\rho$ is the charge density, and $\mathbf{J}$ is the current density. The relationship between vector pairs $(\mathbf{D}, \mathbf{E})$ and $(\mathbf{B}, \mathbf{H})$ is determined by constitutive relations:

$$
\mathbf{D}(\mathbf{r}, t)=\varepsilon_{0} \mathbf{E}(\mathbf{r}, t)+\mathbf{P}(\mathbf{r}, t),
$$




$$
\mathbf{B}(\mathbf{r}, t)=\mu \mathbf{H}(\mathbf{r}, t) .
$$

In the constitutive relations, $\mathbf{P}$ is the dielectric polarization vector, $\varepsilon_{0}$ is the dielectric constant of vacuum, and $\mu$ is the magnetic permeability. In this derivation, only nonmagnetic materials $\left(\mu=\mu_{0}\right)$ are considered. Generally, the polarization vector can be expanded in its linear and nonlinear terms:

$$
\mathbf{P}(\mathbf{r}, t)=\sum_{i=1}^{\infty} \mathbf{P}^{(i)}(\mathbf{r}, t)=\mathbf{P}^{(1)}(\mathbf{r}, t)+\mathbf{P}^{\mathrm{NL}}(\mathbf{r}, t),
$$

where the linear term is

$$
\mathbf{P}^{(1)}(\mathbf{r}, t)=\varepsilon_{0} \int_{-\infty}^{\infty} \chi^{(1)}\left(\mathbf{r}-\mathbf{r}^{\prime}, t-\tau\right) \cdot \mathbf{E}(\mathbf{r}, \tau) \mathrm{d} \mathbf{r}^{\prime} \mathrm{d} \tau
$$

In this relation, $\chi^{(1)}$ is the linear susceptibility tensor. The second and third nonlinear terms are somewhat more complicated:

$$
\begin{aligned}
& \mathbf{P}^{(2)}(\mathbf{r}, t)=\int_{-\infty}^{\infty} \chi^{(2)}\left(\mathbf{r}-\mathbf{r}_{1}, t-\tau_{1} ; \mathbf{r}-\mathbf{r}_{2}, t-\tau_{2}\right): \mathbf{E}\left(\mathbf{r}, \tau_{1}\right) \mathbf{E}\left(\mathbf{r}, \tau_{2}\right) d \mathbf{r}_{1} d \tau_{1} d \mathbf{r}_{2} d \tau_{2}, \\
& \mathbf{P}^{(3)}(\mathbf{r}, t)=\int_{-\infty}^{\infty} \chi^{(3)}\left(\mathbf{r}-\mathbf{r}_{1}, t-\tau_{1} ; \mathbf{r}-\mathbf{r}_{2}, t-\tau_{2} ; \mathbf{r}-\mathbf{r}_{3}, t-\tau_{3}\right) \vdots \mathbf{E}\left(\mathbf{r}, \tau_{1}\right) \mathbf{E}\left(\mathbf{r}, \tau_{2}\right) \mathbf{E}\left(\mathbf{r}, \tau_{3}\right) \times \\
& d \mathbf{r}_{1} d \tau_{1} d \mathbf{r}_{2} d \tau_{2} d \mathbf{r}_{3} d \tau_{3},
\end{aligned}
$$

where $\chi^{(2)}$ and $\chi^{(3)}$ are the second and third order susceptibility tensors, respectively. A nearly linear relationship exists between the dielectric displacement and the electric field when the electric field is weak. When considering nanosecond three-wave parametric interactions, only the second order nonlinear term (2.9) will be included in the nonlinear polarization. This approximation is justified by the negligible contribution of the third and higher orders of polarization in the intensity regime below $\sim 1 \mathrm{GW} / \mathrm{cm}^{2}$, common for solid-state materials and nanosecond pulses. 
The assumption of spatial uniformity and instantaneity of the dielectric response results in a simplification of relations $(2.8-2.9)$ :

$$
\begin{gathered}
\mathbf{P}^{(1)}(\mathbf{r}, t)=\varepsilon_{0} \chi^{(1)} \mathbf{E}(\mathbf{r}, t), \\
\mathbf{P}^{(2)}(\mathbf{r}, t)=\chi^{(2)} \mathbf{E}(\mathbf{r}, t) \mathbf{E}(\mathbf{r}, t),
\end{gathered}
$$

where

$$
\begin{gathered}
\chi^{(1)}\left(\mathbf{r}-\mathbf{r}^{\prime}, t-\tau\right)=\chi^{(1)} \delta(\tau) \\
\chi^{(2)}\left(\mathbf{r}-\mathbf{r}_{1}, t-\tau_{1} ; \mathbf{r}-\mathbf{r}_{2}, t-\tau_{2}\right)=\chi^{(2)} \delta\left(\tau_{1}\right) \delta\left(\tau_{2}\right) .
\end{gathered}
$$

In a dispersionless medium the displacement vector $\mathbf{D}$ can be decomposed into a linear $\left(\mathbf{D}^{(1)}\right)$ and nonlinear $\left(\mathbf{P}^{\mathrm{NL}}\right)$ part:

$$
\mathbf{D}(\mathbf{r}, t)=\mathbf{D}^{(1)}(\mathbf{r}, t)+\mathbf{P}^{\mathrm{NL}}(\mathbf{r}, t)=\varepsilon_{0} \mathbf{E}(\mathbf{r}, t)+\varepsilon_{0} \chi^{(1)} \mathbf{E}(\mathbf{r}, t)+\mathbf{P}^{\mathrm{NL}}(\mathbf{r}, t),
$$

which is further simplified in case of an isotropic material

$$
\mathbf{D}(t)=\varepsilon_{0}\left(1+\chi^{(1)}\right) \mathbf{E}(t)+\mathbf{P}^{\mathrm{NL}}(t)=\varepsilon_{0} \varepsilon_{r} \mathbf{E}(t)+\mathbf{P}^{\mathrm{NL}}(t) .
$$

The quantity $\varepsilon_{r}=1+\chi^{(1)}$ is often termed relative dielectric permeability in linear optics, while the total permeability of a dielectric material is $\varepsilon=\varepsilon_{0} \varepsilon_{r}$.

By taking a curl of Eq. (2.3) and substituting (2.4) and the constitutive relation (2.6), the assumption of no free current $(\mathrm{J}=0)$ gives

$$
\nabla \times \nabla \times \mathbf{E}(\mathbf{r}, t)+\mu_{0} \frac{\partial^{2} \mathbf{D}(\mathbf{r}, t)}{\partial t^{2}}=0
$$

Nonlinear polarization enters the wave equation by substituting (2.16) into (2.17):

$$
\nabla \times \nabla \times \mathbf{E}(\mathbf{r}, t)+\mu_{0} \varepsilon \frac{\partial^{2} \mathbf{E}(\mathbf{r}, t)}{\partial t^{2}}=-\mu_{0} \frac{\partial^{2} \mathbf{P}^{\mathrm{NL}}(\mathbf{r}, t)}{\partial t^{2}}
$$

The derived wave equation (2.18) is an inhomogeneous differential equation, with a distinct nonlinear drive term on the right hand side. The solution of the homogeneous left 
hand side of (2.18) is the usual electromagnetic wave propagating through a dielectric medium with a phase velocity $1 / \sqrt{\varepsilon \mu_{0}}=c / \sqrt{\varepsilon_{r}}$.

Since the following discussion includes broad bandwidth pulses at several different central frequencies, it is important to derive the frequency-dependent form of the wave equation. This can be done by decomposing the electric field, the linear electric displacement and the nonlinear polarization into their frequency components:

$$
\begin{gathered}
\mathbf{E}(\mathbf{r}, t)=\sum_{j} \mathbf{E}_{j}(\mathbf{r}, t)=\sum_{j} \mathbf{E}_{j}(\mathbf{r}) \exp \left(-i \omega_{j} t\right)+c . c . \\
\mathbf{D}^{(1)}(\mathbf{r}, t)=\sum_{j} \mathbf{D}_{j}^{(1)}(\mathbf{r}, t)=\sum_{j} \mathbf{D}_{j}^{(1)}(\mathbf{r}) \exp \left(-i \omega_{j} t\right)+c . c . \\
\mathbf{P}^{\mathrm{NL}}(\mathbf{r}, t)=\sum_{j} \mathbf{P}_{j}^{\mathrm{NL}}(\mathbf{r}, t)=\sum_{j} \mathbf{P}_{j}^{\mathrm{NL}}(\mathbf{r}) \exp \left(-i \omega_{j} t\right)+c . c .
\end{gathered}
$$

Linear electric displacement is obtained from the electric field and the frequencydependent dielectric tensor $\varepsilon^{(1)}(\omega)$ :

$$
\mathbf{D}_{j}^{(1)}(\mathbf{r})=\varepsilon^{(1)}(\omega) \mathbf{E}_{j}(\mathbf{r})=\varepsilon_{0}\left(\mathbf{1}+\chi^{(1)}(\omega)\right) \mathbf{E}_{j}(\mathbf{r})
$$

Here, the frequency-dependent linear susceptibility tensor $\chi^{(1)}(\omega)$ is introduced, defined by the Fourier transform of the time-dependent susceptibility tensor:

$$
\chi^{(1)}(\omega)=\int_{-\infty}^{\infty} \chi^{(1)}(t) \exp (-i \omega t) d t
$$

The frequency decomposition $(2.19-2.21)$ can be now substituted into the wave equation (2.18) to obtain a set of wave equations for different frequency components:

$$
\nabla \times \nabla \times \mathbf{E}_{j}(\mathbf{r}, t)+\mu_{0} \varepsilon^{(1)}\left(\omega_{j}\right) \frac{\partial^{2} \mathbf{E}_{j}(\mathbf{r}, t)}{\partial t^{2}}=-\mu_{0} \frac{\partial^{2} \mathbf{P}_{j}^{\mathrm{NL}}(\mathbf{r}, t)}{\partial t^{2}}
$$

Finally, decomposition into Fourier components (2.19-2.21) can be included explicitly to eliminate the time dependence: 


$$
\nabla \times \nabla \times \mathbf{E}_{j}(\mathbf{r})-\omega_{j}^{2} \mu_{0} \varepsilon^{(1)}\left(\omega_{j}\right) \mathbf{E}_{j}(\mathbf{r})=\omega_{j}^{2} \mu_{0} \mathbf{P}_{j}^{\mathrm{NL}}(\mathbf{r})
$$

Eq (2.25) can be simplified if we use the vector identity $\nabla \times \nabla \times \mathbf{E}=\nabla(\nabla \cdot \mathbf{E})-\nabla^{2} \mathbf{E}$. In the case of plane waves, the term $\nabla(\nabla \cdot \mathbf{E})$ vanishes, leaving the wave equation in the form of an inhomogeneous Helmholtz equation:

$$
-\nabla^{2} \mathbf{E}_{j}(\mathbf{r})-\omega_{j}^{2} \mu_{0} \varepsilon^{(1)}\left(\omega_{j}\right) \mathbf{E}_{j}(\mathbf{r})=\omega_{j}^{2} \mu_{0} \mathbf{P}_{j}^{\mathrm{NL}}(\mathbf{r})
$$

Since every beam can be written as a superposition of plane waves, a set of inhomogeneous Helmholtz equations is adequate for the subsequent analysis of parametric interactions.

\subsection{Coupled wave equations for difference-frequency generation}

The wave equation (2.26) derived in section 2.1. can be used to describe the propagation of simple plane waves in a nonlinear medium. Most importantly, it can treat simultaneous propagation of waves comprised of different frequency components. A multitude of nonlinear effects involving several waves can be described by systems of coupled nonlinear wave equations.

Second-order $\left(\chi^{(2)}\right)$ nonlinear optical interactions ${ }^{14}$ include second-harmonic generation (SHG), sum-frequency generation (SFG), difference-frequency generation (DFG) and optical rectification (OR) - see Fig. 2.1. When the nonlinear wave equation is used to describe the propagation of waves coupled through third-order nonlinearity $\left(\chi^{(3)}\right)$, effects such as stimulated Brillouin scattering (SBS), stimulated Raman scattering (SRS) and self-phase modulation (SPM) can be treated. ${ }^{15}$ 
Optical parametric amplification ${ }^{16}$ is a process identical to DFG. Historically, DFG has been used to describe the mixing of three waves, where the two input waves have comparable intensities. The term optical parametric amplification, on the other hand, is used to describe the situation when the pump wave exhibits orders of magnitude greater intensity than the signal wave on the input face of the nonlinear medium. Since there is no fundamental difference between the two processes, particularly when the pump wave is appreciably depleted, both terms are used interchangeably in this work.

(a)

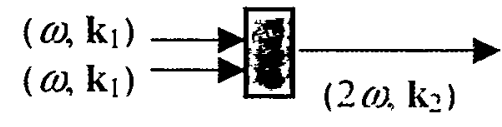

(c)

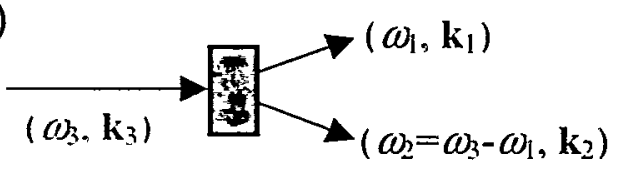

(b)

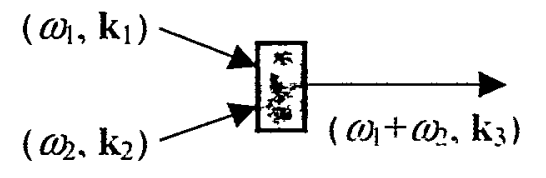

(d)

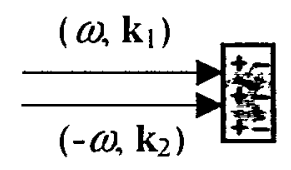

Figure 2.1. Second-order nonlinear optical interactions: (a) second-harmonic generation (SHG), (b) sum-frequency generation (SFG), (c) difference-frequency generation (DFG), (d) optical rectification (OR).

Before the coupled wave equations for DFG are derived, it is necessary to determine the nonlinear source term for DFG. For the two interacting fields $E_{j}$ and $E_{k}$ at frequencies $\omega_{j}$ and $\omega_{k}$, respectively, the second order nonlinear polarization (2.12) takes a form

$$
\begin{gathered}
\frac{P^{(2)}(\mathbf{r}, t)}{\varepsilon_{0}}=\chi^{(2)}\left(E_{j}^{2}(\mathbf{r}, t) \exp \left(-2 i \omega_{j} t\right)+E_{k}^{2}(\mathbf{r}, t) \exp \left(-2 i \omega_{k} t\right)+\right. \\
\left.+2 E_{j}(\mathbf{r}, t) E_{k}(\mathbf{r}, t) \exp \left(-i\left(\omega_{j}+\omega_{k}\right) t\right)+2 E_{k}^{*}(\mathbf{r}, t) E_{j}(\mathbf{r}, t) \exp \left(-i\left(\omega_{k}-\omega_{j}\right) t\right)+c . c .\right)+ \\
+2 \chi^{(2)}\left(E_{j}(\mathbf{r}, t) E_{j}^{*}(\mathbf{r}, t)+E_{k}(\mathbf{r}, t) E_{k}^{*}(\mathbf{r}, t)\right)
\end{gathered}
$$


The component of nonlinear polarization that is responsible for DFG of frequency $\omega_{l}=\omega_{k}$ $\omega_{f}$ is

$$
P_{l}(\mathbf{r}, t)=2 \varepsilon_{0} \chi^{(2)} E_{k}^{*}(\mathbf{r}, t) E_{j}(\mathbf{r}, t) \exp \left(-i\left(\omega_{k}-\omega_{j}\right) t\right)+c . c .
$$

Effective nonlinearity $d_{e f f}$ is defined by the usual relation $d_{e f f}=\chi^{(2)} / 2$.

It is assumed that the input fields in DFG are given by infinite plane waves at frequencies $\omega_{1}$ (signal) and $\omega_{3}$ (pump), generating a wave at a difference frequency $\omega_{2}=\omega_{3}-\omega_{1}$ (idler). All waves are assumed to be collinear, propagating in $\hat{z}$ direction in the lab reference frame, and can be described by

$$
E_{j}(z, t)=A_{j}(z) \exp \left(i\left(k_{j} z-\omega_{j} t\right)\right)+c . c ., j=1,2,3 .
$$

The nonlinear source term for DFG at the signal frequency is

$$
P_{1}(z, t)=P_{1}(z) \exp \left(-i \omega_{1} t\right)+c . c .=4 \varepsilon_{0} d_{e f f} A_{2}^{*}(z) A_{3}(z) \exp \left(i\left(\left(k_{3}-k_{2}\right) z-\omega_{1} t\right)\right)+c . c .
$$

Substitution of (2.30) into the wave equation (2.26) for signal yields

$$
\begin{aligned}
& \left(\frac{d^{2} A_{1}(z)}{d z^{2}}+2 i k_{1} \frac{d A_{1}(z)}{d z}-k_{1}^{2} A_{1}(z)+\omega_{1}^{2} \mu_{0} \varepsilon^{(1)}\left(\omega_{1}\right) A_{1}(z)\right) \exp \left(i\left(k_{1} z-\omega_{1} t\right)\right)+c . c .= \\
& \left.-4 d_{e f f} \frac{\omega_{1}^{2}}{\mathrm{c}^{2}} A_{2}^{*}(z) A_{3}(z) \exp \left(i\left(k_{3}-k_{2}\right) z-\omega_{1} t\right)\right)+c . c .
\end{aligned}
$$

Using the identity $k^{2}=\omega^{2} \mu_{0} \varepsilon^{(1)}$ and dropping the complex conjugate terms and collecting the exponential terms on both sides simplifies the equation to

$$
\frac{d^{2} A_{1}(z)}{d z^{2}}+2 i k_{1} \frac{d A_{1}(z)}{d z}=-4 d_{e f f} \frac{\omega_{1}^{2}}{\mathrm{c}^{2}} A_{2}^{*}(z) A_{3}(z) \exp \left(i\left(k_{3}-k_{1}-k_{2}\right) z-\omega_{1} t\right)
$$

Using the slowly varying amplitude approximation $\left(\left|d^{2} A / d z^{2}\right| \ll|k d A / d z|\right)$, the second derivative term in (2.32) can be dropped, giving the final wave equation for signal: 


$$
\frac{d A_{1}(z)}{d z}=i \frac{2 d_{e f f} \omega_{1}^{2}}{k_{1} c^{2}} A_{2}^{*}(z) A_{3}(z) \exp (i \Delta k z),
$$

where $\Delta k$ is the wave vector mismatch. Similar wave equations can be derived for idler and pump waves:

$$
\begin{aligned}
& \frac{d A_{2}(z)}{d z}=i \frac{2 d_{e f f} \omega_{2}^{2}}{k_{2} c^{2}} A_{1}^{*}(z) A_{3}(z) \exp (i \Delta k z), \\
& \frac{d A_{3}(z)}{d z}=i \frac{2 d_{e f f} \omega_{3}^{2}}{k_{3} c^{2}} A_{1}(z) A_{2}(z) \exp (-i \Delta k z) .
\end{aligned}
$$

Equations (2.33)-(2.35) represent the final system of coupled differential equations for field amplitudes of signal, idler and pump waves in DFG, with the approximations and assumptions introduced above.

\subsection{Solutions of wave equations for optical parametric amplification with nanosecond pulses}

Under special conditions, the system of coupled wave equations (2.33-2.35) can be solved analytically. The first analytical solution was given by Armstrong et al. ${ }^{17}$ Following their treatment, absorption is neglected and an assumption is made that the interacting fields consist of infinite plane waves. A simplified solution, which does not

include pump depletion, is derived first. In this case, $A_{3}=$ const and Eq. (2.33) is differentiated to obtain

$$
\frac{d^{2} A_{1}(z)}{d z^{2}}=-\Delta k \frac{2 \omega_{1}}{n_{1} c} d_{e f f} \frac{d A_{2}^{*}(z)}{d z} A_{3} \exp (i \Delta k z)
$$

$d A_{2}^{*} / d z$ can now be eliminated from (2.36) by substitution of (2.34): 


$$
\frac{d^{2} A_{1}(z)}{d z^{2}}-i \Delta k \frac{4 d_{e f}^{2} \omega_{1} \omega_{2}}{c^{2} n_{1} n_{2}}\left|A_{3}\right|^{2} A_{1}(z)=0
$$

With a definition

$$
\kappa_{1,2}=\frac{2 i d_{e f f} \omega_{1,2} A_{3}}{c n_{1,2}}, g=\sqrt{\kappa_{1} \kappa_{2}^{*}-(\Delta k / 2)^{2}},
$$

the equation for the field $A_{1}$ is

$$
\frac{d^{2} A_{1}(z)}{d z^{2}}+i \Delta k \kappa_{1} \kappa_{2}\left|A_{3}\right|^{2} A_{1}(z)=0 .
$$

For a complete set of boundary conditions for signal and idler, the solution is: ${ }^{15}$

$$
\begin{aligned}
& A_{1}(z)=\left(A_{1}(0)\left(\cosh g z-\frac{i \Delta k}{2 g} \sinh g z\right)+\frac{\kappa_{1}}{g} A_{2}^{*}(0) \sinh g z\right) \exp \left(\frac{i \Delta k z}{2}\right), \\
& A_{2}(z)=\left(A_{2}(0)\left(\cosh g z-\frac{i \Delta k}{2 g} \sinh g z\right)+\frac{\kappa_{2}}{g} A_{1}^{*}(0) \sinh g z\right) \exp \left(\frac{i \Delta k z}{2}\right) .
\end{aligned}
$$

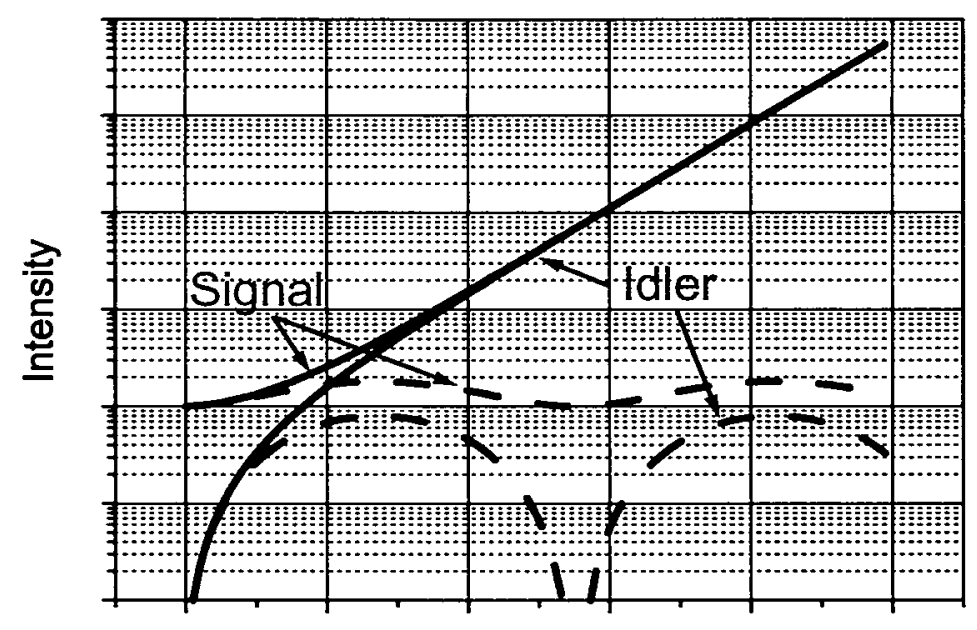

\section{Crystal length}

Figure 2.2. Qualitative behavior of the solutions for signal and idler in OPA operating in the small signal regime. Solid lines indicate the solution without dephasing, while dashed lines correspond to the solution for $\Delta k=3 \kappa_{1} \kappa_{2}{ }^{*}$. No incident idler is present. 
In Fig. 2.2, the solution is presented for two sets of initial conditions. It is important to note the phase sensitivity of optical parametric amplification with respect to the incident idler. In the common case of small-signal optical parametric amplification, the incident idler is absent $\left(A_{2}(0)=0\right)$. In that case, the solutions for small signal optical parametric amplification reduce to

$$
\begin{gathered}
A_{1}(z)=A_{1}(0)\left(\cosh g z-\frac{i \Delta k}{2 g} \sinh g z\right) \exp \left(\frac{i \Delta k z}{2}\right), \\
A_{2}(z)=\frac{\kappa_{2}}{g} A_{1}^{*}(0) \sinh g z \exp \left(\frac{i \Delta k z}{2}\right) .
\end{gathered}
$$

An important aspect of the solution is the fact that the fields of the signal, idler and pump are coupled and the amplification of the signal cannot be effectively be decoupled from the idler amplification (through, for example, idler absorption).

Armstrong et al. ${ }^{17}$ derived the general solution for DFG with pump depletion. Their result for signal and idler contains elliptical sine functions, and can be written in a convenient form for signal gain $G$ :

$$
\begin{gathered}
G=1+\frac{\lambda_{3}}{\lambda_{1}} \frac{I_{3}}{I_{1}}\left(1-\operatorname{sn}^{2}\left(N_{0} g L-\frac{K\left(N_{0}\right)}{\sqrt{N_{0}}}, N_{0}\right)\right) \operatorname{sinc}^{2}\left(\frac{\Delta k L}{2}\right), \\
N_{0}=\left(1+\frac{I_{1}}{I_{3}} \frac{\lambda_{1}}{\lambda_{3}}\right)^{-1} .
\end{gathered}
$$

A qualitative solution for the pump conversion to signal and idler is shown in Fig. 2.3. An important relation among the intensities of the signal, idler and pump wave can be derived from the coupled wave equations (2.33-2.35):

$$
\frac{d}{d z}\left(\frac{I_{1}}{\omega_{1}}\right)=\frac{d}{d z}\left(\frac{I_{2}}{\omega_{2}}\right)=-\frac{d}{d z}\left(\frac{I_{3}}{\omega_{3}}\right) .
$$


This relation is called Manley-Rowe relation, ${ }^{18}$ and it indicates that the rate of the creation of the signal and idler photons is equal to the rate of destruction of pump photons in a lossless medium. This is fully consistent with a full quantum mechanical description of the parametric process.

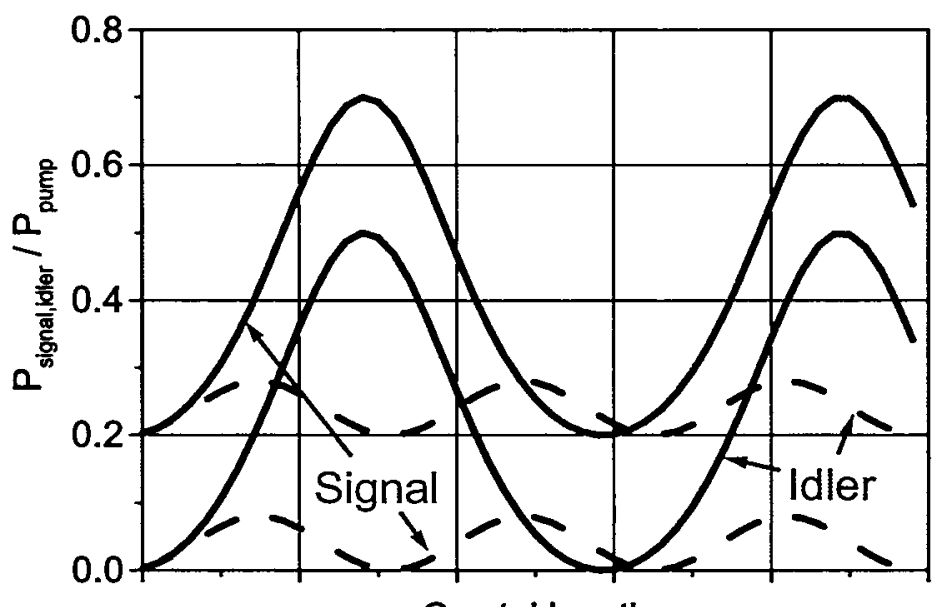

Crystal length

Figure 2.3. Qualitative behavior of the solutions for signal and idler in OPA operating in the regime of depleted pump. Solid lines indicate the solution without dephasing, while dashed lines correspond to the solution for $\Delta k=3 \kappa_{1} \kappa_{2}^{*}$. No incident idler is present, while the initial condition for the signal is $P_{\text {signat }}(0) / P_{\text {pump }}(0)=0.2$.

\subsection{Phase matching}

The flow of energy among the pump, signal and idler waves is sensitive to their phase relationship. Maximum conversion is achieved when that phase relationship is constant and equals $\phi_{3}-\phi_{1}-\phi_{2}=-\pi / 2$. A change of the phase relationship among the waves causes the energy to flow back to the pump wave before the pump energy has been completely depleted. The change of phase occurs over a distance which is called the 
coherence length $l_{c}=1 / \Delta \mathrm{k}$. The requirement for phase matching can be written in terms of wave vectors for the three waves:

$$
\mathbf{k}_{1}+\mathbf{k}_{2}=\mathbf{k}_{3}
$$

In the case the waves are collinear, the requirement (2.47) has a simple scalar equivalent

$$
k_{1}+k_{2}=k_{3}
$$

where $k_{1}=\left|\mathbf{k}_{1}\right|, k_{2}=\left|\mathbf{k}_{2}\right|$, and $k_{3}=\left|\mathbf{k}_{3}\right|$. The cases of collinear and noncollinear phase matching are shown schematically in Fig. 2.4 .

(a)

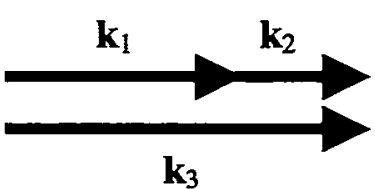

(b)

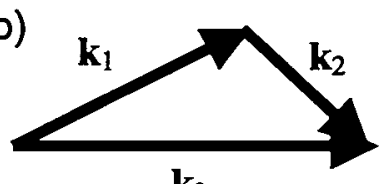

$\mathbf{k}_{3}$

Figure 2.4. (a) Collinear phase matching, (b) noncollinear phase matching.

In a dispersionless medium, phase matching would be achieved naturally for any combination of collinear wave vectors, since it would be identical to the conservation of energy. However, all media exhibit dispersive properties, resulting in a mismatch in phase velocities. Moreover, normal material dispersion prevents the phase matching to be achieved by using solely noncollinear geometry in a non-birefringent medium. This can be seen by considering the relationship between the refractive indices for normal dispersion: $n\left(\lambda_{1}\right)<n\left(\lambda_{2}\right)$ for $\lambda_{1}>\lambda_{2}$. In a noncollinear geometry we have $n_{1} / \lambda_{1}+n_{2} / \lambda_{2} \geq n_{3} / \lambda_{3}$, which is impossible to satisfy for the conservation of energy requirement $1 / \lambda_{1}+1 / \lambda_{2}=1 / \lambda_{3}$ and the wavelength relationship among three waves $\left(\lambda_{1}\right.$, $\lambda_{2}>\lambda_{3}$ ). In an effort to alleviate this problem, different phase matching schemes ${ }^{19}$ have been invented to permit a long coherence length in parametric processes. The three most 
popular phase matching techniques in nonlinear optics are briefly described here, along with some examples.

\section{Angular phase matching}

Angular phase matching was the first experimentally demonstrated phase matching technique. ${ }^{20,21}$ This method utilizes birefringent materials, which exhibit different refractive indices for different polarizations. Phase matching can be achieved for a particular combination of polarizations used for the signal, idler and pump waves propagating in a specific direction through an anisotropic material. Typically, one or two of the waves is in the ordinary polarization state, experiencing a constant refractive index irrespective of the propagation direction. The other waves are polarized orthogonally, and their refractive indices are functions of the propagation angle with respect to the crystal axes. These waves are called extraordinary waves. Two types of angular phase matching can be designated for DFG: type I corresponds to the case when the signal and the idler wave have the same polarization, while type II corresponds to the case when they have orthogonal polarization. For instance:

$$
\begin{aligned}
& \text { Type I: signal (o) + idler (o) = pump (e); } \\
& \text { Type II: signal (e) + idler (o) = pump (e), }
\end{aligned}
$$

where "o" denotes ordinary and " $\mathrm{e}$ " denotes extraordinary polarization. In uniaxial crystals, two characteristic refractive indices can be identified: $n_{o}$ (ordinary) and $n_{e}$ (extraordinary). An extraordinary wave propagating at an angle $\theta$ with respect to the crystal $\hat{z}$-axis in the principal plane of the crystal has an effective refractive index $n_{e}(\theta)$, which is a combination of the two characteristic refractive indices. Analytical solutions 
can be usually derived for different types of phase matching in uniaxial and biaxial crystals. Typical angular phase matching curves calculated for collinear DFG in betabarium borate $(\mathrm{BBO})^{22,23}$ and potassium dihydrogen phosphate $(\mathrm{KDP})^{24}$ are shown in Fig. 2.5.
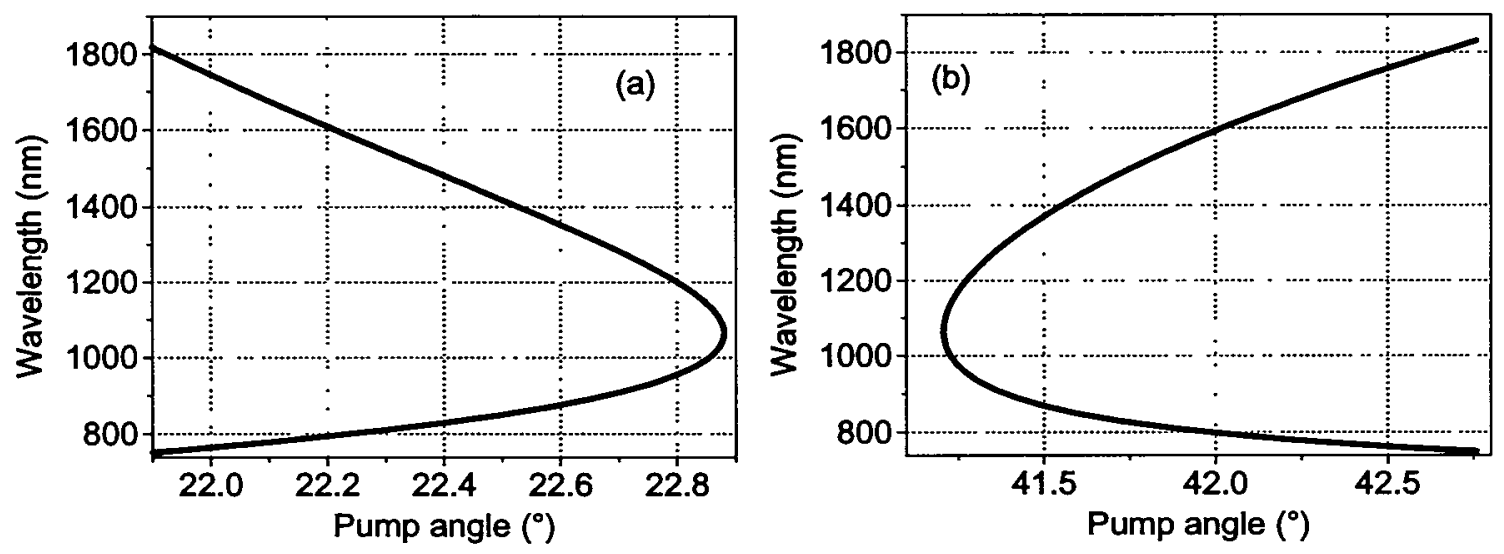

Figure 2.5. Angular phase matching curves for pump in (a) BBO, (b) KDP. The pump wavelength is $532 \mathrm{~nm}$. The mixing process is type I: signal (o) + idler (o) = pump (e). The geometry is collinear, and the Sellmeier coefficients are taken from references (a) 22, (b) 24.

\section{Temperature phase matching}

While angular tuning can be used to obtain critical phase matching (CPM), phase matching along one of the principal crystal axes can be achieved by temperature tuning. ${ }^{25}$ Phase matching while propagating along one of the crystal axes is called noncritical phase matching (NCPM). NCPM has an advantage over CPM in that no transverse walkoff of the beams occurs, resulting in an improved spatial overlap of the beams. NCPM can be achieved by using the temperature dependence of the refractive index in a nonlinear material. Generally, temperature phase matching can be also used in 
combination with CPM, particularly when it is more practical to modify and stabilize the crystal temperature than to implement a mechanism for fine angular tuning.

An example for temperature phase matching curve in lithium borate $(\mathrm{LBO})^{26}$ is shown in Fig. 2.6. While NCPM can be successfully used in OPAs pumped by divergent pump sources due to its large angular bandwidth, its application in high average power nonlinear optics remains limited by a narrow temperature bandwidth.

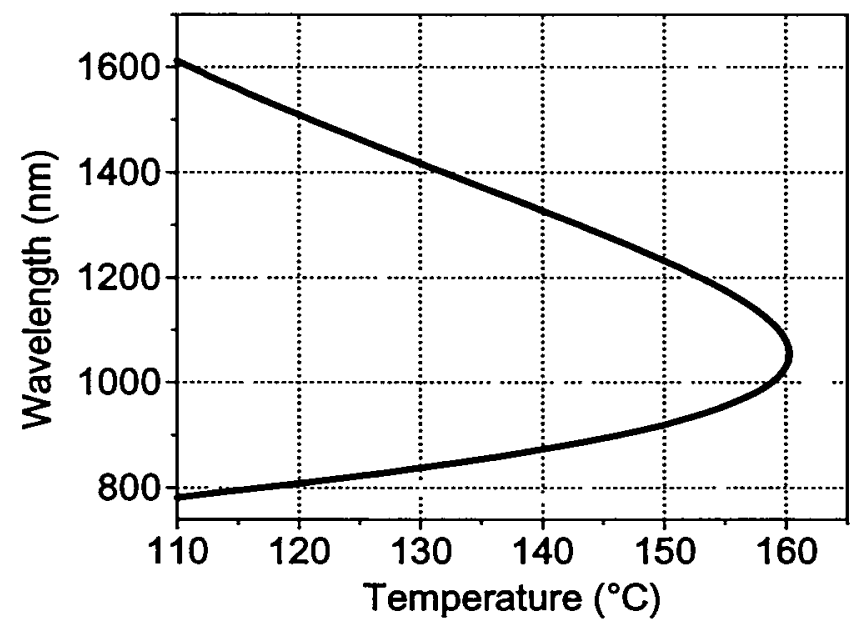

Figure 2.6. Temperature phase matching curves for NCPM LBO pumped at $532 \mathrm{~nm}$. The mixing process is type I: signal (o) + idler (o) $=$ pump (e). The Sellmeier coefficients are taken from reference 25 .

\section{Quasi-phase matching}

Quasi-phase matching (QPM) was the original proposed method for achieving phase matching, ${ }^{17}$ but it has been used experimentally only recently, ${ }^{27}$ as a consequence of the technological difficulties associated with manufacturing thin domains corresponding to short coherence lengths. The basic principle of QPM is the alternating sign of the nonlinear coefficient with a characteristic period of an integer multiple (usually one) coherence length in a periodically poled material (Fig. 2.7). Quasi-phase 
matching has several significant advantages compared to other phase matching methods. Wavelength tuning can be achieved simply by designing the right poling period to match the process coherence length (Fig. 2.8).

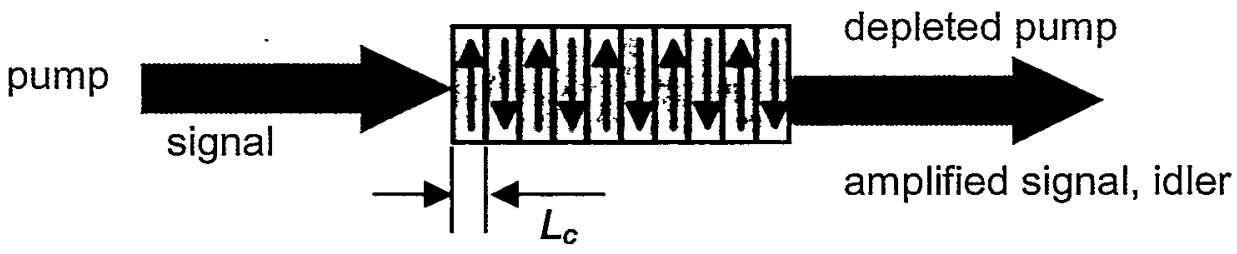

Figure 2.7. Periodically poled material has an alternating sign of effective nolinearity $d_{e f f}$, with a characteristic period of coherence length. The pump and signal beams are incident on the crystal perpendicular to the crystal axis and domain interface.

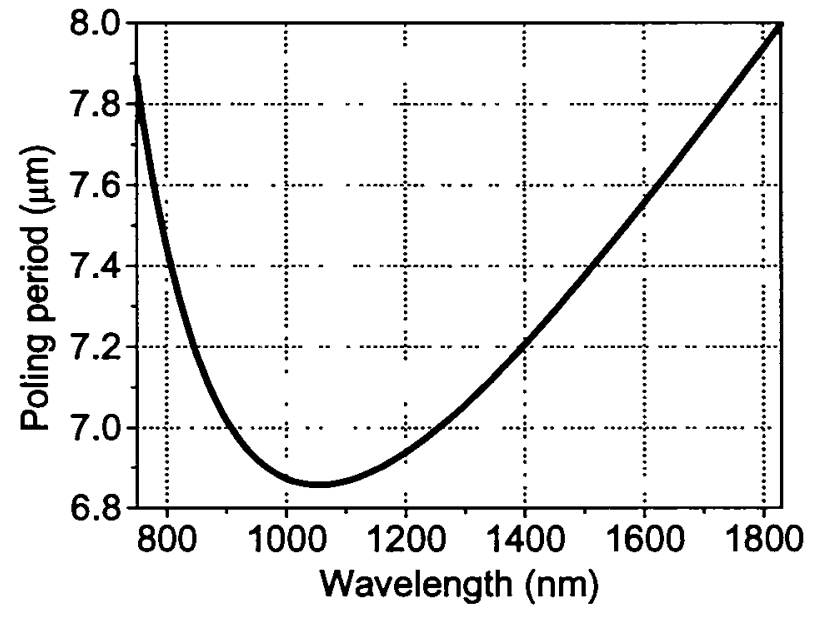

Figure 2.8. Poling period for QPM along $\hat{z}$ axis in lithium niobate as a function of signal wavelength. The pump is at 532 $\mathrm{nm}$, and the crystal is at a temperature of $293 \mathrm{~K}$.

Advantage can be taken of the crystal axis which produces maximum nonlinearity, allowing the crystal to be shorter for the same conversion efficiency. Transverse walk-off in the crystal is avoided by propagating along one of the crystal axes. Finally, the flexibility of poling allows for construction of monolithic devices which can perform 
additional operations such as pulse compression simultaneously with nonlinear conversion. $^{28,29}$ A disadvantage of the periodically poled materials is their limited aperture as a consequence of large electric field necessary for material poling. Despite these problems, approaches are being taken to increase the available aperture. ${ }^{30}$ The most common periodically poled materials today are PPLN (periodically poled lithium niobate) ${ }^{27}$ and PPKTP (periodically poled potassium triphosphate). ${ }^{31}$

\subsection{Light waves on the boundary of vacuum and nonlinear medium}

For a correct calculation of phase mismatch that occurs due to angular deviation of the beams, it is important to include the properties of the interface between vacuum and a medium that exhibits nonlinearity. Those properties were first studied by Bloembergen

and Pershan. ${ }^{32}$ In Fig. 2.9 the interacting waves on the boundary between the vacuum and a nonlinear medium are presented. A pump wave with wave vector $\mathbf{k}_{\mathbf{3 0}}$ and a signal wave with wave vector $\mathbf{k}_{\mathbf{1 0}}$ are incident on the boundary. The pump wave vector $\mathbf{k}_{\mathbf{3 0}}$ is chosen perpendicular to the boundary, and the signal is incident with an external noncollinear angle $\Omega$. A perfect phase matching is achieved in the crystal for the combination of transmitted signal $\mathbf{k}_{\mathbf{1 0}}$ ' and transmitted pump $\mathbf{k}_{\mathbf{3 0}}$, with the internal noncollinear angle $\Omega^{\prime}$. An incident idler with wave vector $\mathbf{k}_{20}$ conserves the nonlinear Snell's law $^{32}$ on the boundary and is transmitted as $\mathbf{k}_{\mathbf{2 0}}$ '. The amplitude of the incident idler is zero, which is consistent with the continuity of the idler wave on the boundary. The waves reflected on the boundary are not included, as they do not have relevance to optical parametric amplification. 


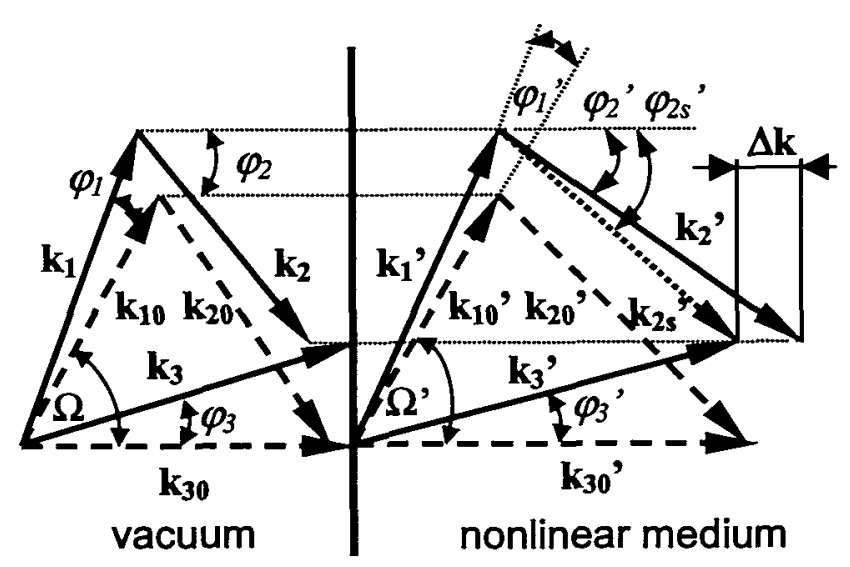

Figure 2.9. Waves on the boundary of vacuum (air) and nonlinear medium. The interface is perpendicular to the plane of paper.

Next, the wave vectors of the incident signal $\mathbf{k}_{\mathbf{1}}$ and pump $\mathbf{k}_{\mathbf{3}}$ are considered, with the associated transmitted signal wave vector $\mathbf{k}_{\mathbf{1}}$ ' and transmitted pump wave vector $\mathbf{k}_{\mathbf{3}}$ '. The wave vectors $\mathbf{k}_{1}$ and $\mathbf{k}_{\mathbf{3}}$ exhibit a small angular deviation from the ideally phasematched waves by the external angles $\varphi_{1}$ and $\varphi_{3}$, respectively. The refracted field angles and wave vectors $\left(\varphi_{1}, \varphi_{3}, \mathbf{k}_{1}{ }^{\prime}\right.$, and $\left.\mathbf{k}_{3}{ }^{\prime}\right)$ are calculated from the incident angles and wave vectors:

$$
\begin{gathered}
\sin \left(\Omega^{\prime}+\varphi_{1}{ }^{\prime}\right)=\frac{1}{n_{1}} \sin \left(\Omega+\varphi_{1}\right), \\
\sin \varphi_{3}^{\prime}=\frac{1}{n_{3}} \sin \varphi_{3}, \\
k_{1}^{\prime}=k_{1} n_{1}, \quad k_{2}^{\prime}=k_{2} n_{2}, \quad k_{3}^{\prime}=k_{3} n_{3},
\end{gathered}
$$

where $n_{1}, n_{2}$ and $n_{3}$ are the refractive indices of the transmitted signal, idler and pump. The solution for the idler wave in a nonlinear medium consists of a homogeneous and a particular solution. ${ }^{29}$ The driven idler wave $\mathbf{k}_{2 \mathbf{s}}$ ' corresponds to the homogeneous solution, while the transmitted idler $\mathbf{k}_{\mathbf{2}}$ ' corresponds to the particular solution. The 
transmitted idler $\mathbf{k}_{\mathbf{2}}$ ' is generated in the interaction. The incident idler angle $\varphi_{2}$, the driven idler angle $\varphi_{2 s}{ }^{\prime}$, and the transmitted idler angle $\varphi_{2}{ }^{\prime}$ can be extracted from the boundary conditions:

$$
\begin{gathered}
k_{2} \sin \varphi_{2}=k_{1} \sin \left(\Omega+\varphi_{1}\right)-k_{3} \sin \varphi_{3}, \\
k_{2 s}{ }^{\prime} \cos \varphi_{2 s}{ }^{\prime}=k_{3}{ }^{\prime} \cos \varphi_{3}{ }^{\prime}-k_{1}{ }^{\prime} \cos \left(\Omega^{\prime}+\varphi_{1}{ }^{\prime}\right), \\
\sin \varphi_{2}^{\prime}=\frac{1}{n_{2}} \sin \varphi_{2} .
\end{gathered}
$$

Finally, the phase mismatch is given by the difference between the transmitted idler wave and the driven idler wave:

$$
\Delta k=k_{2}^{\prime} \cos \varphi_{2}^{\prime}-k_{2 s}^{\prime} \cos \varphi_{2 s^{\prime}}
$$

In the paraxial limit, the exact expressions $(2.49-2.55)$ give a simple equation for wave vector mismatch:

$$
\begin{gathered}
\Delta k=n_{2} k_{2}\left(1-\frac{1}{2}\left(\frac{k_{1}\left(\Omega+\varphi_{1}\right)-k_{3} \varphi_{3}}{k_{2} n_{2}}\right)^{2}\right)-k_{3} n_{3}\left(1-\frac{1}{2}\left(\frac{\varphi_{3}}{n_{3}}\right)^{2}\right)+ \\
+k_{1} n_{1}\left(1-\frac{1}{2}\left(\frac{\Omega+\varphi_{1}}{n_{1}}\right)^{2}\right)
\end{gathered}
$$

The expression (2.56) for the wave vector mismatch is more convenient for numerical evaluation. It is sufficiently accurate for numerical modeling of wave vector mismatch between laser beams, where angular deviations are relatively small.

The next task is to determine the wave vector mismatch in biaxial crystals. Three distinct crystallographic axes $(X, Y, Z)$ can be defined for biaxial crystals. The refractive indices for fast and slow waves are the solutions of the general Fresnel equation: ${ }^{33}$ 


$$
\frac{\sin ^{2} \theta \cos ^{2} \phi}{1 / n^{2}-1 / n_{X}^{2}}+\frac{\sin ^{2} \theta \sin ^{2} \phi}{1 / n^{2}-1 / n_{Y}^{2}}+\frac{\cos ^{2} \theta}{1 / n^{2}-1 / n_{Z}^{2}}=0
$$

where $\theta$ and $\phi$ are the usual polar angles, and $n_{X}, n_{Y}$ and $n_{Z}$ are the refractive indices of the waves propagating along the respective crystallographic axes. The index of refraction in the direction defined by the angles $(\theta, \phi)$ is $n$. Departures from ideal phase matching are considered by tilting the beams in both angular directions. In the paraxial limit, the transmitted signal and pump wave angles are

$$
\begin{gathered}
\varphi_{1}^{\prime}=\frac{\varphi_{1}}{n_{1}}, \theta_{1}^{\prime}=\frac{\theta_{1}}{n_{1}}, \\
\varphi_{3}^{\prime}=\frac{\varphi_{3}}{n_{3}}, \theta_{3}^{\prime}=\frac{\theta_{3}}{n_{3}}
\end{gathered}
$$

The incident and the transmitted idler wave angles are

$$
\begin{gathered}
\varphi_{2}=\frac{k_{3} \varphi_{3}-k_{1} \varphi_{1}}{k_{2}}, \theta_{2}=\frac{k_{3} \theta_{3}-k_{1} \theta_{1}}{k_{2}} \\
\varphi_{2}^{\prime}=\frac{\varphi_{2}}{n_{2}}, \theta_{2}^{\prime}=\frac{\theta_{2}}{n_{2}} .
\end{gathered}
$$

A self-consistent calculation can be performed to obtain the transmitted angles from the incident angles. This is important because the refractive indices themselves are functions of transmitted angles. The driven idler wave is

$$
k_{2 s}^{\prime} \cos \varphi_{2 s}^{\prime} \cos \theta_{2 s}^{\prime}=k_{3}^{\prime} \cos \varphi_{3}^{\prime} \cos \theta_{3}^{\prime}-k_{1}^{\prime} \cos \varphi_{1}^{\prime} \cos \theta_{1}^{\prime} .
$$

The wave vector mismatch is

$$
\Delta k=k_{2}^{\prime} \cos \varphi_{2}^{\prime} \cos \theta_{2}^{\prime}-k_{2 s}^{\prime} \cos \varphi_{2 s}^{\prime} \cos \theta_{2 s}^{\prime}
$$

In Chapter 3, a calculation of angular bandwidth is performed for different nonlinear materials using the formalism presented in this section. 


\section{Chapter 3}

\section{Modeling of optical parametric}

\section{chirped pulse amplification}

Main advantages for the use of OPAs in CPA are substantiated by calculations presented in this Chapter. A brief overview of CPA is initially presented, including the motivation, basic principle, typical system components and prior experimental results. The concept of OPCPA is introduced and its background is presented in a similar fashion. The subsequent calculations illustrate the possibility of simultaneous large gain and broad amplified bandwidth in OPCPA. The calculations of spectral bandwidth and phase illustrate the feasibility of ultrashort pulse amplification and its high-fidelity recompression. Broad bandwidth regions are identified in several nonlinear materials. Temperature bandwidth calculation is performed to identify materials capable of handling high average power OPCPA. The calculation of angular bandwidth determines the practical requirements for pump lasers that are used in OPCPA. A simple calculation of pulse contrast in OPCPA is presented, which is of importance for certain classes of target experiments. In the end, the developed numerical model is described, which is used for OPCPA design in subsequent chapters. 


\subsection{Chirped pulse amplification (CPA)}

The pulse energy extracted from a laser is limited by the available volume of the pumped gain medium, which can maximally provide the energy stored in the population inversion. With the laser volume and aperture limited by the gain material mechanical characteristics, cost, and quality, another approach for producing high peak power is decreasing the pulse duration. With the advent of ultrashort pulse generation techniques such as mode-locking, ${ }^{6}$ direct amplification of ultrashort pulses to high energies in laser gain media became limited by the induced nonlinear effects and damage threshold of the laser medium. At high intensities associated with those pulses, the nonlinear component of the refractive index becomes more important:

$$
n=n_{0}+n_{2} I
$$

where $n$ is the total refractive index, $n_{0}$ is the refractive index in the absence of the external field, $n_{2}$ is the nonlinear refractive index, and $I$ is the laser pulse intensity. The origin of the nonlinear refractive index is in third-order susceptibility. In the usual case of positive nonlinear refractive index, more intense regions of the laser pulse (typically near the transverse beam center) experience a greater refractive index than the less intense regions. In effect, laser gain medium acts as a lens that distorts and focuses the beam. The result is gain medium damage by the runaway effect of self-focusing ${ }^{34}$ seeded by the most intense parts of the beam profile.

Nonlinear self-focusing limits the peak power obtainable directly from a solid-state laser aperture to intensities on the order of $\mathrm{GW} / \mathrm{cm}^{2}$. Chirped pulse amplification ${ }^{7}$ (CPA), first demonstrated in 1985, represents the enabling technology behind the production of 
extremes in peak power from lasers. ${ }^{35-37}$ The basic principle of CPA is shown schematically in Fig. 3.1. A CPA system starts with a short-pulse oscillator, which typically produces nJ-level pulses. It is followed by a pulse stretcher, which introduces a positive group delay on the spectral components of the broadband short pulse, effectively stretching it in time and chirping it. The low-energy stretched pulse is subsequently injected into a laser gain medium and amplified. Finally, the amplified chirped pulse is introduced into a compressor, which produces a negative group delay and recompresses the amplified pulse. The result of CPA is an energetic short pulse with otherwise unattainable level of peak power.

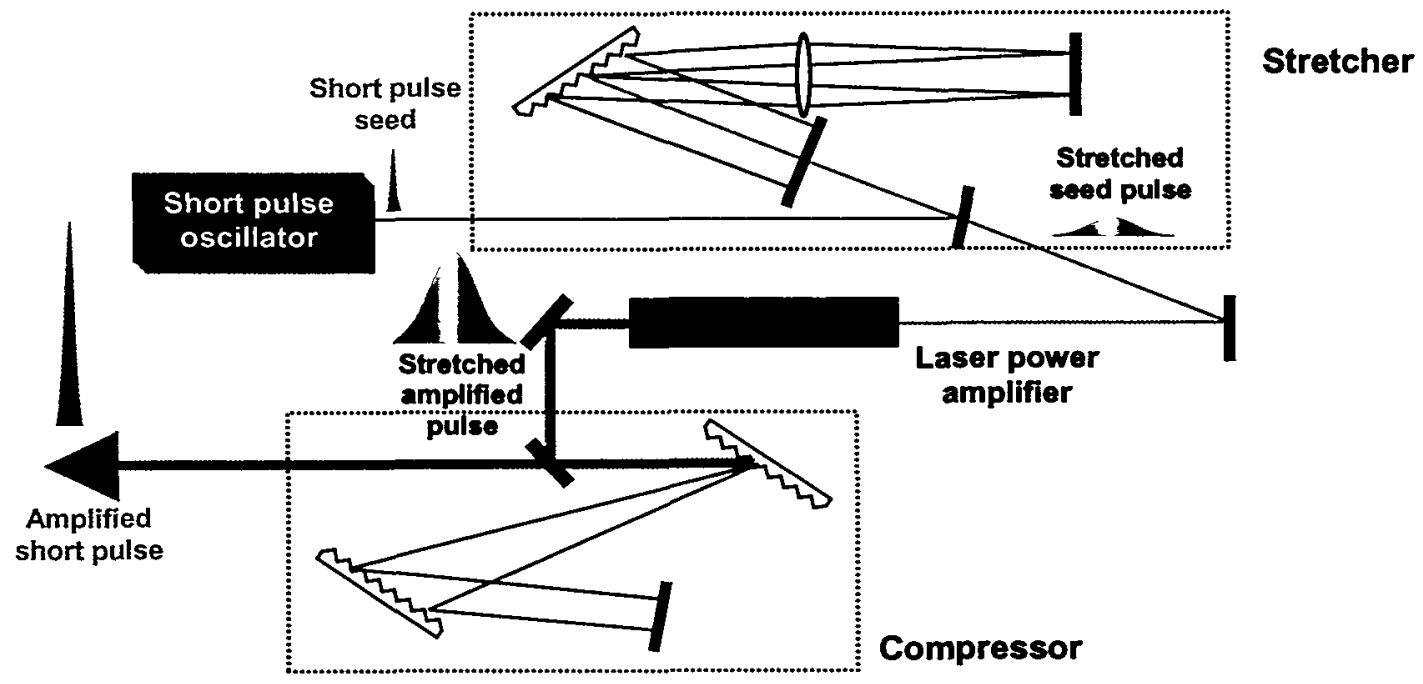

Figure 3.1. Chirped pulse amplification using a laser amplifier

The fundamental characteristic of CPA is that it allows high fluence level and high energy extraction from the laser amplifiers, while avoiding prohibitively high intensities and the resulting self-focusing problem. Typical designs of the stretcher-compressor pair involve components that induce angular dispersion, such as diffractive gratings and 
prisms, or linearly dispersive elements such as optical fibers. The compressor-stretcher design shown in Fig. 3.1 is based on the original Treacy-Martinez ${ }^{38-40}$ diffractive grating arrangement and is used extensively to provide large stretching ratios, typically $10^{3}-10^{4}$.

CPA using laser amplifiers has been successfully applied to develop tabletop 1 TW - $100 \mathrm{TW}$ systems, ${ }^{41,42}$ particularly those based on Ti:sapphire. Development of large aperture diffractive optics ${ }^{43}$ has enabled the extremes of power to be produced from large glass laser facilities utilizing CPA. The development recently culminated with more than a 1 PW of power obtained from a hybrid Ti:sapphire-Nd:glass CPA system. ${ }^{44}$

\subsection{Optical parametric chirped pulse amplification (OPCPA)}

The fundamental difference between a laser gain medium and an OPA is that a laser gain medium stores the integrated pump energy, whereas OPA relies on an instantaneous nonlinear process for amplification. In an alternative view, OPA can be regarded as a zero-lifetime laser gain medium. The absence of relaxation lifetime in OPA indicates that the pump must be present and temporally overlapped with the signal during the amplification process in OPA.

While conventional CPA uses laser amplifiers as gain media, optical parametric chirped pulse amplification (OPCPA) instead utilizes OPAs. The principle schematic in OPCPA is shown in Fig. 3.2. The important component of the OPCPA system is the pump laser and the precise timing system that ensures that the pump and signal pulses are overlapped in time during amplification in OPA. 


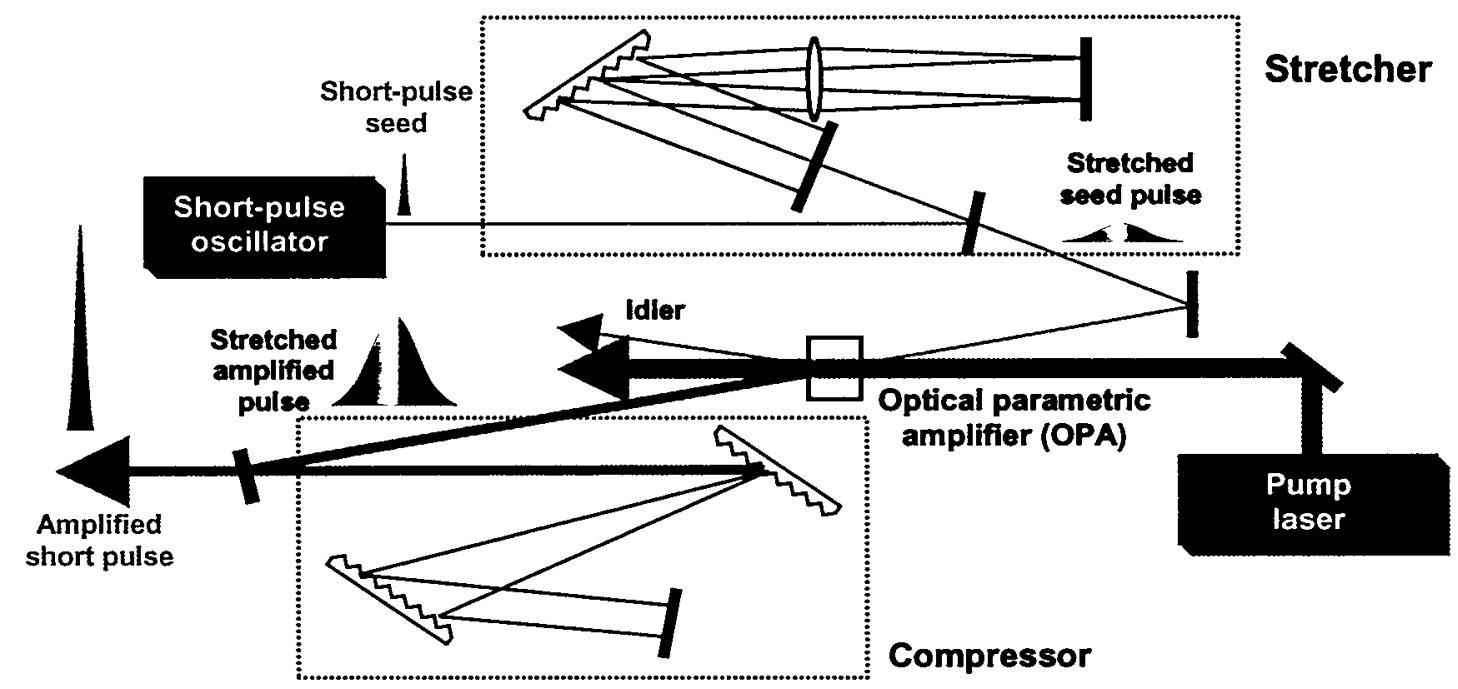

Figure 3.2. Optical parametric chirped pulse amplification

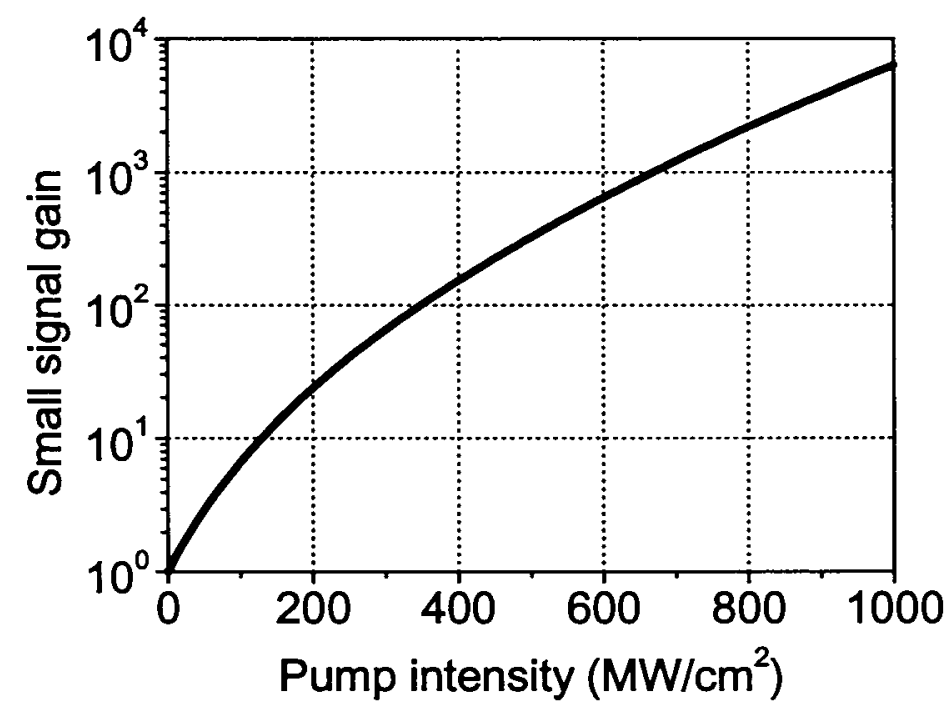

Figure 3.3. Single-pass gain in a $10 \mathrm{~mm}$ long $\mathrm{BBO}$ crystal; $\lambda_{s}=1054 \mathrm{~nm}, \lambda_{p}=532 \mathrm{~nm}$.

OPCPA was conceived in $1986,{ }^{45}$ while the first demonstration including pulse compression was done in 1992 by Dubietis et al. ${ }^{12}$ The concept has been subsequently expanded by Ross et al. ${ }^{13}$ to determine the practical limits of the technology in obtaining 
high peak power. Theoretical scaling arguments ${ }^{13,46}$ and preliminary designs ${ }^{13,47}$ were presented, suggesting that a maximum power of $15 \mathrm{PW}$ is possible using OPCPA and the current state-of-the-art compression grating technology.

OPCPA is a process which can proceed efficiently in the nanosecond pump regime, where the maximum intensities which are safely below damage threshold can be as high as $1 \mathrm{GW} / \mathrm{cm}^{2}$. Nonlinear materials are available which exhibit high enough nonlinearity to enable large gain from a relatively short $(\sim \mathrm{cm})$ crystal. As an example, small signal gain in BBO as a function of the pump intensity and crystal length is calculated and shown in Fig. 3.3. It is apparent that gain as high as $10^{3}-10^{4}$ can be obtained in a single pass through a BBO crystal which is only $10 \mathrm{~mm}$ long and is subjected to intensities of several hundred $\mathrm{MW} / \mathrm{cm}^{2}$, safely below the BBO damage threshold. ${ }^{48}$ This reveals one great advantage of using OPA in CPA: very large gain in OPA allows single pass amplification of the chirped signal pulse over many orders of magnitude, eliminating expensive and complicated multipass architectures of common CPA laser systems.

\subsection{OPA spectral bandwidth}

Conventional lasers typically exhibit relatively narrow spectral lines, which can in certain instances be broadened by processes such as the lifetime, phonon, Doppler and strain broadening. ${ }^{49}$ In some cases, the characteristics of the laser gain medium are particularly favorable for broadband amplification, such as in Ti:sapphire. ${ }^{50}$ In addition to large tunability, a very important feature of amplification in OPCPA is a large spectral bandwidth that is possible to obtain in type I phase-matched processes. 
In OPAs that operate near degeneracy $\left(\lambda_{s}=\lambda_{i}\right)$, broad bandwidth is achieved best in collinear geometry (see Fig. 3.4 for spectral bandwidth calculations of nearly degenerate OPA in several nonlinear crystals).
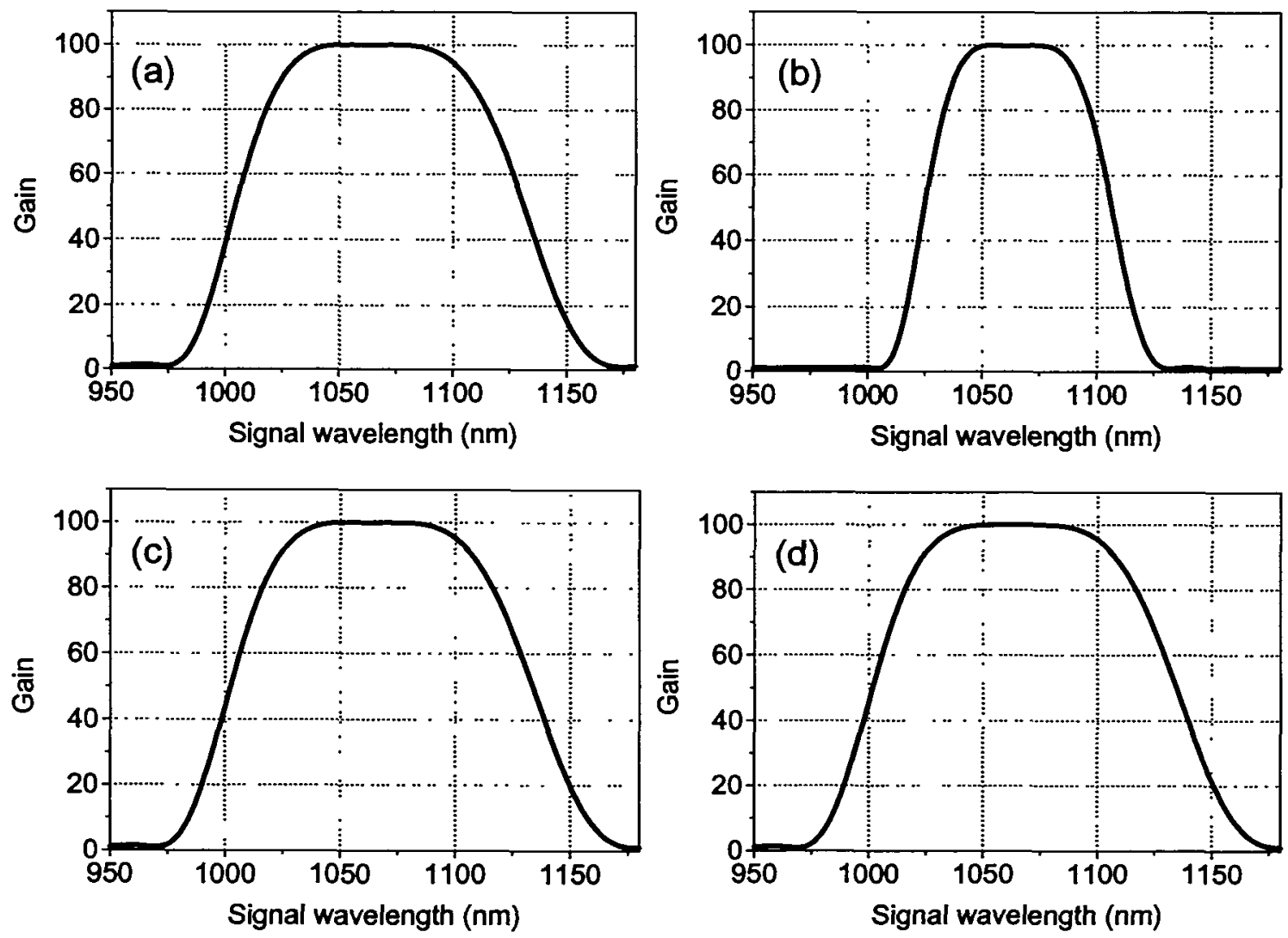

Figure 3.4. Nearly degenerate OPA spectral bandwidth for a) BBO, b) KDP, c) critically phase-matched LBO, and d) noncritically phase-matched LBO. The crystals are pumped by $\lambda_{p}=532 \mathrm{~nm}$ at an intensity of $500 \mathrm{MW} / \mathrm{cm}^{2}$. The length of the crystal is adjusted to produce a gain of $10^{2}$ at the signal center wavelength $\lambda_{s}=1054 \mathrm{~nm}$.

In OPAs that operate far from degeneracy, it is necessary to take advantage of noncollinear phase matching, which can dramatically increase the bandwidth in some instances. Fig. 3.5 shows the result of the spectral bandwidth calculation for 
nondegenerate OPA for a variety of noncollinear angles $\Omega$. It is important to note that, for a given wavelength of the pump, a certain-"magic" noncollinear angle exists which maximizes the OPA bandwidth around a certain favorable signal center wavelength.
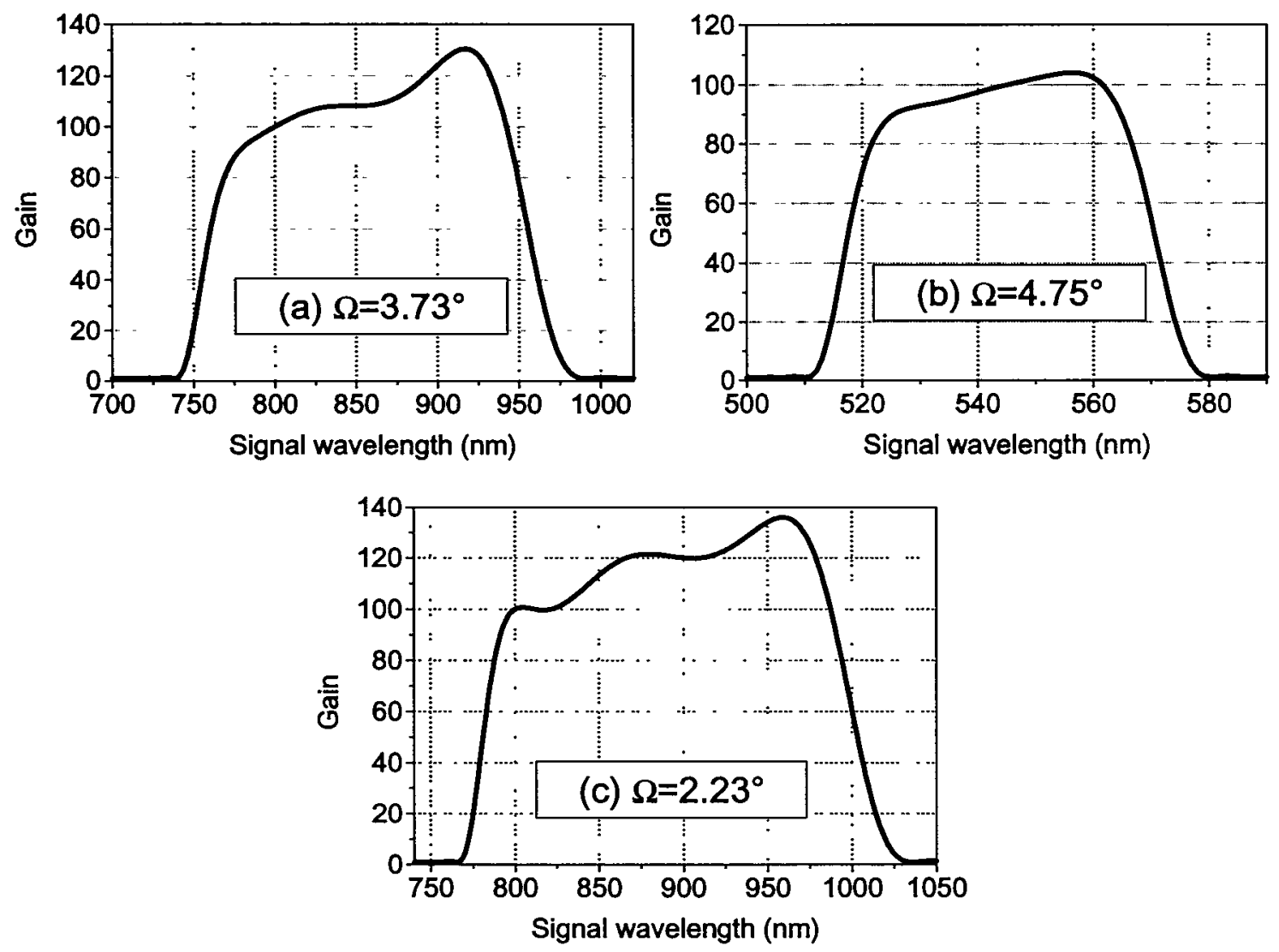

Figure 3.5. Nondegenerate OPA spectral bandwidth for (a) BBO, (b) KDP, (c) critically phase-matched LBO. The crystals in (a) and (c) are pumped by $\lambda_{p}=532 \mathrm{~nm}$, while the crystal in case (b) is pumped by $\lambda_{p}=351 \mathrm{~nm}$. Pump intensity is $500 \mathrm{MW} / \mathrm{cm}^{2}$. The length of the crystal is adjusted to produce a gain of $10^{2}$ at the signal center wavelength: (a) $\lambda_{s}=800 \mathrm{~nm}$, (b) $\lambda_{s}=550 \mathrm{~nm}$, (c) $\lambda_{s}=800 \mathrm{~nm}$.

Figure 3.5 suggests that, in addition to degeneracy, a very broad spectral bandwidth can be amplified at a center wavelength of $850 \mathrm{~nm}$ in $\mathrm{BBO}$ and $900 \mathrm{~nm}$ in LBO when those crystals are pumped at a wavelength of $532 \mathrm{~nm}$ (second harmonic of a Nd:glass laser). A 
very broad spectral bandwidth can be obtained from KDP at a center signal wavelength near $550 \mathrm{~nm}$ when the crystal is pumped at a wavelength of $351 \mathrm{~nm}$ (third harmonic of a Nd:glass laser).

\subsection{OPA spectral phase}

An important consideration in a broadband OPA is the measure of spectral phase imparted on the chirped pulse during amplification. The importance of the spectral phase stems from the requirement for high fidelity recompression of the amplified pulses in CPA. Since the final recompressed pulse is affected not only by the spectral amplitude bandwidth, but also by the spectral phase, it is important to identify the sources of spectral phase in OPA.

The origin of the spectral phase during amplification in conventional laser materials is usually the nonlinear refractive index (B-integral) ${ }^{51}$ associated with high beam intensity while propagating through a relatively long gain medium. In optical parametric amplification, the spectral phase is a result of the wave vector mismatch among different spectral components of signal, idler and pump waves. The calculation of the imparted phase on the amplified pulse across the spectral bandwidth indicates a correlation between the amplitude and phase parts of the spectral bandwidth. It can be also observed that the imparted phase is sufficiently small for femtosecond pulse amplification and follows a pattern which can be compensated. The calculation of spectral phase in collinear and noncollinear geometry is given in Figs. 3.6-3.7, accompanying the cases of amplitude bandwidth presented in Figs. 3.4-3.5. 

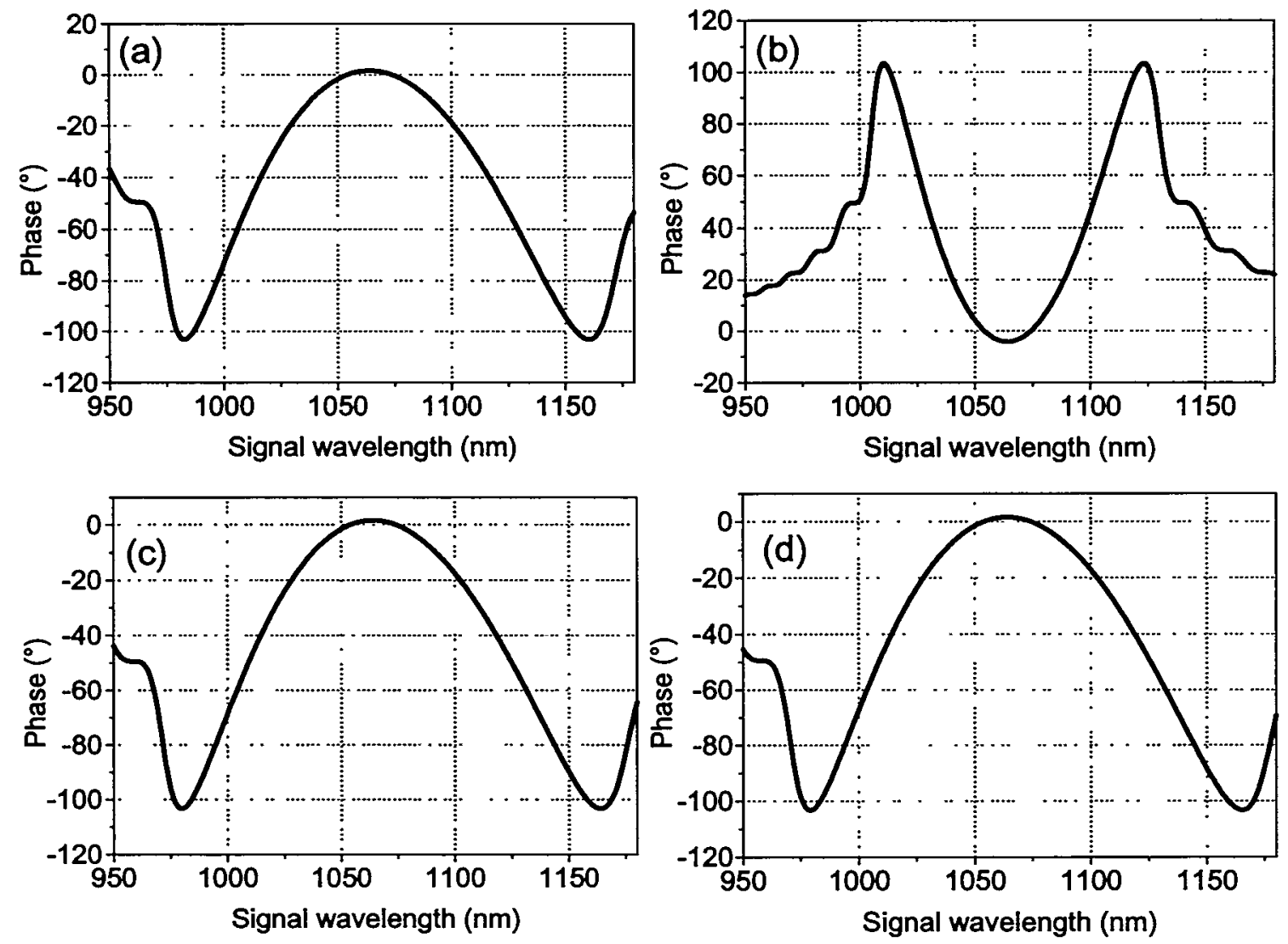

Figure 3.6. Nearly degenerate OPA spectral phase for (a) $\mathrm{BBO}$, (b) $\mathrm{KDP}$, (c) critically phase-matched LBO, and (d) NCPM LBO. The crystals are pumped by $\lambda_{p}=532 \mathrm{~nm}$ at an intensity of $500 \mathrm{MW} / \mathrm{cm}^{2}$. The length of the crystal is adjusted to produce a gain of $10^{2}$ at the signal center wavelength $\lambda_{s}=1054 \mathrm{~nm}$. 

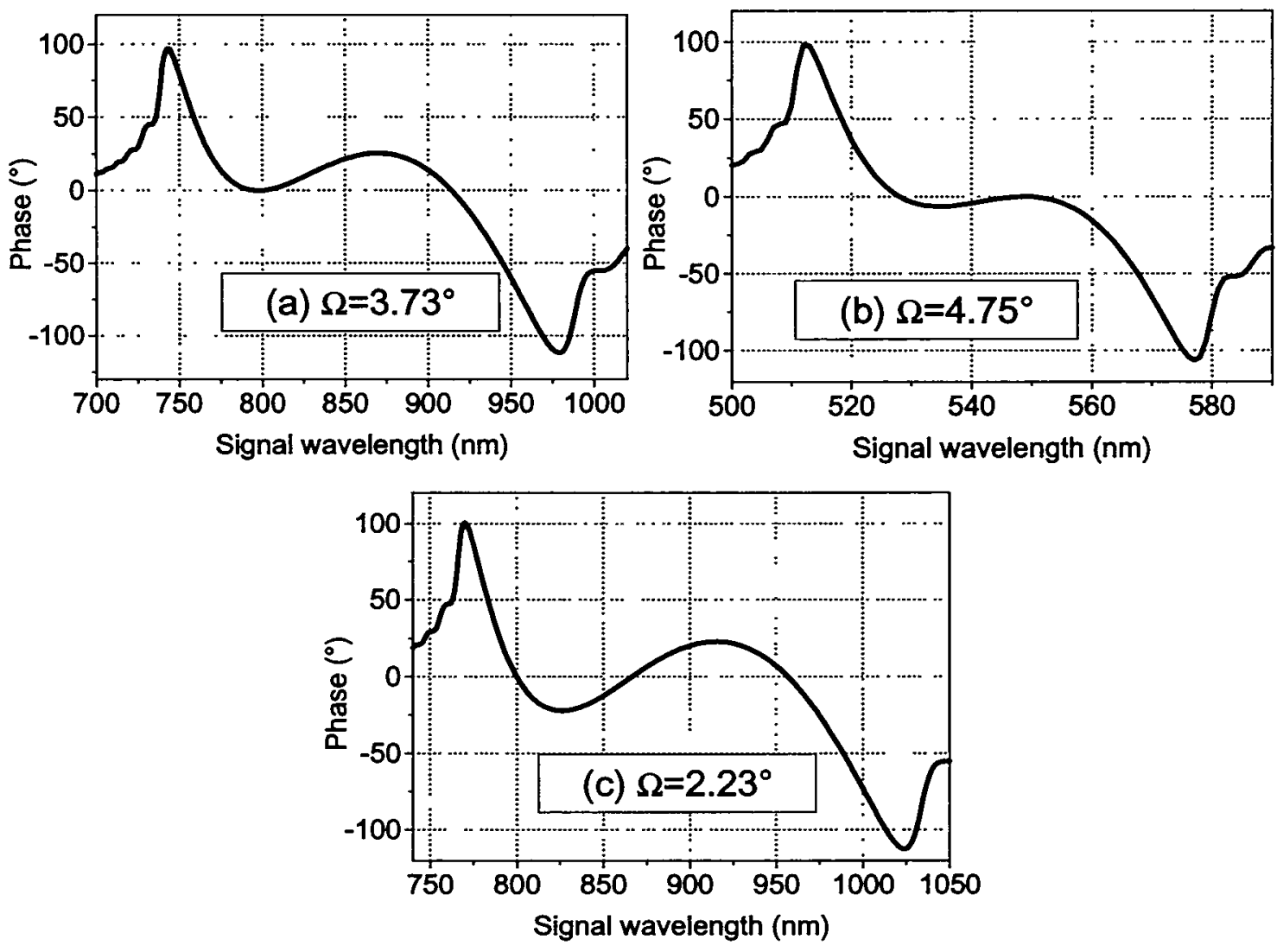

Figure 3.7 Nondegenerate OPA spectral phase for (a) BBO, (b) KDP, and (c) critically phase-matched LBO. The crystals in (a) and (c) are pumped by $\lambda_{p}=532 \mathrm{~nm}$, while the crystal in case (b) is pumped by $\lambda_{p}=351 \mathrm{~nm}$. Pump intensity is $500 \mathrm{MW} / \mathrm{cm}^{2}$. The length of the crystal is adjusted to produce a gain of $10^{2}$ at the signal center wavelength: (a) $\lambda_{s}=850 \mathrm{~nm}$, (b) $\lambda_{s}=900 \mathrm{~nm}$, (c) $\lambda_{s}=550 \mathrm{~nm}$.

\subsection{OPA temperature bandwidth}

Temperature dependence of the refractive index can be successfully applied in noncritical phase matching, as described is section 2.4. An important design consideration in OPCPA systems that will produce high average power is the temperature bandwidth of OPA. The origin of the temperature bandwidth is in the temperatureinduced wave vector mismatch. The temperature bandwidth of common nonlinear materials used as OPAs is shown in Figs. 3.8-3.9. 
The advantage of $\mathrm{BBO}$ in high average power applications is evident from Figs.

3.8-3.9, indicating that over $30 \mathrm{~K} \mathrm{~cm}$ FWHM temperature bandwidth is available when operating near degeneracy.
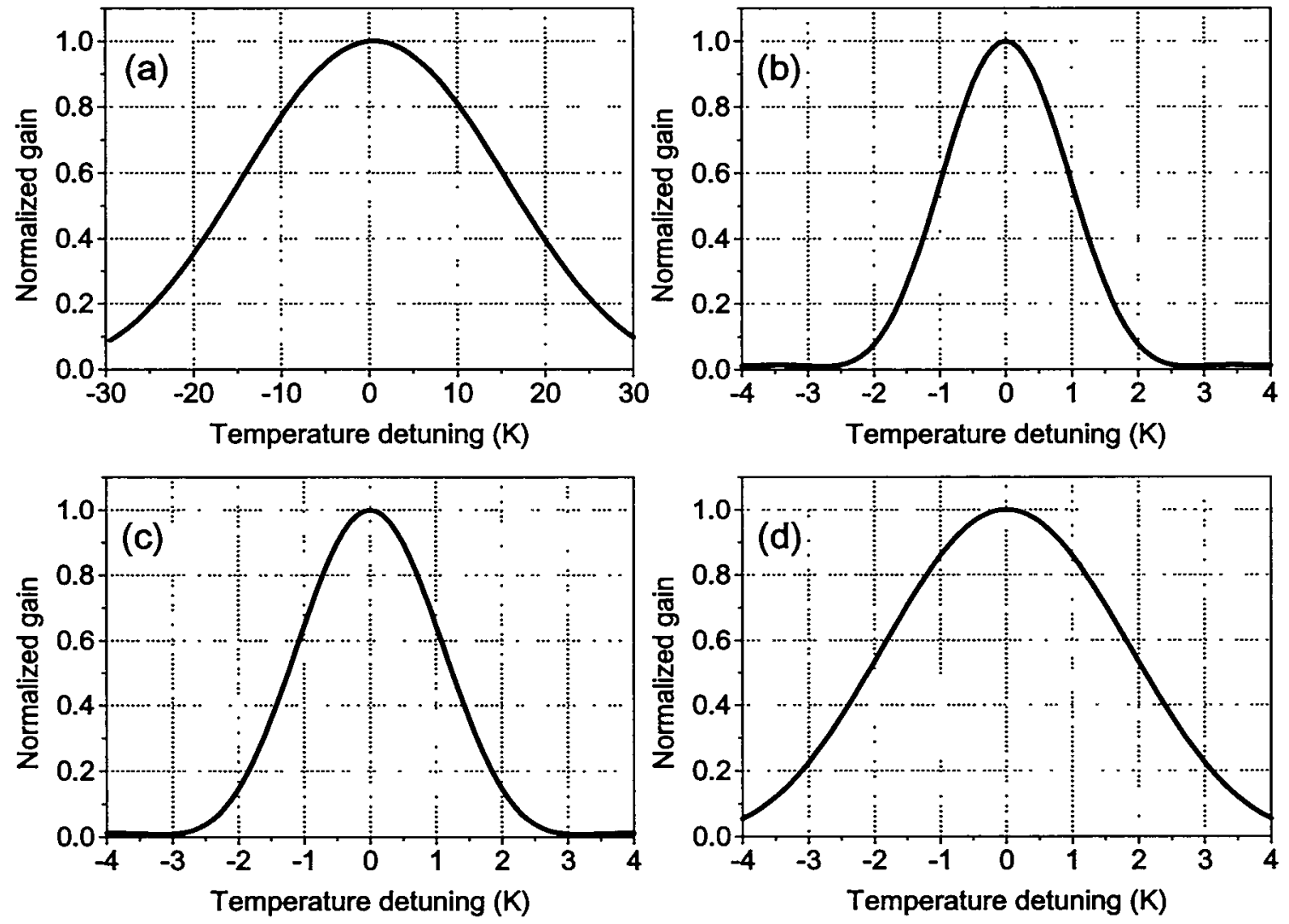

Figure 3.8. Nearly degenerate OPA temperature bandwidth for (a) BBO, (b) KDP, (c) critically phase-matched LBO, and (d) NCPM LBO. The crystals are pumped by $\lambda_{p}=532 \mathrm{~nm}$ at an intensity of $500 \mathrm{MW} / \mathrm{cm}^{2}$. The length of the crystal is adjusted to produce a gain of $10^{2}$ at the signal center wavelength $\lambda_{s}=1054 \mathrm{~nm}$. Center temperature is $294 \mathrm{~K}$, except in case (d), where it is $435 \mathrm{~K}$. 

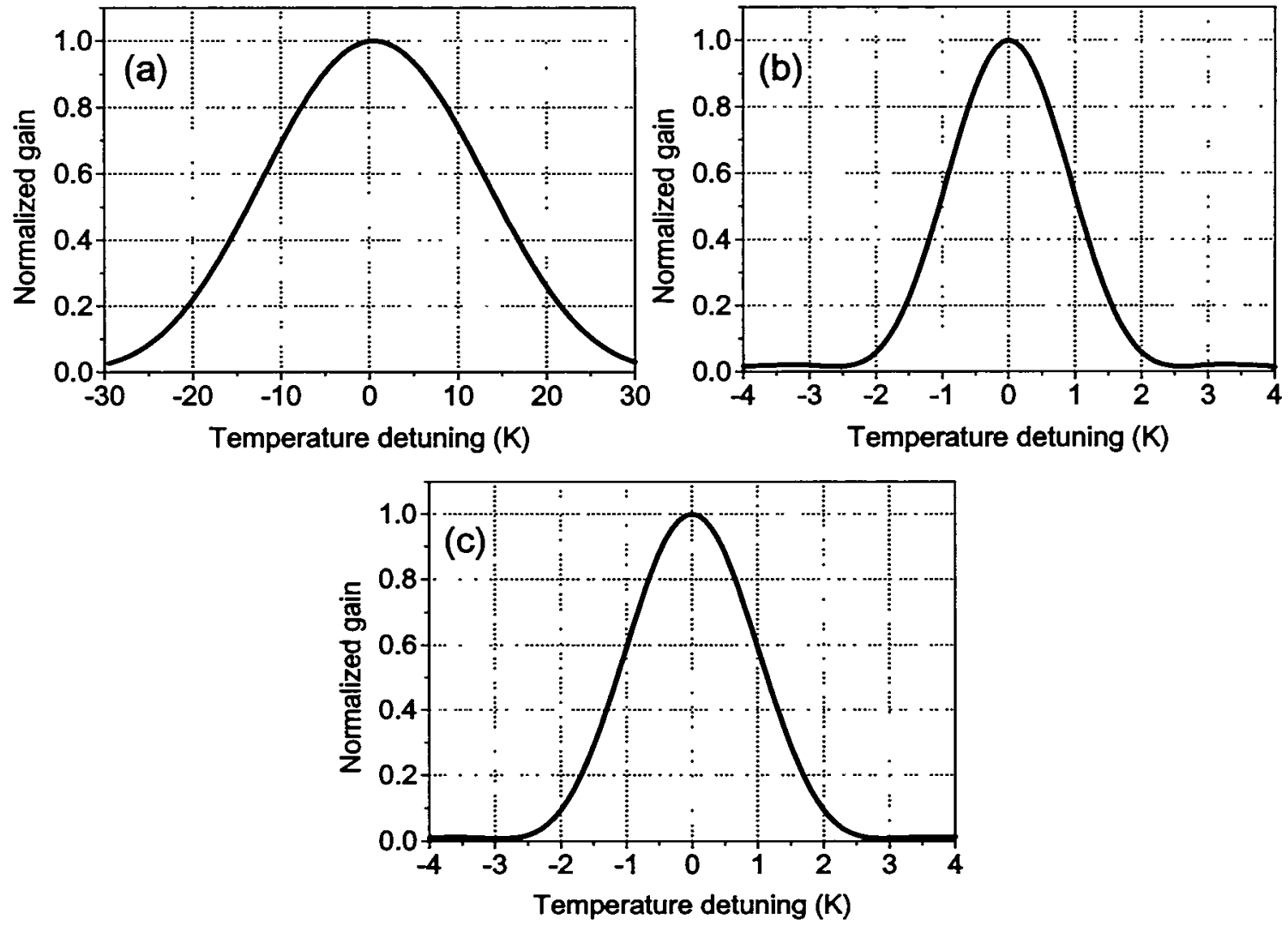

Figure 3.9 Non-degenerate OPA temperature bandwidth for (a) BBO, (b) KDP, and (c) critically phase-matched LBO. The crystals in (a) and (c) are pumped by $\lambda_{p}=532 \mathrm{~nm}$, while the crystal in case (b) is pumped by $\lambda_{p}=351 \mathrm{~nm}$. Pump intensity is $500 \mathrm{MW} / \mathrm{cm}^{2}$. The length of the crystal is adjusted to produce a gain of $10^{2}$ at the signal center wavelength: (a) $\lambda_{s}=800 \mathrm{~nm}$, (b) $\lambda_{s}=550 \mathrm{~nm}$, (c) $\lambda_{s}=800 \mathrm{~nm}$. Nonlinear angles are identical to the ones in Fig. 3.7. Center temperature is $293 \mathrm{~K}$.

\subsection{OPA angular bandwidth}

OPAs exhibit angular bandwidth associated with the deterioration of phase matching when the propagation direction of one or multiple beams deviates from the ideally phase-matched direction. This is particularly important in the case of deteriorating beam quality, when the beam propagates in an ensemble of directions. Two main effects 
contribute to dephasing with angular beam deviation. First, modification of the refractive index with the propagation direction modifies the magnitude (length) of the wave vectors. Second, modified noncollinear geometry contributes to dephasing through differences in the projection of the wave vectors in the direction perpendicular to the medium boundary.

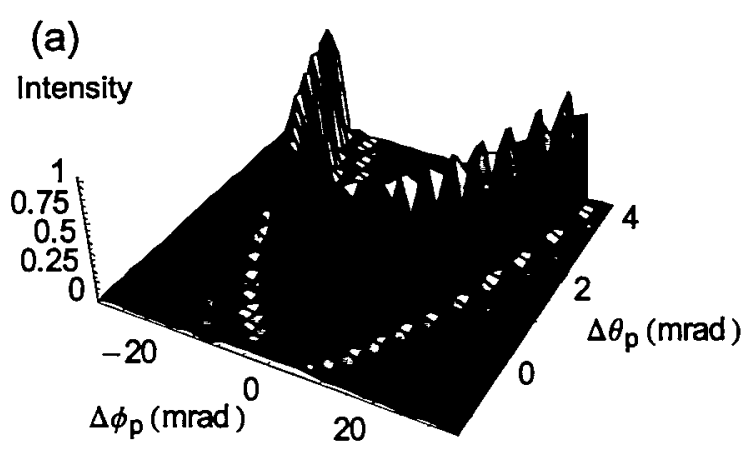

(c)

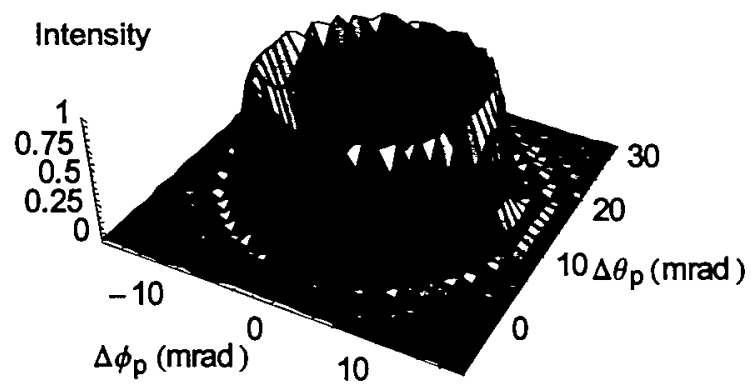

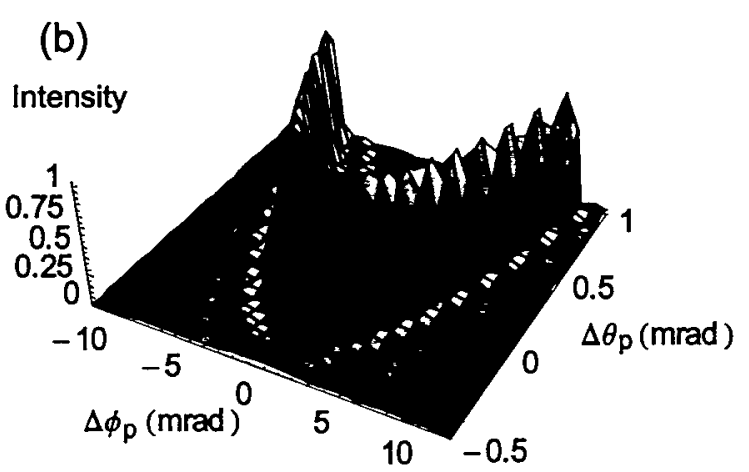

(d)

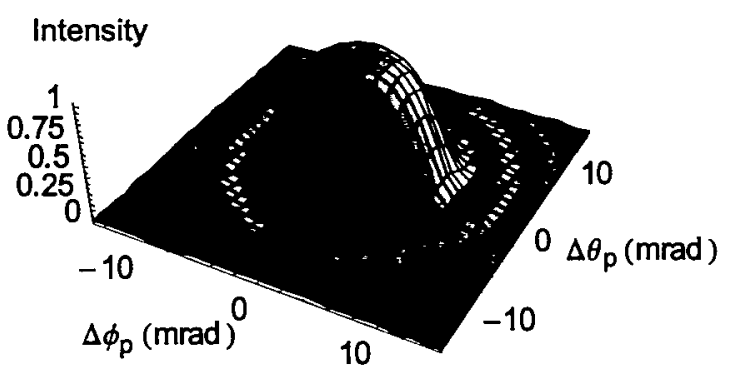

Figure 3.10. Angular bandwidth of (a) BBO, (b) KDP, (c) critically phase-matched LBO, and (d) NCPM LBO. The sensitivity is plotted as a function of angular deviation of the pump beam in two principal directions $(\theta$ and $\phi)$. The crystals are pumped by $\lambda_{p}=532 \mathrm{~nm}$ at an intensity of $500 \mathrm{MW} / \mathrm{cm}^{2}$. The length of the crystal is adjusted to produce a gain of $10^{2}$ at the signal center wavelength $\lambda_{s}=1054 \mathrm{~nm}$. The geometry at zero deviation is collinear.

The angular bandwidth of OPA with respect to angular deviation of the pump is calculated by utilizing phase matching considerations on a boundary between vacuum and nonlinear medium, as described in section 2.5. Results are shown in Fig. 3.10 for 
several nonlinear crystals. Note the particularly large angular bandwidth of NCPM LBO, indicating its large tolerance to beam quality.
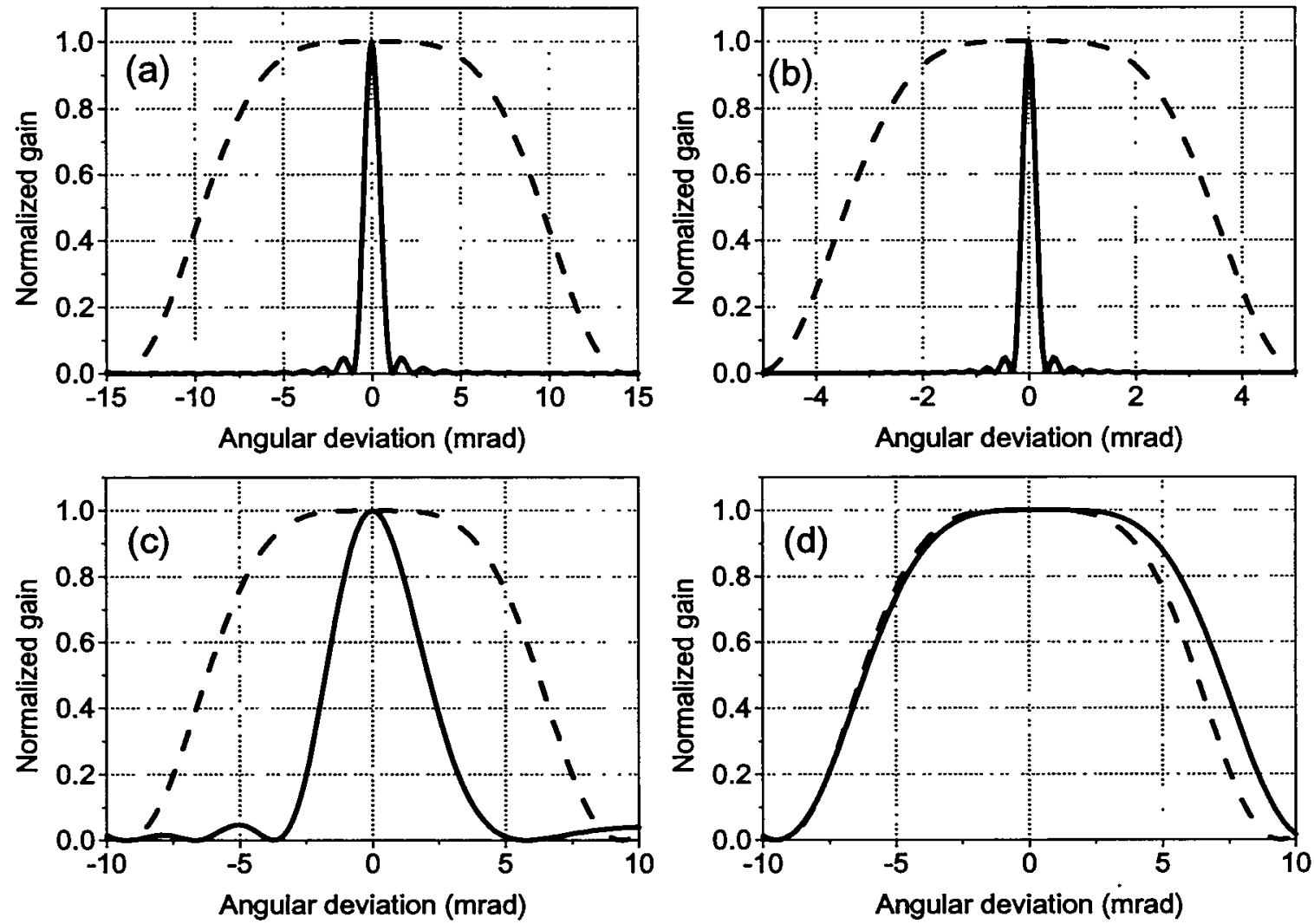

Figure 3.11. Angular bandwidth of (a) BBO, (b) KDP, (c) critically phase-matched LBO, and (d) NCPM LBO. The sensitivity is plotted as a function of angular deviation of the pump beam in one of the two principal directions, with zero deviation in the other direction. Solid lines represent dephasing in the sensitive direction $(\theta)$, while dashed lines represent dephasing in the insensitive direction $(\phi)$. The crystals are pumped by $\lambda_{p}=532 \mathrm{~nm}$ at an intensity of $500 \mathrm{MW} / \mathrm{cm}^{2}$. The length of the crystal is adjusted to produce a gain of $10^{2}$ at the signal center wavelength $\lambda_{s}=1054 \mathrm{~nm}$. The geometry at zero deviation is collinear.

Another feature of critically phase-matched crystals can be noted from Figs. 3.11: for extraordinarily polarized beams, a large anisotropy is observed in the angular tolerance. This is the result of a much stronger dependence of the wave vector mismatch on the change of amplitude of the refractive index than on the change in the wave vector 
projection perpendicular to the crystal surface. It is therefore very important to achieve a good beam quality and beam collimation in the crystal "sensitive" direction. These results are expanded in Chapter 8 , when attention is drawn to beam quality requirements in OPAs.

\subsection{Pulse contrast in OPA}

One difficulty with some applications in which high peak power laser systems are used is the requirement for high contrast level. In high-intensity target experiments, the prepulse associated with poor contrast level can cause a perturbation of the target before the arrival of the main laser pulse. The contributing factors to pulse contrast include amplified spontaneous emission (ASE) $)^{52}$, leakage from regenerative amplifiers and hardedge spectral clipping on the compressor gratings and other optical components. In the regenerative (multipass) amplification process, each time the pulse completes one roundtrip in the multipass cavity, a small fraction $(\sim 1 \%)$ leaks out of the cavity. Hence, the system produces prepulses spaced at integer multiples of cavity round-trip time ahead of the main pulse. While using larger compressor gratings can eliminate spectral clipping, the pulse contrast remains fundamentally limited by the ASE and leakage from regenerative amplifiers.

OPAs offer a way to improve the contrast level. Since optical parametric amplification is typically realized in a single-pass configuration, the prepulse due to the regenerative amplifier leakage is automatically eliminated. It is still important to evaluate the parametric fluorescence $(\mathrm{PF})^{53}$ in an OPA, a process analogous to ASE in lasers. 
While a full description of PF requires quantum mechanics, ${ }^{53,54}$ it is sufficient to consider the process as semiclassical. In that picture, $\mathrm{PF}$ is treated as optical parametric amplification of one photon per mode, arising from the vacuum field fluctuation. The "seed" photon can be in either the signal or the idler field, but the same result is obtained if $1 / 2$ of one photon is contained in each of the two fields. By considering the total number of modes, the angular and spectral power distribution can be calculated for PF.

For a simple calculation of PF, only the modes collinear and phase-matched over the entire bandwidth are considered. ${ }^{4}$ The seed power for $\mathrm{PF}$ is

$$
P_{\text {seed }}=h v \Delta v \text {, }
$$

where $h$ is the Planck's constant, $v$ is the center frequency, and $\Delta v$ is the frequency bandwidth over which phase matching occurs. The total PF amplified power can be simply calculated as ${ }^{13}$

$$
P_{P F}=P_{\text {seed }} G_{0},
$$

where $G_{0}$ is the small signal gain in OPA. This simple calculation can be used to compare PF to ASE from laser. Ross et $a l .^{13}$ performed preliminary calculations that indicate an improvement of one order of magnitude in the background PF compared to the ASE emanating from an equivalent Ti:sapphire laser.

The improvement of the contrast level in a system that uses OPCPA is more evident when the instantaneous nature of the amplification process is considered. Matching the pulse width of the signal and pump pulse in OPA guarantees that the amplification occurs only when the main signal pulse is present in the amplifier. This is in sharp contrast to laser amplifiers, which provide same or greater gain for prepulses than for the main pulse. 


\subsection{Spatial and temporal discretization}

In the experiments described in Chapters $4-6$, numerical modeling was found to be a critical step for optimizing the performance of OPCPA systems. The developed OPCPA model is based on a numerical solution for the system of coupled differential equations for difference-frequency generation. The system that describes traveling waves can be written in a concise form as derived in section 2.2:

$$
\begin{gathered}
\frac{d A_{1}}{d z}=i \frac{2 \omega_{1}}{n_{1} c} d_{e f f} A_{2}^{*} A_{3} \exp (i \Delta k z), \\
\frac{d A_{2}}{d z}=i \frac{2 \omega_{2}}{n_{2} c} d_{e f f} A_{1}^{*} A_{3} \exp (i \Delta k z), \\
\frac{d A_{3}}{d z}=i \frac{2 \omega_{3}}{n_{3} c} d_{e f f} A_{1} A_{2} \exp (-i \Delta k z) .
\end{gathered}
$$

where $A_{1}, A_{2}$ and $A_{3}$ are the amplitudes of the electric field of the signal, idler and pump, respectively, $d_{e f f}$ is effective nonlinearity, and $\Delta k$ is the wave vector mismatch. The temporal walk-off due to group velocity dispersion is neglected because only long (nanosecond) pulses are used. Diffraction effects are not included in the model explicitly due to their weak relative impact compared with the temporal and spatial modulation. In particular, two effects arising from diffraction can influence gain and conversion efficiency in optical parametric amplification. First, variable beam diameter causes axially nonuniform intensity. Second, angular dephasing leads to reduced gain due to finite angular acceptance of the nonlinear process. Both of these effects are insignificant with weakly focused beams used in nanosecond OPCPA. 
Eqs. (3.2) model the interaction of plane waves and have to be suitably modified to describe the situation when the beam diameter is finite and the beam has a nonuniform transverse intensity profile. In addition, temporal and spectral modification of the signal pulse occurs in OPCPA when the pump pulse intensity is not a temporal constant. Finally, the effect of Poynting vector walk-off for extraordinary waves in nonlinear crystals presents an additional effect that requires inclusion in the model. This is particularly important when the beam diameter is comparable to the total transverse walkoff over the nonlinear crystal length.

Those effects can be included by solving Eqs. (3.2) numerically on a 3-dimensional grid in space and time. The assumed intensity distribution at a point is:

$$
I_{i}(x, y, t)=2 n_{i} \sqrt{\frac{\varepsilon_{0}}{\mu_{0}}}\left|A_{i}(x, y, t)\right|^{2}, \quad i=1,2,3,
$$

where $I$ is the beam intensity, $x$ and $y$ are the two transverse coordinates, and $t$ is the temporal coordinate. The intensity distribution is normalized in the following fashion:

$$
\int_{-\infty-\infty}^{\infty} \int_{-\infty}^{\infty} \int_{i}(x, y, t) d x d y d t=E_{i}, \quad i=1,2,3
$$

where $E$ is the pulse energy. A zero phase is assigned to the electric field of the seed and pump at the input face of the crystal $(z=0)$. This is consistent with the insensitivity of optical parametric amplification with respect to the initial phase relationship between the seed and the pump when an idler is not initially present. Additionally, the effect of pump beam Poynting vector and noncollinear angle is introduced. Poynting vector walk-off angle, $\rho$, is assumed in one direction $(x)$, and the noncollinear angle for the signal $\left(\Omega_{1 x}, \Omega_{1 y}\right)$ and idler beam $\left(\Omega_{2 x}, \Omega_{2 y}\right)$ is assumed in two directions. The appropriate 
transverse parts the signal, idler and pump are accounted for at the point $z$ in the crystal by calculating the corrected transverse displacement:

$$
\begin{gathered}
x_{1}^{\prime}=x_{1}+\Omega_{1 x} z, y_{1}^{\prime}=y_{1}+\Omega_{1 y} z, \\
x_{2}^{\prime}=x_{2}+\Omega_{2 x} z, y_{2}^{\prime}=y_{2}+\Omega_{2 y} z, \\
x_{3}{ }^{\prime}=x_{3}+\rho z,
\end{gathered}
$$

where $\left(x_{1}^{\prime}, y_{1}^{\prime}\right),\left(x_{2}^{\prime}, y_{2}^{\prime}\right)$ and $x_{3}^{\prime}$ represent the corrected transverse coordinates for the signal, idler and pump, respectively.

In CPA the spectrum is linearly chirped in time, so the phase mismatch due to spectral bandwidth can be included by assigning the appropriate value for wave vector mismatch and its projection in the direction normal to the crystal surface:

$$
\begin{gathered}
\mathbf{k}(t)=\mathbf{k}_{3}-\mathbf{k}_{1}(t)-\mathbf{k}_{2}(t), \\
\Delta k_{z}(t) \approx 2 \pi\left(\frac{n_{3}}{\lambda_{3}}-\frac{n_{1}(t)}{\lambda_{1}(t)}-\frac{n_{2}(t)}{\lambda_{2}(t)}\right)
\end{gathered}
$$

Finally, the system of differential equations can be written as

$$
\begin{aligned}
& \frac{d A_{1}\left(x_{1}{ }^{\prime}, y_{1}{ }^{\prime}, t\right)}{d z}=i \frac{2 \omega_{1}(t)}{n_{1}(t) c} d_{e f f} A_{2}^{*}\left(x_{2}{ }^{\prime}, y_{2}{ }^{\prime}, t\right) A_{3}\left(x_{3}{ }^{\prime}, y, t\right) \exp (i \Delta k(t) z) \\
& \frac{d A_{2}\left(x_{2}{ }^{\prime}, y_{2}{ }^{\prime}, t\right)}{d z}=i \frac{2 \omega_{2}(t)}{n_{2}(t) c} d_{e f f} A_{1}^{*}\left(x_{1}{ }^{\prime}, y_{1}{ }^{\prime}, t\right) A_{3}\left(x_{3}{ }^{\prime}, y, t\right) \exp (i \Delta k(t) z) \\
& \frac{d A_{3}\left(x_{3}{ }^{\prime}, y, t\right)}{d z}=i \frac{2 \omega_{3}(t)}{n_{3}(t) c} d_{e f f} A_{1}\left(x_{1}{ }^{\prime}, y_{1}{ }^{\prime}, t\right) A_{2}\left(x_{2}{ }^{\prime}, y_{2}{ }^{\prime}, t\right) \exp (-i \Delta k(t) z)
\end{aligned}
$$

A discretization of the electric field is performed on the grid of $(D X, D Y, D T)$ points with a spatial and temporal window size $(W X, W Y, W T)$, such that the following normalization applies: 


$$
2 n_{i} \sqrt{\frac{\varepsilon_{0}}{\mu_{0}}} \frac{W X}{D X} \frac{W Y}{D Y} \frac{W T}{D T} \sum_{j=1}^{D X} \sum_{k=1}^{D Y} \sum_{l=1}^{D T} \mid A_{i}(j, k, l)^{2}=E_{i}, \quad i=1,2,3
$$

Fig. 3.12 illustrates the main features introduced in the numerical model. The system (3.11) in discrete form is solved numerically using a 4th order Runge-Kutta integration routine, yielding solutions for electric field amplitude and phase at the crystal output in a spatially and temporally resolved form. It is important to note that the model allows initial misalignment of the seed and pump beam at the input face of the nonlinear crystal. This is a frequent experimental optimization procedure required to achieve best overlap of the beams inside a crystal which exhibits walk-off for one of the beams.

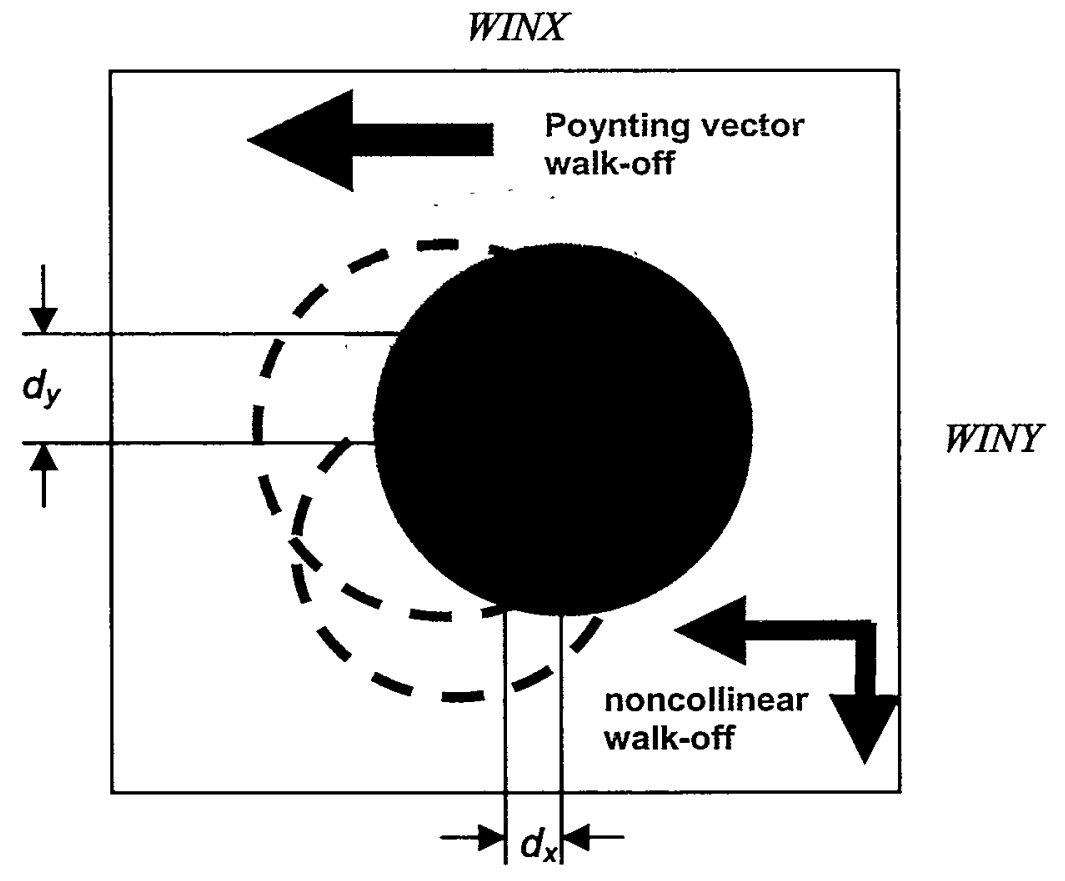

Figure 3.12. Transverse interaction of the signal (red) and pump (green) beam in the numerical model. Indicated is the direction of transverse walk-off due to Poynting vector and noncolinearity. 


\section{Chapter 4}

\section{Optical parametric amplifier as a preamplifier}

\section{for high-energy Nd:glass lasers}

In this Chapter, a high pulse energy femtosecond pulse source based on OPCPA is presented. $1 \mu \mathrm{m}$ broadband oscillator pulses were successfully amplified to $31 \mathrm{~mJ}$ and recompressed to $310 \mathrm{fs}$ pulse duration, at a $10 \mathrm{~Hz}$ repetition rate. The gain in the system is $6 \times 10^{7}$, achieved by single passing only $40 \mathrm{~mm}$ of gain material pumped by a tabletop commercial Q-switched Nd:YAG laser. This relatively simple system replaces a more complex Ti:sapphire regenerative amplifier-based CPA system. Numerous features in design and performance of optical parametric chirped pulse amplification make it a preferred alternative to regenerative amplification based on Ti:sapphire in the front end of high peak power lasers.

In section 4.1 , the advantages of the new broadband amplification technology are established, followed by a detailed description of the system design. A novel design for a high-gain preamplifier is presented as a part of the system. Experimental results follow in sections 4.3 and 4.4. The obtained results presented in this section represent a 50 -fold improvement in extracted energy and a 10-fold improvement in conversion efficiency when compared with the previous OPCPA designs utilizing similar tabletop pump lasers. 


\subsection{Motivation}

CPA is now routinely used to generate gigawatt, terawatt, and even petawatt ${ }^{8}$ peak powers for applications such as short-pulse precision machining, ${ }^{55}$ high-harmonic generation and time resolved spectroscopy,${ }^{56}$ and fast ignitor driver for inertial confinement fusion, ${ }^{57}$ respectively. Bandwidth limitations of high-energy laser gain media can be partially circumvented by using high-bandwidth materials such as Ti:sapphire in the system front end, combined with high-energy storage materials such as Nd:glass in the final amplifiers (see example in Fig. 4.1).

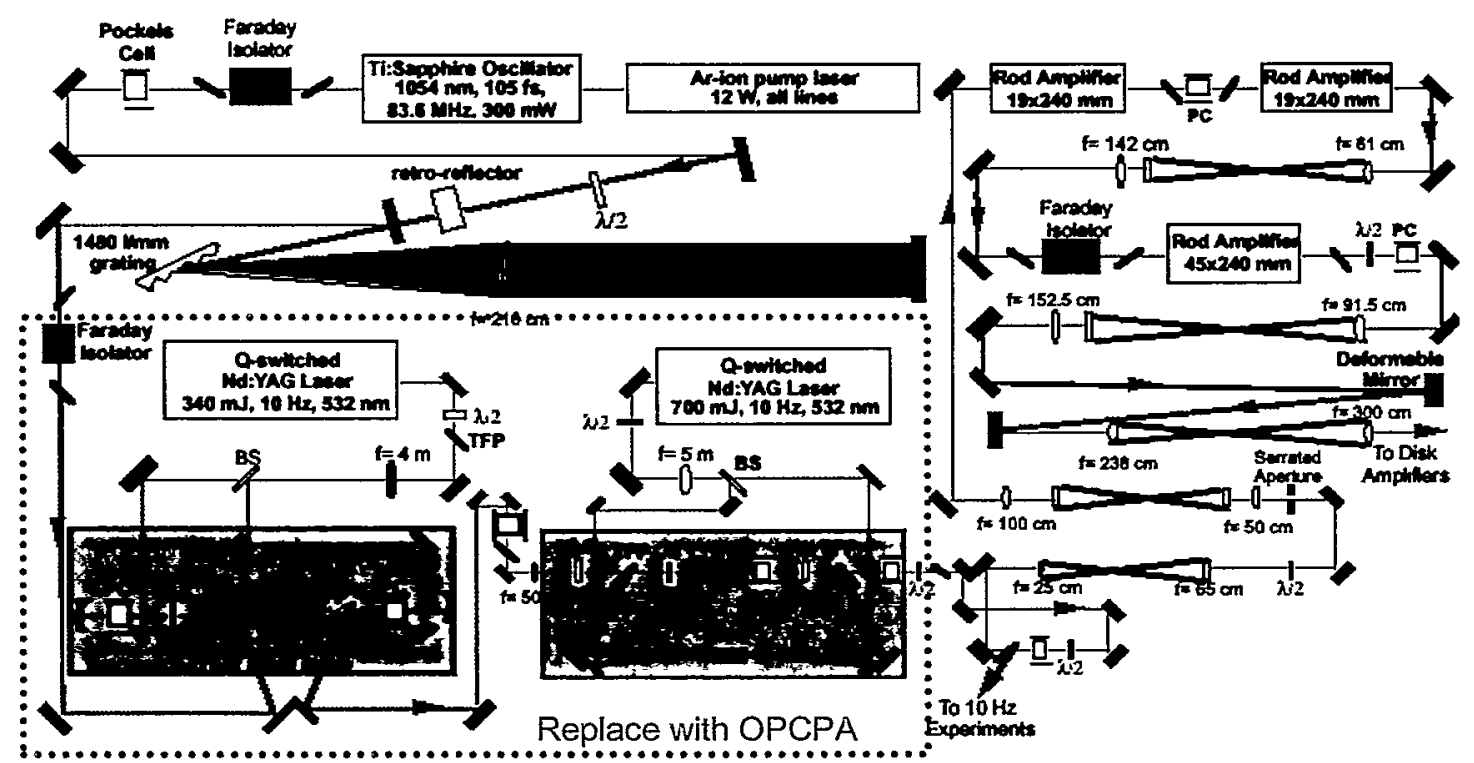

Figure 4.1. Design of the Petawatt laser ${ }^{44}$ front end at the Lawrence Livermore National Laboratory. Indicated are two pump lasers and two Ti:sapphire regenerative amplifiers that are replaced by an OPCPA system.

Different applications require different combinations of pulse energy and pulse width: spectroscopic applications require $\mu \mathrm{J}$ to $\mathrm{mJ}$ pulse energies with $65-100$ fs pulse widths, machining applications require pulse energies in the range of $0.1-50 \mathrm{~mJ}$ with pulse 
widths between $0.5-2 \mathrm{ps}$, and fast ignitor drivers require relatively large pulse energies (10$100 \mathrm{~kJ})$ with fairly long pulse widths (1-10 ps).

The existing technologies for short pulse generation and amplification rely upon laser hosts such as Cr:LiSAF, Ti:sapphire, and Yb:YAG. Yb:YAG is used where direct diode pumping of the source is desired. Otherwise, green-pumped Ti:sapphire is utilized. Nd:glass amplifiers find application when large pulse energies with relatively long pulse widths (hundreds of fs) are sought. OPCPA has been recently identified as an attractive technology for broadband amplification. An underappreciated feature of optical parametric amplification is that it results in a minimal heat deposition in the OPA crystal, allowing a different set of material constraints to limit the final energy. Combined with the demonstrated performance of existing doubled pump lasers, OPCPA appears capable of extending CPA into the kilowatt average power regime. ${ }^{58}$

OPCPA has the same broad bandwidth capability as Ti:sapphire, but offers the flexibility of operating at an arbitrary wavelength, making OPAs more flexible than solid state lasers. High gain can be obtained from nanosecond OPCPA by single passing a relatively short length $($ several $\mathrm{cm}$ ) of gain material, which eliminates the need for complicated regenerative multipass amplification and electro-optic switching. Since the total length of the gain material is short, the accumulated nonlinear phase (B-integral) is substantially reduced, enabling good amplified pulse recompression..$^{51}$ A substantial reduction of the prepulse from a CPA system can be achieved by eliminating the pulse leakage originating from the regenerative multipass cavity. In addition to the mentioned advantages of OPCPA when used to replace regenerative amplifiers, OPCPA also allows large energy scaling through large available apertures of potassium dihydrogen phosphate 
(KDP) crystals.

Another feature of an OPA makes it even more attractive for applications where the reduction of pulse aberrations is crucial. Optical parametric amplification is inherently a low-noise amplification process, allowing possible pump phase aberrations to be transferred to the idler wave, while introducing a negligible modulation to the signal wave. The only noise that is introduced in the process is due to parametric fluorescence ${ }^{53,54}$ process analogous to ASE in lasers, and is typically less intense than ASE for similar gain. ${ }^{13}$ The condition for signal amplification to occur with high fidelity is a good overlap between the signal and the idler waves in space and time. A reduced transverse beam overlap and pump beam nonuniformity lead to spatial modulation of the signal, while a reduced temporal overlap can produce a modified chirped pulse spectrum.

\subsection{System design}

The design of the OPCPA system is presented schematically in Fig. 4.2, while Fig. 4.3 shows the OPCPA in operation. A mode-locked Ti:sapphire oscillator (Spectra-Physics Tsunami) with a center wavelength of $1054 \mathrm{~nm}$ produces $100 \mathrm{fs}$ transform-limited pulses. The pulse train consists of $2.5 \mathrm{~nJ}$ pulses at $84 \mathrm{MHz}$. A single pulse is selected from the oscillator pulse train using a Pockels cell and a pair of polarizers. The pulse is stretched in a four-pass stretcher which contains a 1480 lines/mm diffraction grating and a plano-convex lens, with the effective grating separation of $8 \mathrm{~m}$. The stretcher is configured to clip the spectrum at its FWHM, with the bandwidth of $16.5 \mathrm{~nm}$. The stretched pulse width is $3 \mathrm{~ns}$, and the stretched pulse energy is $0.5 \mathrm{~nJ}$. As noted in section 4.4, spherical and chromatic 
aberrations in the stretcher limit the recompression of the seed pulse to $280 \mathrm{fs}$.

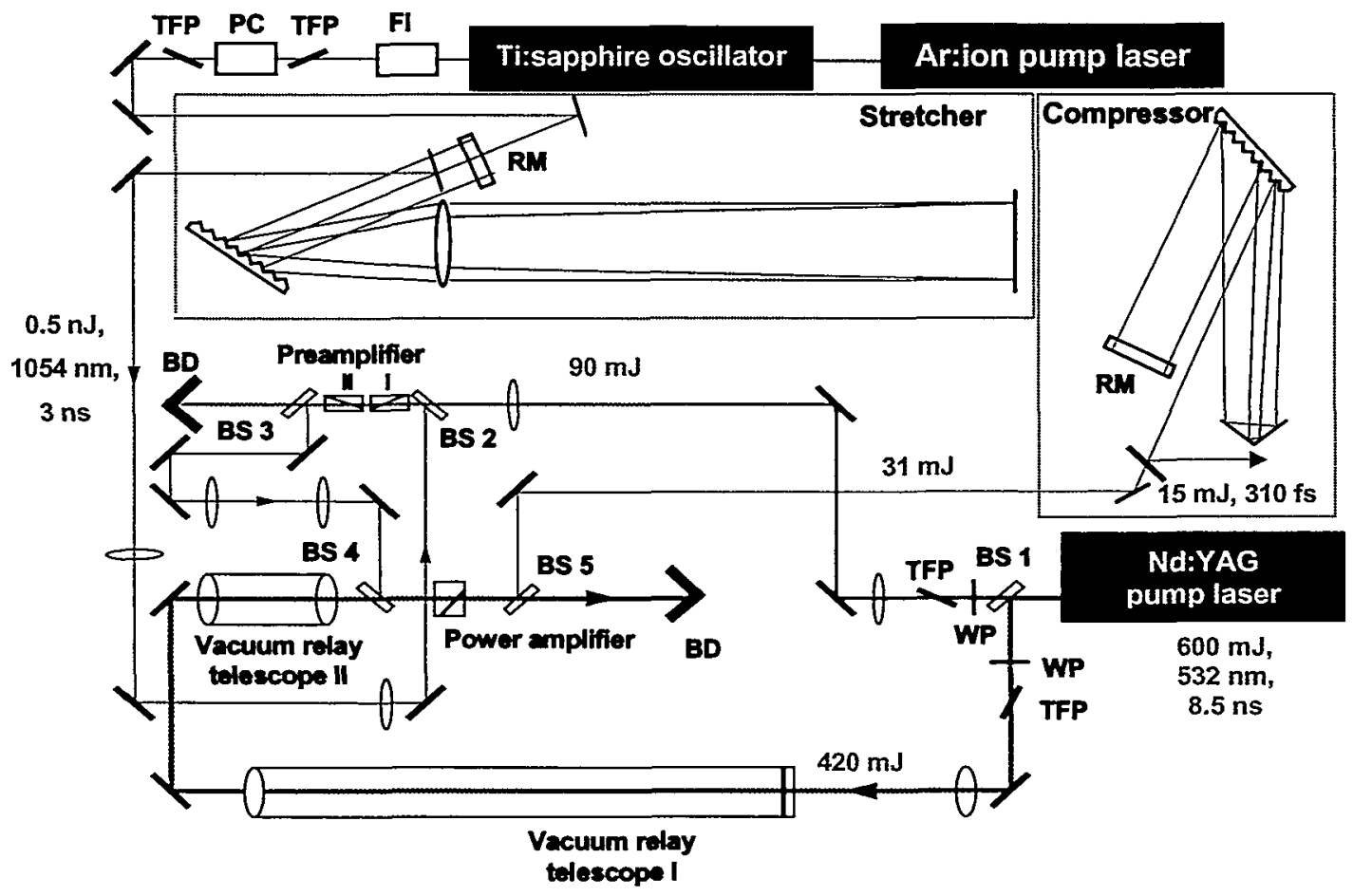

Figure 4.2. Experimental OPCPA setup for replacing Ti:sapphire regenerative amplifiers. BS-beamsplitter, TFP-thin film polarizer, WP-waveplate, BD-beam dump, FI-Faraday isolator, PC-Pockels cell, RM-roof mirror.

The stretched seed pulse is then relay imaged to the OPA. The OPA consist of three $\beta$-barium borate (BBO) crystals. The size of the first two crystals is $4 \times 4 \times 15 \mathrm{~mm}^{3}$, while the third crystal is $10 \times 10 \times 10 \mathrm{~mm}^{3}$, permitting scaling to large incident pump pulse energy by increasing the pump beam diameter. The crystals are cut at $22.8^{\circ}$ to allow type I angular phase matching, and they have a $2^{\circ}$ wedge on their output faces to prevent intra-crystal parasitic oscillation. Without the wedge on the crystals, parasitic oscillation would inevitably manifest itself due to a very large gain in a single OPA crystal. The result would be too stringent requirements on the crystal antireflection coatings. 


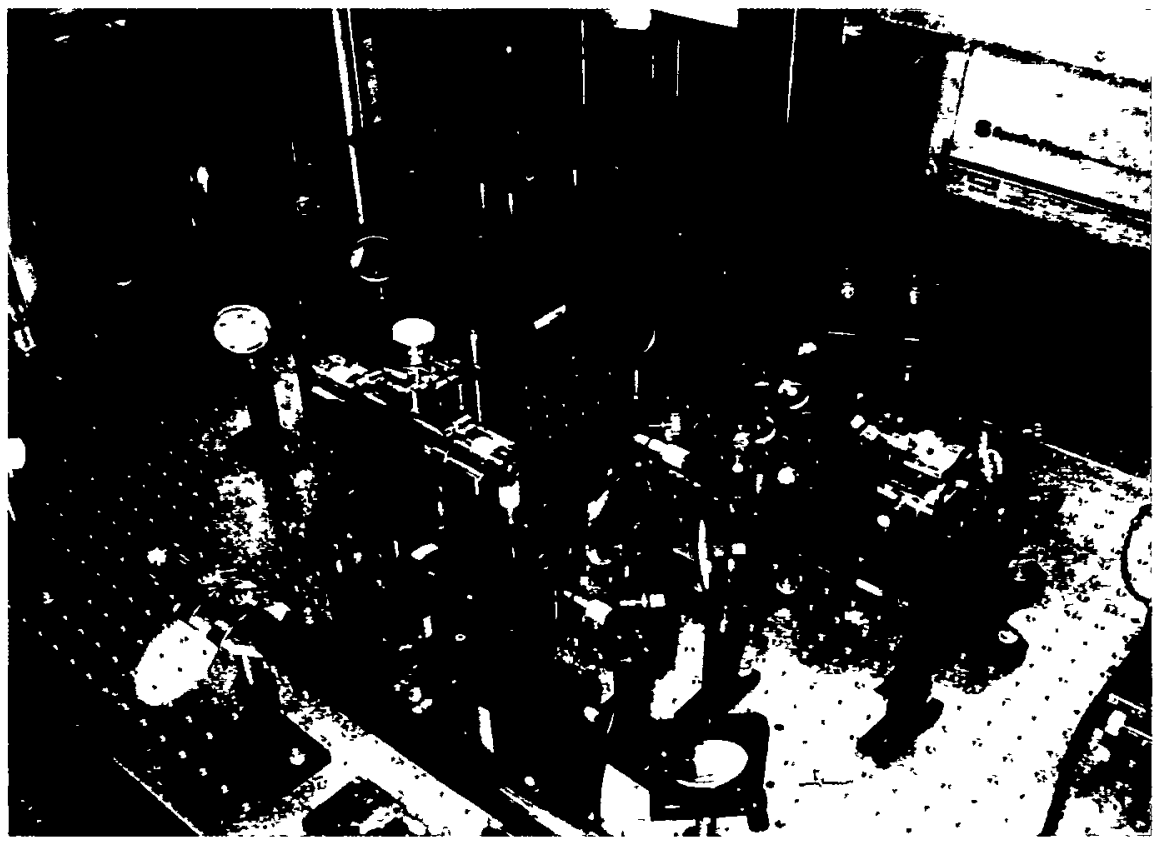

Figure 4.3. OPA during operation, producing $31 \mathrm{~mJ}$ pulses at a 10 $\mathrm{Hz}$ repetition rate
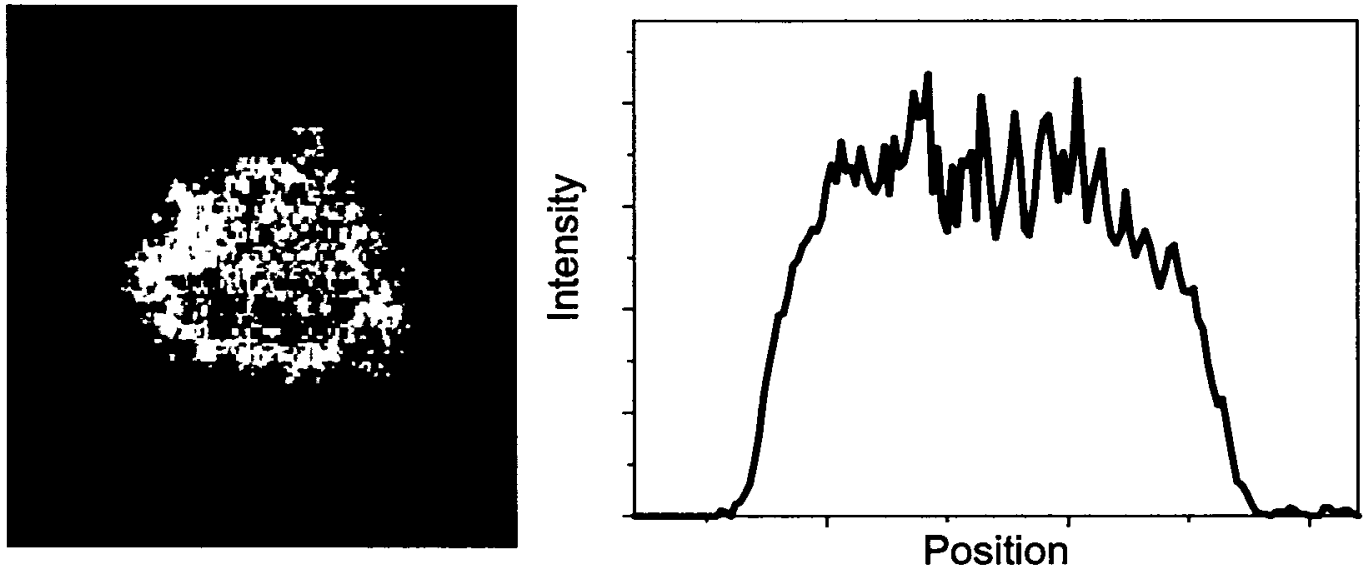

Figure 4.4. Pump beam near field intensity profile is nearly supergaussian

The pump source for the OPA is a Spectra-Physics GCR 270-10 Nd:YAG laser, operated in a single longitudinal mode, producing $600 \mathrm{~mJ}$ of $532 \mathrm{~nm}$ output. The pulse width of Q-switched pulses is $8.5 \mathrm{~ns}$, at a $10 \mathrm{~Hz}$ repetition rate. The transverse intensity 
profile of the pump beam is a supergaussian (Fig. 4.4), while the temporal profile is gaussian, with a characteristic pulse spatiotemporal evolution for an unstable resonator. ${ }^{59}$ Spatially resolved temporal evolution of the pump pulse was measured first. For this purpose, the pump laser beam at the OPA crystal was suitably magnified and relay imaged onto a $400 \mu \mathrm{m}$ pinhole, and a fast silicon diode detector was centered behind the pinhole. Amplitude, pulse width and build-up delay was recorded as a function of radial position of the pinhole and the numerical fit to the results is shown in Fig. 4.5. A significant build-up delay and difference in pulse width exists for different radial positions in the pump beam, as a result of pulse build-up in an unstable resonator in the pump laser. While the center of the pump pulse has a slowly variable build-up delay as a function of radial position when compared to the position in the wings of the pump beam, its pulse width is longest at the beam center.
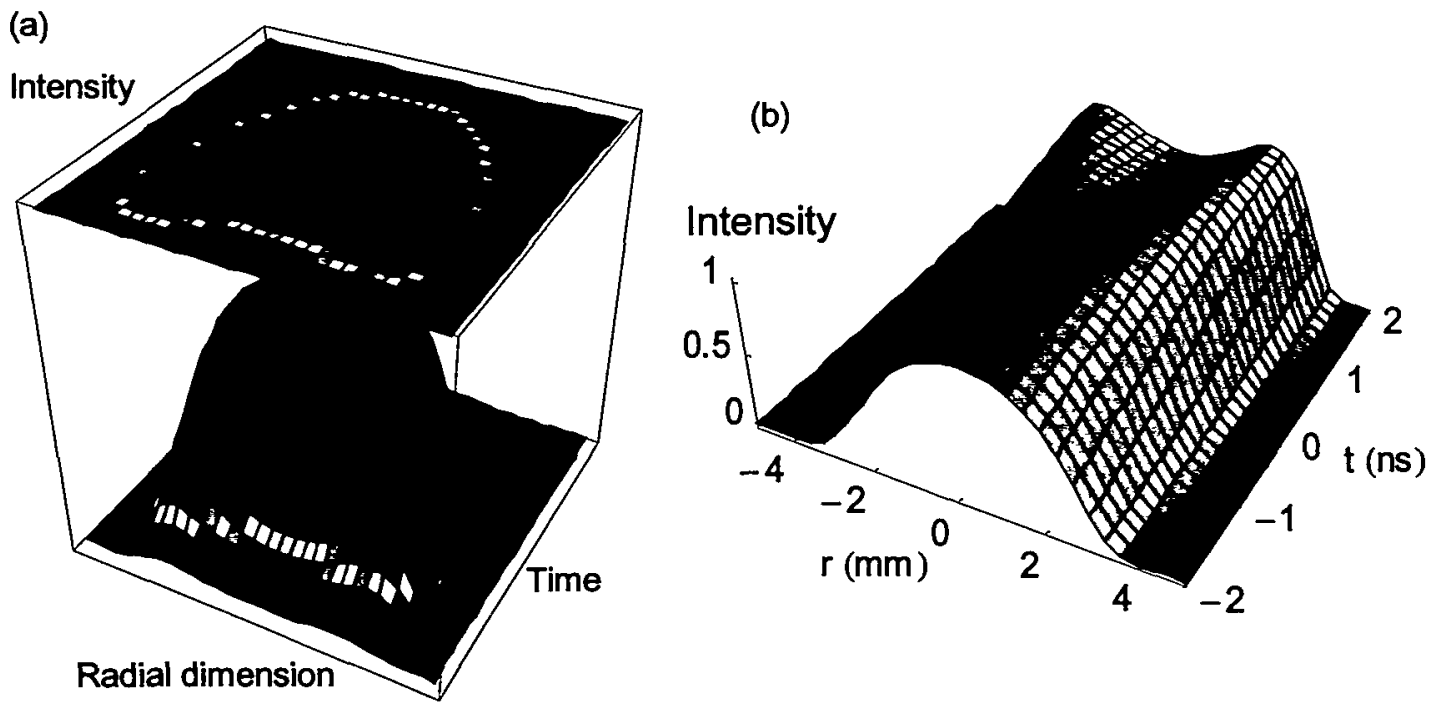

Figure 4.5. Spatiotemporal evolution of the pump pulse originating in an unstable laser resonator. Shown is the normalized intensity as a function of the radial position $r$ and time $t$, (a) for entire pulse, and (b) in a 4 ns temporal window. 
This is a particular difficulty for use in optical parametric amplification, which requires high beam intensities and shorter pulses. Additionally, the problem of Poynting vector walk-off is anticipated to be more critical in OPA pumped by this pulse compared to a pulse which has no radial distribution of build-up delay. This is the result of the instantaneous effective beam size in interaction smaller than the temporally integrated beam size, giving rise to reduced overlap between the signal and the pump beam.

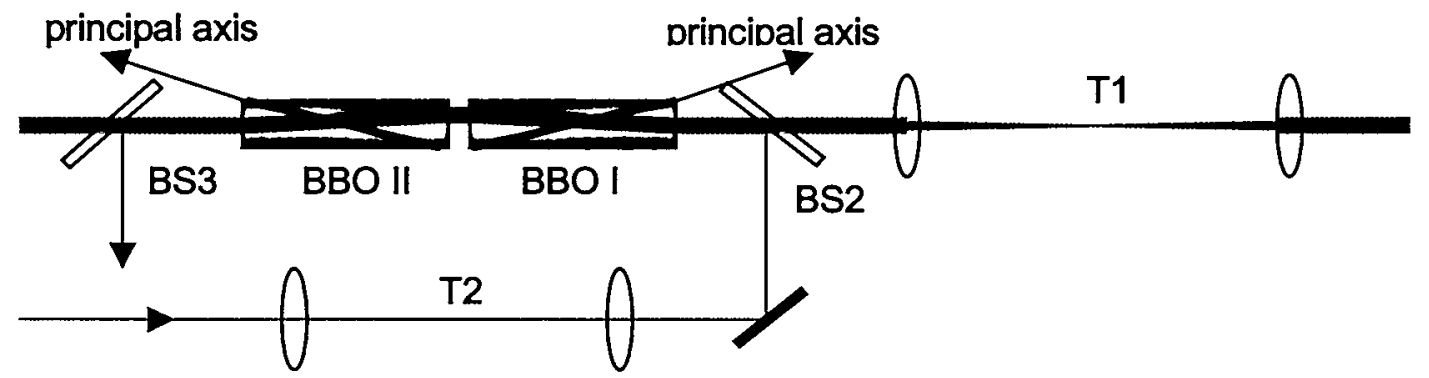

Figure 4.6. High-gain preamplifier design. Two BBO crystals are arranged for walkoff compensation. The reduction of the gap between the crystals minimizes dephasing in air and allows imaging using only a single pair of telescopes $\mathrm{T} 1$ and $\mathrm{T} 2$. The signal is injected into the preamplifier using beamsplitter BS2, and it is separated from the pump beam using beamsplitter BS3.

The first two BBO crystals are configured as a high-gain preamplifier (Figs. 4.6 and 4.7) and separated by $2 \mathrm{~mm}$. Walk-off compensation ${ }^{60}$ is used in order to reduce the effect of the intrinsic $3.2^{\circ}$ extraordinary beam Poynting vector walk-off in BBO. The pump beam is split by a $15 \%$ beamsplitter BS1 into a $90 \mathrm{~mJ}$ beam and a $510 \mathrm{~mJ}$ beam. The supergaussian transverse intensity profile of the pump laser is relay imaged between the first two BBO crystals by means of a telescope, which simultaneously adjusts the pump beam diameter in the first two crystals to $1.6 \mathrm{~mm}$. The proximity of the two walk-off compensated crystals in the preamplifier eliminates the need for separate imaging of the 
pump beam on each crystal, which is a significant simplification when compared to previous designs. ${ }^{61,62}$ The peak intensity of the pump beam in the first and second BBO crystal is $450 \mathrm{MW} / \mathrm{cm}^{2}$. The pump beam is collimated in order to minimize dephasing due to the small angular acceptance of BBO $(0.4 \mathrm{mrad} \mathrm{cm})$.

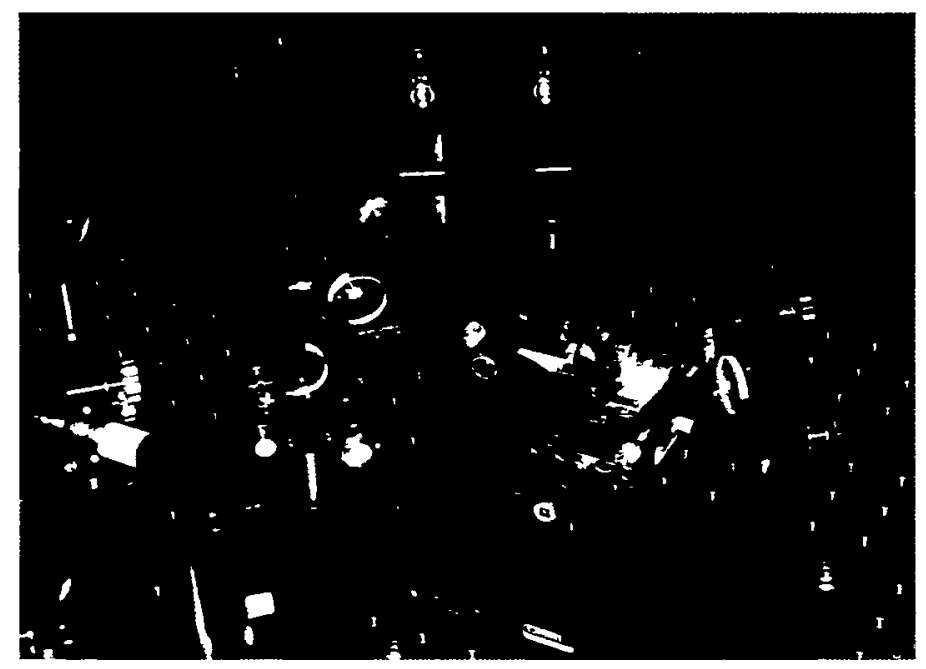

Figure 4.7. High-gain preamplifier during operation. The design simplicity is achieved by combining walk-off compensation with a close proximity of two BBO crystals.

An additional source of dephasing is the beam quality from the pump source (we measured the pump beam quality to be $1.6 \mathrm{xDL}$ in the sensitive phase matching plane and $1.8 \times \mathrm{xL}$ in the insensitive phase matching plane). The measured deviation of the pump beam from ideal beam quality has negligible impact on parametric gain with the selected pump beam diameter (see Chapter 8).

The size of the seed beam is dictated by the requirement to avoid spatial modulation on the signal beam due to transverse walk-off of the extraordinary polarized pump beam, and is set to $0.4 \mathrm{~mm}$ in the first two $\mathrm{BBO}$ crystals. The center of the seed beam is displaced from the center of the pump beam by $0.4 \mathrm{~mm}$ in the sensitive phase matching direction on 
the front face of the first $\mathrm{BBO}$ crystal, maximizing the spatial overlap of the beams in the preamplifier. The first two crystals are treated as large-gain preamplifiers and the low conversion efficiency resulting from the small spatiotemporal overlap of the seed and the pump pulses is neglected. The seed beam is introduced into the crystal at a $1^{\circ}$ external angle with respect to the pump beam, perpendicular to the crystal principal plane. The amplified signal and the idler beam are spatially separated after propagating $30 \mathrm{~cm}$ out of the preamplifier, and the idler is subsequently blocked, leaving only the amplified signal.

The supergaussian spatial profile of the pump beam transmitted through the $15 \%$ beamsplitter BS1 is relay imaged onto the final BBO crystal (power amplifier). The pump imaging telescope consists of two vacuum image relays. The use of two vacuum relay telescopes in the system is necessary because of spatial constraints and the requirement for imaging, beam sizing and temporal matching of the seed and pump between the preamplifier and the power amplifier. The pump beam diameter in the power amplifier is $3.5 \mathrm{~mm}$, with peak intensity of $430 \mathrm{MW} / \mathrm{cm}^{2}$ on the crystal with $410 \mathrm{~mJ}$ incident pump energy. Operating at an intensity near $0.5 \mathrm{GW} / \mathrm{cm}^{2}$ is a compromise between the requirement for strong nonlinear drive and the reduction of the risk of crystal damage, leading to greater system reliability. At operating intensities near $1 \mathrm{GW} / \mathrm{cm}^{2}$, crystal damage was periodically observed, which is unacceptable in a system with appreciable repetition rate. The signal beam is imaged from the preamplifier and introduced into the power amplifier using the beamsplitter BS4. The signal and idler beams are separated spatially after 2 meters of propagation out of the power amplifier crystal, given the larger diameter of the signal and idler beam in the power amplifier.

The amplified signal pulse is compressed using a single-grating, double-pass 
compressor. The compressor contains a 1480 lines/mm grating, set at a diffracted angle of $55.33^{\circ}$ to match the stretcher angle. No spectral clipping occurs in the compressor as a result of the large size of the associated optics. The spectral range is limited by the hard spectral clip in the stretcher, which limits the size of the spectrally dispersed beam in the compressor. The measured compressor efficiency is $50 \%$, as a result of the degradation of the again surface gold layer. The recompressed pulse width measurement is performed using a scanning intensity autocorrelator for the recompressed seed, and a single-shot intensity autocorrelator for the amplified signal pulse at $10 \mathrm{~Hz}$.

\subsection{Experimental results - preamplifier}

Single-crystal experiments were performed first, using $15 \mathrm{~mm}$ and $10 \mathrm{~mm}$ long BBO crystals. With the injected signal of $0.5 \mathrm{~nJ}$, the measured gain from the $14.5 \mathrm{~mm}$ uncoated BBO crystal was 2400 , while the maximum gain of the $10 \mathrm{~mm}$ BBO crystal was 180 . Gain was optimized by adjusting the transverse displacement between the seed and the pump on the front face of the first preamplifier crystal. Single pass gain was recorded as a function of pump intensity, and the experimental results are shown in Fig. 4.7, along with the result of the numerical code described in section 3.7. Excellent agreement was observed between the calculation and the experiment. The only adjustable parameter in the code is the displacement between the two beams on the crystal front face, which corresponds to the experimental gain optimization.

When the uncoated $14.5 \mathrm{~mm}$ long crystal was replaced with a $15 \mathrm{~mm}$ long coated crystal, the maximum gain obtained was 3700 . This crystal was finally used as the first 
crystal in the OPCPA preamplifier (BBO I), while the $14.5 \mathrm{~mm}$ uncoated crystal was used as BBO II. From the second BBO crystal $1.5 \mathrm{~mJ}$ was obtained (Gain=830).
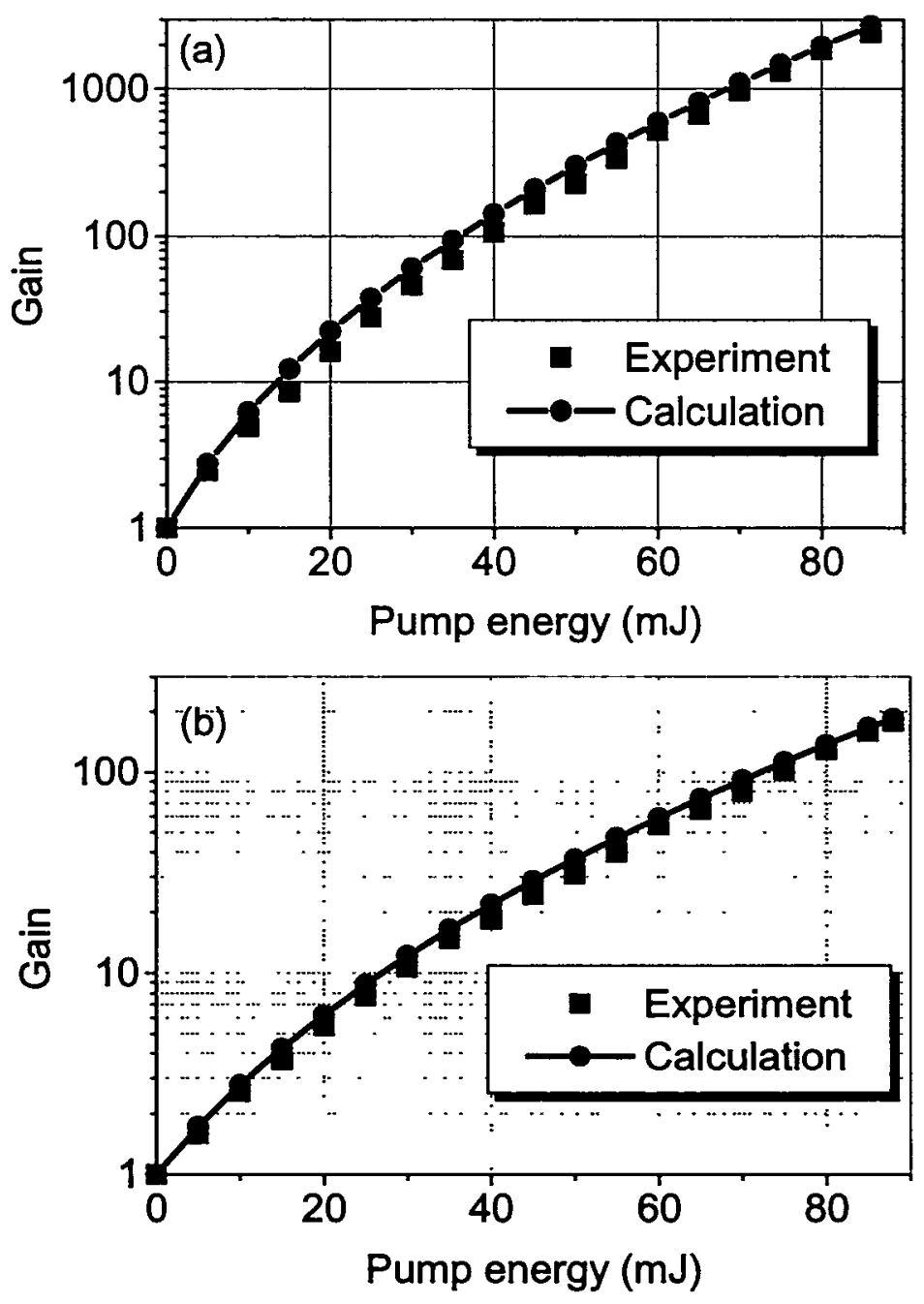

Figure 4.8. Experimental and theoretical results for single-pass gain from (a) $14.5 \mathrm{~mm}$ long, and (b) $10 \mathrm{~mm}$ long BBO crystal.

The measured amplified signal pulse width, after passing through the preamplifier, was $3 \mathrm{~ns}$ at the clip points in the temporal domain. This is identical to the original stretched pulse width at its clip points in the temporal domain. Through adjustment of the waveplate, 
the amplified signal energy exiting the preamplifier was varied by changing the pump intensity.

Amplified transverse beam profiles in the near and far field for signal are shown in Fig. 4.9. Beam quality measurement was performed on the seed, amplified signal beam and the idler beam. The results are shown in Table 4.1, following the usual $\mathrm{M}^{2}$-notation described in Chapter 8.
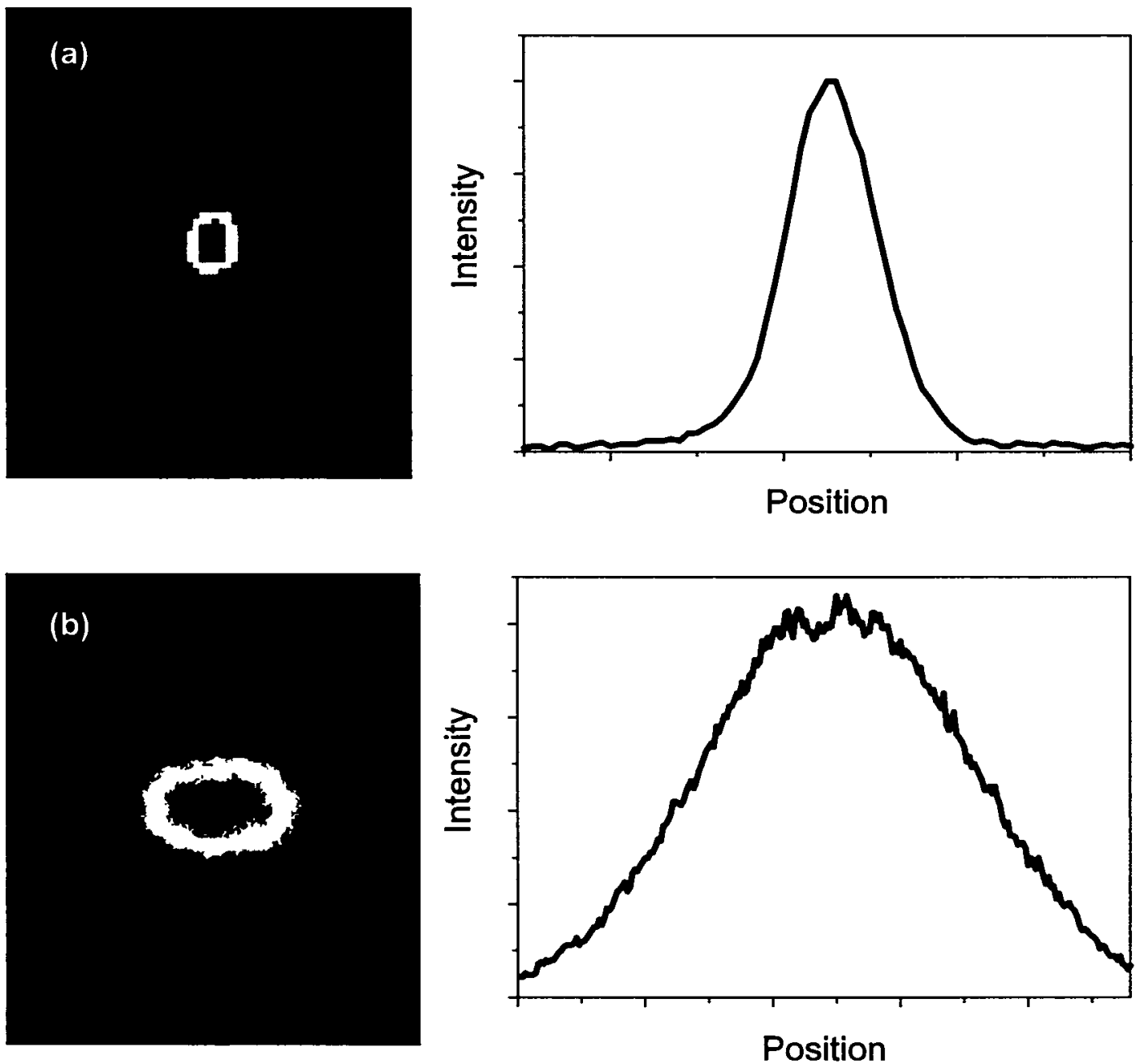

Figure 4.9 Signal intensity profile of the preamplifier, in (a) near field, and (b) far field. 


\begin{tabular}{|c|c|c|c|}
\hline & Seed & Signal & Idler \\
\hline $\mathrm{M}^{2}$ (horizontal) & 1.16 & 1.60 & 2.35 \\
\hline $\mathrm{M}^{2}$ (vertical) & 1.28 & 1.29 & 1.85 \\
\hline
\end{tabular}

Table 4.1. Beam quality from the preamplifier

The source of the measured imperfect beam quality of the seed is the hard beam clip that occurs on the stretcher lens. Optical parametric amplification is a process which conserves the signal beam quality to a very high degree. However, the small diameter of the pump beam in the preamplifier leads to a strong effect of pump beam walk-off in the crystal sensitive direction. It is believed that this spatial effect gives rise to the increase in the observed $M^{2}$ in the sensitive direction of the crystal.

\subsection{Experimental results - power amplifier and compression}

The expected small signal gain in the power amplifier is 160 . A maximum gain of 20 was measured in the power amplifier operating in the regime of strong pump depletion. The signal beam is amplified up to $31 \mathrm{~mJ}$, with $1.5 \mathrm{~mJ}$ of injected signal from the preamplifier. In Fig. 4.10 the extracted energy for several preamplifier outputs is shown. The maximum pump to signal conversion efficiency in the power amplifier crystal is achieved when the preamplifier output is set to $1.5 \mathrm{~mJ}$. The pump-to-signal conversion efficiency is $25 \%$, by including only the pump energy contained in the temporal overlap of the pump with the signal. The overall pump to signal conversion efficiency in this OPCPA system is $6 \%$. The 
numerical model was applied to calculate the energy extraction in the final amplifier. As shown in Fig. 4.10, fairly good agreement was observed between the numerical model and the experimental results. The only adjustable parameter in the numerical code was again the displacement of the signal and pump beam at the front face of the power amplifier crystal.

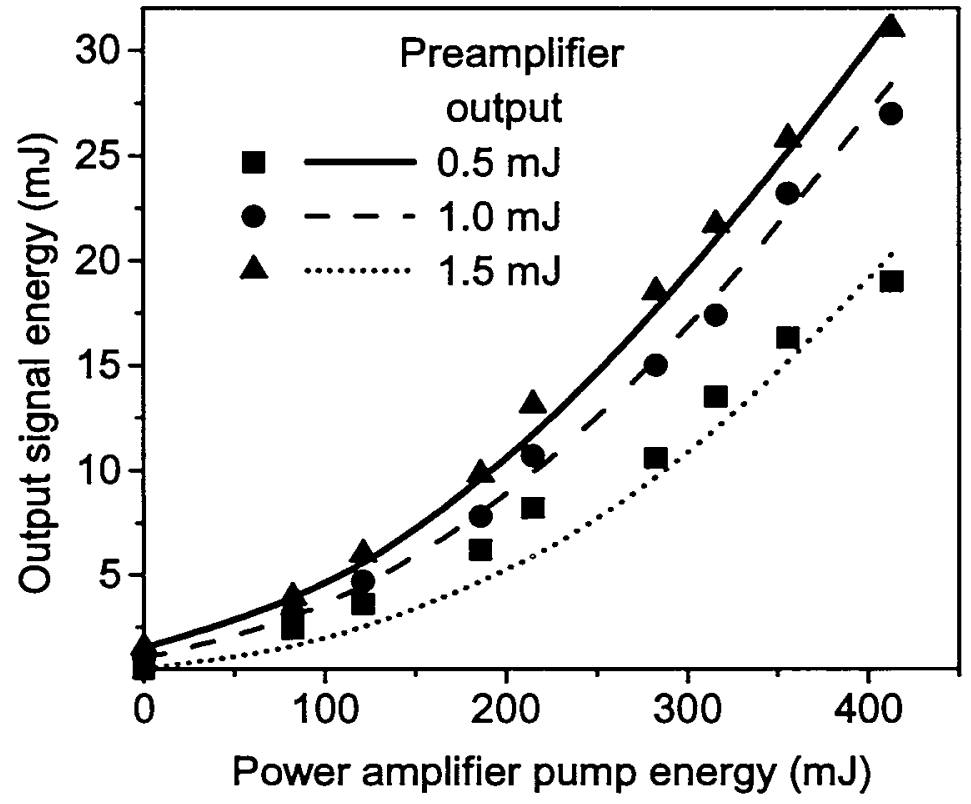

Figure 4.10. Experimental extraction data from the final BBO amplifier, for different preamplifier output energies. The results of the numerical model are shown in red.

Fig. 4.11 shows the measured spectra for stretched seed, amplified signal from the preamplifier and the amplified signal from the power amplifier. The measured bandwidth of the pulse is $16.5 \mathrm{~nm}$ at the spectral clip points in both the preamplifier and the power amplifier. This is consistent with the absence of bandwidth narrowing due to intrinsic OPA bandwidth. In this configuration the power amplifier of the OPA is operated in the depleted pump regime in order to obtain a large extracted energy. This is done at the expense of the 
recompressed pulse contrast level, which remains limited by the hard clip on the spectrum occurring in the pulse stretcher. The amplified signal spectral shape is modified by strong pump depletion in the power amplifier and resembles a top-hat shape. The measured output stability of the signal is greatly improved in the regime of strong pump depletion, ${ }^{63}$ reducing the effect of pump energy variation and temporal jitter.

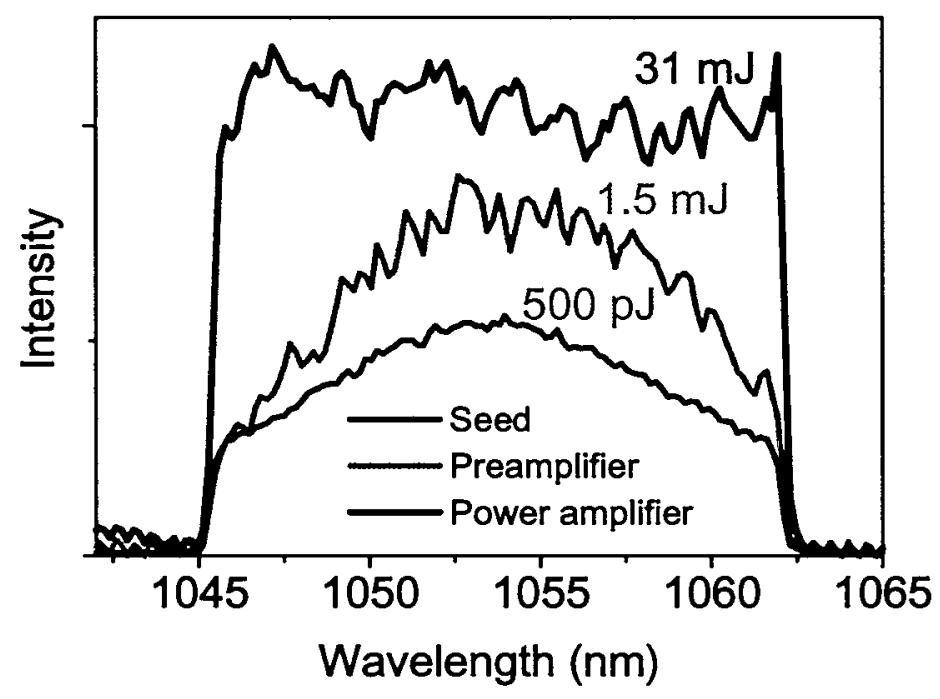

Figure 4.11. Seed and amplified signal spectra from preamplifier and power amplifier. Spectral modifications occur in preamplifier and power amplifier.

The depletion of the pump beam was measured by recording the total scattered pump light after the power amplifier. The relative time delay between the seed and pump is optimized prior to measurement to obtain maximum extraction. In Fig. 4.12, the experimentally measured depletion of the pump beam is evident as a dip which occurs $\sim 2.5$ ns prior to the peak power of the pump pulse. To explain the fact that the maximum extraction occurs prior to the point of maximum pump power, it is important to evaluate the spatially varying power, which is a result of the complex spatiotemporal evolution of the 
pump pulse. In Fig. 4.12, the measured evolution of the pump pulse is used to calculate the pump power of the entire beam, and of the central portion of the beam. The central portion of the beam is defined as the beam area which encloses $70 \%$ of the true beam $1 / \mathrm{e}^{2}$ diameter. The peak of the power of the central portion of the beam corresponds with the measured point of maximum pump depletion. Since the center of the pump beam is more relevant to amplification in OPCPA because of its good overlap with the signal, the maximum extraction is obtained at the point of the maximum power of the central portion of the beam.

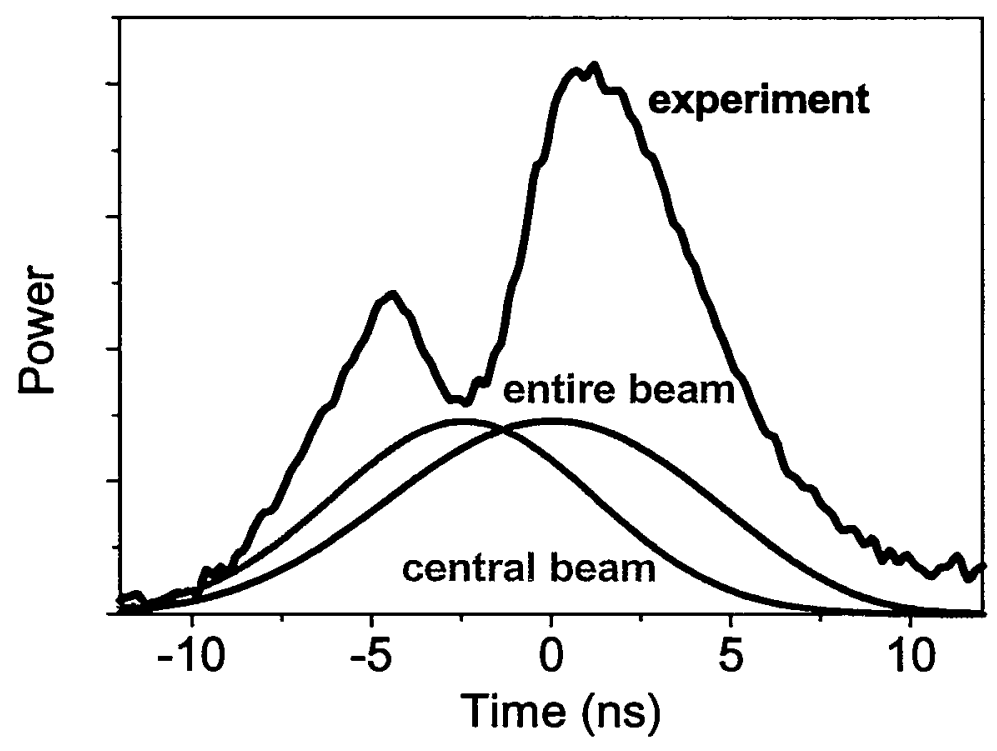

Figure 4.12. Instantaneous recorded pump power after OPCPA shows significant pump depletion. Indicated is the modeled instantaneous power for the entire beam, and the instantaneous power of the center of the beam ( $70 \%$ of the beam diameter).

The beam quality obtained from the power amplifier is shown in Table 4.2 , while the near-field and far-field transverse intensity profiles for amplified signal from the power amplifier are shown in Fig. 4.13. The beam quality of the signal is improved after 
amplification, which is the result of spatial filtering in OPA and a minimal effect of pump beam walk-off.

\begin{tabular}{|c|c|c|}
\hline & Seed & Signal \\
\hline$M^{2}$ (horizontal) & 1.6 & 1.03 \\
\hline$M^{2}$ (vertical) & 1.29 & 1.1 \\
\hline
\end{tabular}

Table 4.2 Beam quality from the power amplifier
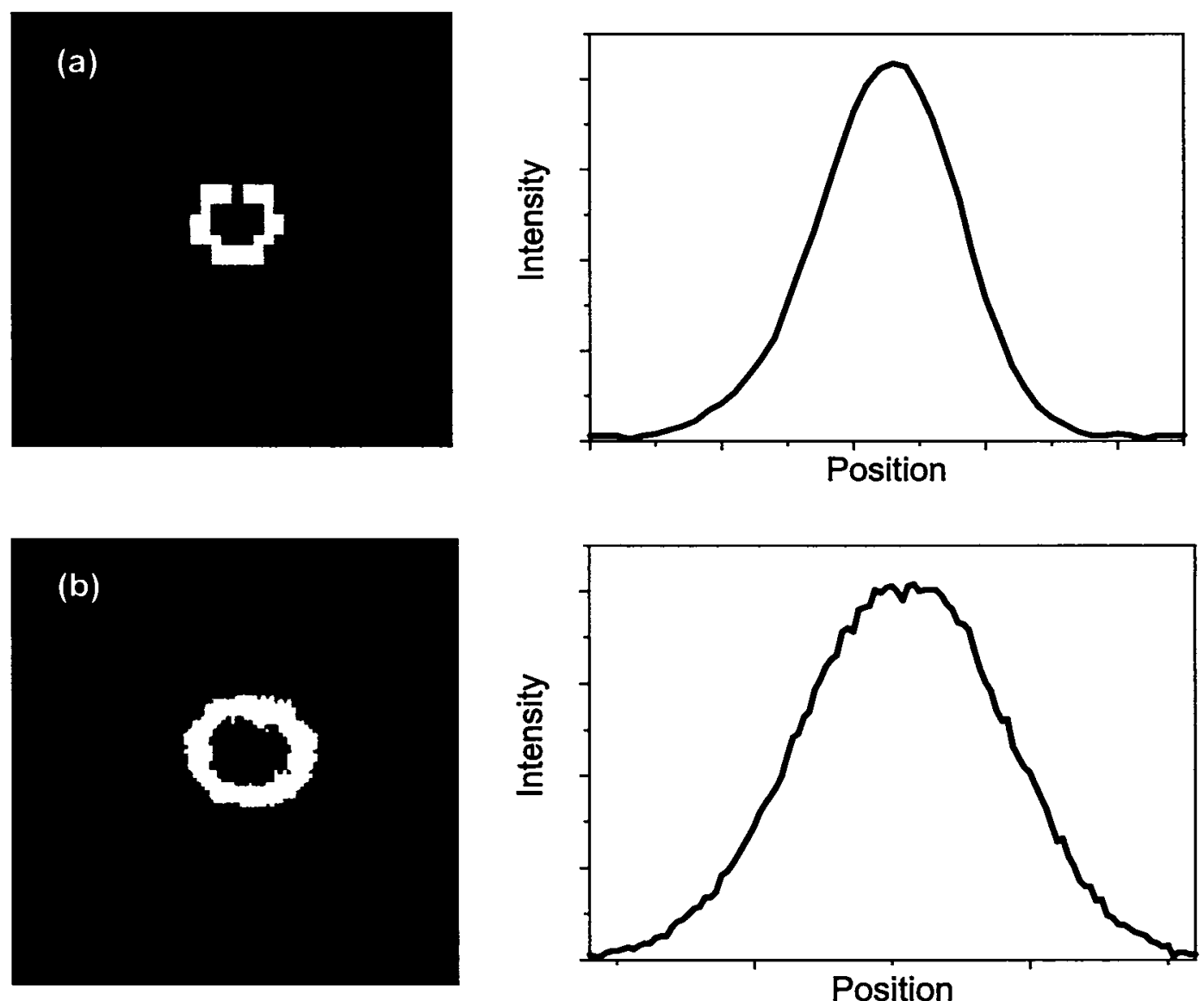

Figure 4.13. Signal intensity profile of the power amplifier in (a) near field, and (b) far field.

As mentioned previously, the stretched $0.5 \mathrm{~nJ}$ seed pulses were initially compressed 
to 280 fs FWHM (Fig. 4.14), limited by the spherical and chromatic aberrations occurring in the pulse stretcher, which contains a lens-based refractive telescope. ${ }^{64}$ The intensity autocorrelation of the recompressed pulse at an energy of $31 \mathrm{~mJ}$ is shown in Fig. 4.14 (b). The recompressed amplified pulse duration is measured to be 310 fs FWHM, a $10 \%$ increase compared to the best achieved seed recompression before amplification in OPA. The recompressed pulse pedestal is decreased after amplification. This is probably the result of the simultaneous presence of uncompensated phase terms from the stretchercompressor pair and the modification of amplified spectrum in OPCPA operating in the regime of strong pump depletion.
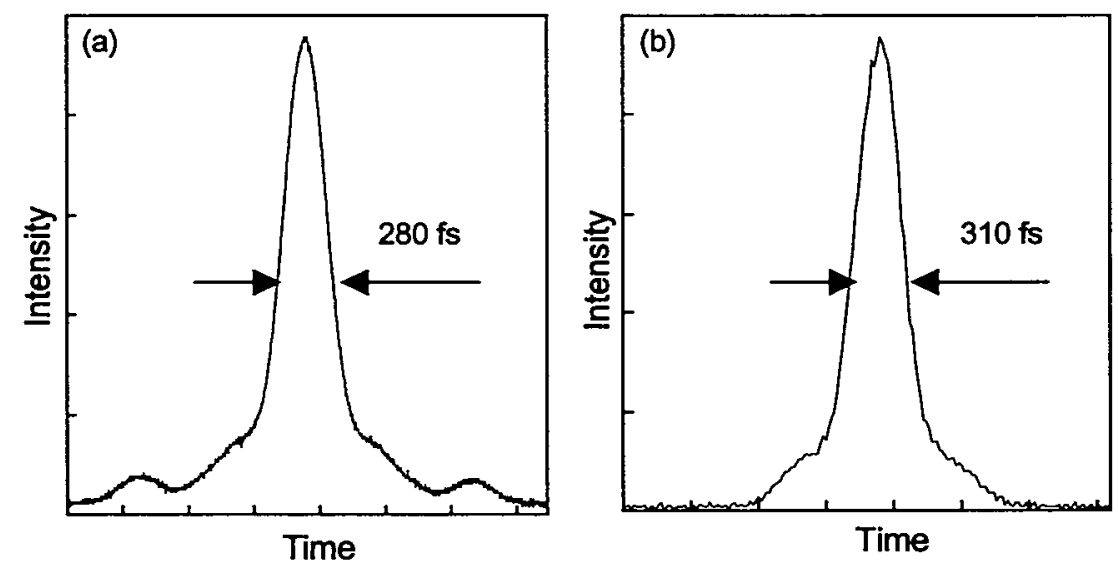

Figure 4.14. Recompressed pulse intensity autocorrelation for (a) seed pulses, and (b) amplified pulses. Deconvolved pulse width is (a) $280 \mathrm{fs}$ and (b) 310 fs.

\subsection{Conclusion}

In summary, a successful use of OPAs for broadband, high-fidelity CPA was demonstrated. The presented OPCPA system produced a 50-fold increase in amplified 
pulse energy and a 10-fold improvement in efficiency compared to previous systems pumped by tabletop commercial Q-switched pump lasers. ${ }^{61,62}$ The improved efficiency is a result of several factors. Asymmetric splitting of the pump pulse is utilized to obtain a high gain from the preamplifier with small fraction of the pump energy discarded. This is followed by the efficient power amplifier with a large pump beam and a short BBO crystal, resulting in good spatial overlap between the pump and the signal. Walk-off compensated double-crystal preamplifier design allows a relatively small pump beam to be used in the preamplifier. Pump beam is collimated to reduce the angular dephasing in the type I process in BBO. The increased stretched seed pulse width allows a greater fraction of the pump energy to be transferred to signal. The conversion efficiency in the temporal window defined by the stretched seed pulse compares with the result of the OPCPA experiment performed with a large glass laser as a pump. ${ }^{65}$

The wavelength and pulse energy level from the demonstrated OPCPA is ideal for seeding high-energy Nd:glass amplifiers of high-power lasers. The peak power of $50 \mathrm{GW}$ was obtained from a relatively compact and simple setup which does not utilize multipass amplification. The focused intensity from this system is expected to be on the order of $10^{16}$ $\mathrm{W} / \mathrm{cm}^{2}$. Although this system did not exploit the full bandwidth capabilities of type I nearly degenerate OPAs, high gain, conversion efficiency and amplified pulse fidelity in OPCPA were demonstrated. This gives a viable cause for an all OPA-based kilojoule level ultrashort pulse source.

Several improvements should be pointed out that can lead to better performance of an OPCPA system. A nondegenerate OPCPA at $800 \mathrm{~nm}$ would produce better efficiency because of the favorable splitting of the pump energy to the higher frequency field. In 
addition, the availability of ultrashort pulse sources enables the examination of the capability of OPCPA to produce pulses significantly shorter than those possible with this source. The experimental results focused at ultrashort pulse amplification in OPCPA are described in Chapter 5. Finally, the development of appropriate pump lasers with uniform spatial and temporal intensity profile and pulse width matched to the stretched pulse can produce better conversion efficiency. 


\section{Chapter 5}

\section{Nondegenerate optical parametric}

\section{chirped pulse amplification}

This Chapter describes the modeling and experimental effort behind the shortest pulse optical parametric chirped pulse amplifier to date. The highly efficient OPCPA system described in Ch. 4 did not demonstrate the extent of ultrashort pulse capabilities of OPCPA due to the lack of an ultrashort pulse oscillator source and because of the aberrations in the pulse stretcher. Here, recompression of $2 \mathrm{~mJ}$ pulses was achieved to the shortest pulse width of $60 \mathrm{fs}$, at a $10 \mathrm{~Hz}$ repetition rate. The two-stage noncollinear nondegenerate OPCPA pumped by a commercial tabletop pump laser was developed and exhibited broad amplified bandwidth and good stability.

Introductory remarks are followed by the results of numerical modeling of ultrashort pulse capability of OPCPA. A possibility of white light amplification is also addressed in the numerical model. The description of the experimental setup is presented in section 5.4. The experimental data are summarized in section 5.5, which addresses multiple characteristics of the system: extraction efficiency, beam profiles, and recompression. Finally, a perspective is given on the future possibilities of scaling optical pulses to the extremes of peak power. 


\subsection{Motivation}

Generation and amplification of ultrashort laser pulses has been at the forefront of laser research in the recent period. ${ }^{66}$ The development has been driven primarily by newly accessible physics phenomena and applications. CPA allows a wealth of new highfield experiments to performed in the previously inaccessible high-intensity regime. Generation and acceleration of energetic particle beams, ${ }^{67}$ laser-induced nuclear reactions, ${ }^{68}$ and laser-driven cluster fusion ${ }^{69}$ are some of the exciting recent achievements. Short X-ray pulse generation ${ }^{70}$ has been driven by the production of energetic ultrashort pulses from CPA systems. Finally, a great interest exists in the phenomena that occur beyond $10^{21} \mathrm{~W} / \mathrm{cm}^{2}$, the highest intensity achieved to date. ${ }^{9}$

Laser oscillators based on mode-locked Ti:sapphire have produced pulses as short as $5 \mathrm{fs} .{ }^{71}$ On the other hand, CPA in Ti:sapphire has been used to produce pulses shorter than $20 \mathrm{fs} .{ }^{72}$ Maximum peak power from a CPA system of $1.2 \mathrm{PW}$ was achieved using a hybrid Ti:sapphire-Nd:glass laser, ${ }^{8}$ with a pulse duration of $450 \mathrm{fs}$, limited by gain narrowing in Nd:glass. Peak power in CPA systems remains fundamentally limited by the size and damage threshold of the diffraction gratings used in the pulse compressor. Stateof-the-art metallic diffraction gratings ${ }^{43}$ have a maximum diameter of $\sim 1 \mathrm{~m}$, with the damage threshold of $0.5 \mathrm{~J} / \mathrm{cm}^{2}$. This gives a maximum of $\sim 500 \mathrm{~J}$ of energy that can be compressed in a $30-\mathrm{cm}$ beam.

In order to increase the peak power, but surmount the optical damage of diffraction gratings in the pulse compressor, the preferred approach would be to reduce the pulse duration. This leads to a very limited selection of laser materials that allow extremely 
broad bandwidth to be amplified, such as Ti:sapphire ${ }^{50}$ and Cr:LiSAF. ${ }^{73}$ A problem with those materials is their insufficient scalability to large aperture with good optical quality. An additional problem in high peak power systems is the prepulse, ${ }^{74}$ which can be of detrimental effect in applications that include focusing the pulses on targets, thereby producing extreme intensities. OPCPA offers a way to overcome those difficulties through its ultrabroad bandwidth capability (section 3.3), coupled with the extremely large available apertures of nonlinear crystals such as $\mathrm{KDP}^{75}$ This offers a way to scale the peak power of CPA systems beyond the $1 \mathrm{PW}$ level.

OPAs have been shown to be capable of amplifying pulses as short as $5 \mathrm{fs}^{76,77}$ through group velocity matching by tilting the pulse front. ${ }^{78}$ Noncollinear geometry can produce a very broad bandwidth, ${ }^{79}$ which was used in femtosecond, thin-crystal OPAs. However, short-pulse amplification capability of relatively thick $(\sim \mathrm{cm})$ crystals used in OPCPA remains largely unexplored. In a study of the extent of gain bandwidth, ${ }^{61}$ a glimpse of possible ultrashort pulse amplification was offered, but no recompression has been performed. The first degenerate OPCPA experiment ${ }^{12}$ showed pulse recompression to $70 \mathrm{fs}$, but the stretching ratio was only $\sim 18$, allowing a very small energy to be extracted from the pump pulse. Subsequent nearly degenerate experiments with large stretching ratios and large pump lasers produced recompressed pulses of $\sim 280 \mathrm{fs},{ }^{65}$ and 900 fs when pumped by tabletop Q-switched pump lasers. ${ }^{61}$ The experiment described in Chapter 4 produced 310 fs pulses when pumped by a tabletop Q-switched pump laser. A highly efficient OPCPA in periodically poled lithium niobate pumped by a fiber laser ${ }^{80}$ produced $680 \mathrm{fs}$ pulses. In all cases with large stretching ratios, recompression far worse 
than the transform limit was attributed to the uncompensated higher order phase in the stretcher-compressor pair.

Future attractiveness of OPCPA for generation of extreme peak power is then determined by the intrinsic characteristic of OPA to impart a minimal spectral modification and a minimal spectral phase on the chirped pulse, allowing recompression to much shorter pulses than those obtainable from energy-scalable Nd:glass. It is the intention of the experiment performed here to establish this capability of OPCPA. As an additional benefit of the study, the first pulse compression in a highly degenerate OPCPA is demonstrated, which will have an impact on future OPCPA systems that will use highly efficient pump-to-signal energy branching ratios.

\subsection{Numerical modeling of simple ultrashort pulse OPCPA}

Since an ultrashort pulse CPA system requires an ultrashort pulse laser oscillator, the availability of signal wavelengths is limited. The most practical ultrashort pulse solid state source is a Ti:sapphire oscillator, operating near $800 \mathrm{~nm}$. Pumped by a common 532 $\mathrm{nm}$ pump laser, noncollinear OPCPA in BBO exhibits a broad bandwidth around $850 \mathrm{~nm}$, covering the wavelength region near $800 \mathrm{~nm}$. In the experiment presented here, an 820 nm oscillator was used, and the calculations are based on that center wavelength.

In KDP, OPCPA at a practical pump wavelength of $527 \mathrm{~nm}$ (2nd harmonic of Nd:glass) was found to produce a relatively narrow bandwidth $(<1 \mathrm{~nm})$ for all wavelengths far from degeneracy. As an alternative, amplification of pulses centered near $550 \mathrm{~nm}$, pumped by a $351 \mathrm{~nm}$ (3rd harmonic of Nd:glass) shows a broad bandwidth. 
While the generation of $550 \mathrm{~nm}$ seed short pulses may require a nontrivial approach, the possible reward may be the increase in the maximum focusable intensity at a shorter signal wavelength. Another possibility for short-pulse amplification in $\mathrm{KDP}$ is in a degenerate system at $700 \mathrm{~nm}$ signal wavelength, also pumped at $351 \mathrm{~nm}$.

In both nonlinear materials, attractiveness of nondegenerate approach stems from generally broader bandwidth that can be obtained in noncollinear geometry for the same crystal length, and a favorable energy splitting ratio to signal, which improves the conversion efficiency to signal. As a disadvantage of noncollinear geometry, it is necessary to point out a large noncollinear walk-off of the three beams in a relatively long crystal, limiting the approach to higher peak power, high-energy pump pulses.

A short-pulse oscillator is assumed to produce $10 \mathrm{fs}$ transform-limited pulses, with a sech ${ }^{2}$-temporal shape and a spectral bandwidth of $71 \mathrm{~nm}$ FWHM at a center wavelength of $820 \mathrm{~nm}$. We assume a three-stage system based on BBO crystals, pumped at an intensity of $500 \mathrm{MW} / \mathrm{cm}^{2}$ by a $532 \mathrm{~nm}$ pump with a top-hat temporal profile and a pulse width of $3 \mathrm{~ns}$. A $1 \mathrm{~nJ}$ seed pulse is stretched to $1 \mathrm{~ns}$ FWHM, so that the vast majority of the seed pulse energy is contained in the temporal window defined by the pump pulse. The external noncollinear angle between the pump and the signal is $3.73^{\circ}$, maximizing the gain bandwidth. The first two crystals are $12.2 \mathrm{~mm}$ long, producing a total gain of $10^{6}$, with both crystals operating in the small signal regime. Idler is discarded after amplification in each crystal.

Numerical results for spectral amplitude and phase and recompression in the first two crystals is shown in Fig. 5.1. Some spectral narrowing occurs in the amplifiers, with a shift of signal to longer center wavelengths. This is consistent with the calculated 
spectral bandwidth of $\mathrm{BBO}$, which shows the broadest bandwidth near $850 \mathrm{~nm}$ and higher gain at longer wavelengths (Fig. 3.5 (a)). The acquired spectral phase of the amplified pulse in Fig. 5.1 (b) is cumulative by simple addition of phase from individual stages of amplification. Recompressed pulse in Fig. 5.1 (c) is longer after amplification and exhibits prepulses. This is a result of the relatively sharp spectral cut-off and rapidly varying phase at the shorter wavelength region of the amplified signal spectrum. Recompressed pulses are $16 \mathrm{fs}$ and $18 \mathrm{fs}$ long after the first and the second OPA, respectively. Recompressed pulse contrast in Fig. 5.1 (d) is $4 \%$ and $12 \%$ after the first and second OPA, respectively.
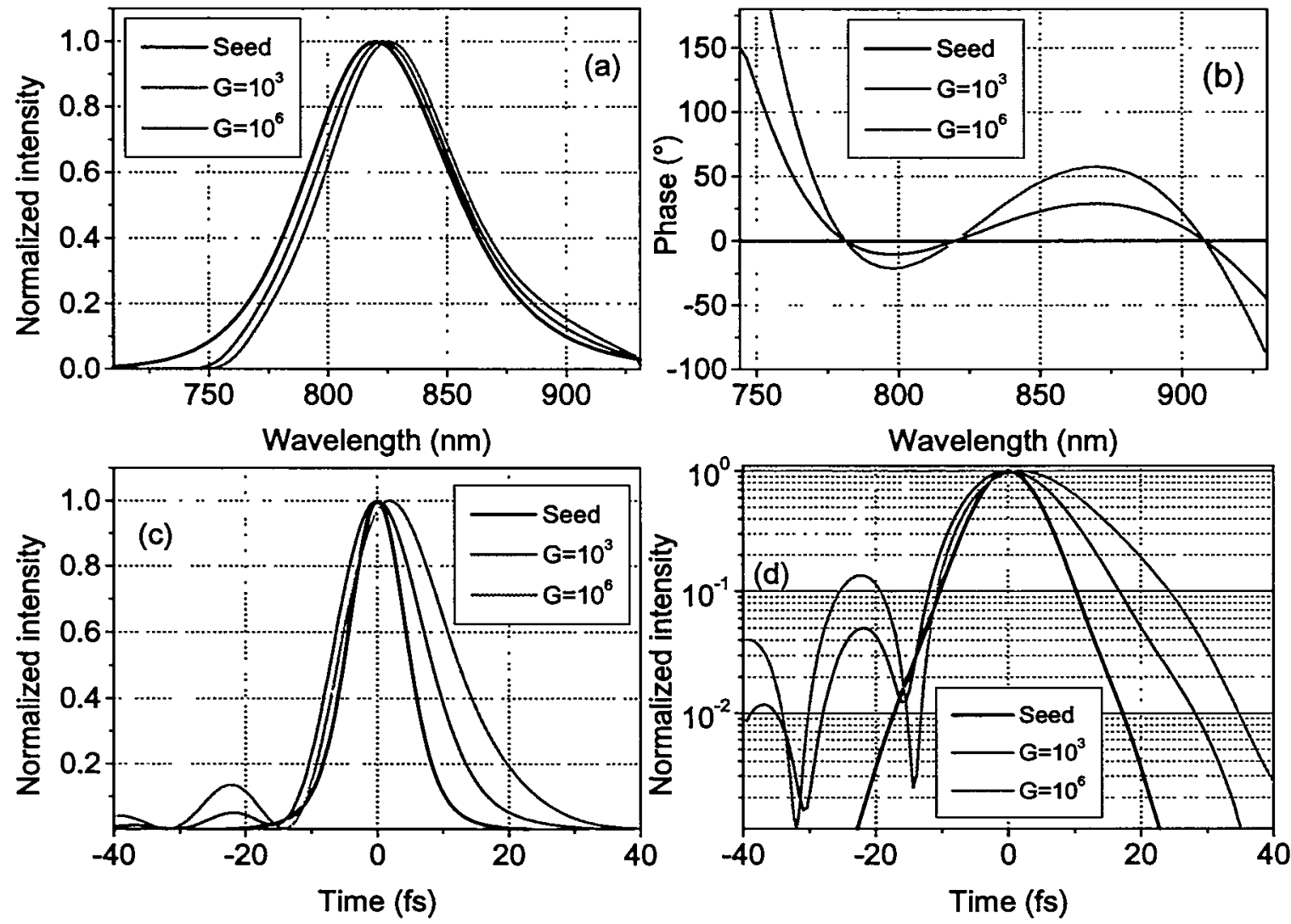

Figure 5.1. Ultrashort pulse amplification in two BBO crystals in the small signal regime. The graphs show (a) spectral intensity, (b) spectral phase, (c) recompressed pulse intensity on linear scale, (d) recompressed pulse intensity on logarithmic scale. Linear dispersion is not included in the calculation. 

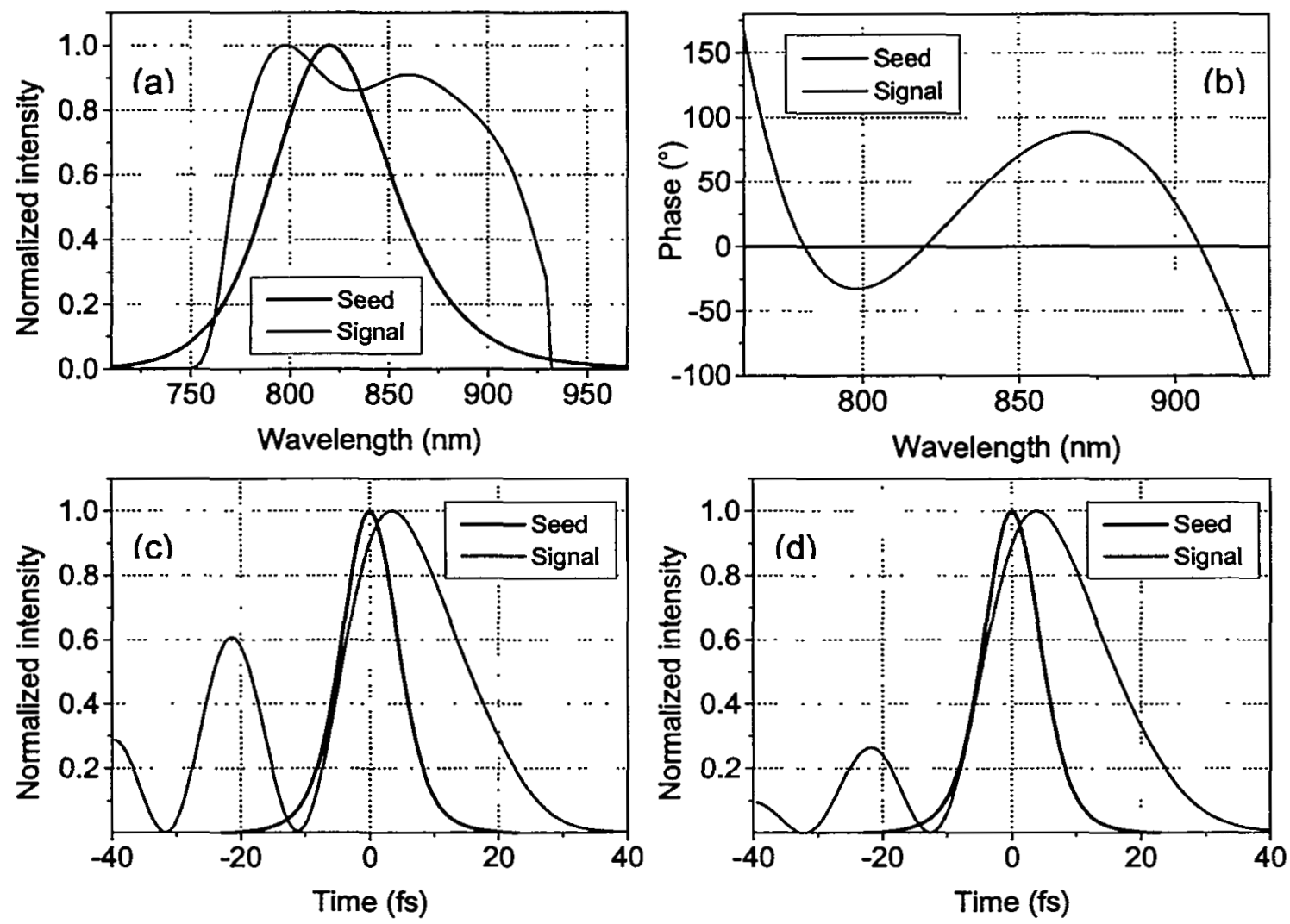

Figure 5.2. Ultrashort pulse amplification in one $B B O$ crystal in the regime of depleted pump. The graphs show (a) spectral intensity, (b) spectral phase, (c) recompressed pulse temporal profile for a $13 \mathrm{~mm}$ crystal, and (d) recompressed pulse temporal profile for a $10 \mathrm{~mm}$ crystal.

The third BBO crystal is pumped by $1 \mathrm{~J}$ of $532 \mathrm{~nm}$ pump, seeded by the output of the second BBO crystal, and it operates in a depleted pump regime. Optimal crystal length for energy extraction is determined to be $13 \mathrm{~mm}$. As the results in Fig. 5.2 (a) indicate, a strong modification of the spectral profile occurs in the depleted amplifier as a result of temporally (spectrally) varying parametric conversion rate, with a relatively large phase as shown in Fig. 5.2 (b). Recompressed pulse in Fig. 5.2 (c) is 19 fs long and has an unacceptable prepulse level of $60 \%$. If the crystal is shortened to $10 \mathrm{~mm}$, the pulse 
contrast is somewhat improved (26\%) as shown in Fig. 5.2 (d), but the extracted energy is reduced to only $40 \%$ of the energy in Fig. 5.2 (c). The reason for improvement of the pulse contrast is the reduced modification of the amplified signal spectrum when the degree of pump depletion is reduced.

Several modifications are suggested to improve the ultrashort pulse amplification performance in a simple BBO OPCPA pumped by a $532 \mathrm{~nm}$ pump. The use of an oscillator centered at $850 \mathrm{~nm}$ takes advantage of a relatively flat and symmetric spectral amplitude and phase in noncollinear BBO OPA, allowing amplification of shorter pulse and an improvement of pulse contrast. The use of a pump beam with tailored back conversion in the amplifier that precedes the last amplifier can improve the amplified spectral shape, extraction efficiency and contrast. Compensation of spectral phase can be performed using standard CPA techniques for linear dispersion, such as prism pairs, mixed gratings, and tailored additional length of material, such as the optical fiber.

\subsection{Angular dispersion and temporal modulation}

\section{for ultrabroad bandwidth in OPCPA}

Since a motivation exists to use OPCPA for amplification of very short pulses, it is important to identify the sources of spectral narrowing and spectral phase shown in the previous section. The primary "intrinsic" source of spectral narrowing and spectral phase is the wave vector mismatch $\boldsymbol{\Delta k}$. It is apparent that the gain bandwidth can be increased simply by using the same length of gain medium, but separated into shorter stages with 
idler separation between the stages. This leads to a complicated setup and does not eliminate the problem of the cumulative spectral phase.

The other characteristic of a zero-lifetime amplification process is the influence of the pump temporal profile on the spectral profile of the amplified pulse. This is especially pronounced in the case of strong pump depletion in high-efficiency extraction. This problem can be suppressed by careful tailoring of the spectral/temporal profile of the seed and/or pump pulse. ${ }^{81,82}$ Previous calculations assumed perfectly collimated seed beams propagating through the crystal at a specific noncollinear angle that maximizes the gain bandwidth. The idea of using noncollinear phase matching for a single wavelength can be extended to the use of angular dispersion of the broadband seed to perfectly phase-match the entire signal spectrum. A similar method has been successfully used previously for broadband frequency doubling. ${ }^{83}$ As an example of the required angular dispersion, Fig. 5.3 shows the calculated noncollinear angle as a function of wavelength for a $532 \mathrm{~nm}$ pumped BBO OPA that amplifies 10 fs FWHM sech ${ }^{2}$-pulses centered at $800 \mathrm{~nm}$, with a spectral bandwidth of $71 \mathrm{~nm}$.

At a slightly greater noncollinear angle for the center wavelength than the one used in broadband amplification, a perfect wave vector matching can be achieved over the entire bandwidth of the signal pulse with a relatively linear angular dispersion. It is anticipated that this dispersion can be readily produced using one or a combination of standard dispersive optical elements, such as prisms. In addition, such angular dispersion could be easily reproduced in a multi-crystal system by relay imaging.

The net result of the use of angular dispersion is the elimination of spectral phase and a very broad bandwidth. The dependence of gain on the signal wavelength can still 
modify the spectral shape of the amplified signal. In Fig. 5.4, a small spectral shift occurs even for a temporally "flat" pump. If it becomes necessary to compensate for this effect, temporal shaping of the pump pulse can be used. This is particularly recommended when OPCPA works in the regime of strong pump depletion. In that case, pump and seed shaping can be used to arrive with the desired spectral shape of the amplified pulse. When longer pulses are used, back conversion in an intermediate stage of amplification can be used to provide the seed with the correct temporal shape for high-energy extraction from the final amplifier.

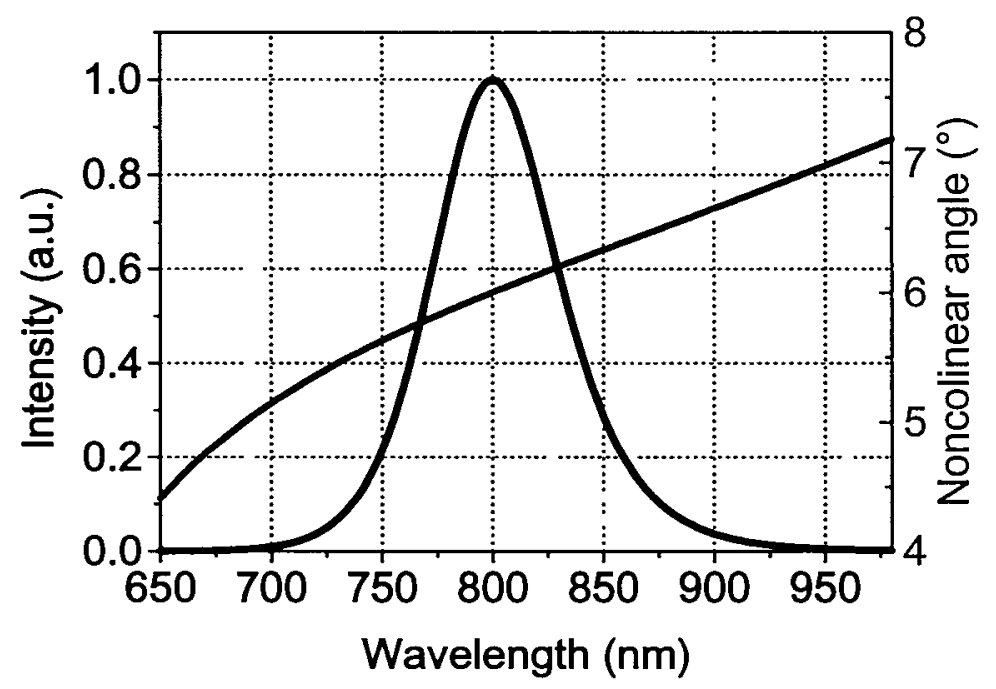

Figure 5.3. Intensity spectrum (black) for a $10 \mathrm{fs}$ pulse centered at $800 \mathrm{~nm}$, and noncollinear angle (red) required to achieve perfect wave vector matching. OPA is pumped at $532 \mathrm{~nm}$. The entire spectral range can be covered by a relatively linear angular dispersion function.

The idea of using angular dispersion for wave vector matching can be taken even further to conceive an OPA-based amplifier capable of amplifying the entire visible spectral range (white light). Such amplifier could be pumped by angular multiplexing of delayed pump beams centered at different wavelengths, allowing the idler to remain 
within the transparency range of the crystal for all wavelengths. The calculations in this and the previous section did not take into account the linear dispersion of the gain medium. Compensation of linear dispersion can be also approached by the use of angular dispersion in OPA.

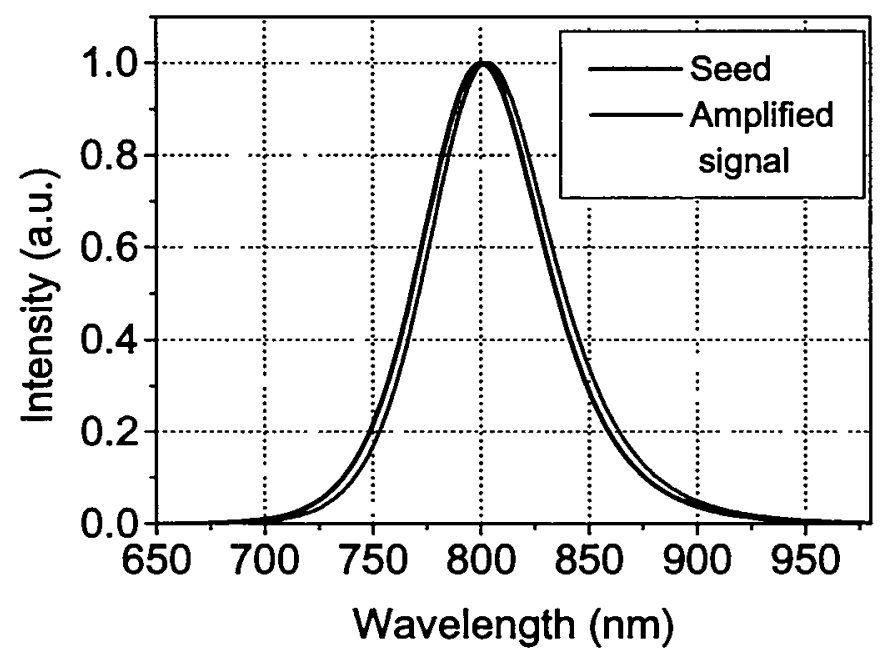

Figure 5.4. A small spectral shift occurs in amplified signal due to the wavelength-dependent gain in OPA. A temporally constant pump is used at $532 \mathrm{~nm}$.

\subsection{Experimental setup}

Fig. 5.5 depicts the schematic of the ultrashort pulse demonstration experiment, which utilizes some of the components of the earlier design of the Falcon laser system. ${ }^{84}$ Seed pulses are obtained from a Ti:sapphire oscillator (Femtolaser Femtosource 20HP), with the center wavelength of $820 \mathrm{~nm}$. The oscillator pulses have a $31 \mathrm{~nm}$ FWHM spectral bandwidth, and their transform-limited FWHM pulse width would be 20 fs. Interferometric autocorrelation of the pulses reveals the pulse width of $33 \mathrm{fs}$ (Fig. 5.6), which is the result of uncompensated dispersion in the output coupler. A broadband pulse 
selector slices single pulses from the oscillator at a $10 \mathrm{~Hz}$ repetition rate.

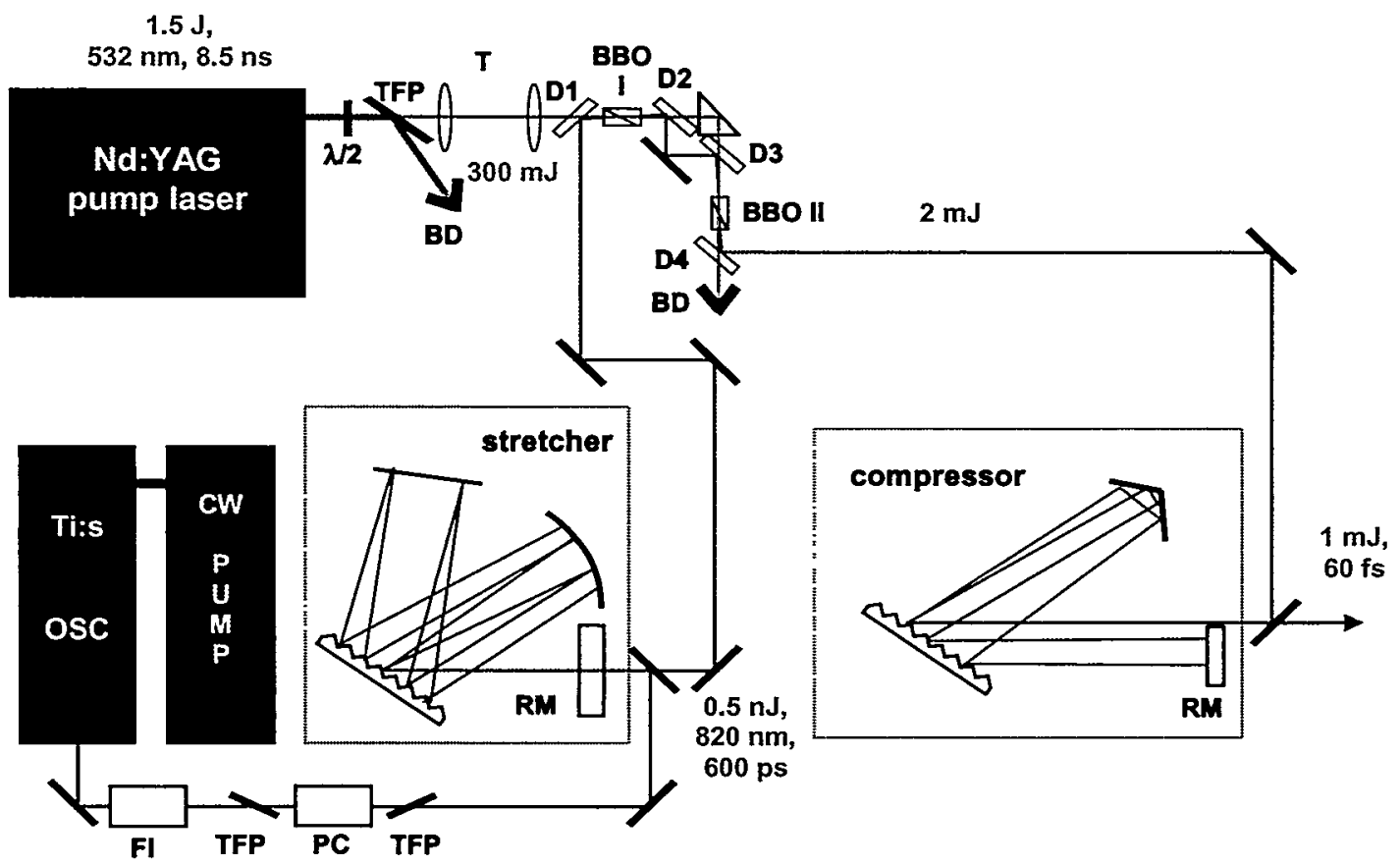

Figure 5.5. Experimental nondegenerate OPCPA setup for ultrashort pulse demonstration. $\lambda / 2$-waveplate, TFP-thin film polarizer, BD-beam dump, FIFaraday isolator, PC-Pockels cell, RM-roof mirror, T-telescope.

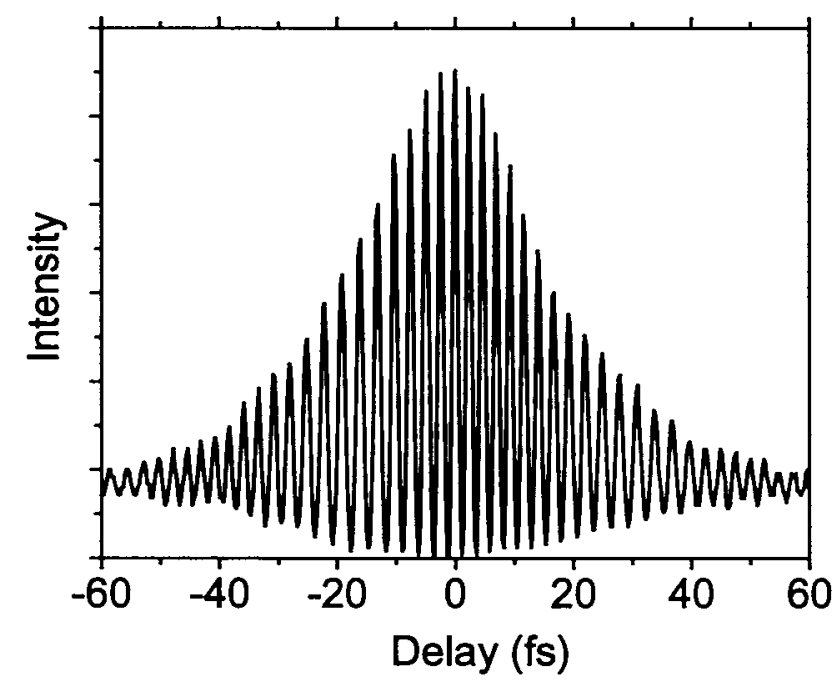

Figure 5.6. Interferometric autocorrelation of the oscillator pulse. FWHM pulse width is $33 \mathrm{fs}$. 
An advanced low-aberration all-reflective design is used for the pulse stretcher. ${ }^{85}$ The stretcher uses a 1480 lines/mm gold diffraction grating. A concave spherical reflector is used in combination with a flat mirror reflector and a mirror-stripe reflector on the diffraction grating to provide positive group delay. Spectral clipping occurs in the stretcher at the bandwidth of $>100 \mathrm{~nm}$. The stretched $1 \mathrm{~nJ}$ pulses are $600 \mathrm{ps}$ long at their FWHM.

The OPA in this experiment consists of two BBO crystals. The dimensions of the two crystals are $7 \times 7 \times 15 \mathrm{~mm}^{3}$ and $5 \times 5 \times 15 \mathrm{~mm}^{3}$. The crystals are cut at $23.8^{\circ}$ for type I phase matching, and they have $2^{\circ}$ wedges on their output faces for suppression of parasitic oscillation. They are antireflection-coated for $532 \mathrm{~nm}, 820 \mathrm{~nm}$, and $1.6 \mu \mathrm{m}$.

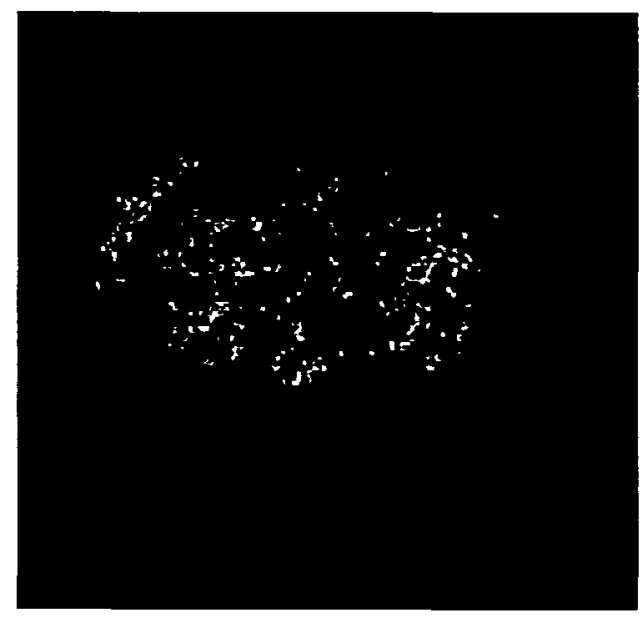

Figure 5.7. Near-field transverse intensity profile of the pump pulse (Spectra-Physics GCR Pro 350-10)

A commercially available Q-switched Nd:YAG laser is used for pumping the OPA. The laser is a Spectra-Physics GCR Pro 350-10, upgraded with an injection seeder for single longitudinal mode operation. The laser produces $1.5 \mathrm{~J}$ of $532 \mathrm{~nm}$ pulses, with a 
pulse width of $6.9 \mathrm{~ns}$, at a $10 \mathrm{~Hz}$ repetition rate. The supergaussian near-field transverse intensity profile of the pump beam (Fig. 5.7) is advantageous for pumping the OPA. This laser also exhibits spatio-temporal evolution characteristic for an unstable resonator. ${ }^{59}$ The procedure described in section 4.2 was used again to evaluate the characteristics of the pump pulse. The numerical result of the fit is shown in Fig. 5.8. This laser exhibits the same qualitative behavior as the previously used Spectra-Physics GCR 270-10 pump laser. Since the stretched seed pulse width in this experiment is 5 times shorter than in the previous experiment, the effect of the pump pulse shape is less important. The pump pulse is slightly elliptical, as a consequence of inefficient frequency doubling in the vertical direction in the pump laser, coinciding with the sensitive angular direction in the frequency doubling nonlinear crystal.

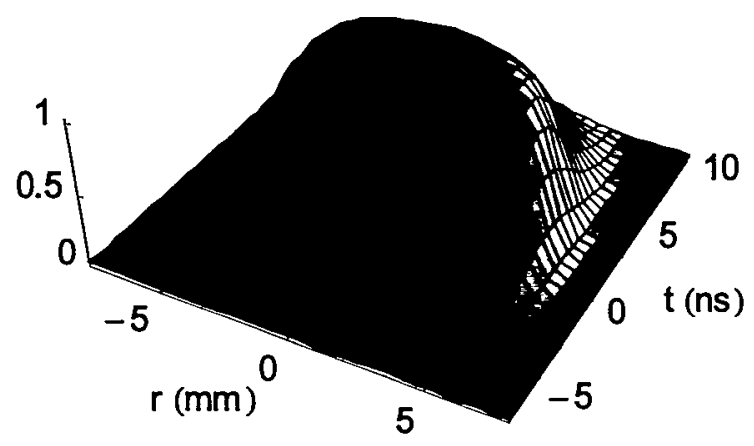

Figure 5.8. Spatio-temporal evolution of the pump pulse emanating from an unstable resonator in Spectra-Physics GCR Pro 350-10 laser

The $3.2^{\circ}$ walk-off of the pump beam is not the dominant factor that reduces the beam overlap in noncollinear OPA. To allow broad bandwidth amplification in BBO, a $3.7^{\circ}$ external noncollinear angle is used between the signal and the pump beam. This 
implies a strong noncollinear walk-off effect over the $15 \mathrm{~mm}$ crystal length. In addition, the idler beam walk-off occurs with an external angle of $7.4^{\circ}$. The noncollinearity of the signal and pump is achieved in the plane perpendicular to the crystal principal plane. In this way, the cumulative effect of two different sources of walk-off is reduced.

$300 \mathrm{~mJ}$ is split from the pump pulse to pump the OPA. Pump energy can be continuously adjusted using a waveplate-polarizer combination. A vacuum telescope relays the supergaussian transverse intensity profile between the two OPA crystals in a collimated beam. The diameter of the pump beam in the OPA is $3 \mathrm{~mm}$, with a peak intensity of $500 \mathrm{MW} / \mathrm{cm}^{2}$. The pump pulse after the first crystal is essentially undepleted, except for the energy lost on dichroic coating inefficiencies. The same pulse is used to pump the second crystal, with a peak intensity of $470 \mathrm{MW} / \mathrm{cm}^{2}$. The seed beam diameter is $1 \mathrm{~mm}$ at its FWHM, and it has a gaussian spatial profile. Signal and idler are spatially separated after each amplification stage. Fig. 5.9 shows photograph of the nondegenerate OPCPA experimental setup.

The pulse compressor contains a 1480 lines/mm grating, set at a diffracted angle near $55^{\circ}$. Fine tuning of the compressor grating angle is used to reduce the amount of residual phase in the system. The most significant source of uncompensated quartic and quintic phase is the stretcher with its spherical mirror. No compensation is necessary for B-integral, since the path length through the gain medium is very short. The compressor passes the entire amplified spectrum, with an efficiency of $50 \%$. A simple single-shot second-order intensity autocorrelator measures the pulse width of the recompressed pulse. 


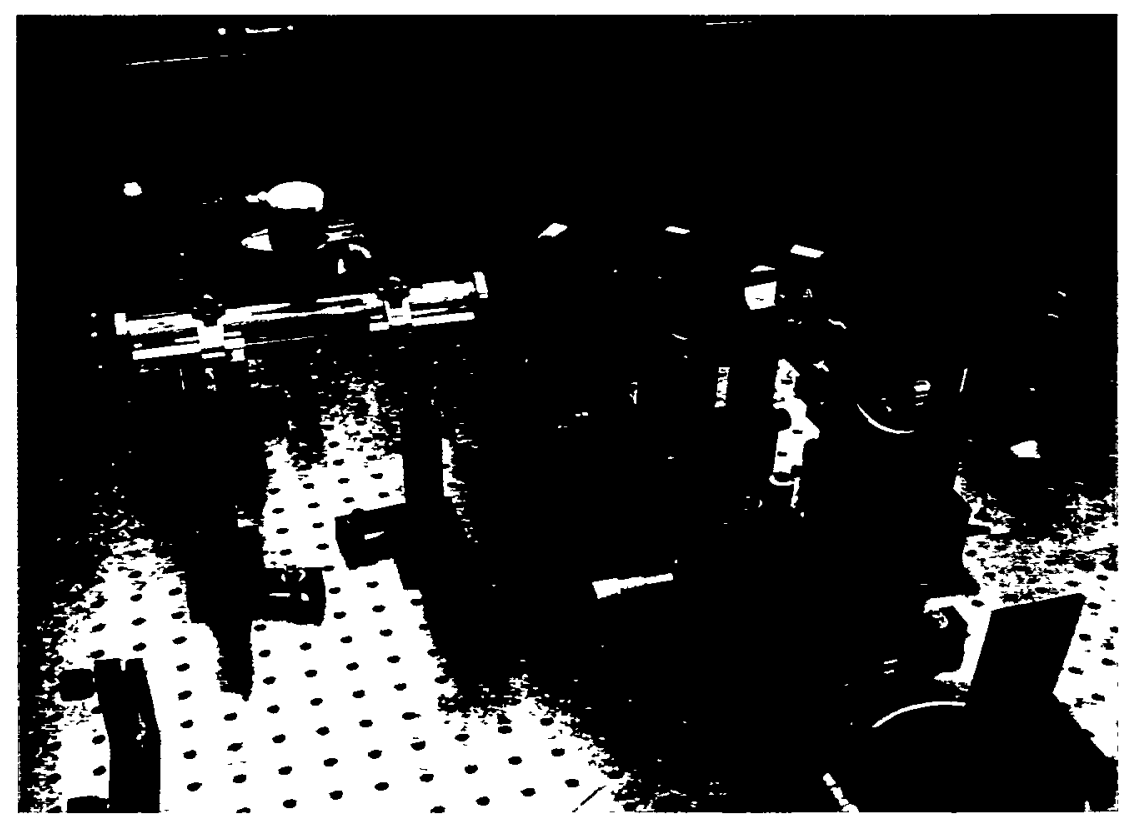

Figure 5.9. Nondegenerate OPCPA setup

\subsection{Experimental results}

The maximum gain obtained from the two-crystal system was $4 \times 10^{6}$, resulting in 2 $\mathrm{mJ}$ pulses amplified in a single pass through only $30 \mathrm{~mm}$ of gain material. A poor overall pump to signal conversion of $0.7 \%$ was obtained in this configuration, resulting from the small temporal overlap of the signal and the pump pulse.

Spectral bandwidth was measured for stretched seed, and amplified signal at two different pump energies, resulting in $0.5 \mathrm{~mJ}$ and $2 \mathrm{~mJ}$ pulses from the OPA. The resulting spectra are shown in Fig. 5.10. A shift of the center wavelength to $830 \mathrm{~nm}$ is observed, which is consistent with the gain bandwidth in nondegenerate BBO OPA, which is centered at longer wavelengths (near $850 \mathrm{~nm}$ ), and with the increase of gain with wavelength near $820 \mathrm{~nm}$. The small bandwidth narrowing $(<2 \mathrm{~nm})$ at the FWHM 
observed when the OPA operates far below saturation $(0.5 \mathrm{~mJ})$ can be attributed to this spectral shift. At the point near saturation $(2 \mathrm{~mJ})$, the spectrum is modified further and the amplified FWHM is increased to $35 \mathrm{~nm}$. Spectral broadening is the result of different rate of nonlinear conversion for the spectral components of different initial intensity.

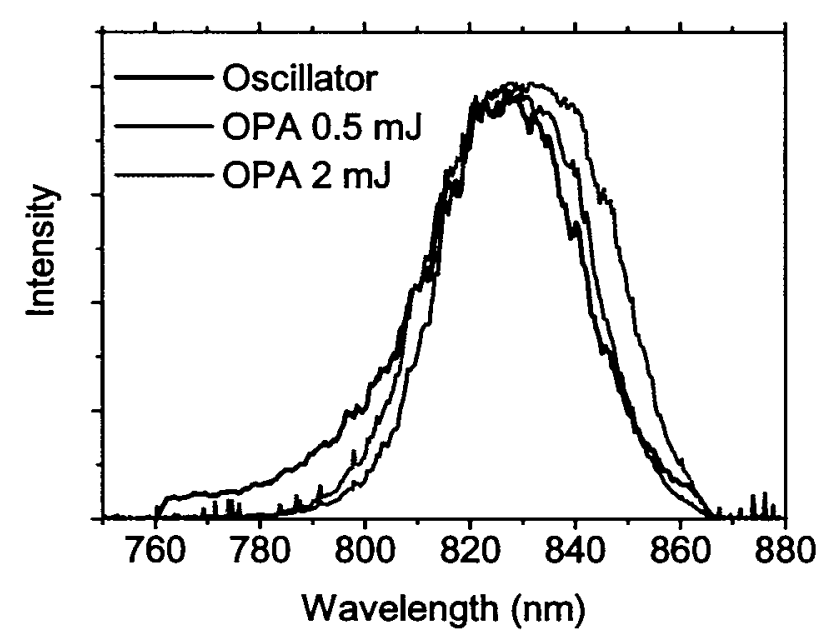

Figure 5.10. Spectral profiles of the seed and amplified signal at two energy levels. Notable is the increase of FWHM spectral bandwidth when the OPA operates near saturation (green).

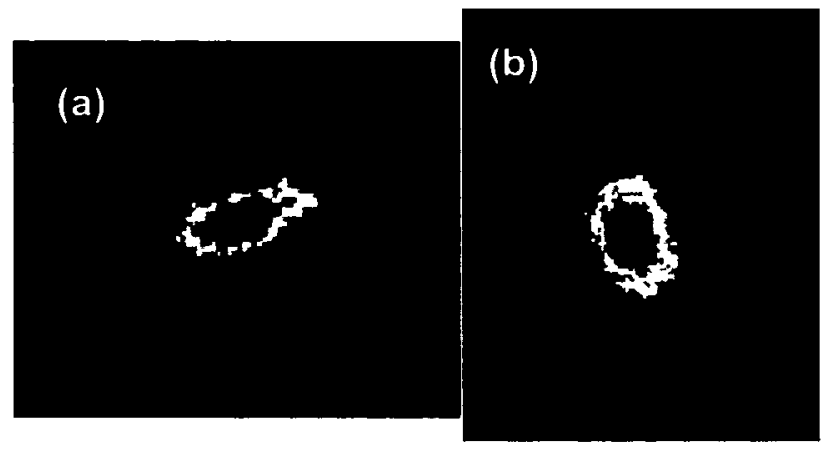

Figure 5.11. (a) Near-field and (b) far-field transverse intensity profiles for the signal at the maximum energy from the nondegenerate OPCPA 
Near-field and far-field transverse intensity were recorded for amplified signal, and they are shown in Fig. 5.11. Some ellipticity is observed in the beam profiles, which is possibly originating from the elliptical seed beam in the near field, combined with the noncollinear walk-off of signal and idler in OPA. The ellipticity in the seed beam is the result of astigmatism in the oscillator and stretcher.

Pulses were recompressed and the result of the intensity autocorrelation at the full output energy is presented in Fig. 5.12. A careful optimization of the stretcher and compressor pair produced the autocorrelation trace in Fig. 5.12. The measured FWHM autocorrelation of the recompressed pulse is $104 \mathrm{fs}$, which is nearly 2 times longer than the FWHM of the calculated autocorrelation of the transform-limited pulse with the measured spectrum (Fig. 10). With the inclusion of the spectral phase in the system, ${ }^{86}$ the calculated FWHM of the autocorrelation is $108 \mathrm{fs}$, which is within our experimental error, indicating that we produced 60 -fs pulses. These pulses are the shortest pulses ever produced in OPCPA. The calculated shape of the recompressed pulse using the spectrum and calculated phase is shown in Fig. 5.13 (a), indicating 52.4 fs pulses after recompression. In Fig. 5.13 (b), the calculated recompressed pulse shape is shown with the spectral phase removed. In that case, bandwidth-limited recompressed pulse width in this system would be 29.4 fs. The recompressibility limit in this experiment is the uncompensated quartic and quintic phase originating primarily in the stretcher, which employs a spherical lens designed to cancel the high-order dispersion in a system which includes more gain material. A calculation shows that $425 \mathrm{~cm}$ of fused silica would compensate the majority of high-order dispersion and allow compression to $32.7 \mathrm{fs}$. 


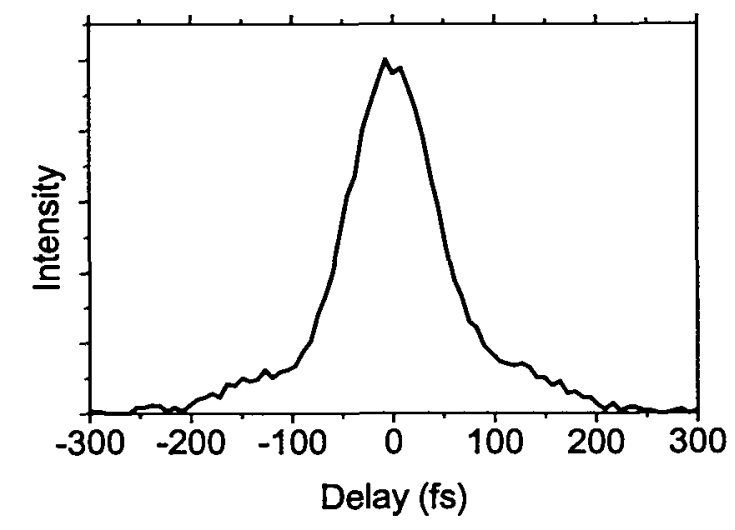

Figure 5.12. Recompressed pulse intensity autocorrelation. Deconvolved pulse width is $60 \mathrm{fs}$.
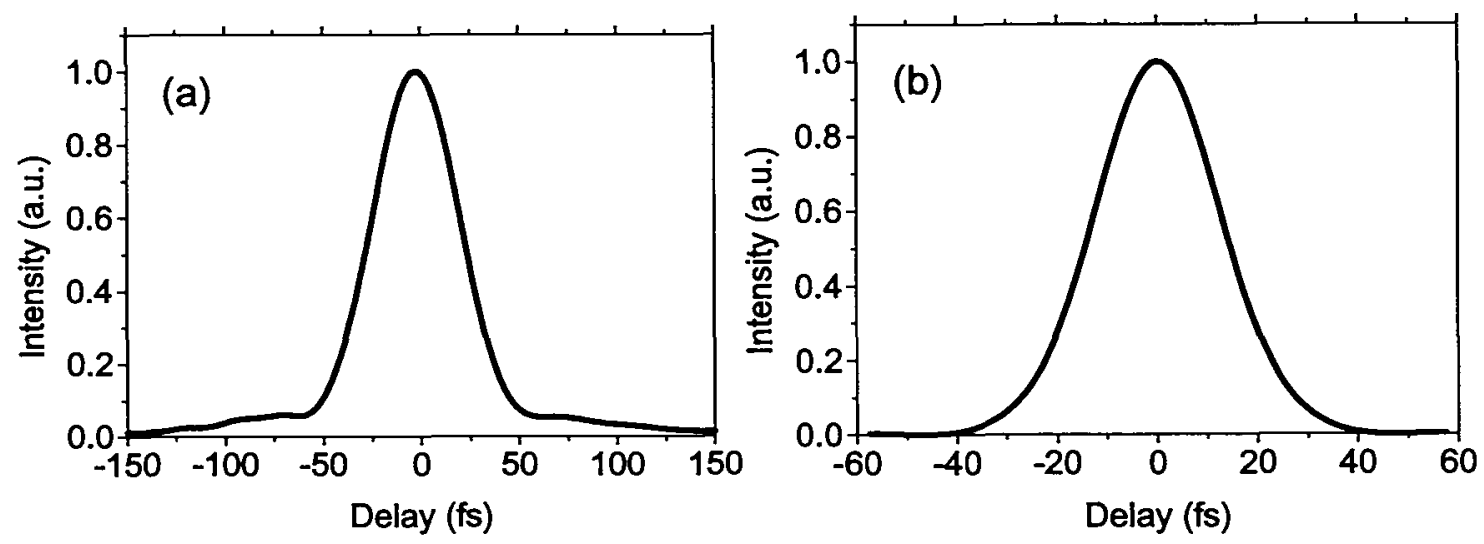

Figure 5.13. Calculated recompressed pulse shape with (a) total calculated spectral phase in the system, and (b) all spectral phase removed. FWHM pulse width is (a) $52.4 \mathrm{fs}$, and (b) $29.4 \mathrm{fs}$.

\subsection{Conclusion}

In this experiment, the capability of OPCPA to amplify ultrashort pulses was demonstrated at a level not achieved in any previous experiment. The $60 \mathrm{fs}$ recompressed pulses are nearly five times shorter than the pulses obtained in previous experiments that used appreciable stretching ratios. ${ }^{61,62,65}$ In addition, those short pulses were obtained 
from an OPCPA system pumped by a tabletop commercially available pump laser. This is also the first reported pulse recompression in highly nondegenerate OPCPA.

The pulse energy obtained from this source is smaller compared to the pulse energy obtained in the experiment in Ch. 4. This is the result of the poor overlap between the stretched signal pulse and the pump pulse. However, the conversion efficiency in future optimized nondegenerate systems should be greater than in nondegenerate because of the asymmetric splitting of the pump energy to signal and idler.

This demonstration of the ultrashort capability of OPCPA is a step further towards sources that will produce much higher energy and shorter pulse width compared to existing large glass lasers. Scalability of KDP to large apertures allows scaling of ultrashort pulses (tens of fs) to kilojoule energies, and its use in OPCPA should be evaluated in more detail in the future. In addition, the aperture scaling of other nonlinear crystals suitable for OPCPA can be also pursued in order to increase the possible pulse energy.

Numerical modeling presented in this Chapter points to a way to increase and flatten the spectral bandwidth by use of angular dispersion of the seed beam and pump beam temporal modulation. Pulses shorter than $10 \mathrm{fs}$ can be amplified using this method, which is of great interest for scaling the CPA to pulses shorter than the ones possible by amplification in Ti:sapphire. 


\section{Chapter 6}

\section{Hybrid chirped pulse amplification}

\section{for multi-TW tabletop sources}

OPCPA can exhibit high conversion efficiency only when the seed pulses and the pump pulses are suitably shaped and well matched in space and time. This Chapter introduces a technique that can improve the conversion efficiency in an OPCPA system by using a laser amplifier for efficient conversion of the pump energy which does not get converted in OPCPA. This hybrid system leads to pump-to-signal conversion efficiency of up to $50 \%$, and to significantly relaxed requirements for the pump laser.

An experimental demonstration of the concept was undertaken, with the final result of $45 \mathrm{~mJ}$ of amplified pulse energy in a system pumped by $300 \mathrm{~mJ}$ pulses from a tabletop, frequency doubled Q-switched Nd:YAG pump laser, with $>20 \%$ conversion efficiency of the absorbed pump energy in the Ti:sapphire crystal in three passes. Seed pulses centered at $820 \mathrm{~nm}$ originating in a short-pulse Ti:sapphire oscillator are amplified using OPCPA and a final multipass Ti:sapphire amplifier. Optimized recompression of amplified pulses with the demonstrated bandwidth would produce a peak power of $2 \mathrm{TW}$, at a $10 \mathrm{~Hz}$ repetition rate. This unprecedented combination of gain and conversion efficiency was accomplished without the use of electro-optic modulators, in a relatively simple setup, 
which has a total gain path of $10.5 \mathrm{~cm}$ in a combination of $\beta$-barium borate and Ti:sapphire.

The limitations of tabletop laser-pumped OPCPA are discussed first, and the concept of hybrid chirped pulse amplification is introduced. The experimental setup and results are described in sections 6.3 and 6.4, respectively. Recapitulation of the results and future plans are outlined in the conclusion.

\subsection{Efficiency of tabletop OPCPA}

A common problem that occurs in design of OPCPA systems is the stringent set of requirements imposed on the nanosecond pump laser. The ideal pulse width of the pump pulse is given by the pulse width of the stretched seed pulse, which rarely exceeds $1 \mathrm{~ns}$. The desired spatial and temporal profile of the pump laser is a top-hat, which minimizes spatial and temporal (spectral) modification of the signal. To maximize conversion efficiency, it is desirable that the signal is also shaped as top-hat in space in time. Shaping of the chirped signal as top-hat in time is problematic considering the subsequent $\operatorname{sinc}^{2}$ recompressed temporal shape of a pulse with top-hat spectrum.

Another strategy that can allow large conversion efficiency in OPA was suggested by Begishev. ${ }^{81,82}$ and utilizes gaussian-like shaping of seed and/or pump pulses to achieve high conversion efficiency. Different rates of conversion for different signal and pump intensities can be balanced by achieving back conversion in a preamplifier stage, followed by amplification in the final amplifier stage using a gaussian-like pump. This approach has the advantage that no special pulse shaping techniques are necessary. 
However, the gain material is longer for the same gain, and a careful independent optimization of the preamplifier and the power amplifier is necessary to achieve maximum conversion efficiency.

In broadband optical parametric amplification that occurs in critically phasematched birefringent crystals, the walk-off of the pump beam from the signal and idler beams inside the crystal limits the effective extraction area of the pump beam. In noncollinear OPA, noncollinear walk-off reduces the effective beam overlap even further, particularly because the effect of the noncollinear walk-off is additive to the effect of the birefringent walk-off. As a consequence, a greater beam diameter and higher pump peak powers are required to achieve high conversion efficiency. The effect of birefringent walk-off can be eliminated in noncritical phase matching and quasi phase matching. Splitting the gain length into several short crystals can reduce the effect of both types of walk-off, at an expense of increasing system complexity.

The required beam quality of the pump laser is often close to diffraction limited, determined by the angular acceptance of the OPA. A calculation is performed in Ch. 8 to determine the impact of divergence and beam quality on conversion efficiency in OPA. Divergence can be reduced by using larger beam sizes, which mandates higher pump laser peak power. Beam quality requirements can be also relaxed by splitting the gain length into several shorter crystals.

A motivation exists for using the existing commercial tabletop pump lasers to pump OPCPA systems. When pumping OPCPA, another problematic feature of those lasers is their spatio-temporal evolution and relatively long pulse width. While temporal multiplexing can improve the overall energy extraction, the temporal shape of the pump 
pulses is not a top-hat unless additional pulse shaping is performed. Pulses obtained from unstable resonators $^{59}$ are particularly problematic when used in OPCPA, as they exhibit complicated spatio-temporal evolution associated with radially variable build-up time in the laser resonator and subsequent nonuniform depletion of the laser gain medium.

Experimentally achieved efficiency in OPCPA systems has exceeded $20 \%$ in the spatio-temporal window defined by the seed pulse (Ch. 5, Ref. 65). An overall efficiency of $20 \%$ was achieved in a fiber laser-pumped OPCPA system, ${ }^{80}$ in which a good temporal overlap was achieved between the pump beam and the signal beam. In $\mathrm{Ch}$. 5 , the experiment showed the overall conversion efficiency of $6 \%$ in a system pumped by a tabletop commercial pump laser. These overall efficiencies of OPCPA are typically lower than the highest efficiency that can be obtained from a laser amplifier.

\subsection{The hybrid optical parametric-laser CPA system}

Given the relatively modest conversion efficiency in OPCPA pumped by a tabletop commercial laser, alternative methods of achieving high conversion efficiency can be explored. Here, a hybrid optical parametric-laser CPA system is presented, which can relax the requirements on the pump laser and still achieve high conversion efficiency from a tabletop, long-pulse, Q-switched laser.

The basic principle of a hybrid optical parametric-laser CPA system is shown if Fig. 6.1. The oscillator generates short pulses, which are stretched in time using a pulse stretcher. The pulse width of the pump laser can be significantly longer than the stretched seed pulse width. A high-gain OPA pumped by a tabletop Q-switched laser amplifies the 
stretched seed pulse to the level determined by the temporal and spatial overlap between the stretched signal and the pump pulse. A unique feature of OPA that distinguishes it from laser gain media is the fact that no pump absorption and energy storage occurs in OPA. As a result, the pump energy that remains unconverted in OPA is available for pumping an additional stage of laser amplification. The laser amplifier seeded by pulses amplified in OPA is used to obtain large energy extraction from the residual pump pulse. Recompression of the stretched amplified pulse occurs in pulse compressor.

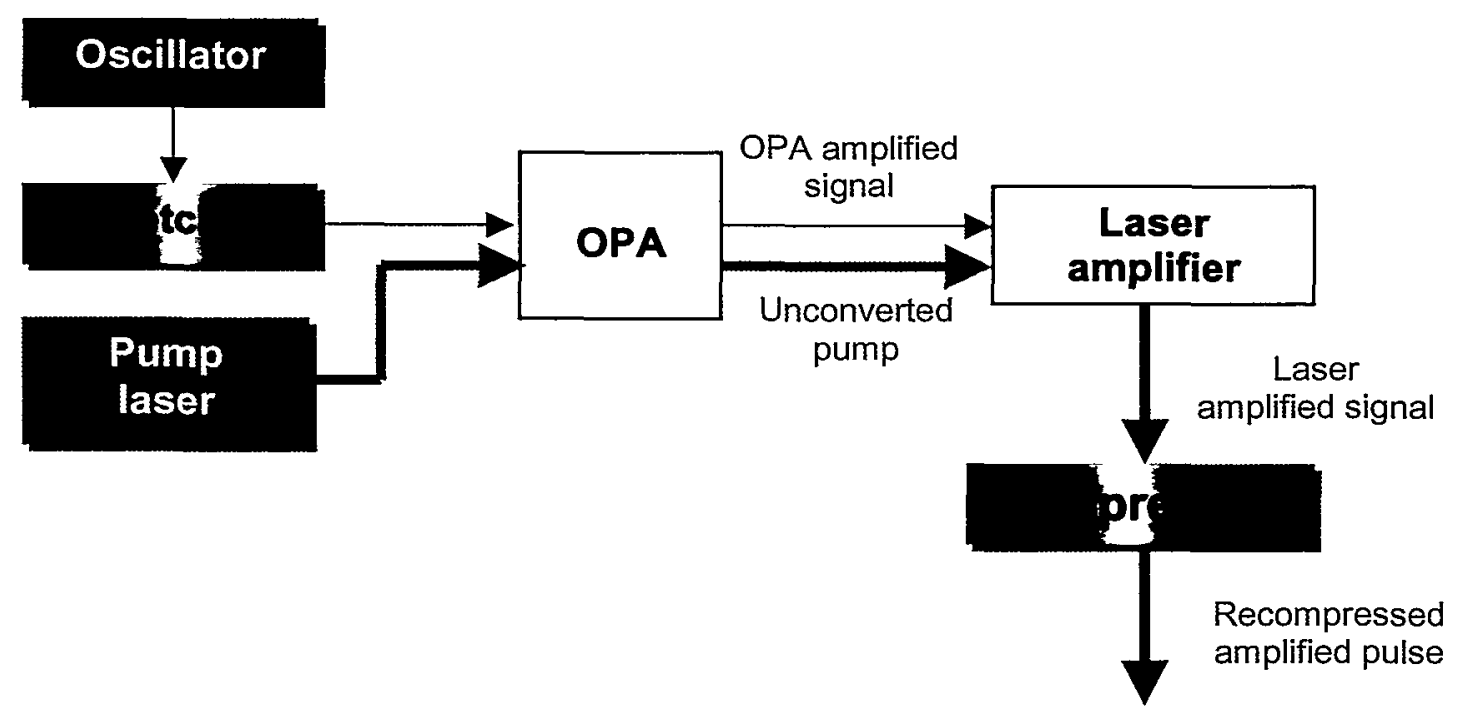

Figure 6.1. The concept of a hybrid chirped pulse amplification

If a small temporal slice of the pump beam was converted in OPA, most of the pump pulse energy is available for pumping the laser amplifier. Laser amplification is insensitive to temporal modulation of the pump pulse after its use in OPA, because laser media store the total integrated pump energy. The gain obtained from OPA is typically comparable to the gain from a standard regenerative amplifier pumped by the same laser.

Pulse amplification in a laser can result in excellent conversion efficiency. As an 
example, conversion efficiency of $50 \%$, or $90 \%$ of the theoretical quantum efficiency, has been reported in Ti:sapphire. ${ }^{87}$ It is expected that the overall conversion efficiency in an optimized hybrid CPA system which utilizes Ti:sapphire can be close to $50 \%$ before compression, which compares favorably with the efficiency that can be achieved in a high-gain regenerative amplifier alone.

In addition to conversion efficiency, hybrid system has other advantages. The length of gain material is reduced. This results in reduced dispersion, reduced B-integral in the system and easier recompression. Electro-optic modulators are not used in a hybrid system which does not employ regenerative amplification, thus significantly reducing system component cost and complexity and improving safety. Finally, prepulses originating from the regenerative amplifier are eliminated, and the hybrid OPA-laser CPA system contrast in the pulse pedestal is limited by a combination of parametric fluorescence and amplified spontaneous emission.

\subsection{System design}

Fig. 6.2 depicts the schematic of the highly efficient hybrid CPA experiment. This experiment is built as an extension of the nondegenerate OPCPA experiment performed in Ch. 5, and its additional laser amplifier is described here. After amplification in the OPA, the maximum obtained signal pulse energy was $2 \mathrm{~mJ}$, while the available residual pump energy from the OPA is $260 \mathrm{~mJ}$ after losses incurred on antireflection coating inefficiencies in the system. This beam is used to pump the final three-pass Ti:sapphire amplifier.

The laser amplifier consists of a single, $25 \mathrm{~mm}$ long Ti:sapphire crystal, which is 
antireflection-coated for the broadband signal wavelength centered at $820 \mathrm{~nm}$. The pump beam is recovered from the second OPA amplifier and relay imaged into the Ti:sapphire crystal, achieving the peak fluence of $3 \mathrm{~J} / \mathrm{cm}^{2}$. Ti:sapphire crystal is pumped from one side. The $2 \mathrm{~mJ}$ seed beam from the OPA is suitably delayed in time through a doublebounce off a set of broadband dielectric mirrors, producing a $\sim 3$ ns delay between the pump and the first pass of signal in Ti:sapphire. In this way, the absorption of the energy in the back (later part) of the pump pulse is achieved before the seed pulse enters the Ti:sapphire amplifier. Simultaneously, oscillator pulses arriving in the multipass amplifier prior to the main pulse are not amplified, since their arrival times are prior to the arrival of the pump pulse. Short fluorescence lifetime of Ti:sapphire $(\sim 3 \mu \mathrm{s})$ precludes amplification of pulses using the energy of the prior pump pulse at the repetition rate of $10 \mathrm{~Hz}$

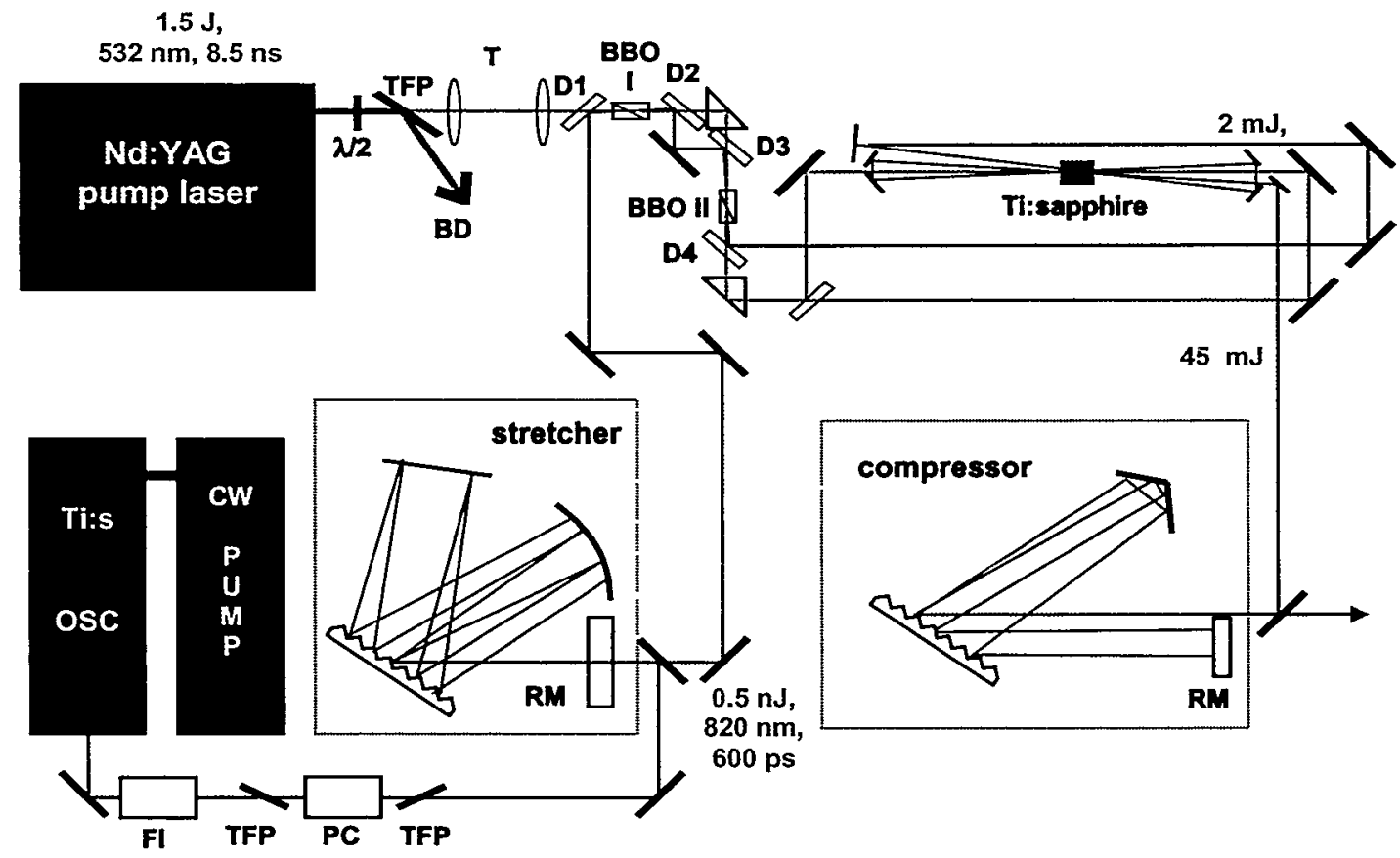

Figure 6.2. Experimental setup for demonstration of hybrid chirped pulse amplification. $\lambda / 2$-waveplate, TFP-thin film polarizer, BD-beam dump, FIFaraday isolator, PC-Pockels cell, RM-roof mirror, T-telescope. 
The seed beam is sent directly into the laser amplifier after collimation using a single lens. This is possible because of the slight divergence of the signal after amplification in OPA. The seed beam slightly overfills the pump beam in Ti:sapphire, with a goal of producing good beam quality. A common bow-tied multipass amplification scheme is used. The angle between the pump and the signal in every pass in the multipass amplifier is minimized to improve the spatial overlap of pump and signal.

\subsection{Experimental results}

The absorption of $532 \mathrm{~nm}$ pump in Ti:sapphire was measured first. From the incident $260 \mathrm{~mJ}$ of pump approximately $200 \mathrm{~mJ}$ were absorbed after taking into account the losses on the crystal surface. The obtained maximum gain in the four passes in the Ti:sapphire amplifier was $2.5,4$, and 2.25. The corresponding pulse energies after each pass were $5 \mathrm{~mJ}, 20 \mathrm{~mJ}$, and $45 \mathrm{~mJ}$. The gain in the first pass is reduced because of the spatial overfill of the pump beam. Far-field beam profiles after the third pass is shown in Fig. 6.3. A relatively good beam quality is the result of amplification, since the Ti:sapphire amplifier acts as a spatial filter on the seed that overfills the pump.

Amplified spectrum was measured, and their comparison with the seed spectrum and the spectrum amplified in OPA is shown in Fig. 6.4. It can be noted that Ti:sapphire slightly shifts the peak of the spectrum back to shorter wavelengths, since its gain peak is near $800 \mathrm{~nm}$. The amplified FWHM bandwidth is $34 \mathrm{~nm}$, slightly narrower than after amplification in OPA. 


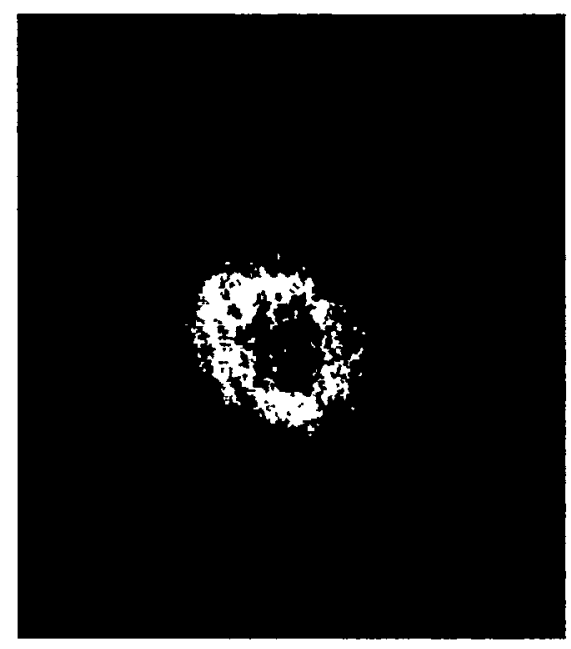

Figure 6.3. Beam profile of the amplified pulse after the third pass in Ti:sapphire amplifier

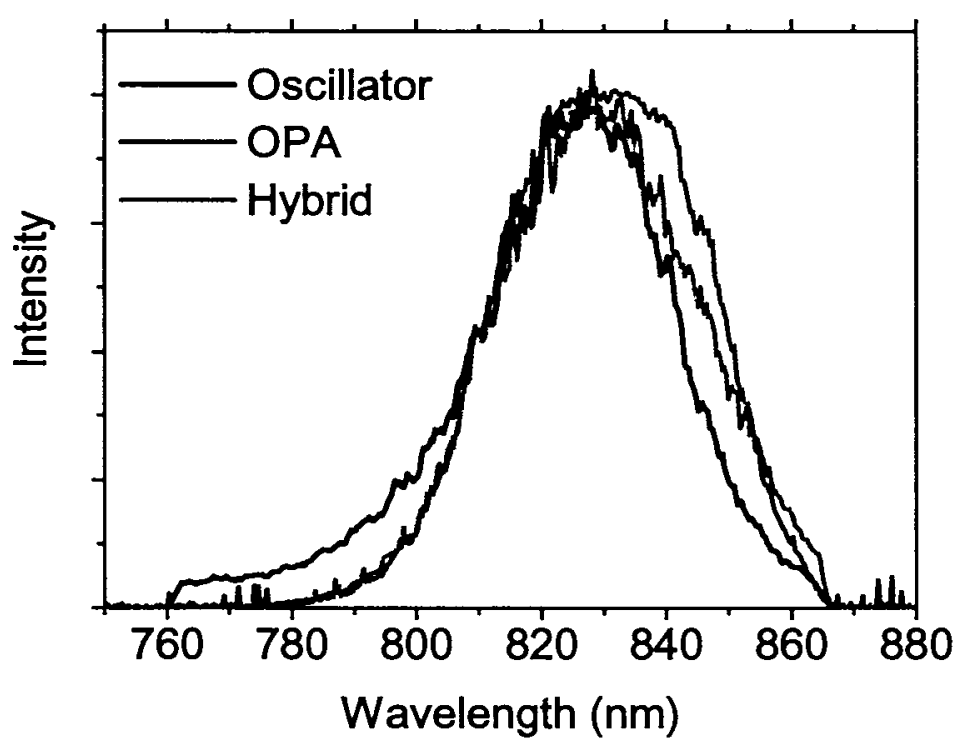

Figure 6.4. Oscillator and amplified signal spectra from the OPA and from the Ti:sapphire multipass amplifier

Hybrid CPA system exhibited high stability, as a result of high saturation both in OPA and in Ti:sapphire amplifier. The photograph of the experimental setup for hybrid CPA is shown in Fig. 6.5. 


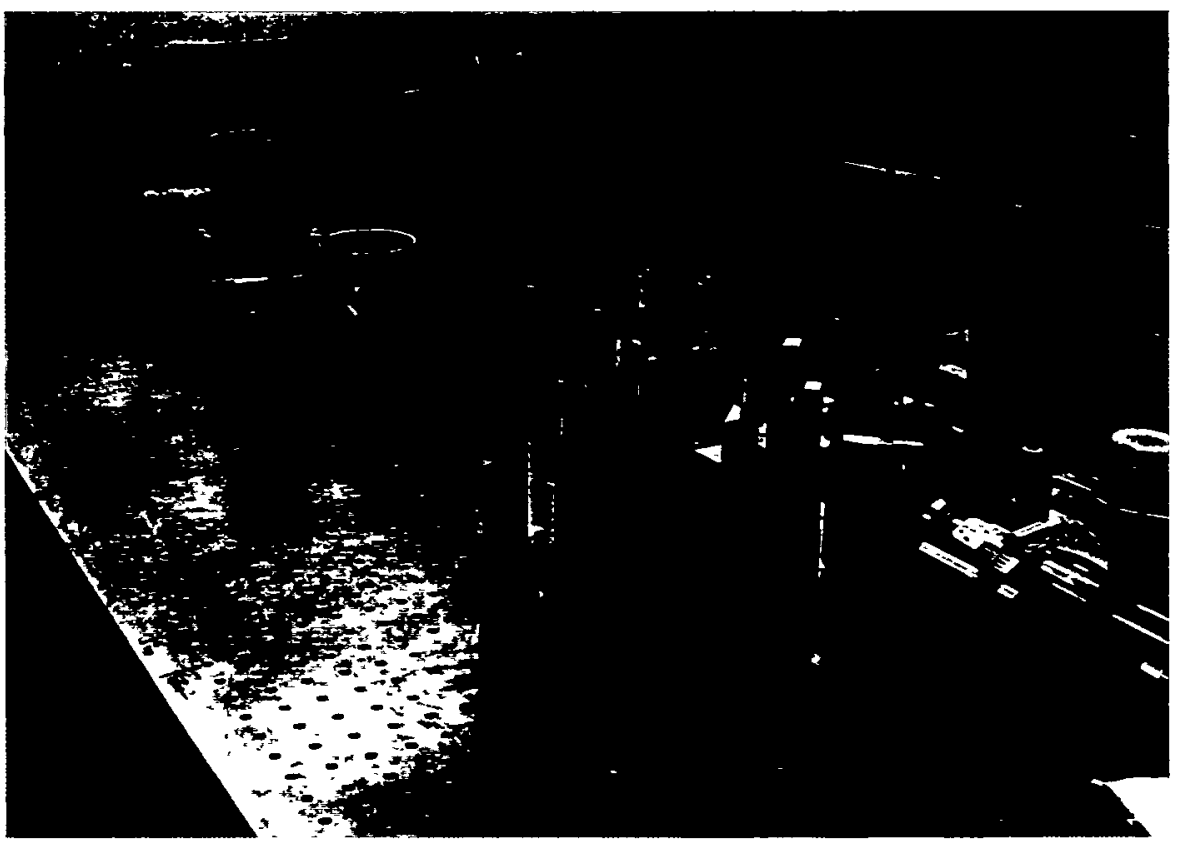

Figure 6.5. The experimental setup of the hybrid CPA experiment.

\subsection{Conclusion}

This experiment demonstrates the successful use of a novel hybrid CPA architecture based on $\mathrm{BBO}$ and Ti:sapphire which does not require electro-optic modulators and exhibits superior energetic performance when compared to a system based on OPA alone and superior bandwidth and simplicity when compared to a system based on Ti:sapphire alone. The demonstrated overall $15 \%$ conversion efficiency is highest to date in an all-OPA-based CPA system pumped by a tabletop pump laser. $40 \%$ conversion should be possible with planned optimization of the multipass amplifier. With the optimized compression, transform-limited $30 \mathrm{fs}$ pulses should result, with a peak power of $2 \mathrm{TW}$ at a $10 \mathrm{~Hz}$ repetition rate. Scaling of this concept using all the energy 
from a typical 1.5-J pump laser should result in $10 \mathrm{TW}$ pulses, in a simple, all-passive, and relatively inexpensive device. Focusable intensity of such source should exceed $10^{18}$ $\mathrm{W} / \mathrm{cm}^{2}$. It is expected that this concept will find its use in university laboratories and industry due to its superior performance compared to a system based on OPCPA or Ti:sapphire alone. 


\section{Chapter 7}

\section{High average power optical parametric}

\section{chirped pulse amplification}

A particularly attractive and frequently overlooked feature of optical parametric amplification is the absence of intrinsic heat load in the nonlinear frequency conversion process. In conventional gain materials based on a laser transition, heat generation is a consequence of three factors: energy gap between the pump level and the fluorescence transition level leads to nonradiative phonon transitions; quantum efficiency of fluorescence is less than $100 \%$; and, normal material absorption occurs in laser material. ${ }^{88}$ Optical parametric amplification is, on the other hand, a "perfectly elastic" process, in which energy is conserved in the optical field. The difference between the energy levels of pump and signal photons is channeled to the third optical wave - idler (Fig. 7.1). This characteristic of OPA is sometimes viewed as the conservation of the photon number, and it is expressed by Manley-Rowe relations ${ }^{18}(2.46)$.

With the absence of the quantum defect and with the quantum efficiency of $100 \%$ to signal and idler, the only heat load that remains in OPA comes from material absorption due to the imaginary part of the dielectric susceptibility. It is therefore of foremost importance to identify the materials which, along with their favorable nonlinear properties, exhibit high transparency for all wavelengths involved in the process. 


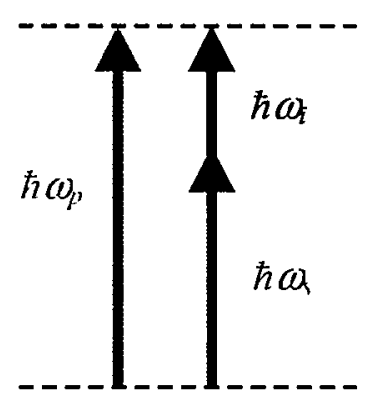

Figure 7.1. Optical parametric amplification is a perfectly elastic process in which the energy remains conserved in the optical field: $\omega_{3}+\omega_{i}=\omega_{p}$

In this Chapter, a numerical study is presented of the scaling of OPAs to high average power, with a particular attention to the enabling pump laser technology. Material choice is suggested by previous detailed studies of high average power nonlinear conversion. Heating effects in an OPA determine the maximum possible average power in a single OPA crystal. The model presented here is based on an iterative algorithm that leads to a self-consistent solution of nonlinear conversion equations and the thermal diffusion equation. Next, an architecture is presented that has a potential to increase the average power limit beyond that obtainable from a single OPA crystal. In the end, a design of the high average power pump laser is presented. Some preliminary experimental development of the next generation of high average power pump lasers for OPCPA is included. This effort should lead to the first OPCPA system with favorable characteristics for high-speed short-pulse materials processing. ${ }^{11}$ 


\subsection{Material selection}

Similar to laser gain media, it is important that nonlinear crystals exhibit favorable thermo-mechanical properties. In addition, thermally induced dephasing represents an important consideration in selection of thermally loaded nonlinear crystals. $\beta$-barium borate $(\mathrm{BBO})$ has been identified earlier as the material with an exceptional promise for high average power nonlinear optics applications. ${ }^{22,89-91}$ The absorption coefficients in $\mathrm{BBO}$ have been measured to be $<0.2 \% \mathrm{~cm}^{-1}$ for $1064 \mathrm{~nm}$ wavelength, ${ }^{92}$ and $<1 \% \mathrm{~cm}^{-1}$ for $532 \mathrm{~nm} .{ }^{93}$ It is noteworthy that the absorption in BBO is due entirely to the presence of inclusions, and it is expected that the absorption can be reduced through improvements in crystal growth. In the calculations presented here, conservative values for absorption coefficients given above are used.

$\mathrm{BBO}$ is a negative uniaxial crystal, with a broad transparency range $(0.198-2.6 \mu \mathrm{m}$ at $50 \%$ level). ${ }^{94}$ Temperature-induced wave vector mismatch calculations (Figs. 3.8-3.9) indicate a particularly broad temperature bandwidth in $\mathrm{BBO}$, allowing relatively large temperature gradients. When considering BBO OPAs, one of the limiting factors is the narrow angular tolerance for beams with extraordinary polarization, leading to the requirement for good collimation and beam quality of the pump laser (Figs. 3.10-3.11). In high average power applications, thermal load leads to a temperature distribution in the crystal. Refractive index gradient is then a result of the temperature-dependent refractive index and the stress distribution through stress-optic coefficients. As a result, thermal lens arises in the gradium-index medium and modifies the beam diameter and divergence as it propagates through the crystal. A small change of beam diameter does not severely 
influence conversion efficiency of OPA working in the regime of strong pump depletion. The beam divergence influences phase matching more severely. While the model presented in section 7.2 does not include this effect, it is important to address this issue experimentally as high average power pump lasers suitable for pumping OPCPA become available. The stress-optic coefficients of $\mathrm{BBO}$ are not available to date, and their measurement will be necessary to do more complete studies of high average power devices based on $\mathrm{BBO}$. Since $\mathrm{BBO}$ is a birefringent crystal, depolarization effects are weak and they are not expected to contribute significantly to dephasing.

On of the great difficulties in assessing the high average power performance in $\mathrm{BBO}$ is the lack of consistent set of data for thermal conductivity. In Table 7.1, the available published data for thermal conductivity in two principal directions is given.

\begin{tabular}{|c|c|c|}
\hline Reference & $K_{\|}\left(W \mathrm{~cm}^{-1} K^{-1}\right)$ & $K_{\perp}\left(W \mathrm{~cm}^{-1} K^{-1}\right)$ \\
\hline D. Eimerl et al. $(1987)^{22}$ & 0.008 & 0.0008 \\
\hline J. Beasley $(1994)^{95}$ & 0.016 & 0.012 \\
\hline
\end{tabular}

Table 7.1. Thermal conductity in BBO. $K_{\|}$and $K_{\perp}$ are the conductivities parallel and perpendicular to the crystal $\hat{z}$ axis, respectively.

While both sets of data show anisotropy, the extent of anisotropy and the general magnitude of coefficients vary greatly. While the more recent data ${ }^{95}$ is considered more reliable, ${ }^{96}$ a conservative approach is taken, and both sets of coefficients are used in calculations. The two sources are referenced appropriately when they are used. 


\subsection{Modeling of average power limits}

A model was developed which can address the conversion in OPA in the regime of high average power. The system of coupled wave equations $(2.33-2.35)$ can be rewritten to include absorption:

$$
\begin{aligned}
& \frac{d A_{1}(z)}{d z}=-\frac{\alpha_{1}}{2} A_{1}+i \frac{2 d_{e f f} \omega_{1}^{2}}{c^{2} k_{1}} A_{2}^{*}(z) A_{3}(z) \exp (i \Delta k z), \\
& \frac{d A_{2}(z)}{d z}=-\frac{\alpha_{2}}{2} A_{2}+i \frac{2 d_{e f f} \omega_{2}^{2}}{c^{2} k_{2}} A_{1}^{*}(z) A_{3}(z) \exp (i \Delta k z), \\
& \frac{d A_{3}(z)}{d z}=-\frac{\alpha_{3}}{2} A_{3}+i \frac{2 d_{e f f} \omega_{3}^{2}}{c^{2} k_{3}} A_{1}(z) A_{2}(z) \exp (-i \Delta k z) .
\end{aligned}
$$

Here, $\alpha_{1}, \alpha_{2}$, and $\alpha_{3}$ represent the absorption coefficients at the signal, idler and pump wavelength, respectively. Absorption coefficients are available for most optical nonlinear materials and represent a trivial addition to the model in the conditions of low average power. However, when the average power is increased, nonlinear conversion and thermal load have a more complicated relationship, particularly when the absorption is different for different wavelengths. In that case, a self-consistent numerical analysis of the optical conversion and thermal effects has to be performed.

The 3-dimensional method of high average power analysis performed in this work is illustrated in Fig. 7.2. The calculation starts with an initial guess of the temperature distribution $T(x, y, z)$ in the crystal. Optimal crystal orientation is determined from the temperature distribution by requiring that a zero phase mismatch occurs in the crystal center, where the expected conversion efficiency is maximum: 


$$
\int_{0}^{z} \Delta k\left(\theta_{o p t}, T(X / 2, Y / 2, z)\right) d z=0 .
$$

Here, the wave vector mismatch $\Delta k$ is a function of the optimal phase matching angle $\theta_{o p t}$ and the temperature of the crystal along the $\hat{z}$ axis. The dimensions of the crystal are $(X, Y, Z)$. By ensuring a zero phase mismatch in the crystal center, beam quality can be improved at the expense of the overall conversion efficiency. This optimization is an improvement over previously developed numerical methods. ${ }^{97}$ The wave vector mismatch as a function of temperature is determined via the temperature-dependent dispersion relations $\mathrm{s}^{22}$ for two principal polarizations $\left(n_{o}\right.$ and $\left.n_{e}\right)$ in $\mathrm{BBO}$ :

$$
\begin{aligned}
& n_{o}(\lambda, T)=\sqrt{2.7405+\frac{0.0184}{\lambda^{2}-0.0179}-0.0155 \lambda^{2}}\left(1-16.6 \times 10^{-6}(T-20)\right) \\
& n_{e}(\lambda, T)=\sqrt{2.3730+\frac{0.0128}{\lambda^{2}-0.0156}-0.0044 \lambda^{2}}\left(1-9.3 \times 10^{-6}(T-20)\right)
\end{aligned}
$$

In the next step, dephasing is calculated for every point in the crystal oriented at the optimal phase matching angle $\theta_{o p t}$.

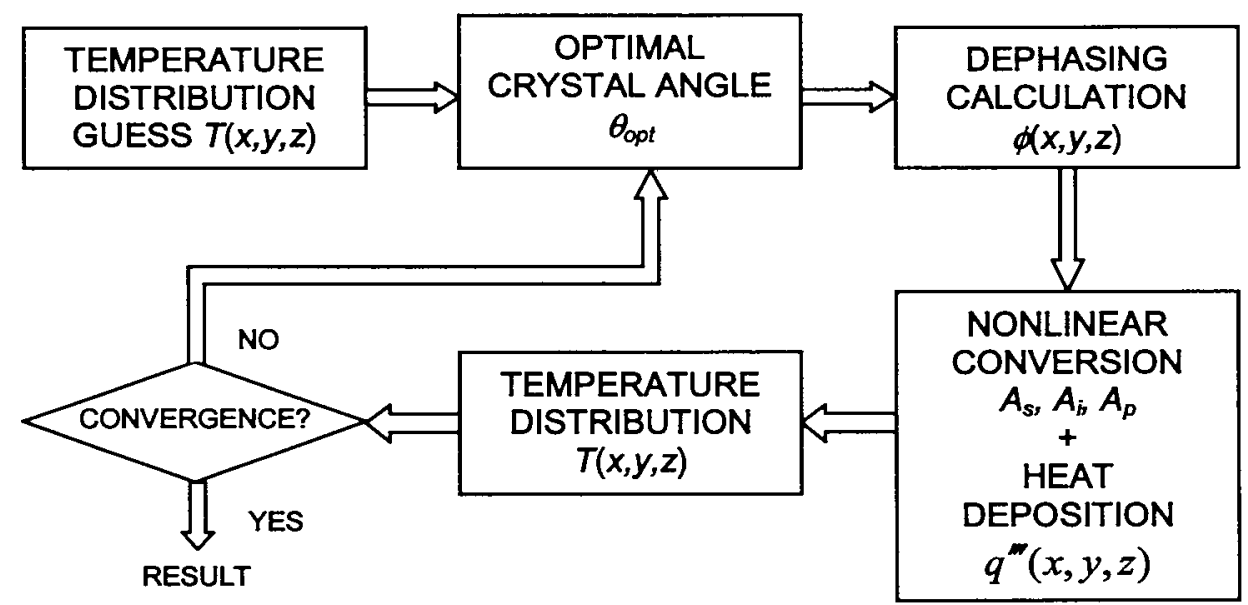

Figure 7.2. Coupled iterative nonlinear conversion - thermal diffusion method for calculation of the self-consistent, steady-state solution of the high average power OPA problem 
Boundary conditions for thermal analysis are the temperatures of the four lateral crystal faces and the requirement for zero heat flow across the front and the back crystal face. This approximates the usual cooling design, with the crystal faces exposed to air, and the crystal surrounded by a heat sink on the remaining four sides. A calculation is performed in a usual way to determine the conversion to signal, with absorption effects included following Eqs. (7.1)-(7.3). Heat deposition in the crystal is also determined by solvings Eqs. (7.1)-(7.3), which is then used to evaluate the steady-state temperature distribution. The temperature distribution in the crystal can be calculated by solving the thermal diffusion equation:

$$
K_{x} \frac{\partial^{2} T(x, y, z)}{\partial x^{2}}+K_{y} \frac{\partial^{2} T(x, y, z)}{\partial y^{2}}+K_{z} \frac{\partial^{2} T(x, y, z)}{\partial z^{2}}=q^{\prime \prime}(x, y, z),
$$

where $K_{x}, K_{y}$ and $K_{z}$ are the thermal conduction coefficients in the principal directions in the lab frame, and $q^{m}$ is the deposited heat power per unit volume at the same point. The temperature distribution is calculated from (7.7) by the iterative Gauss-Seidel method.

At this point, the calculation returns to the readjustment of the optimum crystal angle, followed by the conversion and heat deposition calculations with the newly calculated temperature distribution. Self-consistency of the coupled nonlinear conversion - thermal diffusion calculation is achieved when the difference between two consecutive solutions for conversion efficiency becomes negligible.

As an example of the calculation performed with the developed model, consider a $8 \times 8 \times 10 \mathrm{~mm}^{3}$ BBO crystal used in a type I process $1054 \mathrm{~nm}(0)+1074 \mathrm{~nm}(0)=532 \mathrm{~nm}$ (e). The pump energy is $100 \mathrm{~mJ}$, with a variable repetition rate $(0-50 \mathrm{kHz})$. Crystal lateral surfaces are kept at a temperature of $20^{\circ} \mathrm{C}$. The mesh size used in the calculation is $20 \times 20 \times 50$ points. Conversion efficiency degradation as a function of repetition rate is 
presented in Fig. 7.3. The calculation is performed for two sets of thermal conductivities from Table 7.1. While there is very little influence of the average power load below 200 W $(2 \mathrm{kHz})$, the reduction of conversion efficiency at a pump power of $400 \mathrm{~W}(4 \mathrm{kHz})$ approaches $50 \%$ (conductivity Ref. 22). If a newer set of conductivities (Ref. 95) is used, the conversion efficiency drops to $50 \%$ at a pump level $>1 \mathrm{~kW}$. In Fig. 7.4 (b), the resulting near-field signal profile at a pump power level of $500 \mathrm{~W}$ and with conductivities from Ref. 22 is presented, indicating a reduction of the signal beam diameter. This is an indication of poor conversion far from the phase-matched beam axis. The thermal

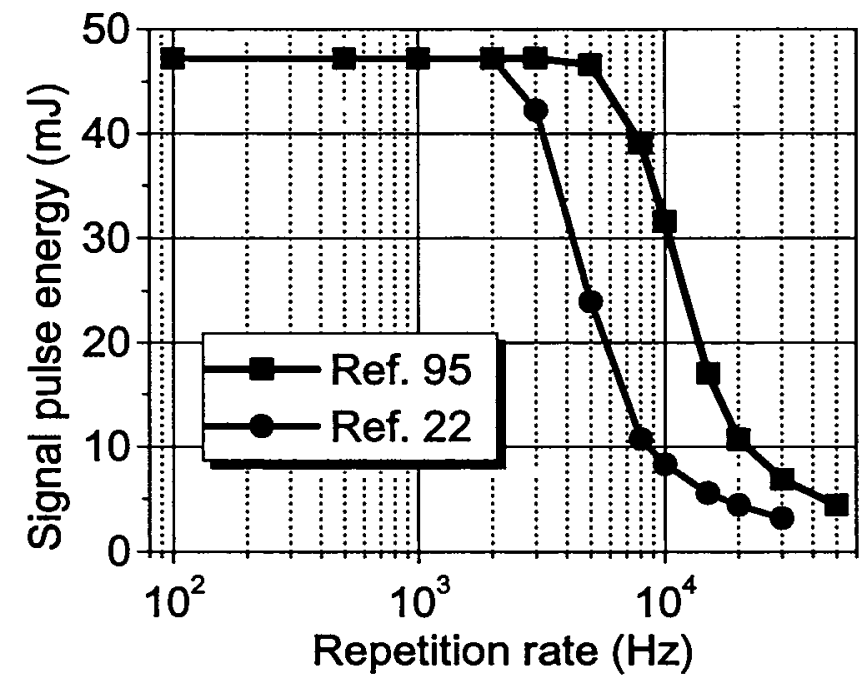

Figure 7.3. Signal pulse energy as a function of repetition rate in a high average power BBO OPA, for two different sets of thermal conductivities. Conservative average power calculations can be based on Ref. 22 .

deposition and temperature profile for the two cases is also shown in Fig. 7.4.

These results indicate that the maximum possible pump energy that can be passed through a $8 \times 8 \times 10 \mathrm{~mm}^{3} \mathrm{BBO}$ crystal with a negligible effect of the conversion efficiency and beam quality is near $200 \mathrm{~W}$ and $500 \mathrm{~W}$ in our configuration, with thermal 
conductivities from Refs. 22 and 95, respectively. This represents a conservative estimate since special crystal geometry and thermal removal schemes can be engineered for a specific problem.
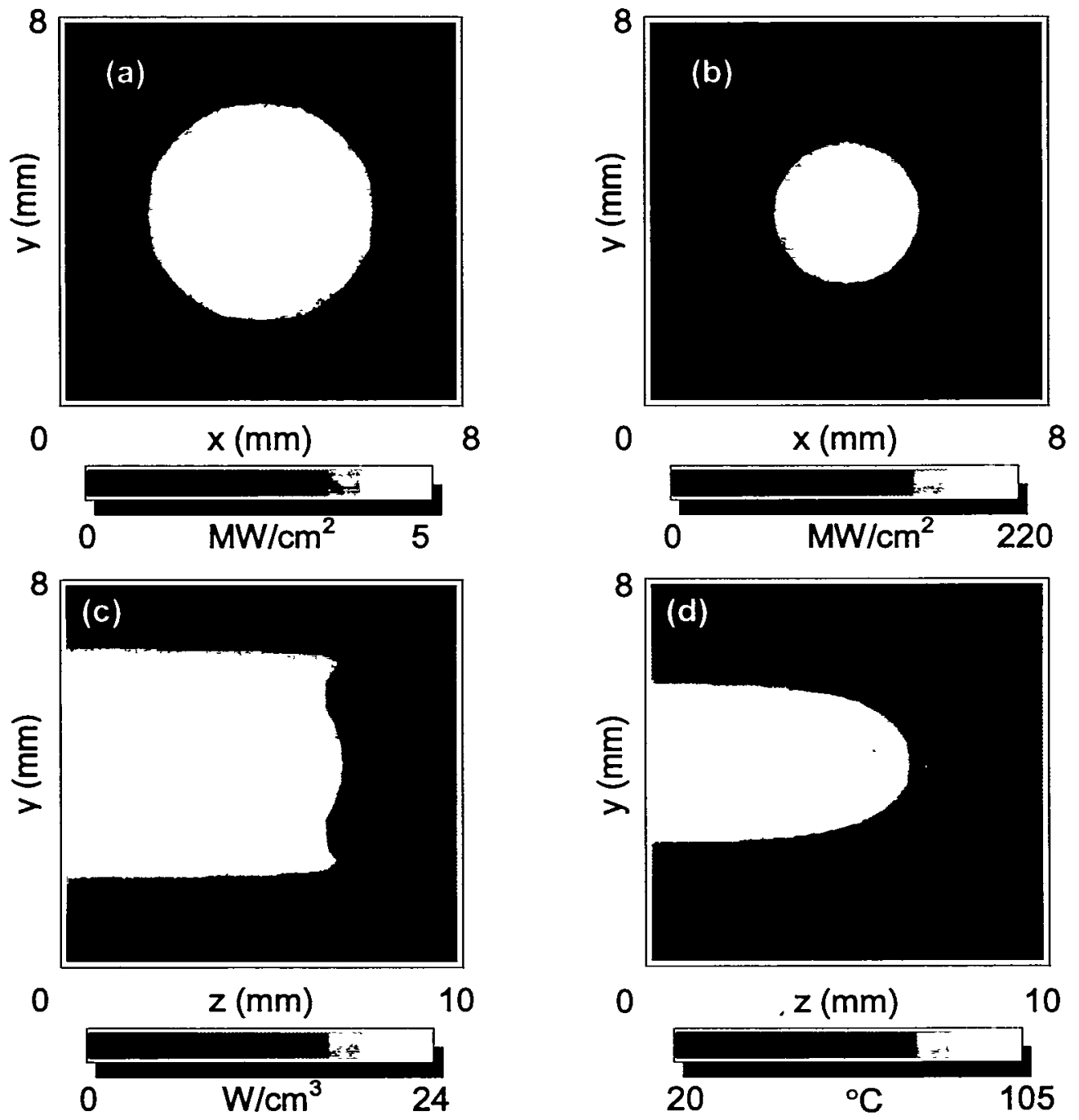

Figure 7.4. An example of the result of the calculation performed at a repetition rate of $5 \mathrm{kHz}$, with the set of thermal conductivity coefficients from Ref. 22 . The size of the seed beam (a) is $5.9 \mathrm{~mm}$. The beam size after amplification (b) is reduced to 3.9 $\mathrm{mm}$. The deposited thermal power in the central YZ plane of the crystal is shown in (c). Stronger absorption can be noted on the front of the crystal, where the pump beam is not depleted by interaction. The resulting steady-state thermal profile in the crystal is shown in (d). 
It important to note that this model does not include any stress-optic effects and does not predict the thermally induced mechanical failure. A previous study ${ }^{89}$ indicated that the fracture temperature of $\mathrm{BBO}$ is 10 times greater than other comparable nonlinear materials, with an anticipated fracture toughness of $150 \mathrm{kPa} \mathrm{m} \mathrm{m}^{1 / 2}$. Preliminary experimental tests of thermally induced fracture in BBO showed that about $300 \mathrm{~W}$ of 532 nm light did not lead to BBO fracture. ${ }^{98}$

\subsection{Cascaded difference-frequency generation for high average power}

In this section, a new scalable architecture for OPA is presented. It allows higher average power signal output than would be possible in a single crystal as a result of thermal effects induced by pump absorption. The average power limit of this architecture is set essentially by the thermal load induced by signal and idler absorption.

An important feature of normal dispersion is the fact that the absorption of shorter wavelength light is stronger than the absorption of longer wavelength light. Since the absorption in OPA is dominated by the shorter wavelength (pump), a multi-crystal scheme is proposed which limits the heat load through each crystal by splitting the incident pump power. Let us assume that the average power limit in a particular OPA at the pump wavelength is $300 \mathrm{~W}$, while the same limit at the signal/idler wavelength is 1500 W. As shown in Fig. 7.5, the last OPA crystal can be subjected to the maximum

allowed pump power $(299 \mathrm{~W})$ for assumed seed input of $5 \mathrm{~W}$ without thermal crystal degradation and/or crystal fracture, and with the acceptable level of reduction in nonlinear conversion. Assuming 30\% conversion efficiency (a conservative and 
experimentally confirmed value in OPAs ${ }^{81,82}$ ), the maximum signal output from this type of system would not exceed $100 \mathrm{~W}$.

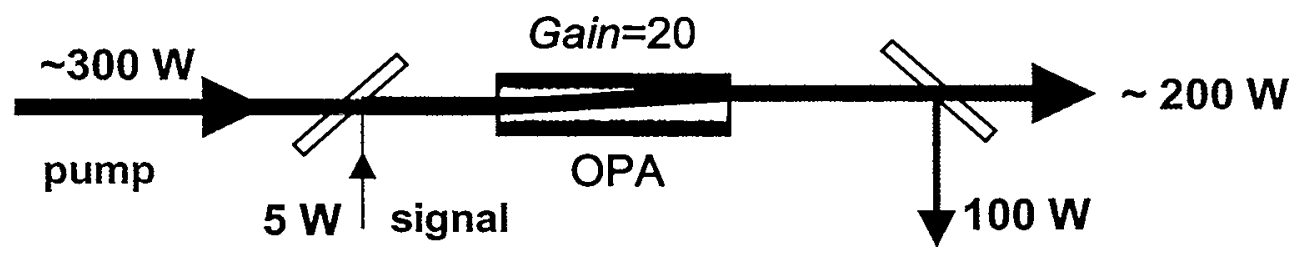

Figure 7.5. Final OPA crystal in a high average power system

Being limited by the pump absorption, OPA design can be modified to allow distribution of more total pump power into several OPA crystals. Fig. 7.6 shows an example of such design, consisting of three OPA crystals pumped by a total of $840 \mathrm{~W}$ of pump power, but still within the limits for material fracture. $840 \mathrm{~W}$ pump is split by a series of beamsplitters into three $(300 \mathrm{~W}, 280 \mathrm{~W}$, and $260 \mathrm{~W})$ beams, which are used to pump three OPA crystals. We may note that instead of using a single $840 \mathrm{~W}$ pump beam, the three pump beams can be originating from more than one pump laser, relaxing the requirements on individual pump laser power. Cascaded OPA crystals in this configuration work in a large-signal regime (difference-frequency generators), adding $\sim 30 \%$ of the pump beam pulse energy to the signal beam even as the pulse energies of signal and pump become comparable. Idler beam is discarded after each crystal using angular separation, which allows the large spectral bandwidth to remain conserved.

The most notable advantage of this architecture is that there is no sacrifice in the total conversion efficiency, because the signal power represents a nearly fixed fraction of pump power in every stage. A disadvantage of this approach is the growth of system complexity as addition stages are added (Fig. 7.6 does not include associated beam relay 
optics). In this way, the average power of OPCPA can be scaled in any crystal if a combination of signal and idler wavelength with low absorption is used, since the effect of absorption of short wavelength pump beam is greatly reduced.

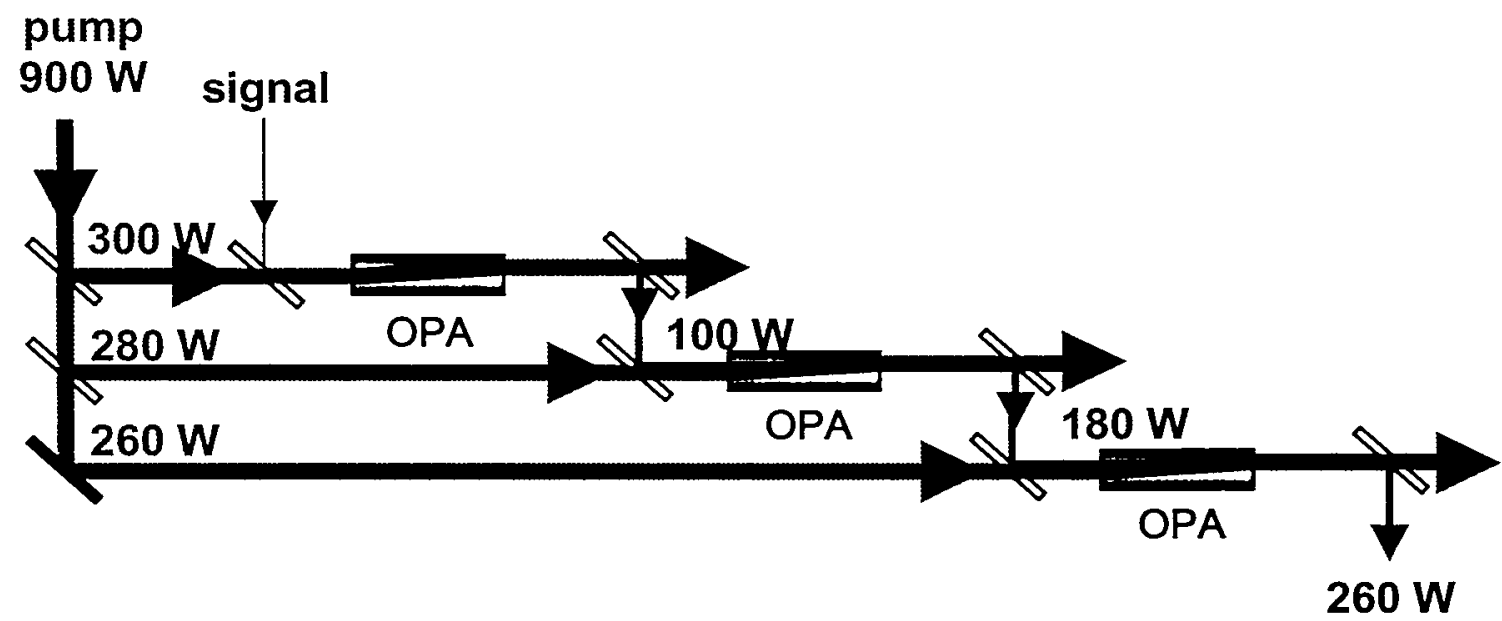

Figure 7.6. Distribution of heat load in OPA through cascaded differencefrequency generation. In the three-stage example system, the average power limit is increased by a factor of 2.6.

\subsection{High average power pump laser design}

A critical component of the high average power OPCPA system is the high average power pump laser. High average power OPCPA essentially separates the thermal load issues from the amplification process. The thermal issues are largely diverted from the short-pulse amplification material into the high average power nanosecond pump laser. The availability of narrow bandwidth laser gain materials with good thermal and mechanical properties is extensive, allowing the scaling of the pump laser and the entire OPCPA system to much higher average power than what can be obtained by using solely broadband laser amplification materials such as Ti:sapphire. 
yet that exhibits all the required characteristics simultaneously. This necessitates further development of the laser technology that will be able to accommodate the very stringent pump laser requirements for high-efficiency amplification in OPAs. In this section, a conceptual design and some preliminary results are presented for a high average power laser that can be used to pump OPCPA. While this is the first step in the direction of development of an appropriate pump laser, the final performance limits in OPCPA can be reached only by a more extensive development, with performance characteristics that are in many aspects several orders of magnitude beyond the best current technology. ${ }^{99,100}$

The proposed pump laser that will allow the development of the first multi-W, high repetition rate OPCPA in the near future is presented in Fig. 7.7.

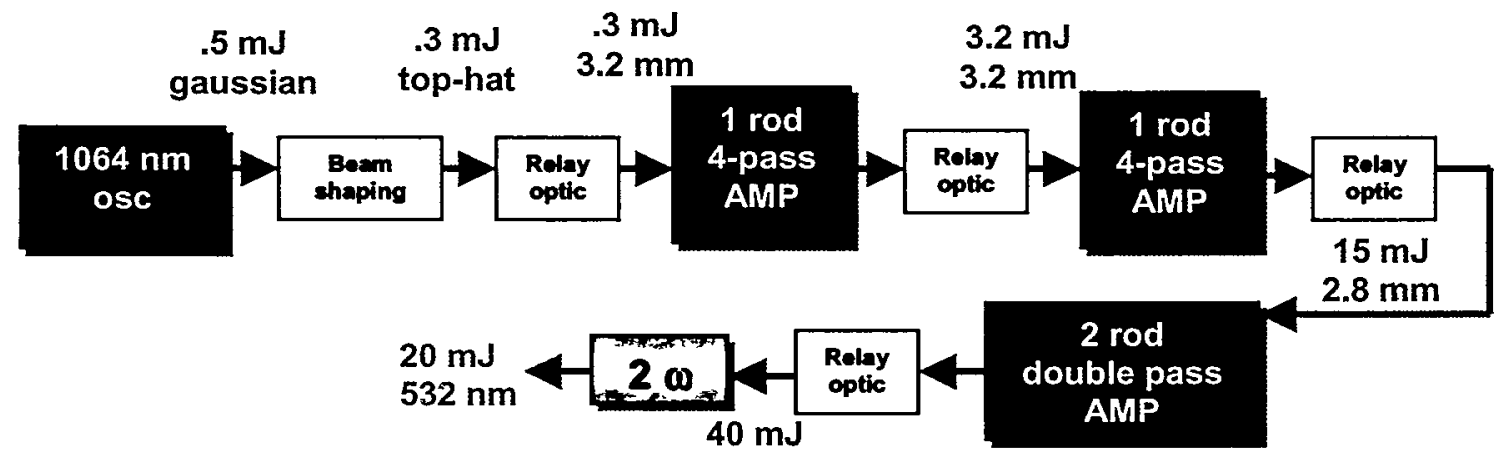

Figure 7.7. Design of a $80 \mathrm{~W}, 532 \mathrm{~nm}$ laser for pumping high average power OPCPA. Indicated are the pulse energy, transverse intensity profile and diameter of the beam at several points in the system.

The system is based on a master oscillator - power amplifier (MOPA) architecture. The master oscillator is a $1064 \mathrm{~nm}$ miniature Nd:YAG seeder laser, which produces $4 \mathrm{~ns}$ FWHM pulses at a $4 \mathrm{kHz}$ repetition rate. The seeder operates in single longitudinal mode to ensure a smooth temporal profile of the pump beam, and in TEM00 transverse mode to 
OPCPA imposes stiff requirements on the pump pulses, which is a result of the instantaneous energy transfer among coherent laser beams, as opposed to pump energy storage in conventional lasers. The instantaneous character of interaction indicates that matching the pump pulse width with the stretched signal pulse width is desirable. In this way, the extraction efficiency can be maximized, and the entire spectral bandwidth of the stretched signal pulse can be amplified. As an alternative, if the pump pulse is significantly longer than the signal pulse, temporal multiplexing can be employed to improve efficiency. As shown in Chapter 8, it is necessary that the pump laser produce a good beam quality to allow good conversion efficiency. This requirement is the consequence of the relatively narrow acceptance angle for the pump beam in critically phase-matched nonlinear crystals such as BBO. The pump laser has to operate with high repetition rate, which determines the overall repetition rate of the system. However, it is simultaneously important to produce a relatively high energy per pulse, as the OPA process can proceed efficiently only if the threshold peak power is exceeded. ${ }^{90}$ A low temporal jitter is required because of the instantaneous nature of the process. Additionally, constant space-time intensity of the pump pulse is desired (top-hat in space and time), which reduces the spatial and temporal (spectral) modification of the signal pulse. Finally, the pump pulse has to be at a shorter wavelength than the signal. This usually necessitates the use of frequency doublers, which leads to the loss of a significant fraction of the pump pulse energy at the fundamental frequency prior to its use in OPCPA.

Solid-state lasers have been demonstrated in the past that exhibit any one or a combination of the above required properties. However, no laser has been demonstrated 
maximize the beam quality. The seeder laser is described in more detail in the following section. The beam shaping section consists of a serrated aperture ${ }^{101}$ and a telescope with a spatial filter. The purpose of the beam shaping section is to produce nearly top-hat transverse beam profile by clipping the wings of the pump beam, followed by filtering of high spatial frequencies. The use of spatially top-hat beams has two benefits: it allows a greater energy extraction from the power amplifiers, and it is favorable for frequency conversion in the doubling crystal and OPCPA. It is necessary to relay image the top-hat spatial profile from the serrated aperture throughout the laser system, which is shown as a chain of relay optics between individual amplifier stages.

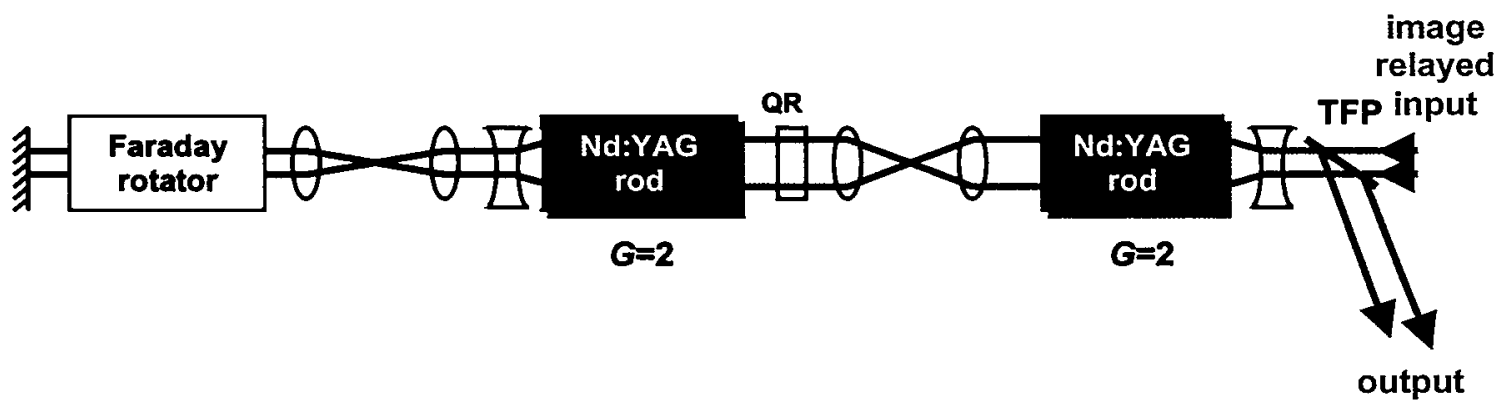

Figure 7.8. Design of the 2-rod double-pass amplifier. QR-quartz rotator, TFP-thin film polarizer. The gain of each rod is $G=2$.

The power amplifier consists of two 1-rod 4-pass Nd:YAG amplifiers and a 2-rod double-pass Nd:YAG amplifier. The amplifier heads are diode-pumped and made by Cutting Edge Optronics (CEO). They accommodate $50 \mathrm{~mm} \times 160 \mathrm{~mm}$ Nd:YAG laser rods, and allow extraction of up to $120 \mathrm{~W}$ of radiation from a single head when configured as a CW oscillator. ${ }^{102}$ The design of the 2-rod double-pass amplifier is presented in Fig. 7.8. Lenses are employed between different components to allow relay imaging. In addition to the preservation of the top-hat beam shape, relay imaging is necessary to compensate the thermal lens and thermally induced birefringence ${ }^{103,104}$ in 
individual amplifiers. Single-pass depolarization loss was measured to be $24 \%$ (Fig. 7.9 (a)), while the measured thermal lens of a single amplifier at full power was $160 \mathrm{~mm}$ (Fig. 7.9 (b)).
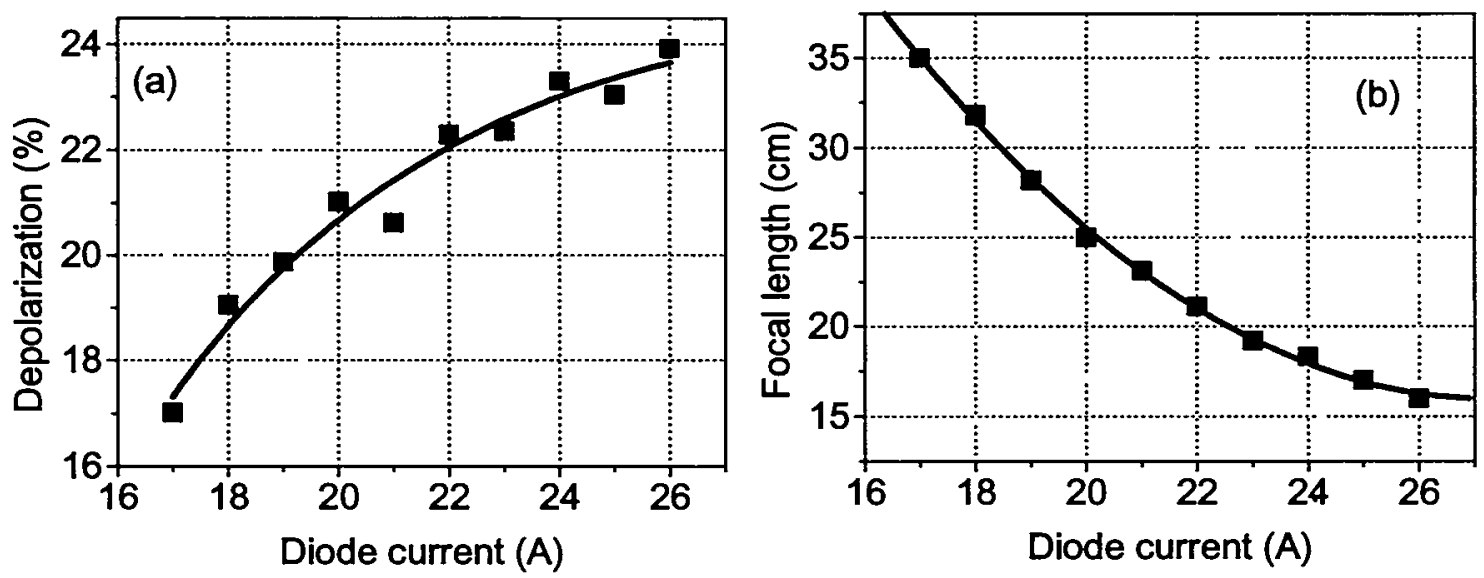

Figure 7.9. Single amplifier measurements for different diode current: (a) single pass depolarization, (b) thermal lens. Red lines represent polynomial fits.

Excellent depolarization compensation was obtained with a single-rod 4-pass amplifier, producing $<1 \%$ depolarization. This indicates a good mode matching and polarization rotation between individual passes of the amplifier. Depolarization loss of $8.5 \%$ was obtained by double passing the two-rod amplifier. The probable source of the depolarization correction imperfection in this case are the slightly different thermal properties and fluorescence distribution for two laser rods. Nevertheless, the obtained results indicate the possibility of construction of a Nd:YAG pump laser with a relatively good beam quality.

The amplifier is expected to produce $40 \mathrm{~mJ}$ pulses at a $4 \mathrm{kHz}$ repetition rate at the fundamental wavelength of $1064 \mathrm{~nm}$, with a top-hat transverse profile and a gaussian temporal profile. Doubling the fundamental wavelength is expected to produce $>20 \mathrm{~mJ}$ 
pulses. An addition of a fast temporal pulse slicer between the seeder laser and the first amplifier would be an improvement over the existing design, allowing higher conversion efficiencies in the final doubler and in the subsequent OPA.

\subsection{High repetition rate seeder laser}

The master oscillator section of the amplifier was developed as a miniature seeder laser. A schematic of the seeder laser is shown in Fig. 7.10. The gain medium is a Nd:YAG rod (1.5 mm x $10 \mathrm{~mm}, 0.7 \% \mathrm{Nd}$ doping). One side of the rod is coated for high reflection at $1064 \mathrm{~nm}$, representing one end of the cavity. Thin film polarizer allows only horizontal polarization to be amplified in the laser rod. A miniature, low-loss Pockels cell made of deuterated potassium dihydrogen phosphate $\left(\mathrm{KD}^{*} \mathrm{P}\right)$ allows quarter-wave switching at a voltage of $2.3 \mathrm{kV}$. The Pockels cell is followed by a half-waveplate and a flat $80 \%$ output coupler.

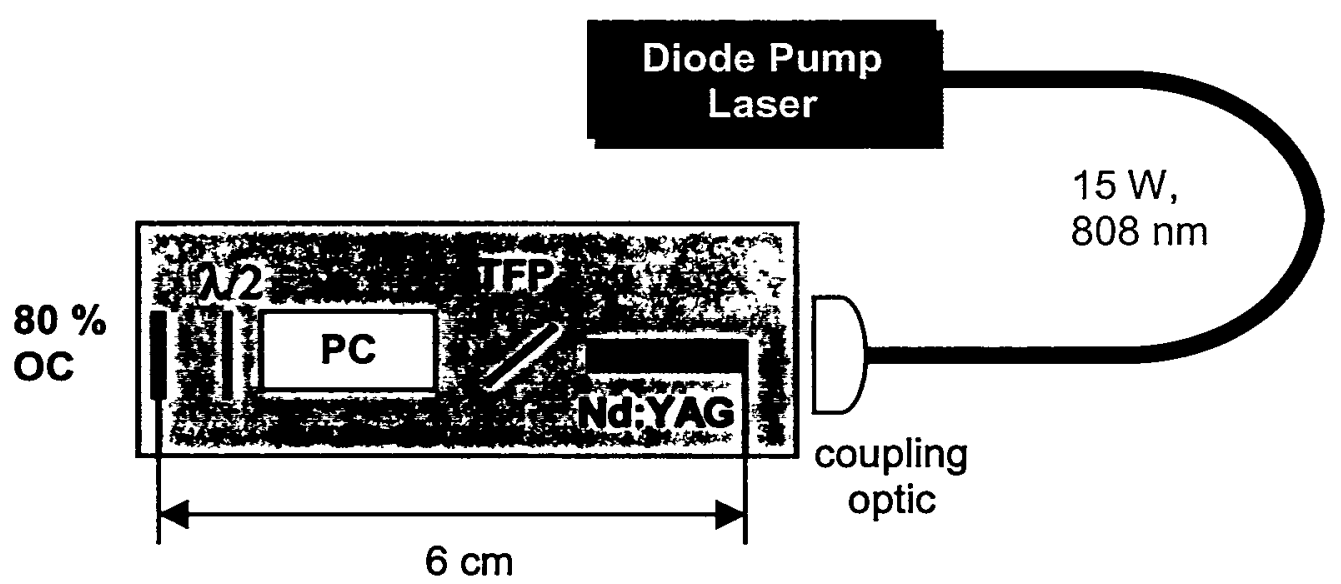

Figure 7.10. Seeder laser for high average power OPCPA pump laser. TFP - thin film polarizer, PC - Pockels cell, OC - output coupler. 
The laser was pumped by a fiber coupled diode laser $(808 \mathrm{~nm}, 15 \mathrm{~W})$, with a fiber core of $400 \mu \mathrm{m}$ and a numerical aperture of 0.13 . The pump beam was focused into the laser rod using a single lens. The small fiber core diameter and numerical aperture was essential for achieving a single transverse mode operation. Single longitudinal mode operation is also a result of short laser cavity (only $6 \mathrm{~cm}$ ), which introduces a large cavity mode separation and preferential selection of a single mode. The base of the laser is water-cooled (base), while the fiber coupled pump diode laser is air-cooled.

The obtained pulse width from the seeder laser was $4 \mathrm{~ns}$ at a repetition rate of up to $2 \mathrm{kHz}$. To verify that the laser was operating in a single longitudinal and a single transverse mode (TEM00), a Fabry-Perot interferometer was used. The maximum obtained power from the Q-switched laser was $1.2 \mathrm{~W}$ at a $3 \mathrm{kHz}$ repetition rate. The pulse energy decreased and the pulse width increased as the repetition rate was increased beyond $2 \mathrm{kHz}$. This is in good agreement with the fluorescence lifetime of Nd:YAG of $230 \mu \mathrm{s}^{88}$ Fig.7.11 shows the high repetition rate seeder laser.

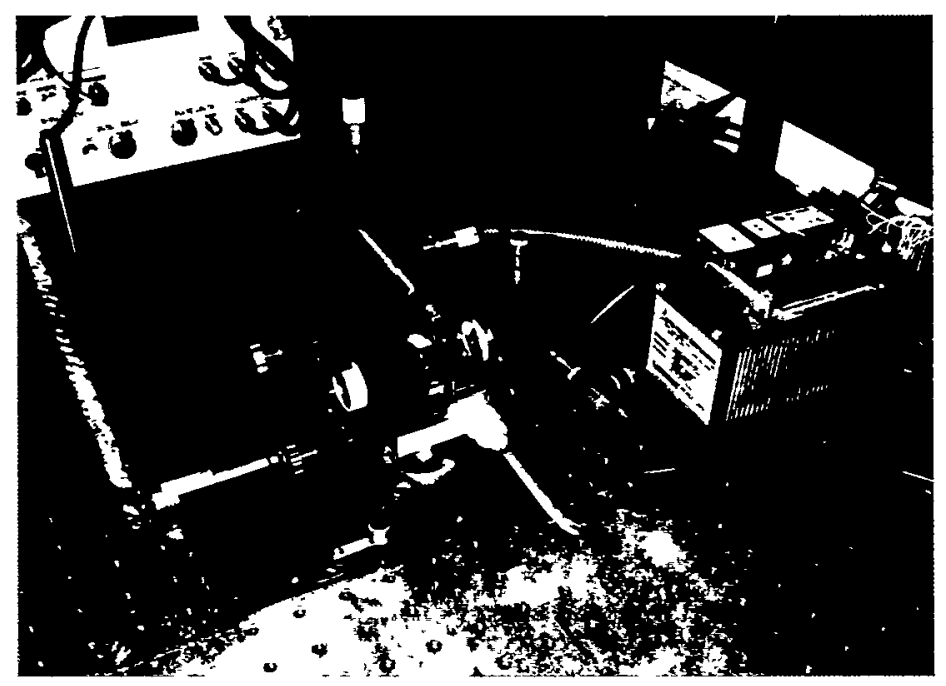

Figure 7.11. High repetition rate seeder laser 


\section{Chapter 8}

\section{Angular effects and beam quality in}

\section{optical parametric amplification}

Advances in optical parametric devices, in particular those requiring high conversion efficiency, rely on pump laser and gain medium properties. In this Chapter, a theoretical model is developed that describes the source of dephasing due to angular deviation from ideal phase matching in optical parametric amplification. Real laser beams have angular content, which is described by their spatial frequency spectrum. Such beams cannot be treated as single plane waves in nonlinear interactions. The mathematical model is based on a plane wave decomposition of gaussian and top-hat beams into their components in spatial frequencies. Several popular nonlinear materials (BBO, LBO, $\mathrm{KDP}$ ) are examined and the angular dephasing is rigorously calculated. The impact of the beam angular content on small signal gain and on conversion efficiency in the strongly depleted regime is evaluated numerically. In addition, a criterion is formulated for beam quality tolerance in OPAs, for critical and noncritical phase matching. The impact of initial conditions in optical parametric amplification is considered. The calculations presented in this chapter are primarily intended for devices pumped with long (nanosecond) pulses. The results should provide insight into the pump laser requirements for OPCPA. 
Careful maximization of efficiency is particularly important for high repetition rate OPAs, where the available pump pulse energy is relatively small. The conversion efficiency depends on the overlap and shape of pump and signal pulses in space and time. $^{81,82}$ When nanosecond pump pulses are used, longer interaction lengths in the crystals are required. Under these conditions, dephasing due to angular, spectral and temperature bandwidth and crystal nonuniformity becomes important. Boyd and Kleinman ${ }^{105}$ performed the first analytical calculations of coupled wave interactions with focused gaussian beams. Their calculations included effects of rapidly variable intensity in tight focusing conditions, walk-off and diffraction. However, their analysis was limited to small conversion efficiency and gaussian beams. More recently, Wong et al. ${ }^{106}$ investigated conversion efficiency for second-harmonic generation. In their model they used a beam with gaussian distribution of divergence and included depletion effects. Here, the calculation of Wong et al. is extended to predict the limits of performance for OPAs, ' using a plane wave decomposition model for gaussian and top-hat beams. A relationship between laser beam quality and conversion in OPAs is established. The impact of initial conditions in the process of optical parametric amplification is included in the model. Finally, a criterion is derived for beam quality requirements that allow large conversion efficiency in OPAs, for critical and noncritical phase matching.

\subsection{Preliminary considerations}

In optical parametric devices, nonlinear crystals are commonly placed in the laser beam waist, which allows the laser intensity to rise to the level required for efficient 
parametric interactions and ensure best possible collimation. A perfect gaussian beam propagates according to the following equation:

$$
w^{2}(z)=w_{0}^{2}\left(1+\left(\frac{\lambda\left(z-z_{0}\right)}{\pi w_{0}^{2}}\right)^{2}\right),
$$

where $w$ is the beam radius, $w_{0}$ is the beam waist radius, and $z$ is the longitudinal position ( $z_{0}$ being the waist position). In the beam waist, the one-dimensional distribution of the electric field in spatial frequencies can be obtained by taking the Fourier transform of the transverse electric field distribution:

$$
E(s)=\int_{-\infty}^{\infty} E(x) e^{-i s x} d x
$$

where $s$ is the transverse spatial frequency. The Fraunhofer diffraction pattern in the far field is equivalent to the near-field distribution in spatial frequencies $(s=\theta / \lambda)$. Therefore, any real laser beam consists of a superposition of plane waves, with a distribution of divergence as indicated by the spatial frequency distribution. This divergence spread contributes to dephasing in optical parametric amplification.

$\mathrm{M}^{2}$-formalism ${ }^{107}$ is used to describe beam propagation when transverse beam quality differs from the ideal transform limit:

$$
w^{2}(z)=w_{0}^{2}\left(1+\left(M^{2} \frac{\lambda\left(z-z_{0}\right)}{\pi w_{0}^{2}}\right)^{2}\right)
$$

Imperfect beam quality can be treated as an increase in the minimum beam waist - spatial frequency bandwidth product,

$$
w_{0} \sigma_{s 0}=\frac{M^{2}}{2 \pi},
$$


where the beam waist $w_{0}$ and spatial frequency bandwidth $\sigma_{s 0}$ are defined as variances of the beam intensity distribution in configuration and spatial frequency space, respectively. In this analysis two ideal beam profiles are considered: gaussian, a common beam profile obtainable from laser resonators, and top-hat, favored for nonlinear conversion processes due to reduced spatial variation of gain and reduced Poynting vector walk-off effects. While most real beams used in optical parametric amplification have beam profiles intermediate between the two ideal cases, this analysis still gives a quantitative assessment of the expected conversion from real beams.

Perfect gaussian beam intensity distribution can be written in terms of the electric field amplitude:

$$
E(r)=E_{0} \exp \left(-\frac{r^{2}}{w^{2}}\right)
$$

The corresponding distribution in spatial frequencies is obtained by taking the Fourier transform of (8.5):

$$
|E(s)|=\pi E_{0} w^{2} \exp \left(-\pi^{2} s^{2} w^{2}\right)
$$

or, for a beam that is not diffraction limited,

$$
|E(s)|=\frac{\pi E_{0} w^{2}}{M^{4}} \exp \left(-\frac{\pi^{2} s^{2} w^{2}}{M^{4}}\right) .
$$

A uniphase distribution is chosen for the pump: $E(s)=|E(s)|$. This arbitrary choice is justified by the phase insensitivity of difference-frequency generation. For every pair of angular components of the signal and pump, an idler is generated with the phase given by $\phi_{p}-\phi_{s}-\phi_{i}=-\pi / 2$. Therefore, any initial phase difference among the angular components is projected to the idler as idler phase content. 
Spatial frequency spectrum of the ideal top-hat beam can be calculated by taking the Hankel transform of the near-field electric field distribution:

$$
E(s)=E_{0} w^{2} \frac{J_{1}(2 s w)}{s w}
$$

where $J_{1}$ denotes the first order Bessel function. For a top-hat beam with a beam quality parameter $M^{2}$, the spatial frequency spectrum can be defined in analogy to the gaussian case as

$$
E(s)=\frac{E_{0} w^{2}}{M^{4}} \frac{J_{1}\left(2 \pi s w / M^{2}\right)}{s w / M^{2}}
$$

The minimum value of the angular divergence $\alpha$ corresponds to $M^{2}=1$.

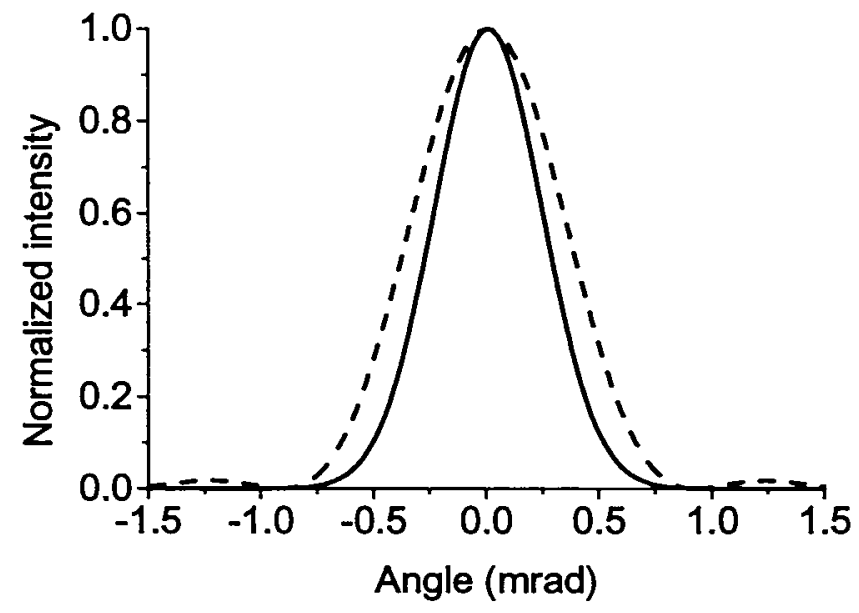

Figure 8.1. Normalized intensity distributions in divergence angles. The solid curve represents a gaussian and the dashed curve represents a top-hat beam; $w_{0}=0.5 \mathrm{~mm}, \lambda=1054 \mathrm{~nm}$.

Fig. 8.1 shows normalized intensity distributions in divergence angles for a gaussian TEM00 and a top-hat radially symmetric beam of equal radial intensity variance. Note that the spatial frequency spectrum for a top-hat beam is broader than for the gaussian beam for the same radial intensity variance. While the spatial frequency 
variance cannot be evaluated for a top-hat beam, ${ }^{107}$ the calculated distribution for top-hat beam nevertheless allows quantitative analysis in OPAs. An OPA acts as a spatial filter on the spatial frequency distributions (8.7) and (8.9) due to its finite angular phase matching bandwidth. This limits the spatial frequency range that needs to be evaluated. In the test cases presented here, the relevant spatial frequencies are limited to $3 \sigma_{\mathrm{s} 0}$ for gaussian beams, and to the secondary zero of the intensity distribution in spatial frequencies for top-hat beams.

When the beam is not propagating along one of the crystal axes, the change of the index of refraction is more sensitive to angular deviation in the principal plane of the crystal (critical phase matching). As previous calculations in Chapter 3 indicate (Fig. 3.11), detuning in the principal plane is the dominant contribution to total angular detuning. A general two-dimensional analysis for uniaxial crystals can therefore be reduced to a computationally less demanding one-dimensional case, where only detuning in the principal plane is considered. In biaxial crystals, critical phase matching is generally achieved in one of the principal planes of the crystal. In that case, the same analysis can be applied to biaxial crystals, taking advantage of the large difference in angular sensitivity in the principal plane and perpendicular to the principal plane. The one-dimensional field distribution is calculated as

$$
\left|E_{1}\left(s_{x}\right)^{2}=C \int_{-\infty}^{\infty}\right| E\left(\left.\sqrt{s_{x}^{2}+s_{y}^{2}}\right|^{2} d s_{y}\right.
$$

where $s_{x}$ and $s_{y}$ are the spatial frequencies in two principal directions, $E_{1}\left(s_{x}\right)$ is the onedimensional field distribution, $E(s)$ is the radial field distribution in spatial frequencies, and $C$ is the normalization constant, such that 


$$
\int_{-\infty}^{\infty}\left|E_{1}\left(s_{x}\right)\right|^{2} d s_{x}=\int_{0}^{\infty}|E(s)|^{2} 2 \pi s d s .
$$

A notable exception to this simplified treatment is the analysis of noncritically phase-matched processes, where beam propagation is achieved along one of the principal axes. In that case, angular sensitivity has similar magnitude in both directions, requiring a full two-dimensional analysis. A common example for a noncritically phase-matched crystal is lithium triborate (LBO), with its sensitivity curve plotted in Fig. 3.10 (d).

\subsection{Model formulation}

This study focuses on dephasing due to angular deviation from the ideal phase matching in optical parametric amplification. For this model a single frequency pump and signal and negligible group velocity dispersion are assumed. Without parametric interaction, the transverse pump beam intensity distribution is assumed constant along the length of the crystal $z$. The parametric interaction is evaluated at an arbitrary transverse point $(x, y)$ on the crystal. The calculation is performed from $\left(x, y, z_{i}\right)$ to $\left(x, y, z_{f}\right)$, where $z_{i}$ is at the input face and $z_{f}$ is at the exit face of the crystal. Aside from depletion effects, the initial angular distribution of the pump chosen at $z_{i}$ is maintained throughout the length of the crystal. The spatial frequency distribution of the electric field is used as the angular distribution for the plane wave decomposition at the input face of the crystal. Intensity variations are neglected, as the focus of interest are nanosecond OPAs, with relatively weak focusing and long depth of focus compared to the OPA length. Also, the Guoy 
phase shift that occurs on relatively short scale when tightly focused beams are used is not relevant to this calculation with weakly focused beams and short crystals.
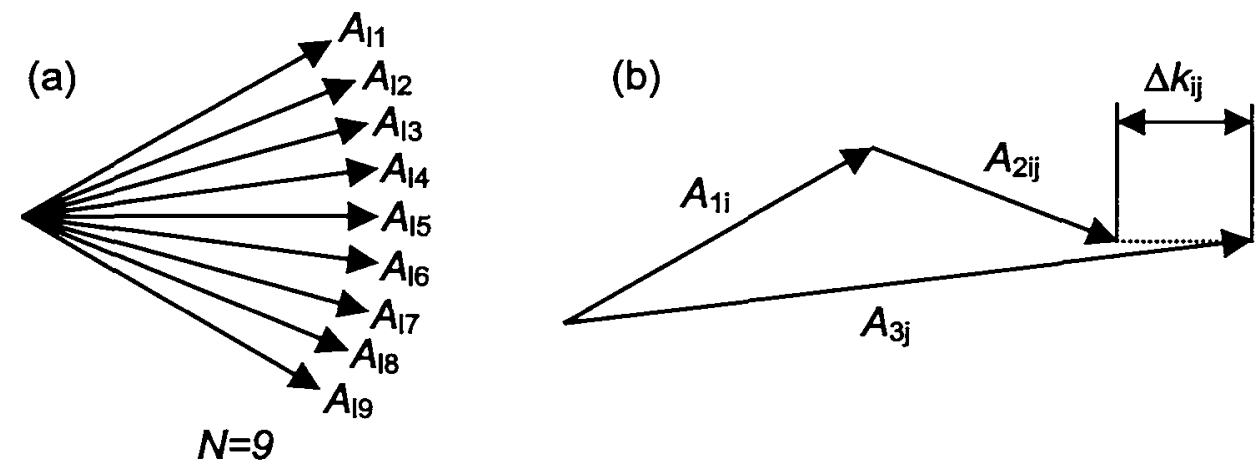

Figure 8.2. (a) Angular plane wave decomposition of a real laser beam, (b) assignment of the idler $A_{2 \mathrm{jj}}$ and wave vector mismatch $\Delta k_{i j}$ for the signal component $A_{1 \mathrm{i}}$ and pump component $A_{3 \mathrm{j}}$.

The electric field for signal and idler wave is represented as a sum of plane waves with angular distribution identical to the one-dimensional electric field distribution in angles (8.10):

$$
\mathbf{E}_{l}=\sum_{i=1}^{N} A_{l i} \mathbf{e}_{n} \exp \left[-i\left(\omega_{l} t-\mathbf{k}_{l i} \mathbf{r}\right)\right], \quad l=1,3
$$

where $e_{\mathrm{li}}$ represents the unit vector perpendicular to the propagation direction of the corresponding plane wave, and $A_{l i}$ represents the amplitude of an individual plane wave, normalized to the intensity $I_{l}$ of the corresponding beam:

$$
\sum_{i=1}^{N} A_{l i}^{2}=\frac{\mu_{0}}{\varepsilon_{0}} \frac{I_{l}}{2 n_{l}}, \quad l=1,3
$$

The limited width of angles about the phase matching angle for the signal and pump is uniformly divided into $N$ angular components, as shown in Fig. 8.2. A finite angular 
range is used for signal and pump as described above. An approximation can be made: $\mathbf{k}_{\mathbf{l i}}$ $\mathbf{r}=k_{\mathrm{l}} z$, as a consequence of paraxial beam propagation. Coupled differential equations that govern difference-frequency generation can now be solved:

$$
\begin{gathered}
\frac{d A_{1 i}}{d z}=i \frac{2 \omega_{1} d_{e f f}}{n_{1} c} \sum_{j=1}^{N} A_{2 i j}^{*} A_{3 j} \exp \left(i \Delta k_{i j} z\right), i=1, \ldots N, \\
\frac{d A_{2 i j}}{d z}=i \frac{2 \omega_{2} d_{e f f}}{n_{2} c} A_{1 i} A_{3 j}^{*} \exp \left(i \Delta k_{i j} z\right), \quad i=1, \ldots N, j=1, \ldots, N, \\
\frac{d A_{3 j}}{d z}=i \frac{2 \omega_{3} d_{e f f}}{n_{3} c} \sum_{j=1}^{N} A_{1 i} A_{2 j} \exp \left(-i \Delta k_{i j} z\right), \quad j=1, \ldots N .
\end{gathered}
$$

where $k_{i j}$ is the wave vector mismatch among the signal angular component $A_{1 i}$, idler angular component $A_{2 i j}$, and pump angular component $A_{3 j}$. The wave vector mismatch $k_{i j}$ is given by (2.56) for uniaxial crystals, and by (2.52-2.63) for biaxial crystals. Note that an idler angular component $A_{2 i j}$ is assigned to each combination of the angular components of the signal $A_{1 i}$ and pump $A_{3 j}$. The direction of the generated idler angular component $A_{2 i j}$ is defined by the nonlinear Snell's law for the incident signal and pump angular components $A_{1 i}$ and $A_{3 j}$, respectively.

The system of coupled differential equations is solved by the 4th order RungeKutta method for numerical integration. The required number of the integration steps and the transverse mesh size are determined by testing the resultant convergence with increasing grid finesse. Typical calculations were carried out with $N=50$ in the spatial frequency domain and 1000 steps in the numerical integration per $\mathrm{cm}$ of crystal length. The analysis tracks the electric field amplitude and phase throughout the interaction region, followed by the calculation of total beam intensity and gain. 


\subsection{Results of model calculations}

The analysis now proceeds to calculate the influence of the angular divergence on small signal gain in an OPA. In order to quantify the beam divergence in these calculations, the pump beam divergence parameter $\alpha$ is introduced:

$$
\alpha=\frac{M^{2} \lambda}{w}
$$

The divergence parameter takes into account beam size, wavelength and beam quality, and is proportional to the far-field diffracted angle. The following simulation parameters are chosen: $I_{p}=500 \mathrm{MW} / \mathrm{cm}^{2}, I_{\text {seed }}=10^{-3} \mathrm{~W} / \mathrm{cm}^{2}, \lambda_{p}=532 \mathrm{~nm}$, and $\lambda_{s}=1054 \mathrm{~nm}$. The difference-frequency mixing process used is $1054 \mathrm{~nm}(0)+1074 \mathrm{~nm}(0)=532 \mathrm{~nm}$ (e) . The minimum radial intensity variance - spatial frequency bandwidth product is assumed for the incident seed beam. This corresponds to the experimentally relevant case of amplification of a high-quality seed beam using an energetic pump beam of less than ideal beam quality.
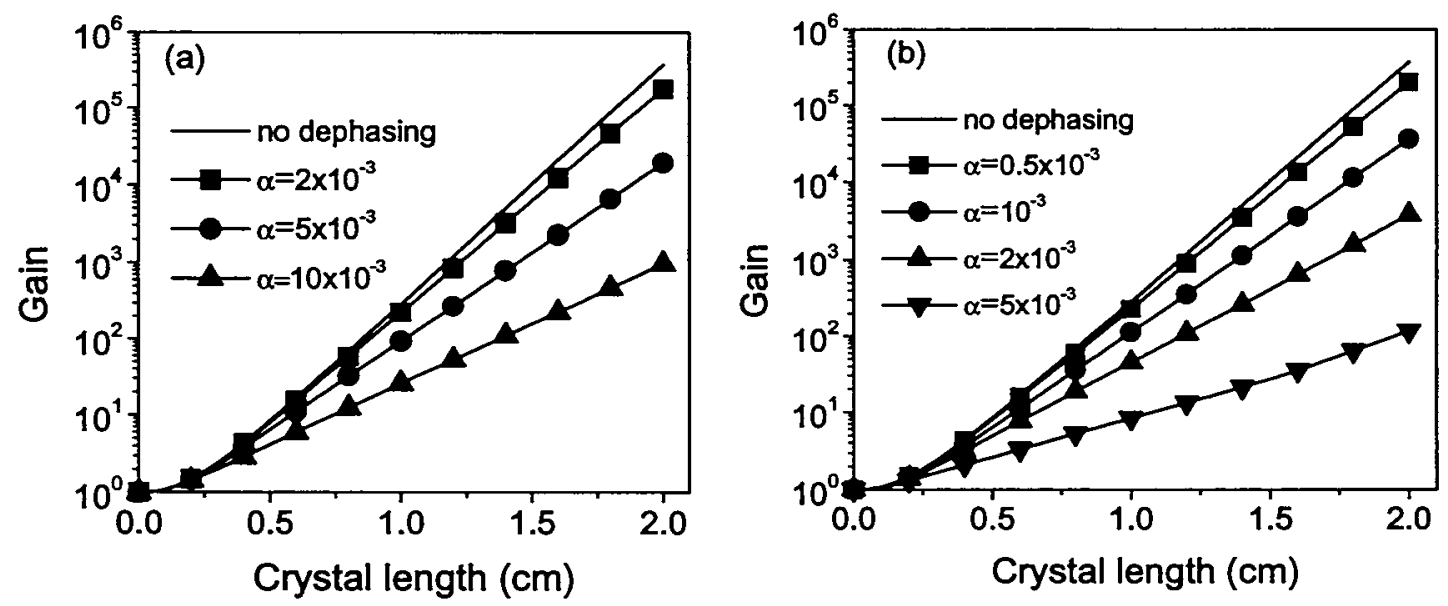

Figure 8.3. Small signal gain in BBO for different divergence parameters, for (a) gaussian beams, (b) top-hat beams. Simulation parameters: $\lambda_{s}=1054 \mathrm{~nm}, \lambda_{p}=532$ $\mathrm{nm}, I_{p}=500 \mathrm{MW} / \mathrm{cm}^{2}$. 

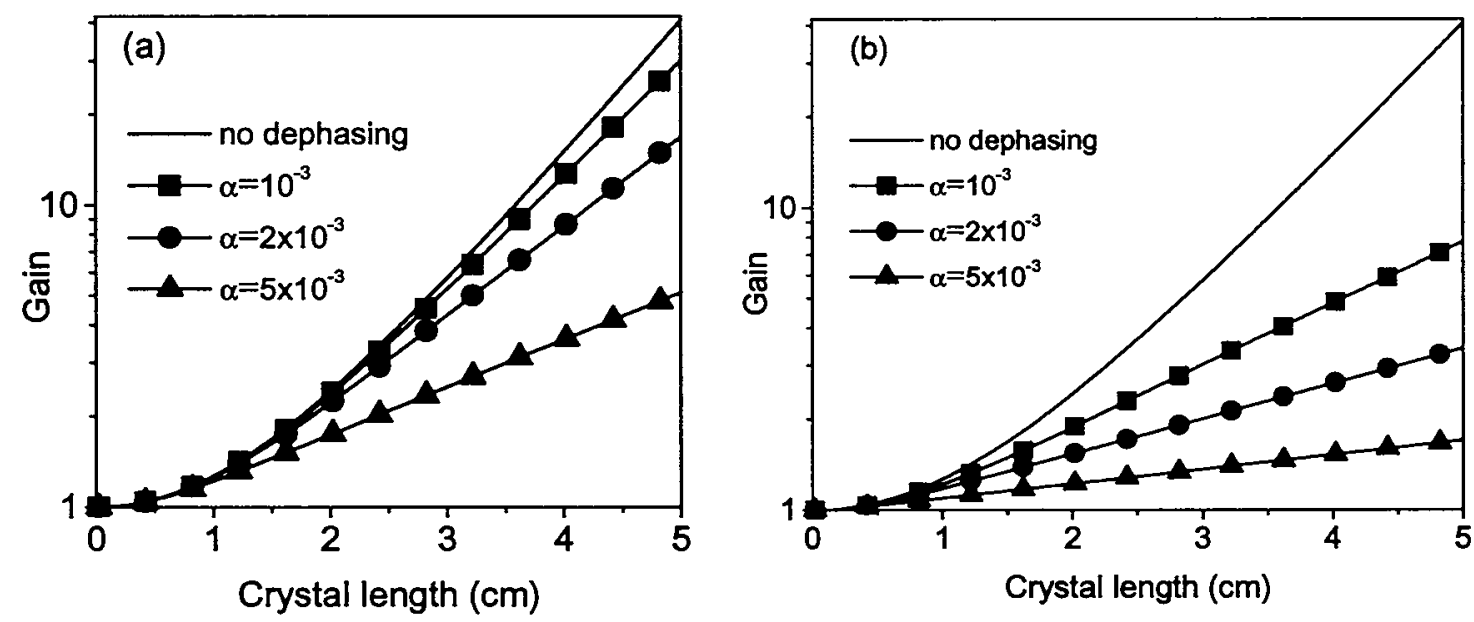

Figure 8.4. Small signal gain in KDP for different divergence parameters, for (a) gaussian beams, (b) top-hat beams. Simulation parameters: $\lambda_{s}=1054 \mathrm{~nm}, \lambda_{p}=532$ $\mathrm{nm}, I_{p}=500 \mathrm{MW} / \mathrm{cm}^{2}$.
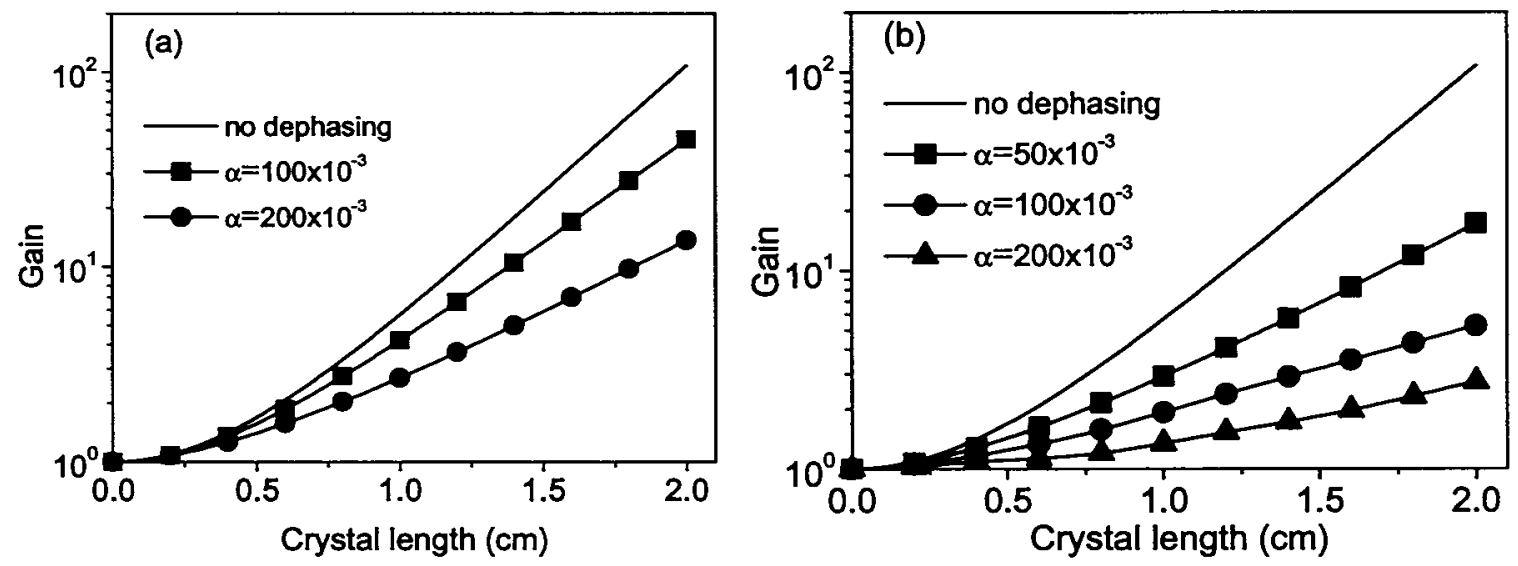

Figure 8.5. Small signal gain in noncritically phase-matched LBO for different divergence parameters, for (a) gaussian beams, (b) top-hat beams. Simulation parameters: $\lambda_{s}=1054 \mathrm{~nm}, \lambda_{p}=532 \mathrm{~nm}, I_{p}=500 \mathrm{MW} / \mathrm{cm}^{2}$. Note that the divergence parameter scale is an order of magnitude greater than for $\mathrm{BBO}$ and KDP.

Fig. 8.3(a) shows the small signal gain in $\beta$-barium borate $(\mathrm{BBO})$ as a function of interaction length in the crystal, for several divergence parameters $\alpha$ with gaussian beams. Indicated in Fig. 8.3(a) is the model calculation when zero dephasing $(\Delta k=0)$ is 
assumed. It is in agreement with a simple calculation based on a single, perfectly phasematched plane wave. An identical calculation is performed with a top-hat beam, with results shown in Fig. 8.3(b). It is apparent that a reduction of gain occurs even for modest values of divergence parameter $\alpha$, consistent with the relatively narrow angular acceptance of $\mathrm{BBO}$. It can be observed that the gain reduction is more severe for a tophat beam than for a gaussian beam with the same divergence parameter $\alpha$, resulting from the broader spatial frequency distribution for a top-hat beam compared to a gaussian beam with the same radial intensity variance $w$. Identical behavior is observed in potassium dihydrogen phosphate (KDP). This crystal is also a uniaxial crystal, but it has a much lower nonlinearity than BBO. KDP is available in large apertures and it is therefore attractive for high-energy nonlinear applications. Results are shown in Fig. 8.4, with the same interaction parameters, except that longer crystal length was observed due to relatively small gain. Finally, lithium triborate (LBO) is analyzed in a noncritically phase-matched configuration. Noncritical phase matching in LBO for the type I process $1054 \mathrm{~nm}(\mathrm{o})+1074 \mathrm{~nm}(\mathrm{o})=532 \mathrm{~nm}$ (e) can be achieved by heating the crystal to $\sim 150^{\circ}$ C. This configuration is characterized by large angular tolerance. The result of small signal gain calculations in noncritically phase-matched LBO is shown in Fig. 8.5. Note the same qualitative behavior for both noncritically phase-matched and critically phasematched crystals. Noncritically phase-matched LBO exhibits an order of magnitude greater divergence tolerance than $\mathrm{BBO}$ and $\mathrm{KDP}$. 

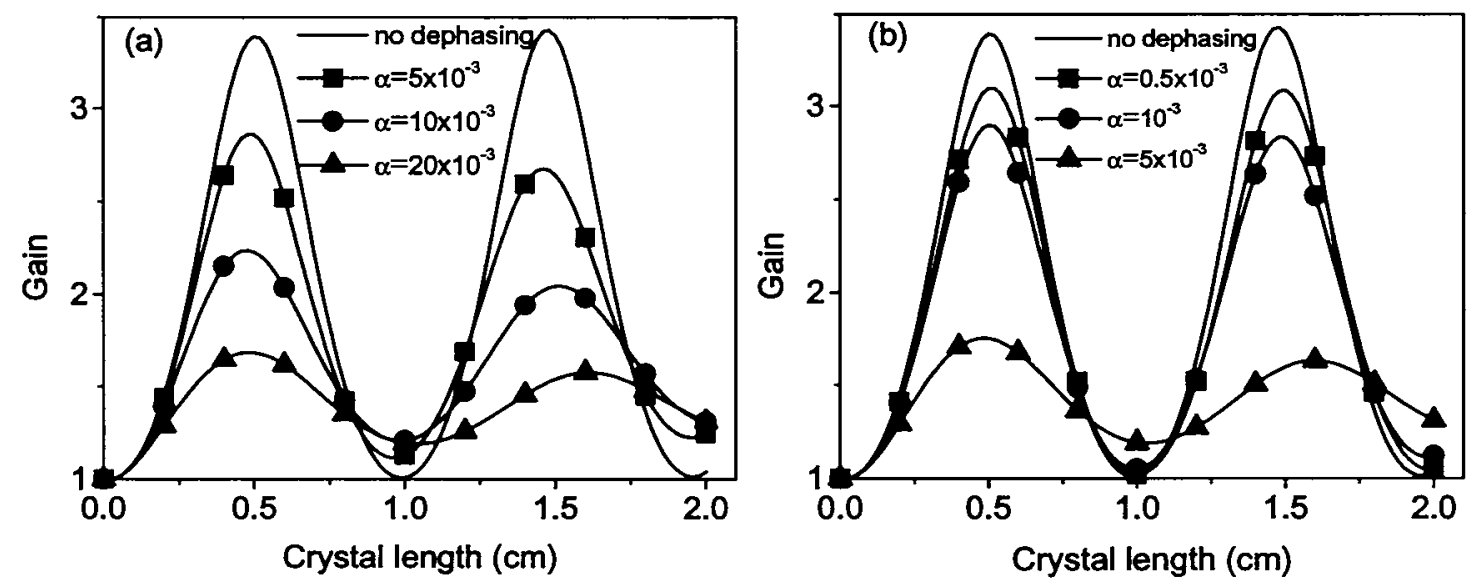

Figure 8.6. Large signal gain in BBO for different divergence parameters, for (a) gaussian beams, (b) top-hat beams. Simulation parameters: $\lambda_{s}=1054 \mathrm{~nm}, \lambda_{p}=532$ $\mathrm{nm}, I_{p}=500 \mathrm{MW} / \mathrm{cm}^{2}, 50 \mathrm{~mJ}$ pump, $10 \mathrm{~mJ}$ seed.,
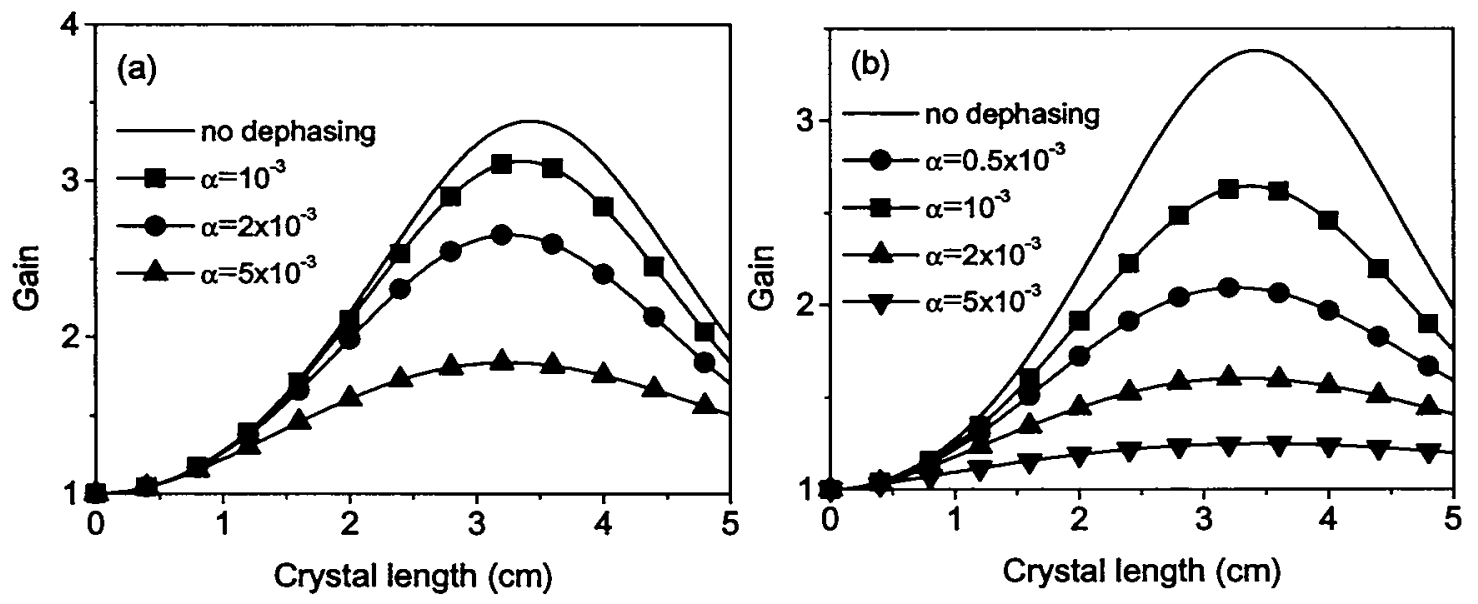

Figure 8.7. Large signal gain in KDP for different divergence parameters, for (a) gaussian beams, (b) top-hat beams. Simulation parameters: $\lambda_{s}=1054 \mathrm{~nm}, \lambda_{p}=532$ $\mathrm{nm}, I_{p}=500 \mathrm{MW} / \mathrm{cm}^{2}, 50 \mathrm{~mJ}$ pump, $10 \mathrm{~mJ}$ seed.

To evaluate maximum conversion efficiency in OPA, large signal gain configurations need to be analyzed. Next, gain is calculated when Power $_{\text {pump }}=5$ Power $_{\text {seed }}$. This leads to strong depletion of the pump wave and back conversion. Results for BBO, KDP and LBO 
are shown in Figs. 8.6-8.8, for gaussian and top-hat beam profiles. An important difference exists between our results and the results of simple calculations in which a single plane wave with a single averaged value of phase mismatch is considered. A plane wave analysis without angular distribution results in cycles of conversion and back conversion that occur with specific frequency and consistently attain the same maxima and minima. The results presented here show conversion behavior similar to the behavior of a damped oscillator, where successive oscillations become less pronounced. This is the result of a large number of plane waves interacting with different wave vector mismatch and subsequent different conversion and back conversion rates for plane wave components across the spatial frequency spectrum. The damped oscillatory conversion behavior is consistent with the previous calculation ${ }^{106}$ for second-harmonic generation.
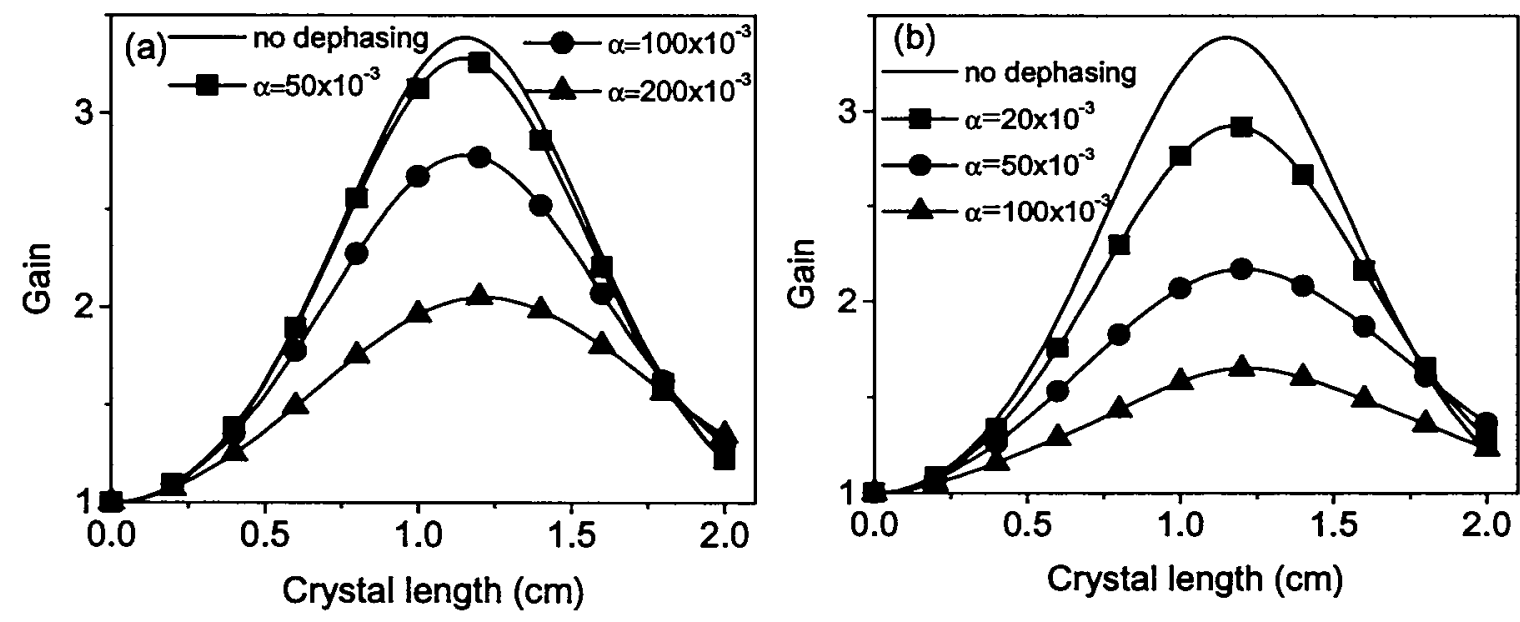

Figure 8.8. Large signal gain in noncritically phase-matched LBO for different divergence parameters, for (a) gaussian beams, (b) top-hat beams. Simulation parameters: $\lambda_{s}=1054 \mathrm{~nm}, \lambda_{p}=532 \mathrm{~nm}, I_{p}=500 \mathrm{MW} / \mathrm{cm}^{2}$.

Top-hat beams are preferred in optical parametric processes due to uniform conversion. However, for the same radial intensity variance, gaussian beams are less 
sensitive to angular divergence than top-hat beams in optical parametric amplification. If the spatial frequency spectra of gaussian and top-hat beams are compared, it is found that the gaussian's spectrum has greater energy content in its central lobe than the top-hat's. This can be shown by considering a spatial frequency window defined by the first zero of the Bessel function for the spatial frequency spectrum of the top-hat beam. While the tophat beam has only $84 \%$, the gaussian beam with the same radial intensity variance has $99.9 \%$ of its energy concentrated in the same enclosed area. It is this difference that is responsible for the observed variation in the angular sensitivity. Common nonlinear crystals in critically phase-matched configurations typically exhibit narrow angular tolerances. Frequently, only the central lobe of the spatial frequency spectrum of a top-hat beam is included in the angular bandwidth for optical parametric amplification. Since the rate of difference-frequency generation has a strong nonlinear dependence on beam intensity, a significant drop in gain is expected with reduced effective beam intensity. In an alternative view, the high spatial frequencies of the pump experience rapid cycles of conversion and back conversion, not contributing significantly to the overall conversion.

\subsection{High conversion criteria}

Eimerl ${ }^{108}$ has previously studied second-harmonic generation and concluded that the conversion efficiency in second-harmonic generation is determined exclusively by the beam peak power and beam quality in critically phase-matched crystals. While increasing the beam intensity by telescoping the beam decreases the required crystal length, there is 
no impact on the overall conversion efficiency. This result can be extended to optical parametric amplification. For this case, the simplified expression for small signal gain is

$$
G=\frac{1}{4} \exp \left(2 g_{0} L\right) \operatorname{sinc}^{2}\left(\frac{\Delta k L}{2}\right)
$$

where $g_{0}=K \sqrt{I_{p}}$ is the gain coefficient, $I_{p}$ is the intensity of the pump, $L$ is the crystal length, and $K=4 \pi d_{e f f} / \sqrt{2 \varepsilon_{0} n_{p} n_{s} n_{i} c \lambda_{s} \lambda_{p}}$. In critical phase matching, the wave vector mismatch $\Delta k$ can be approximated by the linear function of divergence angle for small angle:

$$
\Delta k L=\beta_{\theta} \Delta \theta L=\frac{\beta_{\theta}}{4} \frac{M^{2} \lambda}{\pi w} L
$$

where $\beta_{\theta}=d(\Delta k) / d \theta$ is the angular sensitivity for a critically phase-matched nonlinear crystal. We can now rewrite the expression for small signal gain for radially symmetric beams as

$$
G=\frac{1}{4} \exp \left(\frac{2 K}{\sqrt{\pi}} \sqrt{P_{p}} \frac{L}{w}\right) \operatorname{sinc}^{2}\left(\frac{\beta_{\theta}}{8} \frac{M^{2} \lambda}{\pi} \frac{L}{w}\right),
$$

where $P_{p}$ is the peak power of the pump beam. While telescoping the beam changes beam radius $w$, the ratio $L / w$ remains invariant for the same gain. The problem of obtaining maximum gain reduces to selecting the proper ratio of crystal length $L$ to beam radius $w$ for a particular pump peak power.

Dephasing can be reduced in uniaxial crystals by using elliptical beams, elongated in the principal plane of the crystal. Elliptical beams take advantage of the anisotropy in angular sensitivity, reducing dephasing while enabling the same pump intensity. Compared to a radially symmetric beam of the same intensity, dephasing is reduced by a 
factor of $\sqrt{A}$, where $A$ is the beam aspect ratio. As a consequence, the beam quality parameter $M^{2}$ can be $\sqrt{A}$ times greater compared to a radially symmetric beam. The limit of usability of this scheme in uniaxial crystals is determined by dephasing due to divergence in the insensitive direction. This dephasing in the insensitive direction starts to compete with dephasing due to divergence in the sensitive direction at large aspect ratios. This aspect ratio in $\mathrm{BBO}$ is calculated to be $\sim 20$. Another advantage of using elliptical beams in uniaxial crystals is the reduction of the impact of walk-off. Walk-off occurs for the extraordinary polarized beam and is directed in the crystal sensitive direction. Using elliptical beams increases the overlap of the ordinary and extraordinary beams. This results in better conversion efficiency, particularly when intersecting beams are small and interaction lengths are long.

In noncritical phase matching, phase mismatch can be approximated by a quadratic dependence on the divergence angle

$$
\Delta k L=\gamma_{\theta} \Delta \theta^{2} L=\frac{\gamma_{\theta}}{16} \frac{M^{4} \lambda^{2}}{\pi^{2} w} \frac{L}{w},
$$

where $\gamma_{\theta}=d^{2}(\Delta k) / d \theta^{2}$ is the angular sensitivity for noncritical phase matching. Increasing the beam size can increase the conversion efficiency in noncritically phasematched crystals. Contrary to intuition, weaker beam focusing enables higher conversion in noncritically phase-matched crystals. The obvious limit of practicality of this result is the availability of large crystals of sufficient quality. Otherwise, the limit is set by the dephasing sources that are directly proportional to crystal length, such as spectral and temperature bandwidths. 
In a simplified analysis, we now derive the criterion for beam quality that allows high conversion efficiency in OPAs. By Eq. (8.16), with dephasing neglected, the desired small signal gain can be obtained for a given value of pump peak power by selecting the appropriate ratio of crystal length and beam radius

$$
L / w=\frac{\sqrt{\pi}}{2 K \sqrt{P}} \ln (4 G) .
$$

The dephasing term for the selected ratio $L / w$ is

$$
\begin{gathered}
\operatorname{sinc}^{2}\left(\frac{\Delta k L}{2}\right)=\operatorname{sinc}^{2}\left(\frac{\beta_{\theta}}{16 \sqrt{\pi}} \frac{M^{2} \lambda \ln (4 G)}{K \sqrt{P}}\right), \\
\operatorname{sinc}^{2}\left(\frac{\Delta k L}{2}\right)=\operatorname{sinc}^{2}\left(\frac{\gamma_{\theta}}{32 \pi^{3 / 2}} \frac{M^{4} \lambda^{2} \ln (4 G)}{K w \sqrt{P}}\right),
\end{gathered}
$$

for critically and noncritically phase-matched crystals, respectively. If the requirement for high conversion efficiency is defined as $\operatorname{sinc}^{2}(\Delta k L / 2)>0.5$, or $\Delta k L / 2<1.39$, a criterion is obtained for required beam quality for lasers pumping OPAs:

$$
\begin{gathered}
M^{2}<1.39 \times 16 \sqrt{\pi} \frac{K \sqrt{P}}{\beta_{\theta} \lambda \ln (4 G)}, \\
M^{4}<1.39 \times 32 \pi^{3 / 2} \frac{K w \sqrt{P}}{\gamma_{\theta} \lambda^{2} \ln (4 G)},
\end{gathered}
$$

for critically and noncritically phase-matched crystals, respectively. Note that for critical phase-matched operation the required beam quality $M^{2}$ does not depend on the beam radius. For noncritical phase matching, the required $M^{2}$ scales as the square root of the beam radius.

An important consideration in OPA design concerns a simple difference between harmonic generation and seeded OPAs. While second-harmonic generators build up 
without an incident harmonic beam, seeded OPAs have radically different boundary conditions, where seed beam intensity is typically many orders of magnitude greater than the zero-point field fluctuation. Seed power influences the overall conversion efficiency in the OPA. A simple calculation was performed to determine maximum conversion in an OPA as a function of seed power. The results are shown in Fig. 8.9(a), without walk-off, and in Fig. 8.9(b), with walk-off.
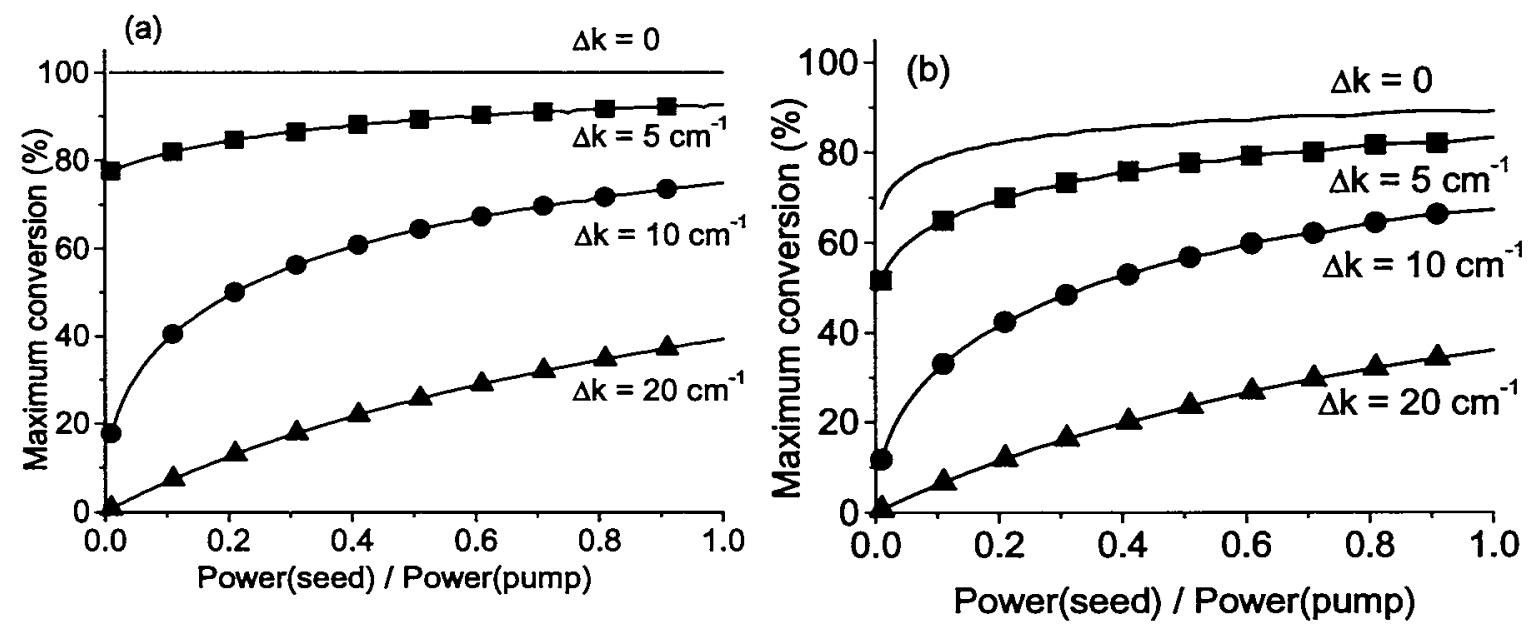

Figure 8.9. Maximum conversion in $\mathrm{BBO}$ as a function of the ratio of seed and pump input, for (a) no walk-off included, (b) walk-off included. Simulation parameters: $\lambda_{s}=1054 \mathrm{~nm}, \lambda_{p}=532 \mathrm{~nm}, I_{p}=1 \mathrm{GW} / \mathrm{cm}^{2}$

The maximum conversion of the pump beam to signal and idler is plotted as a function of the ratio of seed and pump peak power, for different values of wave vector mismatch $\Delta k$. A single plane wave with wave vector mismatch $\Delta k$ is assumed. The evaluated mixing process is $1054 \mathrm{~nm}(\mathrm{o})+1074 \mathrm{~nm}(\mathrm{o})=532 \mathrm{~nm}(\mathrm{e})$, with pump waist size $2 \mathrm{~mm}$ (top-hat), and pump intensity of $1 \mathrm{GW} / \mathrm{cm}^{2}$. Maximum conversion is found by varying the crystal length with constant input intensity. With $\Delta k=0$ and in the absence of 
walk-off, maximum conversion is $100 \%$ and it is independent of the seed power. As the wave vector mismatch increases, higher conversion efficiency is possible when the input seed intensity increases. With the inclusion of walk-off, even zero wave vector mismatch leads to a maximum conversion of $\sim 85 \%$ when Power $_{\text {seed }} /$ Power $_{\text {pump }}=1$. The efficiency can be improved by increasing the ratio Power $_{\text {seed }} /$ Power $_{\text {pump }}$, asymptotically approaching $100 \%$ when Power $_{\text {seed }} /$ Power $_{\text {pump }} \rightarrow \infty$.

\subsection{Conclusion}

This Chapter explored the angular effects in optical parametric amplification using a numerical model of difference-frequency generation. A numerical model was developed, based on plane wave decomposition. The model can be used to isolate and evaluate the influence of spatial frequency content of real laser beams on the performance of devices based on optical parametric amplification. It was found that top-hat beams exhibit greater sensitivity to angular dephasing than gaussian beams with the same radial intensity variance. This result is attributed to the more broadly distributed angular spectrum of the top-hat beam.

Criteria were derived for required beam quality in critically and noncritically phasematched optical parametric amplification. It was shown that the required beam quality is invariant with respect to the beam diameter in critical phase matching. In noncritical phase matching, the beam quality requirements are more relaxed as the beam radius increases. Practical tolerances were determined on beam quality for gaussian and top-hat beams in several commonly used nonlinear crystals. 
The conversion efficiency sensitivities with respect to the beam ellipticity and seed power was also studied. Beam quality requirements can be relaxed by using elliptical beams in critically phase-matched crystals by taking advantage of the large anisotropy in the angular sensitivity. The maximum conversion in an OPA is increased for greater incident seed power, for all values of wave vector mismatch.

At an arbitrary point, the angular decomposition model for optical parametric amplification describes the input beams more realistically than single plane wave models. A complete description would include a variable intensity profile in the spatial and temporal domains. Such an inclusion would necessitate considerably more computational power than this simple model. As a stand-alone calculation, the angular decomposition model nevertheless provides insight into angular effects in OPAs and in estimating their relative magnitude. 


\section{Chapter 9}

\section{Applications of optical parametric}

\section{chirped pulse amplification}

The motivation for development and use of OPCPA stems from applications that benefit from the unique characteristics and scalability of the technology. Here, several potential important applications are briefly presented. The use of OPCPA in the front end of a fast ignitor laser for inertial confinement fusion is discussed in section 9.1. This is followed by a brief description of a possible multi-PW source for high-field science based entirely on OPCPA in section 9.2. Materials processing applications are addressed in section 9.3, along with previous discussions in Chapter 7. Finally, more speculative possibilities for use of OPA and OPCPA for amplification of wavelengths used in telecommunications are given in section 9.4.

\subsection{Fast ignitor for inertial confinement fusion}

The origin of interest in laser-driven inertial confinement fusion (ICF) ${ }^{109}$ comes from the first realization that lasers enable rapid, high-energy deposition in a very small volume. Direct-drive ${ }^{110}$ and indirect-drive ${ }^{111}$ schemes for ICF were developed and tested 
in large experimental facilities such as $\mathrm{Nova}^{112}$ and Omega. ${ }^{113}$ Laser-driven ICF is anticipated to lead to the first achievement of ignition with the development of the National Ignition Facility (NIF). ${ }^{114}$

Some of the problems facing the conventional laser-driven ICF are the very stringent requirements on the laser driver pulse energy and uniformity, and too low anticipated wall-plug efficiency for future fusion energy production. In a more recently proposed approach, a short-pulse laser can be used to produce much higher fusion gain and simultaneously relax the requirements imposed on the laser driver. Termed fast ignition, ${ }^{115,116}$ the process starts with isobaric spherical compression, which leads to a lower density and a substantially lower temperature than the adiabatic compression in conventional ICF. At the point of maximum compression, the ponderomotive force associated with a short energetic laser pulse is used to create a thin channel in the compressed plasma. Short laser pulse transfers some of its energy to suprathermal electrons, which exchange their energy with thermal electrons and ions through rapid collisions in the central dense part of the compressed pellet. The result is the heating of the pellet center to $5-10 \mathrm{keV}$, which initiates the fusion reaction. The fusion burn propagates rapidly through the remaining fuel, with much higher burnup efficiency. The expected gain from a fast ignitor shot is one order of magnitude higher than from the conventional ICF shot. The first subscale demonstration of fast ignition has been performed, and an order of magnitude greater number of fusion neutrons has been detected compared to reaction that uses conventional laser drive at a same energy, but without the short-pulse fast ignitor laser. ${ }^{117}$ 
The requirements on the short-pulse ignitor laser are formidable: a pulse energy of $10-100 \mathrm{~kJ}$ has to be delivered in a time shorter than the pellet disassembly time (1-20 ps). ${ }^{118}$ The challenge of obtaining short pulses from an amplification process that occurs over 13-14 orders of magnitude was previously met by a hybrid laser system that included a broad-bandwidth front end design. ${ }^{44}$ The front end relied on Ti:sapphire regenerative amplifiers to produce the majority of gain. ${ }^{119}$ It is proposed that the front end of the fast ignitor laser can be simplified by replacing Ti:sapphire regenerative amplifiers with a broad-bandwidth OPCPA.

One possible conceptual design of the fast ignitor laser on NIF is presented in Fig. 9.1. The system starts with a short-pulse Ti:sapphire or Nd:glass oscillator, which produces 1 ps pulses at $1054 \mathrm{~nm}$. The pulses are stretched to $1 \mathrm{~ns}$ and amplified in OPA pumped by a pump pulse from the NIF preamplifier module (PAM). ${ }^{120}$ The PAM produces $1 \mathrm{~ns}, 1054 \mathrm{~nm}$ pulses that are top-hat shaped temporally to facilitate highefficiency doubling. The pulses are frequency-doubled to $532 \mathrm{~nm}$, and maximum pulse energy of $2 \mathrm{~J}$ is obtained.

\begin{tabular}{|c|c|c|c|c|}
\hline $\begin{array}{c}\text { Crystal } \\
\text { Dimensions }\end{array}$ & $\begin{array}{c}\text { Input seed } \\
\text { Energy/diameter }\end{array}$ & $\begin{array}{c}\text { Input pump } \\
\text { Energy/diameter }\end{array}$ & $\begin{array}{c}\text { Pump } \\
\text { intensity }\end{array}$ & $\begin{array}{c}\text { Output signal } \\
\text { energy }\end{array}$ \\
\hline BBO & $1 \mathrm{~nJ}$ & $300 \mathrm{~mJ}$ & 840 & $280 \mu \mathrm{J}$ \\
$8 \times 8 \times 15 \mathrm{~mm}^{3}$ & $1 \mathrm{~mm}$ & $6.7 \mathrm{~mm}$ & $\mathrm{MW} / \mathrm{cm}^{2}$ & \\
\hline $\mathrm{BBO}$ & $280 \mu \mathrm{J}$ & $1.7 \mathrm{~J}$ & $\begin{array}{c}770 \\
\mathrm{MW} / \mathrm{cm}^{2}\end{array}$ & $650 \mathrm{~mJ}$ \\
$18 \times 18 \times 11 \mathrm{~mm}^{3}$ & $16 \mathrm{~mm}$ & $16.7 \mathrm{~mm}$ & \\
\hline
\end{tabular}

Table 9.1. OPA design parameters for the OPCPA-based front end of the fast ignitor laser

The seed pulse is injected into a two-stage OPA based on $\beta$-barium borate (BBO). A fraction of the PAM pulse energy $(300 \mathrm{~mJ})$ is split from the pump pulse and used to pump the first BBO OPA stage, while the remaining energy (1.7 J) is used to pump the 
second BBO OPA stage. The important anticipated system design parameters are summarized in Table 9.1. The intensity is chosen such that the damage threshold in BBO is avoided with 1 ns pulses. A top-hat transverse profile is chosen for pump and seed.

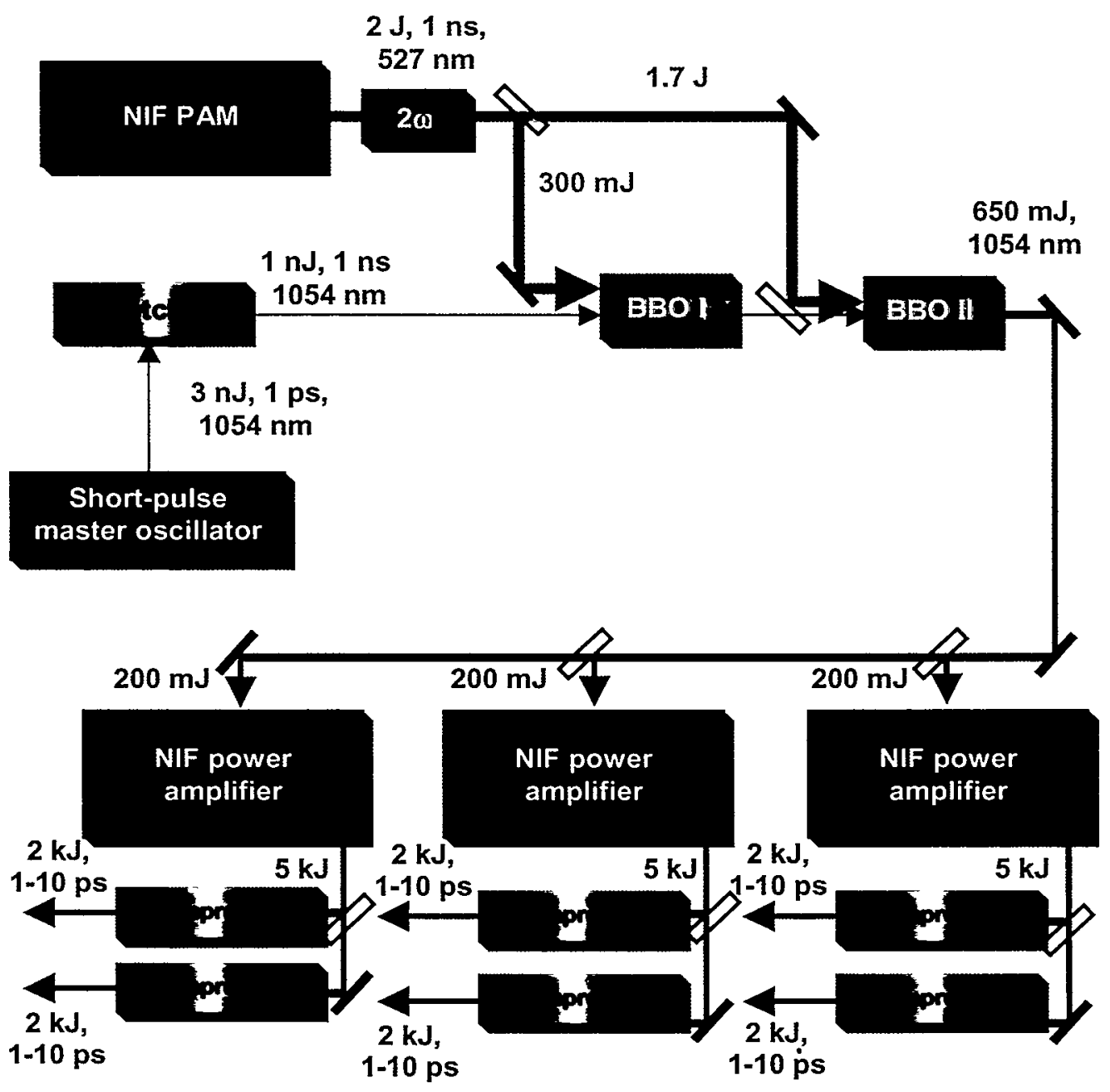

Figure 9.1. A possible conceptual design for the NIF fast ignitor laser based on an OPCPA front end

Signal output pulse energies from the first and second crystal are $280 \mu \mathrm{J}$ and 650 $\mathrm{mJ}$, respectively. The anticipated temporal shape of the stretched seed and amplified 
signal in OPA is shown in Fig. 9.2. The pulse amplified in the OPCPA front end is split into three $\sim 200 \mathrm{~mJ}$ pulses, and they are used to seed three NIF power amplifiers.

The amplified pulse energy after four passes through a NIF amplifier is $5 \mathrm{~kJ}$. The amplified pulse is subsequently split into two $2.5 \mathrm{~kJ}$ pulses after each amplifier. Two compressors are used for compression of pulses amplified by each amplifier to avoid the grating damage. The total recompressed pulse energy from 6 compressors is $12 \mathrm{~kJ}$. Separation of the compressor gratings can be adjusted to produce pulses continuously tunable between 1 and $10 \mathrm{ps.}$

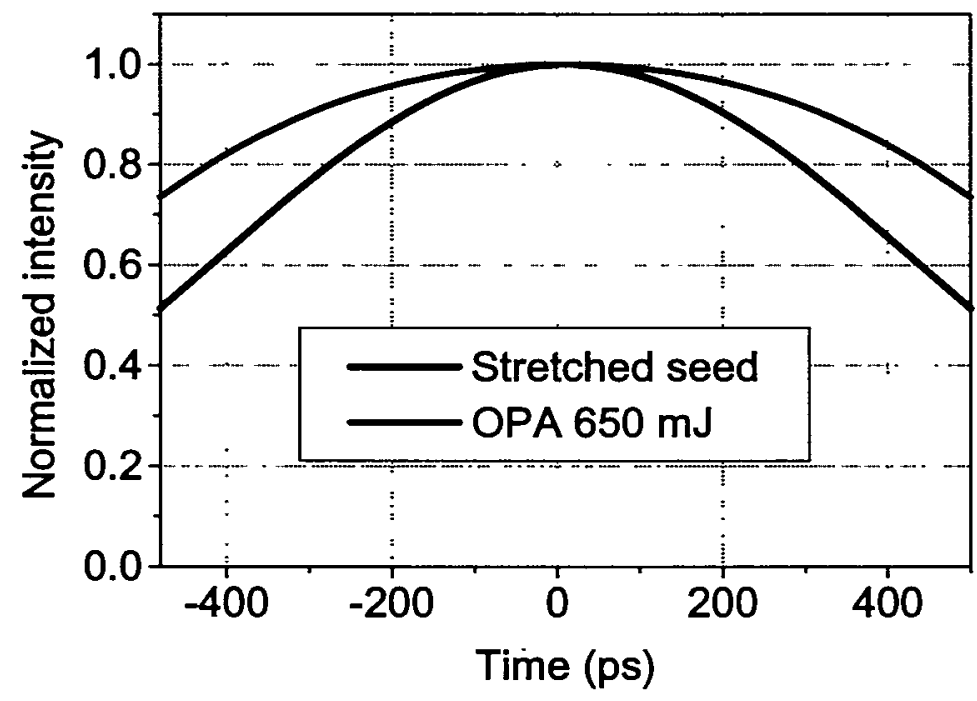

Figure 9.2. Temporal (spectral) shape of the signal after stretcher, first $\mathrm{BBO}$ amplifier and second $\mathrm{BBO}$ amplifier

Recompression is performed using high-efficiency, high damage threshold dielectric diffraction gratings ${ }^{121}$ to obtain $2 \mathrm{~kJ}, 1-10$ ps pulses. For this application, it is necessary to scale the current dielectric diffraction gratings to large apertures previously obtained only with metallic gratings. In addition, it will be necessary to convert 5 NIF beamlines into short-pulse lasers to obtain target pulse energies in the desired range of 10 
$\mathrm{kJ}$. The fast ignitor laser on NIF based on an OPCPA front end can benefit from the simplicity and robustness of the single-pass broadband high-gain stage.

\subsection{High-field basic science}

One of the most exciting results of the development of short-pulse lasers is the opening of the new high-intensity regime for basic science experiments. As an example of the conditions that occur in the focused laser spot, it is illustrative to consider the focused intensity of $10^{21} \mathrm{~W} / \mathrm{cm}^{2}$, accessible with the JanUSP laser. ${ }^{9}$ The electric field at the focal spot exceeds $3 \times 10^{11} \mathrm{~V} / \mathrm{cm}$, which is almost 5 orders of magnitude greater than the acceleration gradient in modern particle accelerators. Violent acceleration of particles in the focal spot resembles the conditions occurring in vicinity of black holes. The light pressure at the focal spot exceeds $300 \mathrm{Gbar}$, which is comparable to pressures that occur in extreme astrophysical objects. Finally, the magnetic field at the focus approaches $10^{5}$ $\mathrm{T}$, resulting in anharmonic relativistic motion of particles in the laser focus and intense, low-divergence $\mathrm{x}$-ray emission.

High-field phenomena that can be investigated using ultraintense short-pulse lasers include nuclear fusion from atomic clusters, ${ }^{122}$ photon-photon scattering in vacuum, ${ }^{123}$ nonlinear Thomson and Compton scattering, ${ }^{124}$ laser-plasma particle acceleration, ${ }^{125}$ laser-induced nuclear reactions, ${ }^{126}$ relativistic plasma physics, ${ }^{127}$ and high-energy astrophysical processes, such as supernovae and gamma-ray bursts. ${ }^{128}$

With the short-pulse energy limited by the size and damage threshold of compressor gratings, two main approaches have been proposed to scale the peak power 
beyond $10^{15} \mathrm{~W}$. The first proposal suggests laser pulse compression in plasma through relativistic self-focusing ${ }^{129}$ or stimulated Raman backscattering, ${ }^{130}$ with the maximum power scaling to $>10^{20} \mathrm{~W}$. However, pulse compression in plasma is relatively unexplored. One of the significant problems with those proposals is the required plasma uniformity for uniform recompression, which is a difficult requirement to satisfy over relatively large aperture and length. ${ }^{131}$

Current large glass laser systems are fundamentally limited by the relatively narrow gain bandwidth in Nd:glass, which does not allow amplification of pulses shorter than several hundreds of $\mathrm{fs}$ without regenerative pulse shaping. ${ }^{132}$ Another possibility exists to scale the power to $>10^{16} \mathrm{~W}$ using the existing compression grating technology and a large OPA amplifier in a CPA system pumped by a large Nd:glass laser. ${ }^{13,133}$

Recent rapid-growth large aperture scaling of nonlinear KDP crystals ${ }^{75}$ allows construction of a high-energy all-OPCPA amplification system which could produce peak powers in excess of $10^{16} \mathrm{~W}$ in $20 \mathrm{fs}$ using large-aperture metallic gratings. ${ }^{43}$ High beam quality that can be obtained from OPCPA would enable intensities as high as $10^{23}$ W/cm² ${ }^{2}$ Figs. 9.3 and 9.4 show two possible conceptual designs for such OPCPA system based on KDP.

In the degenerate system (Fig. 9.3 (a)), second harmonic of the glass laser (527 $\mathrm{mm}$ ) is used to pump the KDP OPA operating at an arbitrary wavelength near $1054 \mathrm{~nm}$. While broad bandwidth amplification in nearly degenerate KDP OPCPA has been demonstrated before, ${ }^{65}$ availability of short-pulse $(<20 \mathrm{fs})$ sources at degenerate wavelength near $1 \mu \mathrm{m}$ represents the greatest problem with this approach. It is conceivable that such source could be built in the future using spectral broadening by 
self-phase modulation in a bulk material or a photonic fiber. Pulses would be stretched to $\sim \mathrm{ns}$, and amplified to $\sim 500 \mathrm{~J}$ without bandwidth limitation in the KDP OPA. Using a metallic grating compressor with overall efficiency of $60 \%$, such system could produce $15 \mathrm{PW}$ of peak power power.

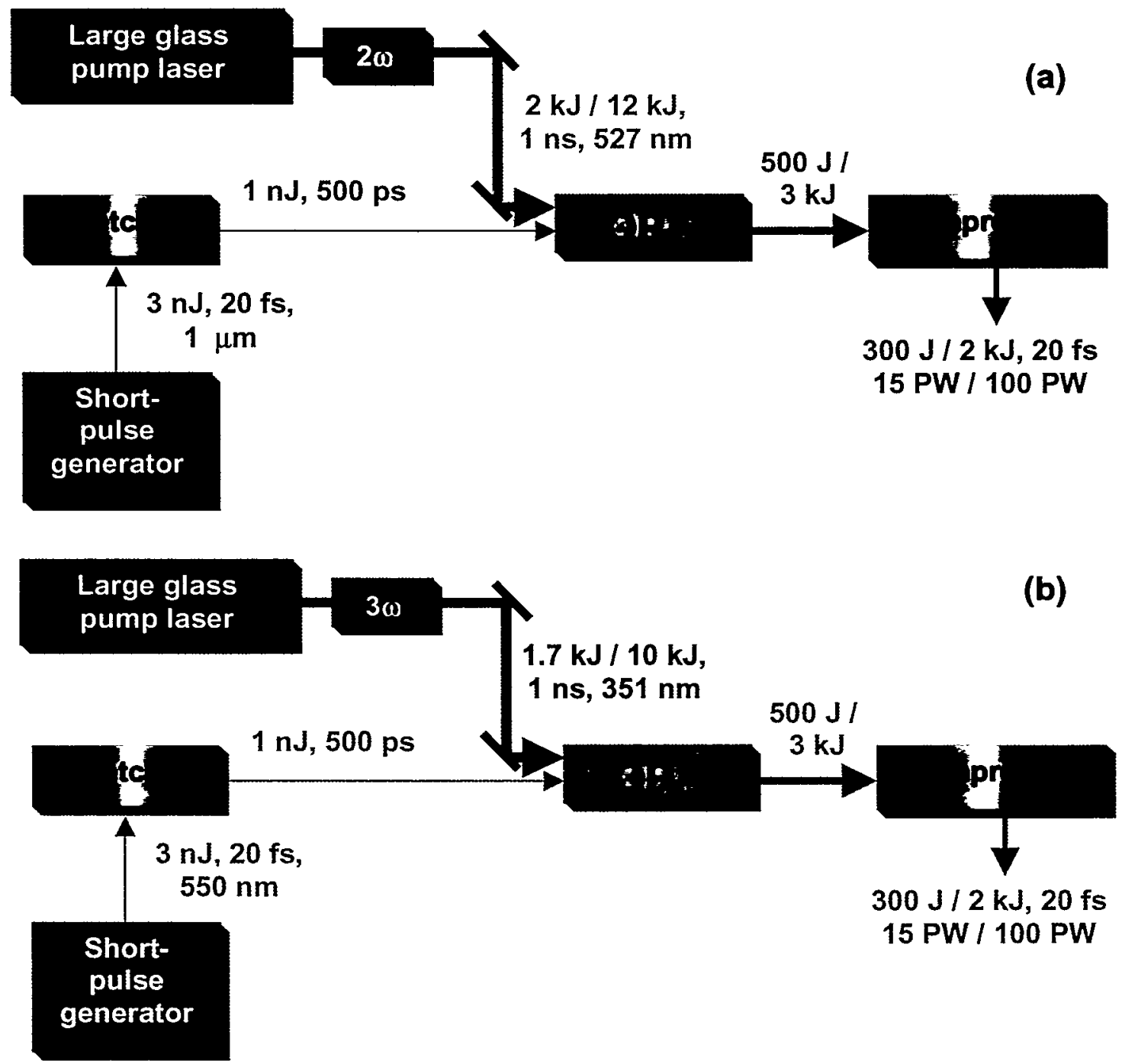

Figure 9.3. Conceptual design for a 15 PW / 100 PW all-OPCPA-based source, operating (a) near degeneracy, and (b) away from degeneracy. Compressor based on metallic gratings may enable peak power of $15 \mathrm{PW}$, while a compressor based on dielectric gratings could be scaled to $100 \mathrm{PW}$. 
Another possibility would be the use of KDP in a nondegenerate system (Fig. 9.3 (b)), pumped by the third harmonic of a large Nd:glass laser (351 nm). Like in the system shown in Fig. 9.3 (a), generation of ultrashort seed pulses remains to be addressed. Such broadband nondegenerate KDP OPA would require seed pulses centered near $550 \mathrm{~nm}$. As a potential benefit of amplification at shorter wavelengths, the maximum focused intensity would be increased. Additionally, energy loss by conversion of the fundamental frequency of the pump laser to its third harmonic would be compensated by the favorable 2:1 energy splitting between signal and idler beams.

A possibility of nondegenerate broadband amplification in KDP at the wavelength more readily obtainable from standard Ti:sapphire oscillators deserves further study. As shown in section 5.3, angular dispersion and temporal modulation can be used to obtain broadband, uniform amplification over a wide range of center wavelengths. Using tailored angular dispersion in combination with a noncollinear angle for the desired center wavelength may enable the use of common ultrashort pulse Ti:sapphire oscillators for nondegenerate amplification in large-aperture KDP. Such approach would require a special dispersion-producing element, whose output would have to be relay imaged in a multi-crystal OPCPA system.

Advances in aperture and damage threshold of compressor gratings will enable even greater peak power using OPCPA. Assuming the development of large size dielectric diffraction gratings, scaling to $2 \mathrm{~kJ} / 20 \mathrm{fs}$ may be possible, increasing the peak power to $100 \mathrm{PW}$ or $0.1 \mathrm{EW}$ (exawatt). Focused intensities on target in that case may approach $10^{24} \mathrm{~W} / \mathrm{cm}^{2}$, allowing investigations of high-field physics in a new regime. 


\subsection{Materials processing}

While materials processing has been one of the traditional industrial applications of lasers, the use of short pulses for materials processing has received much attention only recently. ${ }^{134}$ The advantage of using short laser pulses for materials processing is in the fundamentally different mechanism of material removal by short pulses when compared to long pulses. ${ }^{135,136}$ In the long pulse regime ( $\left.>10 \mathrm{ps}\right)$, material removal is dominated by heat deposition and melting. As the pulse width is reduced, the characteristic time for thermal diffusion exceeds the laser pulse duration. When the intensity of the short laser pulse incident on a dielectric exceeds the multiphoton ionization threshold, the material is ablated from the surface without thermal deposition. The ablation occurs in the form of plasma plume.

The lack of thermal deposition and the absence of melting allow very highprecision machining of dielectrics with short-pulse lasers. As an additional advantage, laser cutting can be applied in situations when thermal deposition in the material is prohibitive, such as in cutting of high explosives. ${ }^{137}$

Once the critical pulse energy is exceeded, the cutting rate in short-pulse machining is dependent primarily on the laser repetition rate. Hence increasing the cutting rate requires development of high repetition rate, high average power short-pulse lasers. With a limited number of materials suitable for short-pulse amplification, high average power scaling becomes a difficult requirement to satisfy. Recent advances in high average power short-pulse laser technology include the use of reduced quantum defect materials, such as Yb:YAG, ${ }^{138}$ and the use of thermal eigenmode amplification in Ti:sapphire. ${ }^{139}$ 
With its broad bandwidth and zero intrinsic heat load, OPCPA is a serious candidate for the next generation of high average power short-pulse lasers for materials processing. Chapter 7 describes the energy limits and possible approaches to pump lasers which may permit the future power scaling. Conditions exist to scale OPCPA to kilowattlevel average power in nonlinear crystals with favorable thermal properties, such as BBO. This development would allow unprecedented rates of material removal using short pulses. Most importantly, the development of this technology will depend on the advances in high average power nanosecond pump lasers, particularly reducing their pulse width, improving beam quality and allowing spatial and temporal shaping.

\subsection{Broadband amplification for telecommunications}

In the growing field of optical telecommunications, broadband amplification is becoming a necessity. The transmission rate of optical information through optical fibers has been steadily increasing through increases in bandwidth. Today, typical broadband amplifiers used in telecommunications include Raman amplifiers ${ }^{140}$ and Er:fiber - based laser amplifiers. ${ }^{141}$ A typical requirement for those amplifiers is high-fidelity, low-noise amplification with a broad bandwidth $(\sim 200 \mathrm{~nm})$ around the central frequency of 1550 nm.

As outlined in section 3.7, OPA is a low-noise amplification process, which has a useful characteristic of transferring the pump aberrations to the idler wave. This OPA characteristic, coupled with the broad bandwidth and tunability, represents a potential advantage of OPA for amplification of signals used in telecommunications. For this 
development to occur, suitable nonlinear materials with high second-order nonlinearities and broad acceptance angles will have to be identified. Such OPAs will have to be pumped by pump lasers with relatively low peak power, such as laser diodes, or by small fiber lasers. The likely candidates for materials for OPAs in telecommunications include engineerable periodically-poled materials and organic materials that exhibit very large nonlinear coefficients. 


\section{Chapter 10}

\section{Conclusion}

Optical parametric chirped pulse amplification is a promising technology that has a potential to deliver unprecedented levels of peak and average power. While the experimental work described in this dissertation is far from representing the ultimate limits of the technology, it includes several achievements and proposals that represent advances in the state of the art. In the concluding text, the most significant conclusions are reiterated, along with proposals for future improvements.

A numerical model was developed and successfully applied to the case of small signal and pump depletion regime in OPCPA. Advantage was taken of the negligible impact of group velocity dispersion in nanosecond OPCPA and the numerical model was simplified to a 3-dimensional, from the usual 4-dimensional case. The electric field at any point of the crystal is evaluated starting from arbitrary spatio-temporal modes for signal, idler and pump, with noncollinearity and Poynting vector walk-off included. Systematic transverse misalignments of the signal and pump were used as an adjustable parameter in the model. Future improvements in the code should include the explicit account of diffraction, allowing treatment of beams with short confocal parameters.

OPCPA was demonstrated as a replacement for Ti:sapphire regenerative amplifier in a CPA system. A novel double-crystal design was demonstrated for the high-gain preamplifier. Pumped by a commercial pump laser, this OPCPA system resulted in a 50- 
fold increase in energy and a 10 -fold improvement in conversion efficiency compared to similar previous experiments by other groups. Moreover, recompression to $310 \mathrm{fs}$ is the best recompression of any $1 \mu \mathrm{m}$ OPCPA so far pumped by a commercial pump laser. In the future, a reduction of recompressed pulse width in $1 \mu \mathrm{m}$ OPCPA may be obtained by improvements in the stretcher design. In any case, OPCPA proved to be a viable alternative to $1 \mu \mathrm{m}$ Ti:sapphire regenerative amplifier for the front end of high-power glass short-pulse lasers.

In the nondegenerate OPCPA experiment, the ultrashort capability of OPCPA was demonstrated at a level of $60 \mathrm{fs}$. This is an improvement of almost one order of magnitude compared to previous OPCPA work, and represents the first recompression of pulses amplified in highly nondegenerate OPCPA. Achievement of good conversion efficiency in the spatio-temporal window defined by the seed pulse, and the demonstration of ultrashort pulse recompression provides an argument for scalability of the technology to ultrahigh peak power. Future directions include development of appropriate short-pulse sources suitable for ultrashort pulse amplification in KDP and experimental verification of the concept of broadband amplification at an arbitrary center wavelength using angular dispersion in combination with noncollinear angle.

The first hybrid chirped pulse amplification system described in Chapter 5 goes beyond the efficiency possible in OPCPA alone, when pumped by a tabletop commercial pump laser. In a simple setup that uses only a fraction of the energy from a commercial pump laser, energies of up to $45 \mathrm{~mJ}$ were obtained. Simple improvement should lead to $40-50 \%$ overall conversion efficiencies. In addition to performance benefits, the system is cheap, robust and safer than then one that uses regenerative amplification because of the 
absence of high-voltage electro-optic modulators. It is believed that this approach will find wide application in the future, particularly because of the possibility of generating 10-TW pulses in an all-passive setup using a single, commercial tabletop Q-switched pump laser.

A numerical model for high average power OPA was developed and described in Chapter 7. The model addresses absorption-induced heat load in OPA and calculates the resulting conversion in a self-consistent way. The results of calculations indicate that average power of several hundred watts can be obtained from a single OPCPA crystal without complicated cooling or pre-stressing. Additionally, beam quality can be preserved at an expense of conversion efficiency by simple crystal angle adjustments. An approach is presented that can increase the average power in OPA by cascaded difference-frequency generation. Preliminary designs and results on the future pump laser technology are also presented. While high average power short-pulse generation by OPCPA has many promising characteristics, future work will depend on development of suitable pump sources.

An important question in the design of OPCPA is the required beam quality of the pump lasers. This problem was approached using a plane wave decomposition model described in Chapter 8. Several popular nonlinear crystals were evaluated, and conclusions were made on the maximum allowed angular deviation and corresponding beam quality. Finally, high conversion efficiency criteria were formulated for critically and noncritically phase-matched crystals.

It is beyond doubt that future scientific and commercial applications will require high peak and average power in a coherent optical field. This requires further 
investigations and development of suitable technologies. It is believed that the results presented in this work give a strong case for use of OPCPA as the near-term amplification technology of choice. 


\section{References}

1. P. A. Franken, A. E. Hill, C. W. Peters, and G. Weinrich, "Generation of optical harmonics," Phys. Rev. Lett. 7, 118-119 (1961).

2. C. C. Wang and G. W. Racette, "Measurement of parametric gain accompanying optical difference frequency generation," Appl. Phys. Lett. 6, 169-171 (1965).

3. J. A. Giordmaine and R. C. Miller, "Tunable coherent parametric oscillation in $\mathrm{LiNbO}_{3}$ at optical frequencies," Phys. Rev. Lett. 14, 973-976 (1965).

4. G. W. Baxter, Y. He, and B. J. Orr, "A pulsed optical parametric oscillator, based on periodically poled lithium niobate (PPLN), for high-resolution spectroscopy," Appl. Phys. B 67, 753-756 (1998).

5. W. G. Wagner and B. A. Lengyel, "Evolution of the giant pulse in a laser," J. Appl. Phys. 34, 2040-2046 (1963).

6. H. W. Mocker and R. J. Collins, "Mode competition and self-locking effects in a Q-switched ruby laser," Appl. Phys. Lett. 7, 270-273 (1965).

7. D. Strickland and G. Mourou, "Compression of amplified chirped optical pulses," Opt. Commun. 56, 219-221 (1985).

8. M. D. Perry, D. Pennington, B. C. Stuart, G. Tiethohl, J. A. Britten, C. Brown, S. Herman, B. Golick, M. Kartz, J. Miller, H. T. Powell, M. Vergino, and V. Yanovsky, "Petawatt laser pulses," Opt. Lett. 24, 160-162 (1999).

9. J. D. Bonlie, F. Patterson, D. Price, B. White, and P. Springer, "Production of $>10^{21} \mathrm{~W} / \mathrm{cm}^{2}$ from a large-aperture Ti:sapphire laser system," Appl. Phys. B 70, 155-160 (2000).

10. J. J. Macklin, J. D. Kmetec, and C. L. Gordon III, "High-Order Harmonic Generation Using Intense Femtosecond Pulses," Phys. Rev. Lett. 70, 766-769 (1993).

11. B. C. Stuart, P. S. Banks, M. D. Perry, M. D. Feit, R. S. Lee, F. Roeske, J. P. Armstrong, H. T. Nguyen, and J. A. Sefcik, "Femtosecond laser materials processing," Proc. SPIE 3269, 57-65 (1998). 
12. A. Dubietis, G. Jonasauskas, and A. Piskarskas, "Powerful femtosecond pulse generation by chirped and stretched pulse parametric amplification in BBO crystal," Opt. Commun. 88, 437-440 (1992).

13. I. N. Ross, P. Matousek, M. Towrie, A. J. Langley, and J. L. Collier, "The prospects for ultrashort pulse duration and ultrahigh intensity using optical parametric chirped pulse amplifiers," Opt. Commun. 144, 125-133 (1997).

14. W. R. Shen, "The Principles of Nonlinear Optics," John Wiley and Sons (1984).

15. R. W. Boyd, "Nonlinear Optics,” Academic Press (1992).

16. R. A. Baumgartner and R. L. Byer, “Optical Parametric Amplification," IEEE J. Quantum Electron. 15, 432-444 (1979).

17. J. A. Armstrong, N. Bloembergen, J. Ducuing, and P. S. Pershan, "Interactions between light waves in a nonlinear dielectric," Phys. Rev. 127, 1918-1939 (1962).

18. J. M. Manley and H. E. Rowe, "Some General Properties of Nonlinear Elements Part I. General Energy Relations," Proc. IRE 44, 904-913 (1956).

19. R. L. Sutherland, "Handbook of Nonlinear Optics," Marcel Dekker (1996).

20. J. A. Giordmaine, "Mixing of Light Beams in Crystals," Phys. Rev. Lett. 8, 19-20 (1962).

21. P. D. Maker, R. W. Terhune, M. Nisenhoff, and C. M. Savage, "Effect of Dispersion and Focusing on the Production of Optical Harmonics," Phys. Rev. Lett. 8, 21-22 (1962).

22. D. Eimerl, L. Davis, S. Velsko, E. K. Graham, A. Zalkin, "Optical, mechanical, and thermal properties of barium borate," J. Appl. Phys. 62, 1968-1983 (1987).

23. K. Kato, "Second-harmonic generation to $2048 \AA$ in $\beta-\mathrm{BaB}_{2} \mathrm{O}_{4}$," IEEE J. Quantum Electron. 22, 1013-1014 (1986).

24. N. P. Barnes, D. J. Gettemy, and R. S. Adhav, "Variation of the refractive index with temperature and the tuning rate for KDP isomorphs," J. Opt. Soc. Am. 72, 895-898 (1982).

25. R. C. Miller, G. D. Boyd, and A. Savage, "Nonlinear optical interactions in $\mathrm{LiNbO}_{3}$ without double refraction," Appl. Phys. Lett. 6, 77-79 (1965). 
26. D. N. Nikogosyan, "Lithium Triborate (LBO): a Review of Its Properties and Applications," Appl. Phys. A 58, 181-190 (1994).

27. E. J. Lim, M. M. Fejer, and R. L. Byer, "Second-harmonic generation of green light in periodically poled planar lithium niobate waveguide," Electron. Lett. 25, 174-175 (1989).

28. G. Imeshev, A. Galvanauskas, D. Harter, M. A. Arbore, M. Proctor, and M. M. Fejer, "Engineerable femtosecond pulse shaping by second-harmonic generation with Fourier synthetic quasi-phase-matching gratings," Opt. Lett. 23, 864-866 (1998).

29. A. Galvanauskas, D. Harter, M. A. Arbore, M. H. Chou, and M. M. Fejer, "Chirped-pulse-amplification circuits for fiber amplifiers, based on chirped-period quasi-phase-matching gratings," Opt. Lett. 23, 1695-1697 (1998).

30. M. J. Missey, V. Dominic, L. E. Myers, and R. C. Eckardt, "Diffusion-bonded stacks of periodically poled lithium niobate," Opt. Lett. 23, 664-666 (1998).

31. W. P. Risk, "Phase-matching characteristics of periodically-poled KTP," Proc. SPIE 2700, 78-84 (1996).

32. N. Bloembergen and P. S. Pershan, "Light Waves on a Boundary of Nonlinear Media," Phys. Rev. 128, 606-622 (1962).

33. M. Born and E. Wolf, "Principles of Optics," Cambridge University Press (1980).

34. E. S. Bliss, D. R. Speck, J. F. Holzrichter, J. H. Erkkila, and A. J. Glass, "Propagation of a high-intensity laser pulse with small-scale intensity modulation," Appl. Phys. Lett. 25, 448-450 (1974).

35. S. Backus, C. G. Durfee III, M. M. Murnane, and H. C. Kapteyn, "High power ultrafast lasers," Rev. Sci. Instrum. 69, 1207-1223 (1998).

36. G. Mourou, "The ultrahigh-peak-power laser: present and future," Appl. Phys. B 65, 205-211 (1997).

37. M. D. Perry and G. Mourou, "Terawatt to petawatt subpicosecond lasers," Science 264, 917-924 (1994).

38. E. B. Treacy, "Optical Pulse Compression With Diffraction Gratings, “ IEEE J. Quantum Electron. 5, 454-458 (1969). 
39. O. E. Martinez, "3000 times grating compressor with positive group velocity dispersion: application to fiber compensation in 1.3-1.6 $\mu \mathrm{m}$ region”, IEEE J. Quantum Electron. 23, 59-64 (1987).

40. O. E. Martinez, "Design of high-power ultrashort pulse amplifiers by expansion and recompression," IEEE J. Quantum Electron. 23, 1385-1387 (1987).

41. A. Sullivan, H. Hamster, H. C. Kapteyn, S. Gordon, W. White, H. Nathel, R. L. Blair, and R. W. Falcone, "Multiterawatt, 100-fs laser," Opt. Lett. 16, 1406-1408 (1991).

42. B. C. Stuart, M. D. Perry, J. Miller, G. Tietbohl, S. Herman, J. A. Britten, C. Brown, D. Pennington, V. Yanovsky, and K. Wharton, "125-TW Ti:sapphire/Nd:glass laser system," Opt. Lett. 22, 242-244 (1997).

43. R. D. Boyd, J. A. Britten, D. E. Decker, B. W. Shore, B. C. Stuart, M. D. Perry, and $\mathrm{L}$. $\mathrm{Li}$, "High-efficiency metallic diffraction gratings for laser applications," Appl. Opt. 34, 1697-1706 (1995).

44. D. M. Pennington, C. G. Brown, T. E. Cowan, S. P. Hatchett, E. Henry, S. Herman, M. Kartz, M. Key, J. Koch, A. J. MacKinnon, M. D. Perry, T. W. Phillips, M. Roth, T. C. Sangster, M. Singh, R. A. Snavely, M. Stoyer, B. C. Stuart, and S. C. Wilks, "Petawatt laser system and experiments," IEEE J. Sel. Top. Quantum Electron. 6, 676-688 (2000).

45. A. Piskarskas, D. Podenas, A. Stabinis, A. Umbrasas, A. Varanavichius, A. Yankauskas, and G. Yonushauskas, in "Ultrafast Phenomena V", SpringerVerlag, p 142 (1986).

46. I. N. Ross, P. Matousek, M. Towrie, A. J. Langley, J. L. Collier, C. N. Danson, C. Hernandez-Gomez, D. Neely, and K. Osvay, "Prospects for a multi-PW source using optical parametric chirped pulse amplifiers," Laser and Particle Beams 17, 331-340 (1999).

47. P. Matousek, B. Rus, and I. N. Ross, "Design of a Multi-Petawatt Optical Parametric Chirped Pulse Amplifier for the Iodine Laser ASTERIX IV," IEEE J. Quantum Electron. 36, 158-163 (2000).

48. H. Nakatani, W. R. Bosenberg, L. K. Cheng, and C. L. Tang, "Laser-induced damage in beta-barium metaborate," Appl. Phys. Lett. 53, $2587-2589$ (1988). 
49. A. E. Siegman, “Lasers,” University Science Books (1986).

50. P. Lacovara, L. Esterowitz, and M. Kokta, "Growth, spectroscopy, and lasing of titanium-doped sapphire," IEEE J. Quantum Electron. 21, 1614-1618 (1985).

51. M. D. Perry, T. Ditmire, and B. C. Stuart, "Self-phase modulation in chirpedpulse amplification," Opt. Lett. 19, 2149-2151 (1994).

52. A. Yariv, "Quantum Electronics," J. Wiley and Sons (1989).

53. W. H. Louisell, A. Yariv, and A. E. Siegman, "Quantum Fluctuations and Noise in Parametric Processes. I.," Phys. Rev. 124, 1646-1654 (1961).

54. D. A. Kleinman, “Theory of Optical Parametric Noise," Phys. Rev. 174, $1027-$ 1041 (1968).

55. B. C. Stuart, M. D. Feit, S. Herman, A. M. Rubenchik, B. W. Shore, and M. D. Perry, “Optical ablation by high-power short-pulse lasers," J. Opt. Soc. Am. B 13, 459-468 (1996).

56. D. Riedel, J. L. Hernandez-Pozos, R. E. Palmer, S. Baggott, K. W. Kolasinski, and J. S. Foord, "Tunable pulsed vacuum ultraviolet light source for surface science and materials spectroscopy based on high order harmonic generation," Rev. of Sci. Instrum. 72, 1977-1983 (2001).

57. M. Tabak, J. Hammer, M. E. Glinsky, W. L. Kruer, S. C. Wilks, J. Woodworth, E. M. Campbell, M. D. Perry, and R. J. Mason, "Ignition and high gain with ultrapowerful lasers," Phys. Plasmas 1, 1626-1634 (1994).

58. S. P. Velsko, W. F. Krupke, "Applications of high-average power nonlinear optics.," Proc. SPIE 2700, 6-17 (1996).

59. A. A. Isaev, M. A. Kazaryan, G. G. Petrash, S. G. Rautian, and A. M. Shalagin, "Evolution of Gaussian beams and pulse stimulated emission from lasers with unstable resonators,” Sov. J. Quantum Electron. 5, 607-614 (1975).

60. D. J. Armstrong, W. J. Alford, T. D. Raymond, and A. V. Smith, "Parametric amplification and oscillation with walkoff-compensating crystals," J. Opt. Soc. Am. B 14, 460-473 (1997).

61. J. Collier, C. Hernandez-Gomez, I. N. Ross, P. Matousek, C. N. Danson, J. Walczak, "Evaluation of an ultrabroadband high-gain amplification technique for chirped pulse amplification facilities," Appl. Opt. 38, 7486-7493 (1999). 
62. S. K. Zhang, M. Fujita, H. Yoshida, R. Kodama, H. Fujita, M. Nakatsuka, Y. Izawa, and C. Yamanaka, "Study on high gain broadband optical parametric chirped pulse amplification," Proc. SPIE 3886, 588-595 (2000).

63. S. K. Zhang, M. Fujita, M. Yamanaka, M. Nakatsuka, Y. Izawa, C. Yamanaka, "Study of the stability of optical parametric amplification," Opt. Commun. 184, 451-455 (2000).

64. P. S. Banks, "Frequency Conversion of High-Intensity, Femtosecond Laser Pulses," Ph. D. Thesis, University of California, Davis, UCRL-LR-128033 (1997).

65. I. N. Ross, J. L. Collier, P. Matousek, C. N. Danson, D. Neely, R. M. Allott, D. A. Pepler, C. Hernandez-Gomez, and K. Osvay, “Generation of terawatt pulses by use of optical parametric chirped pulse amplification," Appl. Opt. 39, 2422-2427 (2000).

66. G. Steinmeyer, D. H. Sutter, L. Gallman, N. Matuschek, and U. Keller, "Frontiers in Ultrashort Pulse Generation: Pushing the Limits in Linear and Nonlinear Optics," Science 286, 1507-1512 (1999).

67. T. Tajima and J. M. Dawson, "Laser Electron Accelerator," Phys. Rev. Lett. 43, 267-270 (1979).

68. T. E. Cowan, A. W. Hunt, T. W. Phillips, S. C. Wilks, M. D. Perry, C. Brown, W. Fountain, S. Hatchett, J. Johnson, M. H. Key, T. Parnell, D. M. Pennington, R. A. Snavely, and Y. Takahashi, "Photonuclear Fission from High Energy Electrons from Ultraintense Laser-Solid Interactions," Phys. Rev. Lett. 84, 903-906 (2000).

69. T. Ditmire, J. Zweiback, V. P. Yanovsky, T. E. Cowan, G. Hays, and K. B. Wharton, "Nuclear fusion from explosions of femtosecond laser-heated deuterium clusters," Nature 398, 489-492 (1999).

70. T. Guo, C. Spielmann, B. C. Walker, and C. P. J. Barty, "Generation of hard Xrays by ultrafast terawatt lasers," Rev. Sci. Instr. 72, 41-47 (2001).

71. R. Ell, U. Morgner, F. X. Kartner, J. G. Fujimoto, E. P. Ippen, V. Scheuer, G. Angelow, T. Tschudi, M. J. Lederer, A. Boiko, and B. Luther-Davies, "Generation of 5-fs pulses and octave-spanning spectra directly from a Ti:sapphire laser," Opt. Lett. 26, 373-375 (2001). 
72. K. Yamakawa, M. Aoyama, S. Matsuoka, H. Takuma, C. P. J. Barty, and D. Fittinghoff, "Generation of $16-\mathrm{fs}, 10-\mathrm{TW}$ pulses at a $10-\mathrm{Hz}$ repetition rate with efficient Ti:sapphire amplifiers," Opt. Lett. 23, 525-527 (1998).

73. S. A. Payne, L. K. Smith, L. D. DeLoach, W. L. Kway, J. B. Tassano, and W. F. Krupke, "Characterization of Cr:LiSAF solid-state laser properties," Proc. SPIE 1863, 24 (1993).

74. T. R. Boehly, Y. Fisher, D. D. Meyerhofer, W. Seka, J. M. Soures, and D. K. Bradley, "The effect of optical prepulse on direct-drive inertial confinement fusion target performance," Phys. Plasmas 8, 231-237 (2001).

75. N. P. Zaitseva, J. J. De Yoreo, M. R. Dehaven, R. L. Vital, K. E. Montgomery, M. Richardson, and L. J. Atherton, "Rapid growth of large-scale (40-55 cm) $\mathrm{KH}_{2} \mathrm{PO}_{4}$ crystals,” J. Crystal Growth 180, 255-262 (1997).

76. G. Cerullo, M. Nisoli, S. Stagira, and S. De Silvestri, "Sub-8-fs pulses from an ultrabroadband optical parametric amplifier in the visible," Opt. Lett. 23, 12831285 (1998).

77. A. Shirakawa, I. Sakane, M. Takasaka, and T. Kobayashi, "Sub-5-fs visible pulse generation by pulse-front-matched noncollinear optical parametric amplification," Appl. Phys. Lett. 74, 2268-2270 (1999).

78. P. Di Trapani, A. Andreoni, C. Solcia, P. Foggi, R. Danelius, A. Dubietis, and A. Piskarskas, "Matching of group velocities in three-wave parametric interaction with femtosecond pulses and application to traveling-wave generators," J. Opt. Soc. Am. B 12, 2237-2244 (1995).

79. A. Shirakawa and T. Kobayashi, "Noncollinearly phase-matched femtosecond optical parametric amplification with a $2000 \mathrm{~cm}^{-1}$ bandwidth," Appl. Phys. Lett. 72, 147-149 (1998).

80. A. Galvanauskas, A. Hariharan, D. Harter, M. A. Arbore, and M. M. Fejer, "High-energy femtosecond pulse amplification in a quasi-phase-matched parametric amplifier," Opt. Lett. 23, 210-212 (1998).

81. I. A. Begishev, A. A. Gulamov, E. A. Erofeev, E. A. Ibragimov, S. R. Kamalov, T. Usmanov, and A. D. Khadzhaev, "Highly efficient parametric amplification of 
optical beams. I. Optimization of the profiles of interacting waves in parametric amplification," Sov. J. Quantum Electron. 20, 1100-1103 (1990).

82. I. A. Begishev, A. A. Gulamov, E. A. Erofeev, E. A. Ibragimov, S. R. Kamalov, T. Usmanov, and A. D. Khadzhaev, "Highly efficient parametric amplification of optical beams. II. Parametric interaction of waves with conformal profiles," Sov. J. Quantum Electron. 20, 1104-1106 (1990).

83. B. A. Richman, S. E. Bisson, R. Trebino, E. Sidick, and A. Jacobson, "All-Prism Achromatic Phase Matching for Tunable Second-Harmonic Generation," Appl. Opt. 38, 3316-3323 (1999).

84. V. P. Yanovsky, T. Ditmire, G. Hays, T. Cowan, G. LeSage, and M. D. Perry, "Multiterawatt laser-linac facility," Conference on Lasers and Electro-Optics, Baltimore, MD, USA, 410 (1999).

85. P. S. Banks, M. D. Perry, V. Yanovsky, S. N. Fochs, B. C. Stuart, and J. Zweiback, "Novel all-reflective stretcher for chirped-pulse amplification of ultrashort pulses,” IEEE J. Quantum Electron. 36, 268-74 (2000).

86. B. C. Stuart, personal communication.

87. C. Toth, C. Barty, J. Squier, K. Yamakawa, K. R. Wilson, D. Fittinghoff, D.-E. Kim, C. Rose-Petruck, T. Guo, and B. Walker, "A 50 EW/cm² Ti:sapphire laser system for studying relativistic light-matter interactions," Opt. Exp. 5, 196-202 (1999).

88. W. Koechner, "Solid-State Laser Engineering," Springer-Verlag (1996).

89. D. Eimerl, "High Average Power Harmonic Generation," IEEE J. Quantum Electron. 23, 575-592 (1987).

90. C. A. Ebbers, "Frequency Conversion of High Peak and High Average Power Lasers," UCRL-JC-127266 (1997).

91. S. P. Velsko and W. F. Krupke, "Applications of high-average power nonlinear optics," Proc. SPIE 2700, 6-15 (1996).

92. “CASTECH's BBO Structural and Physical Properties", CASTECH product catalogue (2001).

93. Y. X. Fan, R. C. Eckardt, R. L. Byer, C. Chen, and A. D. Jiang, "Barium borate optical parametric oscillator,” IEEE J. Quantum Electron. 25, 1196-1199 (1989). 
94. V. G. Dmitriev, G. G. Gurzadyan, and D. N. Nikogosyan, "Handbook of Nonlinear Optical Crystals," Springer-Verlag (1999).

95. J. D. Beasley, "Thermal conductivities of some novel nonlinear optical materials," Appl. Opt. 33, 1000-1003 (1994).

96. C. A. Ebbers, personal communication.

97. M. A. Dreger, J. H. Erkkila, and D. Stone, "Coupled thermal and nonlinear effects for beam propagation in anisotropic crystals," Proc. SPIE 2145, 254-269 (1994).

98. B. J. Comaskey, personal communication.

99. Y. Hirano, Y. Koyata, S. Yamamoto, K. Kasahara, and T. Tajime, "208-W TEM 00 operation of a diode-pumped Nd:YAG rod laser," Opt. Lett. 24, 679-681 (1999).

100. Y. Hirano, N. Pavel, S. Yamamoto, Y. Koyata, and T. Tajime, "100-W class diode-pumped Nd:YAG MOPA system with a double-stage relay-optics scheme," Opt. Commun. 170, 275-280 (1999).

101. J. M. Auerbach and V. P. Karpenko, "Serrated-aperture apodizers for high-energy laser systems," Appl. Opt. 33, 3179-3183 (1994).

102. Cutting Edge Optronics, product catalogue.

103. Q. Lü, N. Kugler, H. Weber, S. Dong, N. Müller, and U. Wittrock, “A novel approach for compensation of birefringence in cylindrical Nd:YAG rods," Opt. and Quantum Electron. 28, 57-69 (1996).

104. W. C. Scott and M. de Witt, "Birefringence compensation and TEM 00 mode enhancement in a Nd:YAG laser," Appl. Phys. Lett. 18, 3-4 (1971).

105. G. D. Boyd and D. A. Kleinman, "Parametric interaction of focused Gaussian light beams," J. Appl. Phys. 39, 3597-3639 (1968).

106. S. K. Wong, G. Fournier, P. Mathieu, and P. Pace, "Beam divergence effects on nonlinear frequency mixing," J. Appl. Phys. 71, 1091-1101 (1992).

107. A. E. Siegman, "New developments in laser resonators," Proc. SPIE 1224, 2-14 (1990).

108. D. Eimerl, "Frequency conversion materials from a device perspective," Proc. SPIE 681, 2-59 (1987). 
109. J. D. Lindl, R. L. McCrory, and E. M. Campbell, "Progress Toward Ignition and Burn Propagation in Inertial Confinement Fusion," Phys. Today 45 (9) 32-40 (1992).

110. J. H. Nuckolls, L. Wood, A. Thiessen, and G. B. Zimmerman, "Laser compression of matter to super-high densities: thermonuclear (CTR) applications," Nature 239, 139-142 (1972).

111. J. D. Lindl, "Inertial Confinement Fusion: The Quest for Ignition and Energy Gain Using Indirect Drive," Springer-Verlag (1998).

112. J. T. Hunt and D. R. Speck, "Present and future performance of the Nova laser system", Opt. Engin. 28, 461-468 (1989).

113. T. R. Boehly, R. S. Craxton, T. H. Hinterman, P. A. Jaanimagi, J. H. Kelly, T. J. Kessler, R. L. Kremens, S. A. Kumpan, S. A. Letzring, R. L. McCrory, S. F. B. Morse, W. Seka, S. Skujsky, J. M. Soures, and C. P. Verdon, "The upgrade to the OMEGA laser system," Fusion Technology 26, 722-729 (1994).

114. W. J. Hogan, E. I. Moses, B. E. Warner, M. S. Sorem, and J. M. Soures, “The National Ignition Facility," Nuclear Fusion 41, 567-573 (2001).

115. M. Tabak, J. Hammer, M. E. Glinsky, W. L. Kruer, S. C. Wilks, J. Woodworth, E. M. Campbell, M. D. Perry, and R. J. Mason, "Ignition and high gain with ultrapowerful lasers," Phys. Plasmas 1, 1626-1634 (1994).

116. M. H. Key, M. D. Cable, T. E. Cowan, K. G. Estabrook, B. A. Hammel, S. P. Hatchett, E. A. Henry, D. E. Hinkel, J. D. Kilkenny, J. A. Koch, W. L. Kruer, A. B. Langdon, B. F. Lasinski, R. W. Lee, B. J. MacGowan, A. MacKinnon, J. D. Moody, M. J. Moran, A. A. Offenberger, D. M. Pennington, M. D. Perry, T. J. Phillips, T. C. Sangster, M. S. Singh, M. A. Stoyer, M. Tabak, G. L. Tietbohl, M. Tsukamoto, K. Wharton, and S. C. Wilks, "Hot electron production and heating by hot electrons in fast ignitor research," Phys. Plasmas 5, 1966-72 (1998).

117. R. Kodama, P. A. Norreys, K. Mima, A. E. Dangor, R. G. Evans, H. Fujita, Y. Kitagawa, K. Krushelnick, T. Miyakoshi, N. Miyanaga, T. Norimatsu, S. J. Rose, T. Shozaki, K. Shigemori, A. Sunahara, M. Tampo, K. A. Tanaka, Y. Toyama, T. Yamanaka, and M. Zepf, "Fast heating of ultrahigh-density plasma as a step towards laser fusion ignition," Nature 412, 798-802 (2001). 
118. M. H. Key, personal communication.

119. B. C. Stuart, S. Herman, and M. D. Perry, "Chirped-pulse amplification in Ti:sapphire beyond $1 \mu \mathrm{m}$," IEEE J. Quantum Electron. 31, 528-538 (1995).

120. M. D. Martinez, K. M. Skulina, F. J. Deadrick, J. K. Crane, B. Moran, J. Braucht, B. Jones, S. Hawkins, R. Tilley, J. Crawford, D. Browning, and F. Penko, "Performance results of the high gain, $\mathrm{Nd}$ :glass, engineering prototype preamplifier module (PAM) for the National Ignition Facility (NIF)," Proc. SPIE 3611, 169-180 (1999).

121. M. D. Perry, R. D. Boyd, J. A. Britten, D. Decker, B. W. Shore, C. Shannon, and E. Shults, "High-efficiency multilayer dielectric diffraction gratings," Opt. Lett. 20, 940-942 (1995).

122. T. Ditmire, J. Zweiback, V. P. Yanovsky, T. E. Cowan, G. Hays, and K. B. Wharton, "Nuclear fusion from explosions of femtosecond laser-heated deuterium clusters," Nature 398, 489-492 (1999).

123. D. L. Burke, R. C. Field, G. Horton-Smith, J. E. Spencer, D. Walz, S. C. Berridge, W. M. Bugg, K. Shmakov, A. W. Weidemann, C. Bula, K. T. McDonald, E. J. Prebys, C. Bamber, S. J. Boege, T. Koffas, T. Kotseroglou, A. C. Melissinos, D. D. Meyerhofer, D. A. Reis, and W. Ragg, "Positron Production in Multiphoton Light-by-Light Scattering," Phys. Rev. Lett. 79, 1626-1629 (1997).

124. D. D. Meyerhofer, "High-Intensity-Laser-Electron Scattering," IEEE J. Quantum Electron. 33, 1935-1941 (1997).

125. T. Tajima and J. M. Dawson, "Laser Electron Accelerator," Phys. Rev. Lett. 43, 267-270 (1979).

126. T. E. Cowan, A. W. Hunt, T. W. Phillips, S. C. Wilks, M. D. Perry, C. Brown, W. Fountain, S. Hatchett, J. Johnson, M. H. Key, T. Parnell, D. M. Pennington, R. A. Snavely, and Y. Takahashi, "Photonuclear Fission from High Energy Electrons from Ultraintense Laser-Solid Interactions," Phys. Rev. Lett. 84, 903-906 (2000).

127. M. D. Perry and B. W. Shore, "Petawatt Laser Report," UCRL-ID-124933, 1-9 (1996).

128. B. A. Remington, R. P. Drake, H. Takabe, and D. Arnett, "A review of astrophysics experiments on intense lasers," Phys. Plasmas 7, 1641-1652 (2000). 
129. S. Shibu, J. Parashar, and H. D. Pandey, "Possibility of pulse compression of a short-pulse laser in a plasma," J. Plasma Phys. 59, 91-96 (1998).

130. V. M. Malkin, G. Shvets, and N. J. Fisch, "Fast Compression of Laser Beams to Highly Overcritical Powers," Phys. Rev. Lett. 82,4448-4451 (1999).

131. A. M. Rubenchik, personal communication.

132. C. P. J. Barty, G. Korn, F. Raksi, C. Rose-Petruck, J. Squier, A.-C. Tien, K. R. Wilson, V. V. Yakovlev, and K. Yamakawa, "Regenerative pulse shaping and amplification of ultrabroadband optical pulses," Opt. Lett. 21, 219-221 (1996).

133. I. N. Ross, P. Matousek, M. Towrie, A. J. Langley, J. L. Collier, C. N. Danson, C. Hernandez-Gomez, D. Neely, and K. Osvay, "Prospects for a multi-PW source using optical parametric chirped pulse amplifiers," Laser and Particle Beams 17, 331-340 (1999).

134. M. D. Perry, B. C. Stuart, P. S. Banks, M. D. Feit, V. Yanovsky, and A. M. Rubenchik, "Ultrashort-pulse laser machining of dielectric materials," J. Appl. Phys. 85, 6803-6810 (1999).

135. B. C. Stuart, M. D. Feit, A. M. Rubenchik, B. W. Shore, and M. D. Perry, "Laserinduced damage in dielectrics with nanosecond to subpicosecond pulses," Phys. Rev. Lett. 74, 2248-2251 (1995).

136. B. C. Stuart, M. D. Feit, S. Herman, A. M. Rubenchik, B. W. Shore, and M. D. Perry, "Nanosecond-to-femtosecond laser-induced breakdown in dielectrics," Phys. Rev. B 53, 1749-1761 (1996).

137. J. P. Armstrong, P. S. Banks, M. D. Feit, R. S. Lee, M. D. Perry, F. Roeske, and B. C. Stuart, "Laser Cutting of Pressed Explosives," UCRL-JC-128373 (1998).

138. R. J. Beach, E. C. Honea, S. B. Sutton, C. M. Bibeau, J. A. Skidmore, M. A. Emanuel, S. A. Payne, P. V. Avizonis, R. S. Monroe, and D. G. Harris, "Highaverage-power diode-pumped Yb:YAG lasers," Proc. SPIE 3889, 246-260 (2000).

139. F. Salin, C. Le Blanc, J. Squier, and C. Barty, "Thermal eigenmode amplifiers for diffraction-limited amplification of ultrashort pulses," Opt. Lett. 23, 718-720 (1998).

140. J. Stone, "CW Raman fiber amplifier," Appl. Phys. Lett. 26, 163-165 (1975). 
141. N. Edagawa, K. Mochizuki, and H. Wakabayashi, "1.2 Gbit/s, 218 km transmission experiment using inline Er-doped optical fibre amplifier," Electron. Lett. 25, 363-365 (1989). 